\title{
Vom Wohlergehen der Nationen
}

\section{DIE ROLLE VON HUMAN- UND SOZIALKAPITAL}

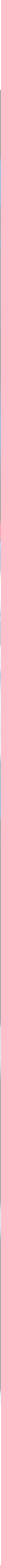




\section{Vom Wohlergehen der Nationen}

\section{DIE ROLLE VON HUMAN- UND SOZIALKAPITAL}

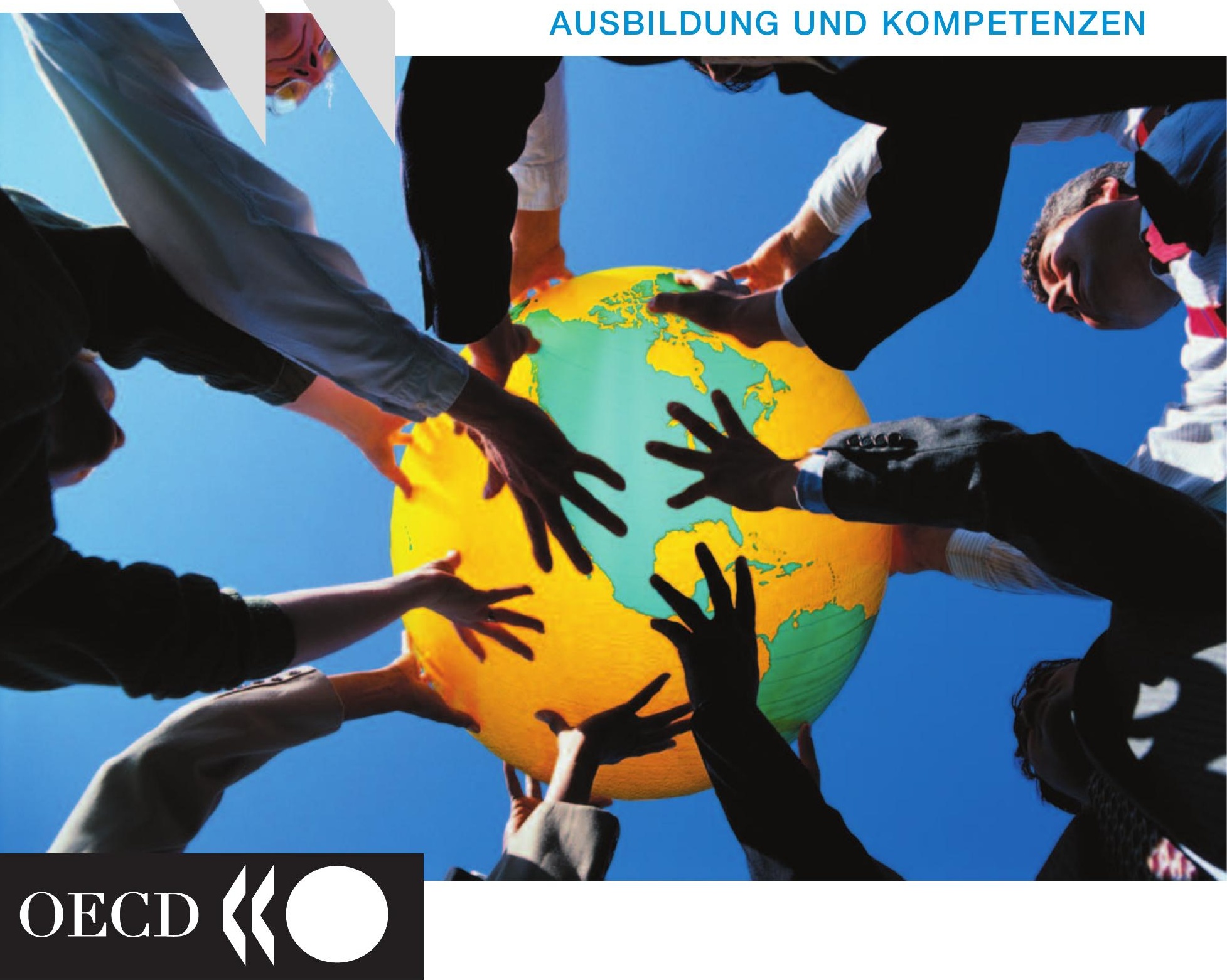



Zentrum für Forschung und Innovation im Bildungswesen

\section{Vom Wohlergehen der Nationen}

\section{DIE ROLLE VON HUMAN- UND SOZIALKAPITAL}

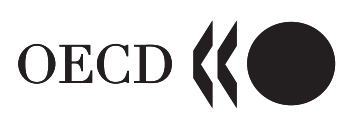




\section{ORGANISATION FÜR WIRTSCHAFTLICHE ZUSAMMENARBEIT UND ENTWICKLUNG}

Gemäß Artikel 1 des am 14. Dezember 1960 in Paris unterzeichneten und am 30. September 1961 in Kraft getretenen Übereinkommens fördert die Organisation für wirtschaftliche Zusammenarbeit und Entwicklung (OECD) eine Politik, die darauf gerichtet ist:

- in den Mitgliedstaaten unter Wahrung der finanziellen Stabilität eine optimale Wirtschaftsentwicklung und Beschäftigung sowie einen steigenden Lebensstandard zu erreichen und dadurch zur Entwicklung der Weltwirtschaft beizutragen;

- in den Mitglied- und Nichtmitgliedstaaten, die in wirtschaftlicher Entwicklung begriffen sind, zu einem gesunden wirtschaftlichen Wachstum beizutragen; und

- im Einklang mit internationalen Verpflichtungen auf multilateraler und nicht diskriminierender Grundlage zur Ausweitung des Welthandels beizutragen.

Die Gründungsmitglieder der OECD sind: Belgien, Dänemark, Deutschland, Frankreich, Griechenland, Irland, Island, Italien, Kanada, Luxemburg, Niederlande, Norwegen, Österreich, Portugal, Schweden, Schweiz, Spanien, Türkei, Vereinigtes Königreich und Vereinigte Staaten. Folgende Staaten wurden zu den nachstehend genannten Daten Mitglieder der OECD: Japan (28. April 1964), Finnland (28. Januar 1969), Australien (7. Juni 1971), Neuseeland (29. Mai 1973), Mexiko (18. Mai 1994), die Tschechische Republik (21. Dezember 1995), Ungarn (7. Mai 1996), Polen (22. November 1996), Korea (12. Dezember 1996) und die Slowakische Republik (14. Dezember 2000). Die Kommission der Europäischen Gemeinschaften nimmt an den Tätigkeiten der OECD teil (Artikel 13 des Übereinkommens über die OECD).

Das Zentrum für Forschung und Innovation im Bildungswesen (CERI) wurde im Juni 1968 vom Rat der Organisation für wirtschaftliche Zusammenarbeit und Entwicklung ins Leben gerufen. Alle OECD-Mitgliedsländer nehmen daran teil.

Die Hauptziele dieses Zentrums sind:

- Förderung und Unterstützung der Entwicklung von Forschungsaktivitäten im Bildungsbereich und gegebenenfalls die Durchführung solcher Forschungsaktivitäten;

- Förderung und Unterstützung von Pilotversuchen im Hinblick auf die Einführung und Erprobung von Innovationen im Bildungssystem;

- Förderung der Entwicklung einer Zusammenarbeit zwischen den Mitgliedstaaten auf dem Gebiet der pädagogischen Forschung und Innovation.

Das Zentrum arbeitet innerhalb der Organisation für wirtschaftliche Zusammenarbeit und Entwicklung gemäß den Entscheidungen des Rats der Organisation und im Auftrag des Generalsekretärs sowie unter der direkten Kontrolle eines Lenkungsausschusses, in den jeder am Arbeitsprogramm des CERI beteiligte Mitgliedstaat einen nationalen Experten für den Aufgabenbereich des Zentrums entsendet.

Originalfassungen veröffentlicht unter dem Titel:

The Well-being of Nations: The Role of Human and Social Capital

Du bien-être des nations : Le rôle du capital humain et social

(c) OECD 2001

(c) OECD 2004 für die deutsche Fassung

Genehmigungen zum Nachdruck von Teilen dieses Werks für nichtkommerzielle Zwecke oder zur Verwendung im Unterricht sind einzuholen beim Centre français d'exploitation du droit de copie (CFC), 20, rue des Grands-Augustins, 76006 Paris, Frankreich, tel: (33-1) 44 07 47 70, fax: (33-1) 463467 19. Dies gilt für alle Länder mit Ausnahme der Vereinigten Staaten, wo das Copyright Clearance Center Inc. (CCC), Customer Service, tel: (508) 750-8400, 222 Rosewood Drive, Danvers, MA 01923, USA oder CCC online: www.copyright.com die entsprechenden Genehmigungen erteilt. Alle sonstigen Anträge auf Überlassung von Nachdruck- oder Übersetzungsrechten für das gesamte Dokument oder Teile davon sind zu richten an: OECD Publications, 2, rue André-Pascal, 75775 Paris Cedex 16, Frankreich. 


\section{VORWORT}

Der vorliegende Bericht ist eine Veröffentlichung des OECD-Zentrums für Forschung und Innovation im Bildungswesen. Das Autorenteam Tom Healy und Sylvain Côté konnte sich auf wertvolle Beiträge von John F. Helliwell (University of British Columbia, Kanada), Simon Field (CERI/OECD) wie auch vielen anderen Kollegen des OECD-Sekretariats stützen.

Besonders nützlich waren ferner die von zahlreichen Hochschulexperten und Ländervertretern auf OECD-Ausschusssitzungen formulierten Ratschläge und Empfehlungen, die als Orientierungshilfe für die Arbeiten dienten. Von besonderer Bedeutung waren darüber hinaus zwei externe Veranstaltungen: Zum einen ein internationales Symposium zum Thema The Contribution of Human and Social Capital to Sustained Economic Growth and Well-being (Der Beitrag von Human- und Sozialkapital zu nachhaltigem Wirtschaftswachstum und Wohlergehen), das von Human Resources Development Canada (HRDC) und der OECD im März 2000 in Quebec-Stadt, Kanada, gemeinsam organisiert und getragen wurde, und zum anderen die Tagung einer Expertengruppe für Human- und Sozialkapital bei der OECD im Juli 2000, wo der erste Entwurf des vorliegenden Berichts geprüft wurde.

Für diesen Bericht zeichnet der Generalsekretär der OECD verantwortlich. 


\section{DANK}

Unter den internationalen Fachleuten, die an der Erstellung des Berichts beteiligt waren, möchten wir folgenden Personen ganz besonders danken: Gunnar Eliasson (Royal Institute of Technology, Stockholm), Dominique Foray (Université Dauphine, Paris), David Halpern (University of Cambridge, Vereinigtes Königreich), Tom Kellaghan (Education Research Centre, Irland), Bengt-Aake Lundvall (Universität Aalborg, Dänemark), Peter MacDonagh (Berater des irischen Premierministers), Lars Osberg (University of Dalhousie, Kanada), Robert D. Putnam (Harvard University, Vereinigte Staaten), Jo Ritzen (Vizepräsident, Weltbank), Tom Schuller (Birkbeck College, Vereinigtes Königreich), Simon Szreter (University of Cambridge, Vereinigtes Königreich), Jonathan Temple (Oxford College, Vereinigtes Königreich), Doug Willms (University of New Brunswick, Kanada), Michael Woolcock (Weltbank), Jean-Pierre Worms (Centre de Sociologie des Organisations, Paris).

Besonderer Dank gebührt Human Resources Development Canada (HRDC), namentlich Jean-Pierre Voyer und Richard Roy, für die Unterstützung des Projekts in seinen verschiedenen Stadien, die Finanzierung der Entsendung von Sylvain Côté zum OECD-Sekretariat sowie die Mitträgerschaft des Symposiums in Quebec-Stadt im März 2000.

(C) OECD 2004 


\section{INHALTSVERZEICHNIS}

Einleitung

Kapitel 1 Neu aufkommende soziale und wirtschaftliche Probleme

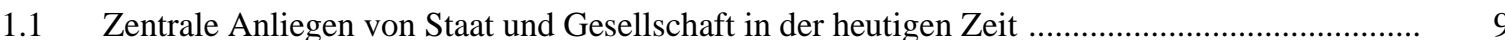

1.2 Die derzeitige Entwicklung in einigen dieser Schlüsselbereiche ............................................. 10

1.3 Die Wechselbeziehungen zwischen Wohlergehen auf der einen und Human- und Sozialkapital auf der anderen Seite ................................................................... 13

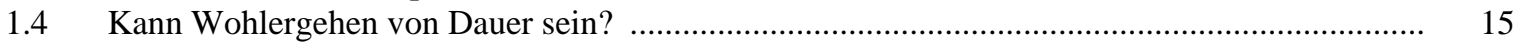

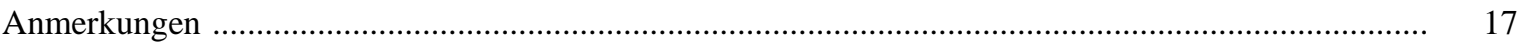

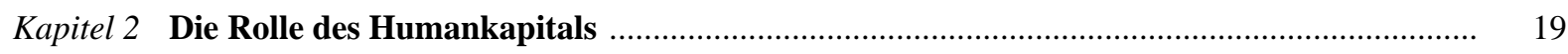

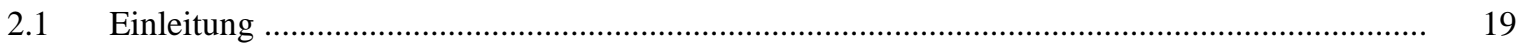

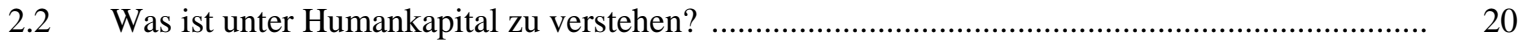

2.3 Wie kann Humankapital gemessen werden? ........................................................................ 23

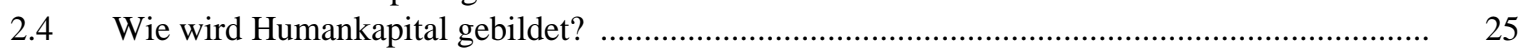

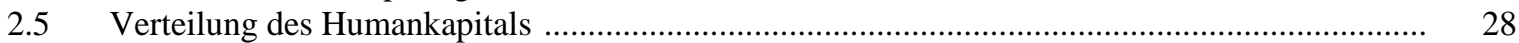

2.6 Entwicklung der Nachfrage nach Humankapital …......................................................... 31

2.7 Welchen Einfluss hat das Humankapital auf das wirtschaftliche Wohlergehen? ..................... 33

2.8 Welchen Einfluss hat das Humankapital auf die verschiedenen Aspekte des

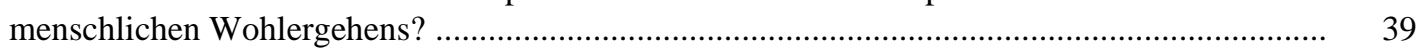

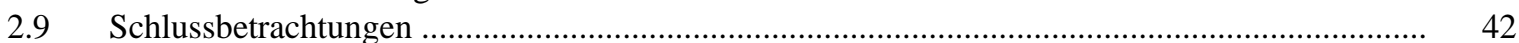

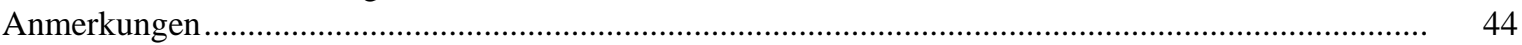

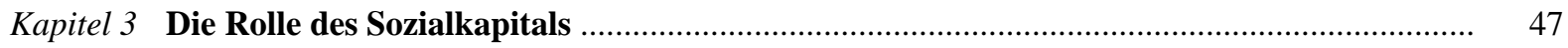

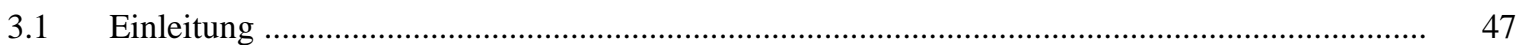

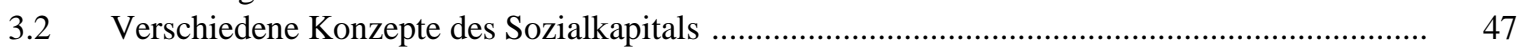

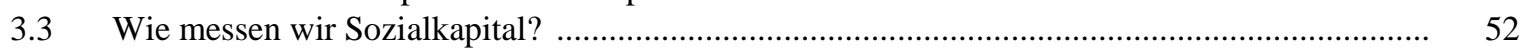

3.4 Die Quellen des Sozialkapitals? ................................................................................ 55

3.5 Unterliegen Vertrauen und bürgergesellschaftliches Engagement im Laufe der Zeit

3.6 Worauf sind die Veränderungen beim Vertrauensniveau und beim bürgergesellschaftlichen Engagement zurückzuführen?

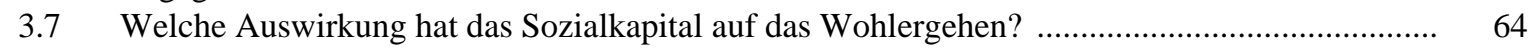

3.8 Relation zwischen Sozialkapital und sozialer Ungleichheit .............................................. 69

3.9 Auswirkungen des Sozialkapitals auf den wirtschaftlichen Wohlstand .................................. 71

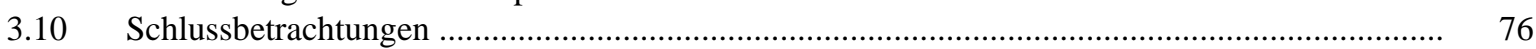

Anmerkungen

Kapitel 4 Konsequenzen für die staatliche Politik und weiterer Forschungsbedarf ......................... 79

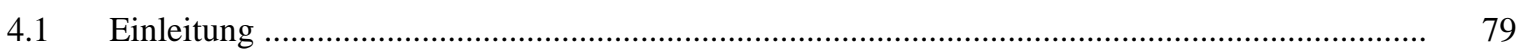

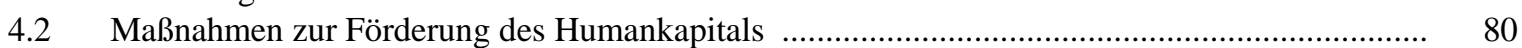

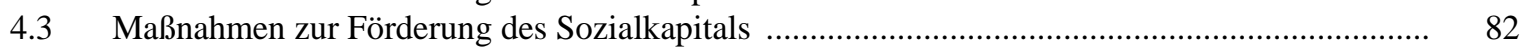

4.4 Wissensdefizite und künftige Forschungsbereiche ....................................................... 85

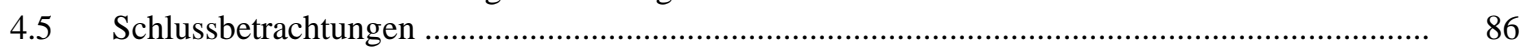

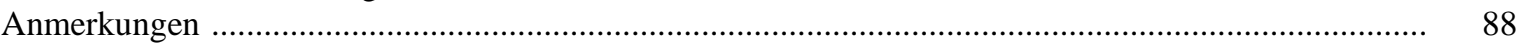


Anhang A Verschiedene Messgrößen für das Wohlergehen

Anhang $B$ Wichtige Trends bei den sozialen und ökonomischen Rahmenbedingungen

Anhang $C$ Bestimmungsfaktoren für das Bildungsniveau: Forschungsergebnisse

Anhang $D$ Auswirkungen des Humankapitals auf das Wirtschaftswachstum: einige wichtige Ergebnisse

Anhang $E$ Nehmen Vertrauen und bürgergesellschaftliches Engagement in den OECD-Ländern ab? ....

Literaturverzeichnis

Tabellen

3.1 Eine Messgröße des Vertrauens (World Values Study), 1995-1996

D.1 Regressionsanalysen zu den Auswirkungen der Bildung im Ländervergleich:

einige wichtige Untersuchungen.

Abbildungen

1.1 Beziehungen zwischen menschlichem Wohlergehen, wirtschaftlichem Wohlstand und BIP

1.2 Wichtigste Inputs für menschliches Wohlergehen und ihre Wechselbeziehungen

2.1 Komparative Verteilung des Grundbildungsniveaus

A.1 Veranschaulichung des Osberg-Indexes

A.2 Trends des wirtschaftlichen Wohlergehens im internationalen Vergleich

B.1 Reales Pro-Kopf-BIP, in konstanten Preisen, Durchschnitt auf der Basis ausgewählter OECD-Länder, 1966-1999

Entwicklung der Einkommensungleichheit, für die gesamte Bevölkerung, in ausgewählten

B.2 EntwicD-Ländern, Mitte der achtziger und neunziger Jahre

B.3 Prozentualer Anteil der in relativer Armut lebenden Kinder, in ausgewählten OECD-Ländern, neunziger Jahre

B.4 Arbeitslosenquoten der 25- bis 54-Jährigen und der 15- bis 24-Jährigen, in ausgewählten OECD-Ländern, 1975-1999

B.5 Altenquotienten in den OECD-Ländern, 1950-2050

B.6 Prozentualer Anteil der Bevölkerung der OECD-Länder im Alter von 25-64 Jahren mit Sekundar- oder Hochschulniveau, 1950-1998

B.7 Inzidenzrate der Alleinerziehenden in ausgewählten OECD-Ländern, Vergleich zwischen den achtziger und neunziger Jahren

B.8 Unterschiede bei den Arbeitsverdiensten zwischen Männern und Frauen,
25- bis 64- jährige Vollzeiterwerbstätige, achtziger und neunziger Jahre

B.8 Unterschiede bei den Arbeitsverdiensten zwischen Männern und Frauen,
25- bis 64- jährige Vollzeiterwerbstätige, achtziger und neunziger Jahre

B.9 Durchschnittliche Erwerbsbeteiligung der 15- bis 64-jährigen Männer und Frauen, in ausgewählten OECD-Ländern, 1960-1999

B.10 Zugänge von Ausländern in ausgewählten OECD-Ländern, 1999

B.14 Jüngste Tendenzen in Bezug auf die Lebenszufriedenheit, 12 ausgewählte Länder der Europäischen Union, 1973, 1983 und 1997

B.15 Prozentsatz der jeweiligen Personen, die die Frage nach ihrem subjektiven Glücksempfinden unterschiedlich beantworten, Vereinigte Staaten, 1972-1998

B.16 Suizidquote in ausgewählten OECD-Ländern, 1950-1997

B.17 Treibhausgasemissionen in den OECD-Ländern, gemäß unterschiedlichen Szenarien, 1990-2010

E.1 Schwund der Teilnehmer an Vereinsversammlungen in den Vereinigten Staaten, 1975-1999

E.2 Gestiegene Nachfrage nach juristischem und Sicherheitspersonal in den Vereinigten Staaten, 1900-1997 


\section{EINLEITUNG}

Über die Rolle des Humankapitals bei der Förderung der wirtschaftlichen und sozialen Entwicklung wird seit langem diskutiert, doch ist nach wie vor umstritten, wie groß diese Rolle wirklich ist. In den vergangenen Jahren hat auch das Interesse an der Bedeutung des Sozialkapitals zugenommen, um der Frage auf den Grund zu gehen, ob und inwieweit soziale Beziehungen wie auch ganz individuelle Eigenschaften einen wesentlichen Beitrag zu Wirtschaftstätigkeit und menschlichem Wohlergehen leisten.

Dieser Bericht dient einem dreifachen Zweck: a) Beschreibung der neuesten Untersuchungsergebnisse zur Frage der Humankapitalinvestitionen und ihres Einflusses auf Wachstum und Wohlergehen; $b$ ) Beschreibung und Präzisierung des noch neueren Konzepts des Sozialkapitals sowie $c$ ) Ermittlung der jeweiligen Rolle von Human- und Sozialkapital bei der Verwirklichung einer nachhaltigen wirtschaftlichen und sozialen Entwicklung. Dieser Bericht stellt einen Beitrag zu den OECD-Projekten über Wirtschaftswachstum und nachhaltige Entwicklung dar. Ferner schließt er an den OECD-Bericht Human Capital Investment - An International Comparison an, der 1998 veröffentlicht wurde.

Kapitel 1 umreißt die wichtigsten im Bericht behandelten Anliegen, Trends, Konzepte und Zusammenhänge wie auch die großen Fragen, die sich in Bezug auf diese Problematik stellen. Kapitel 2 ist der Definition und Messung des Humankapitals gewidmet, wobei alle Arten von Kompetenzen und individuellen Eigenschaften untersucht und berücksichtigt werden, die für das Wohlergehen von Bedeutung sind. Dabei werden die Quellen des Humankapitals in Familie und Schule, in gesellschaftlichen Gruppen und Gemeinwesen sowie am Arbeitsplatz erörtert, unter besonderer Berücksichtigung der Verteilung der Lernmöglichkeiten und -ergebnisse zwischen verschiedenen Gruppen. Ferner werden in diesem Kapitel die empirischen Daten über den Einfluss des Humankapitals auf Wirtschaft und Gesellschaft bewertet. Kapitel 3 befasst sich mit Definition und Messung des Sozialkapitals, indem sowohl seine Quellen als auch seine Auswirkungen auf ein breites Spektrum möglicher Ergebnisse untersucht werden. Erörtert werden ferner die potentiellen Komplementaritäten von Human- und Sozialkapital, und zwar sowohl vom konzeptuellen wie auch vom empirischen Standpunkt aus. In Kapitel 4 werden einige grundlegende Fragen hinsichtlich der Tragweite der vorangegangenen Human- und Sozialkapitalanalysen für die staatliche Politik untersucht und der künftige Forschungs- und Datenbedarf auf internationaler Ebene ermittelt. Wesentlich in diesem Zusammenhang ist, dass sich zahlreiche Akteure an der Förderung hoch effizienter Investitionen in Personen, Organisationen, gesellschaftliche Gruppen und Gemeinwesen beteiligen. 



\section{NEU AUFKOMMENDE SOZIALE UND WIRTSCHAFTLICHE PROBLEME}

„Die Unterschiede zwischen Quantität und Qualität des Wachstums, seinen Kosten und seiner Rendite wie auch der kurz- und langfristigen Perspektive dürfen nicht aus den Augen verloren werden ... Lautet das Ziel „mehr“ Wachstum, dann sollte genauer definiert werden, wovon mehr Wachstum und zu welchem Zweck. "Simon Kuznets in The New Republic (1962).

Im vorliegenden Bericht werden Human- und Sozialkapital nicht als Selbstzweck, sondern in ihrer Eigenschaft als Ressourcen untersucht, die zur Förderung der wirtschaftlichen und sozialen Entwicklung eingesetzt werden können. Mit dem vorliegenden Kapitel soll die Debatte über Human- und Sozialkapital in den größeren Zusammenhang dieser globalen gesellschaftlichen Ziele gestellt werden.

\subsection{Zentrale Anliegen von Staat und Gesellschaft in der heutigen Zeit}

Soziale Integration und Lebensqualität zählen zu den wichtigsten Anliegen von Staat und Gesellschaft ...

... und Werte und Einstellungen verändern sich radikal.
Staat und Gesellschaft streben Wirtschaftswachstum an, setzen sich gleichzeitig aber auch zunehmend mit dessen Folgen für das natürliche und soziale Umfeld auseinander. Ihr Augenmerk gilt insbesondere der Ungleichheit, dem Potential für die Entstehung neuer Formen der Ausgrenzung und Armut unter dem Einfluss des wachsenden Technologieeinsatzes sowie generell der Lebensqualität und dem Gesundheitszustand von Kindern, alten Menschen sowie einzelnen Bürgern und Gruppen, die wirtschaftlich und sozial benachteiligt sind. Die Bewältigung dieser Anliegen wird angesichts des Wandels, der sich derzeit in den Arbeitsstrukturen, der Gestaltung des Familienlebens und beim Engagement im Gemeinwesen vollzieht, zu einer immer komplexeren Aufgabe.

Zurückzuführen sind diese neuen Probleme auf den grundlegenden Wandel der Wertvorstellungen und Strukturen des gesellschaftlichen Engagements, der sich derzeit in vielen OECD-Ländern vollzieht, und diese Neuorientierungen könnten eine Überprüfung der Politikziele erforderlich machen. Viele dieser Veränderungen gehen in Richtung einer größeren Diversität und einer stärkeren Betonung der Selbstverwirklichung des Einzelnen, der persönlichen Autonomie und Verantwortung, des subjektiven Wohlergehens und der Lebensqualität. Aus der World Values Study geht hervor, dass sich das subjektive Wohlbefinden ab einem bestimmten Pro-Kopf-Einkommen bei steigendem Einkommen weniger rasch erhöht (Inglehart, 1997). 
Wirtschaftliche und soziale Fortschritte müssen nicht unbedingt im Widerspruch zueinander stehen ...

... doch sollten wir uns mit der langfristigen Entwicklung des menschlichen Wohlergehens wie auch der kurzfristigen Erhöhung der wirtschaftlichen Produktion auseinander setzen.

Das rasche Wirtschaftswachstum hat zwar für einen Rückgang der absoluten Armut gesorgt ...

... doch bedeutet Wohlergehen mehr als wirtschaftlicher Wohlstand ...
Viele Beobachter haben die Reibungspunkte zwischen wirtschaftlichem Fortschritt und gesellschaftlichen Funktionsstörungen untersucht - beispielsweise die Auswirkungen des raschen technologischen Wandels, der Obsoleszenz von Qualifikationen, von Arbeitsplatzunsicherheit und längeren Arbeitszeiten auf die Arbeitskräfte. Der vorliegende Bericht liefert keinen Nachweis dafür, dass der Anstieg des wirtschaftlichen Wohlstands notwendigerweise zu Lasten der Sozialkapitalreserven gehen musste, legt aber dennoch den Schluss nahe, dass zwischen gewissen Aspekten des wirtschaftlichen Fortschritts und der Stresszunahme bzw. der Lockerung der sozialen Bindungen ein Zusammenhang besteht. Gleichzeitig eröffnen die neuen Produktions- und Arbeitsbedingungen aber auch ganz neue Möglichkeiten, die, sofern sie genutzt werden, zu einer deutlichen Verbesserung des Wohlergehens beitragen könnten.

Die sozialen Ziele sind umfassender als jene, die auf eine kurzfristige Erhöhung der wirtschaftlichen Produktion ausgerichtet sind, da a) die Steigerung des globalen und nicht nur des wirtschaftlichen Wohlergehens von Bedeutung ist und $b$ ) die langfristigen Auswirkungen ökonomischer, ökologischer und sozialer Trendentwicklungen in jeder Analyse der derzeit zur Verfügung stehenden Politikoptionen berücksichtigt werden müssen. Der Faktor Zeit spielt bei der Beurteilung der gegenwärtigen Investitions- und Produktionsstrukturen wie auch der antizipierten künftigen DownstreamEffekte eine entscheidende Rolle, da viele politische Entscheidungsprozesse im öffentlichen und privaten Sektor durch Ungewissheiten und lange Reifezeiten gekennzeichnet sind. Um nachhaltig für mehr Wohlergehen zu sorgen, kommt es entscheidend darauf an, die Folgen von Entwicklungen im menschlichen und sozialen wie auch im materiellen und natürlichen Umfeld zu verstehen.

\subsection{Die derzeitige Entwicklung in einigen dieser Schlüsselbereiche}

Seit einigen Jahrzehnten verzeichnen die OECD-Länder deutliche Zuwächse der gesamtwirtschaftlichen Produktion, die mit einem Anstieg des Lebensstandards und einer Verbesserung der Arbeitsbedingungen wie auch des Gesundheitszustands und des Bildungsniveaus einhergehen. Obwohl der steigende materielle Wohlstand nicht allen in gleichem Maße zugute gekommen ist, hat der Umfang der absoluten und extremen Armut in den OECD-Ländern doch seit den fünfziger Jahren abgenommen. Auch wenn Wirtschaftswachstum nicht das einzige Politikziel darstellt, so schafft es dennoch die finanziellen Voraussetzungen für die Überwindung von sozialer Ausgrenzung, Armut und gesundheitlichen Beeinträchtigungen. Nach einer beispiellosen Ausweitung der gesamtwirtschaftlichen Produktion stehen nun eher die „Qualität“ des Wirtschaftswachstums und die Frage, wie das Wohlergehen weiter gesteigert werden kann, im Mittelpunkt des Interesses ${ }^{1}$.

In Abbildung 1.1 werden drei Ebenen des Wohlergehens unterschie$\mathrm{den}^{2}$. Zum Wohlergehen gehört natürlich wirtschaftlicher Wohlstand, gleichzeitig aber auch der Besitz der Bürgerrechte, eine relative Freiheit von Kriminalität, eine saubere Umwelt sowie eine gute seelische und körperliche Verfassung. In diesem Sinne hebt Sen (1987) besonders die Bedeutung der Möglichkeiten bzw. „sozialen Fähigkeiten“ des einzelnen Bürgers hervor, seine Lebensziele nach eigenen Vorstellungen zu wählen und zu erreichen. 
Abbildung 1.1 Beziehungen zwischen menschlichem Wohlergehen, wirtschaftlichem Wohlstand und BIP

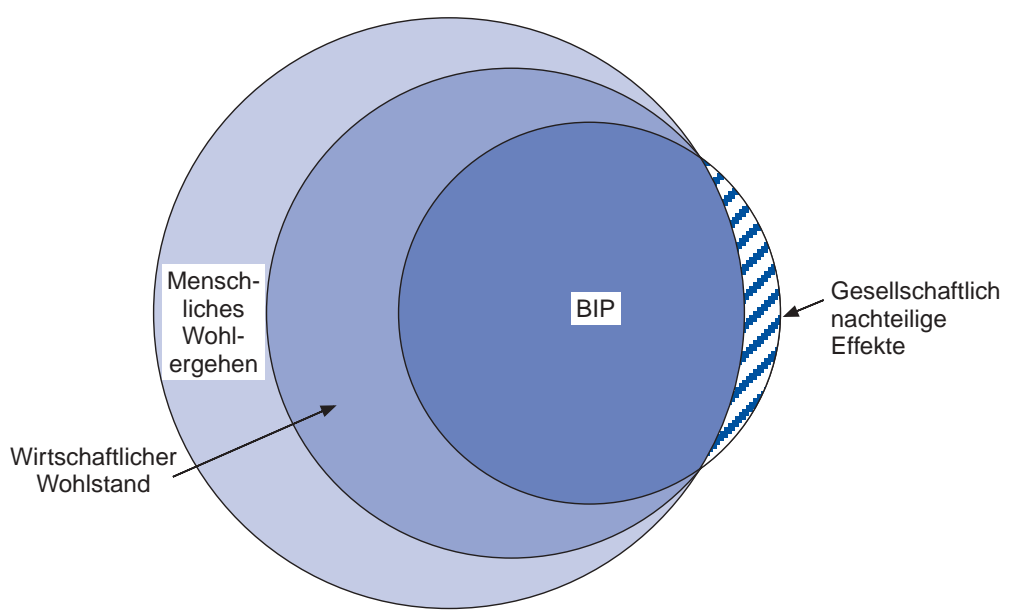

Quelle: OECD.

... und wirtschaftlicher Wohlstand geht über Messgrößen wie das BIP hinaus.
So gesehen ist das Wachstum der gesamtwirtschaftlichen Produktion kein Selbstzweck, sondern eine Voraussetzung für die Erweiterung der Wahlmöglichkeiten des Menschen (Arbeit, Freizeit oder politische und kulturelle Aktivitäten). Die erzielten Ergebnisse (in der Terminologie von Sen „Funktionsfähigkeiten“) sind dabei nicht so entscheidend wie die „Chancen“, die den Menschen die Möglichkeit geben, ihr Leben nach eigenen Vorstellungen und Wünschen zu gestalten. Die Verwirklichung der menschlichen Fähigkeiten ist daher von wesentlicher Bedeutung für die Erweiterung des Konzepts und der Evaluierung der menschlichen und sozialen Entwicklung. Menschliches Wohlergehen ist insofern viel mehr als die Summe der verschiedenen Ebenen des Wohlergehens, als es an die Präferenzen des Einzelnen und der Gesellschaft in Bezug auf Chancengleichheit, Bürgerrechte, Ressourcenallokation und Möglichkeiten der Weiterbildung geknüpft ist.

Der wirtschaftliche Wohlstand - das Ergebnis der wirtschaftlichen Produktion - ist eine wichtige Komponente des Wohlergehens. Als Messgröße der wirtschaftlichen Produktion weist das Bruttoinlandsprodukt (BIP) ${ }^{3}$ aber deutliche Grenzen auf. So erfasst das BIP die laufende Produktion der Konsum- und Investitionsgüter sowie der Dienstleistungen, die in den Konten der Volkswirtschaftlichen Gesamtrechnung erscheinen, nicht aber marktfremde Aktivitäten der privaten Haushalte ${ }^{4}$ (wie beispielsweise Kindererziehung) und Tätigkeiten wie die Erhaltung natürlicher Ressourcen, die durch Nettozugänge zum Kapitalstock einer Gesellschaft einen wesentlichen Beitrag zum Wohlergehen künftiger Generationen leisten ${ }^{5}$. Gesamtmessgrößen von Produktion und Einkommen, wie beispielsweise das BIP, können auch den Präferenzen der Gesellschaft in Bezug auf die soziale Gerechtigkeit nicht gebührend Rechnung tragen. 
Das BIP schließt auch Aktivitäten ein, die keinen direkten Beitrag zum Wohlergehen leisten.

Wir können keinen klaren Konsens hinsichtlich der Bedeutung des Begriffs Wohlergehen erwarten ...

... was aber Versuche zur Konzipierung summarischer Indikatoren nicht verhindert hat.

Diese Messgrößen deuten darauf hin, dass das Wohlergehen nicht mit der BIP-Entwicklung Schritt gehalten hat.
Das BIP umfasst auch Waren und Dienstleistungen, die nicht direkt zum Wohlergehen beitragen. Beispiele hierfür sind die so genannten „regrettables“, die etwa durch Umweltverschmutzung, Kriminalität und Scheidungen entstehen. Abbildung 1.1 veranschaulicht, dass diese ,gesellschaftlich nachteiligen Effekte" dem BIP, nicht aber dem Wohlergehen (einschließlich des wirtschaftlichen Wohlstands) zuzurechnen sind. Die "gesellschaftlich nachteiligen Effekte" werden in Form von Kosten und Ausgaben wiedergegeben, die nicht direkt zum Wohlergehen beitragen, dennoch aber als notwendig empfunden werden, wie z.B. Leistungen für die nationale Sicherheit (vgl. Anhang A).

Unser Verständnis von Wohlergehen oder menschlichem Wohlbefinden hängt von Wertvorstellungen ab, die von Mensch zu Mensch und Gruppe zu Gruppe unterschiedlich sind. Außerdem bestehen im Zusammenhang mit der Messung der Dimensionen des Wohlergehens technische Schwierigkeiten. Subjektive Aspekte des Wohlergehens, wie Äußerungen der Lebenszufriedenheit und des persönlichen Wohlbefindens, lassen sich nur schwer messen oder mit erklärenden Variablen in Verbindung bringen ${ }^{6}$. Die Definition sozialer Bedürfnisse setzt beispielsweise gewisse Festlegungen dahingehend voraus, welcher Wert den Bedürfnissen unterschiedlicher Gruppen der Zivilgesellschaft jeweils beigemessen wird. Ein gewisses Maß an Einkommensungleichheit ist u.U. wünschenswert, um zu gewährleisten, dass der Bevölkerung Arbeitsanreize geboten werden, und kann auch individuelle Präferenzen für einen bestimmten Lebensstil, Wohnort, Beruf bzw. ein ausgewogenes Verhältnis zwischen Freizeit und Arbeit oder ehrenamtlicher Tätigkeit und bezahlter Beschäftigung widerspiegeln. Zur Hervorhebung wesentlicher gesellschaftlicher Trendentwicklungen können verschiedene Indikatoren gewählt werden, wobei jedem dieser Indikatoren unterschiedliche Gewichtungen zugeordnet und unterschiedliche Bedeutungsgrade beigemessen werden (vgl. Anhang A).

Trotz dieser Schwierigkeiten wurde mehrfach der Versuch unternommen, summarische Indikatoren des Wohlergehens zu entwickeln. Ein Konzept (Osberg, 2001) bezieht sich ausschließlich auf den wirtschaftlichen Wohlstand und vereint vier große Kategorien von Indikatoren: $a$ ) die jeweiligen Pro-Kopf-Konsumströme, $b$ ) Veränderungen des (Natur- und Human-) Kapitalstocks, c) Veränderungen in der Einkommensverteilung sowie d) Entwicklung der wirtschaftlichen Risiken.

Bei verschiedenen Messgrößen des Wohlergehens scheint in einigen OECD-Ländern der Trend hinter dem Wachstum des Pro-Kopf-BIP zurückgeblieben zu sein (vgl. Anhang A). Aus Messungen von Osberg (2001) geht hervor, dass die Trendentwicklung des Pro-Kopf-BIP bis zu den achtziger Jahren in vielen OECD-Ländern eng der Entwicklung des wirtschaftlichen Wohlstands folgte und die Kluft zwischen beiden erst danach entstanden ist. Abbildung A.2 zeigt die entsprechenden Ergebnisse für fünf Länder. Analog hierzu deuten einige andere Messgrößen, denen ein breiteres Spektrum sozialer Indikatoren zugrunde liegt (z.B. der Index of Sustainable Economic Welfare und der Fordham Index of Social Health) für den Zeitraum seit Anfang bis Mitte der achtziger Jahre auf die gleiche Trendentwicklung hin. Zurückzuführen ist dieses Auseinanderklaffen vor allem auf die Umweltzerstörung, die steigende relative Armut sowie Einkommensungleichheiten in einigen OECD-Ländern. 
Die menschlichen und sozialen Fähigkeiten beeinflussen das Wohlergehen ...
Wirtschaftliche und soziale Trends sind miteinander verknüpft, allerdings nur bis zu einem gewissen Grad. Abbildung A.1 zeigt die Trendentwicklungen bei einer Reihe sozialer Anliegen für einige oder alle OECDLänder seit Mitte der siebziger Jahre bzw. für spätere Zeiträume. Hierbei handelt es sich um Indikatoren für die Veränderungen bei Einkommen, Armut und Erwerbsbeteiligung wie auch die sich wandelnden Familienstrukturen, Gesundheit, Demographie und Umwelt.

\subsection{Die Wechselbeziehungen zwischen Wohlergehen auf der einen und Human- und Sozialkapital auf der anderen Seite}

Die Rolle der verschiedenen Faktoren, die einen Einfluss auf das Wohlergehen haben, wie auch ihre komplexen Wechselbeziehungen untereinander werden in Abbildung 1.2 veranschaulicht.

Auf der Inputseite von Abbildung 1.2 stehen das Natur- und Sachkapital wie auch die ,menschlichen und sozialen Fähigkeiten“. Unter Humankapital sind das Wissen, die Fähigkeiten und die Gesundheit zu verstehen, die dem Einzelnen zu Eigen sind (vgl. die Definition in Kapitel 2).

Abbildung 1.2 Wichtigste Inputs für menschliches Wohlergehen und ihre Wechselbeziehungen

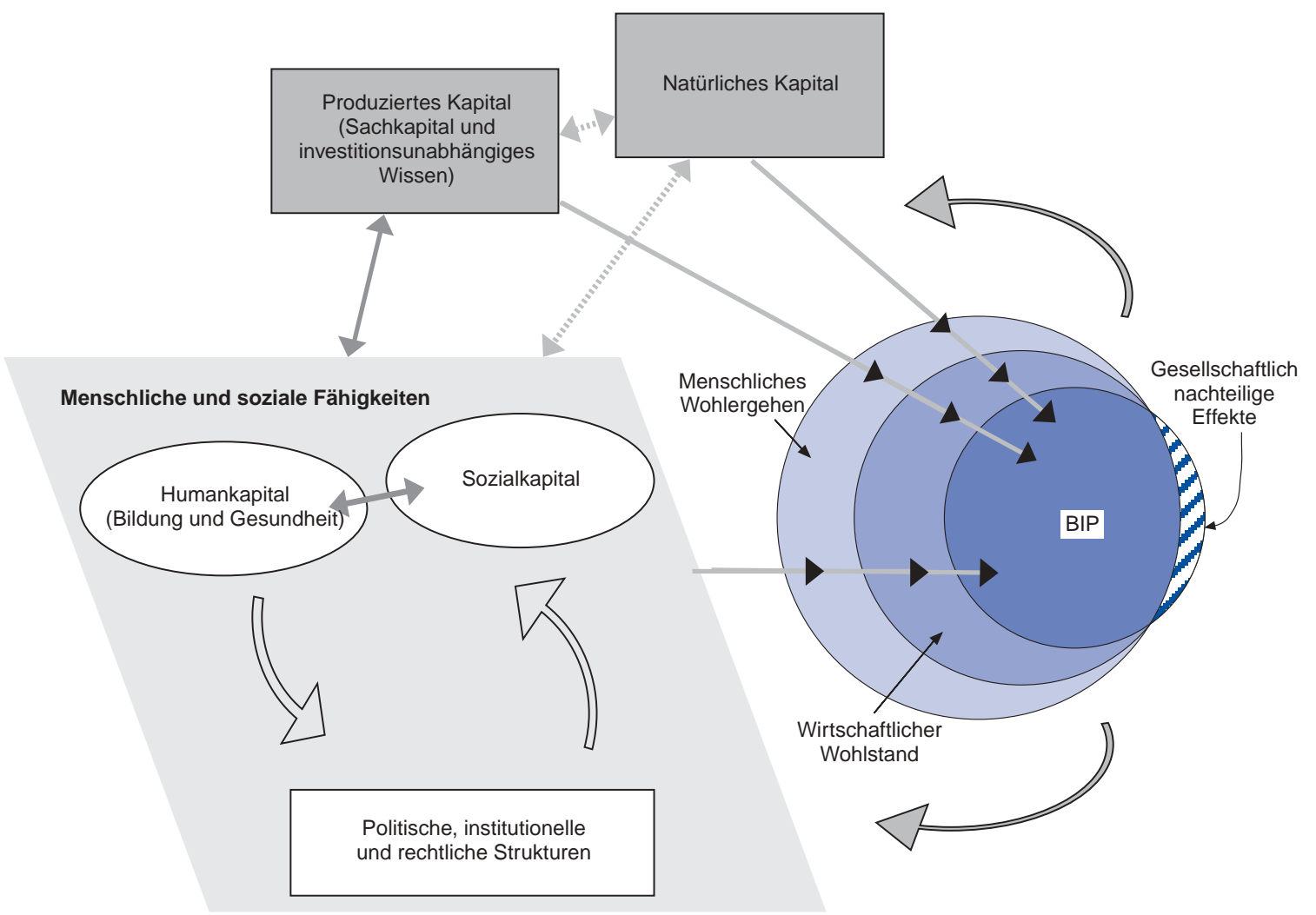

Quelle: OECD. 
... und die politischen, institutionellen und rechtlichen Strukturen sind äußerst wichtige Ergänzungen des Humanund Sozialkapitals.
Das Sozialkapital bezieht sich auf die Normen und Netzwerke, die die Zusammenarbeit in bzw. zwischen verschiedenen gesellschaftlichen Gruppen erleichtern (vgl. die Definition in Kapitel 3). Es besteht eine Wechselbeziehung zwischen den politischen, institutionellen und rechtlichen (PIR-)Strukturen und dem Human- und Sozialkapital, die das Wohlergehen beeinflussen. Außerdem gibt es noch spezielle direkte Beziehungen zwischen Humanund Sozialkapital sowie PIR einerseits und dem natürlichen und produzierten Kapital andererseits (diese sind allerdings in der Abbildung nicht wiedergegeben, um eine übermäßig komplexe Darstellung zu vermeiden).

Zwischen Humankapital, Sozialkapital und PIR besteht potentiell eine sehr starke Komplementarität ${ }^{7}$. Coleman (1988) hat in seinen Arbeiten über das Sozialkapital in den achtziger Jahren die Bedeutung starker Gruppenbeziehungen und enger Kontakte zwischen Eltern, Pädagogen und Schülern für die Förderung des Lernprozesses hervorgehoben. Außerdem können Bildung und Fortbildung die Entwicklung jener Gewohnheiten, Fähigkeiten und Wertvorstellungen begünstigen, die die soziale Zusammenarbeit und die Teilhabe am gesellschaftlichen Leben fördern. Leistungsfähige Einrichtungen, eine hoch qualifizierte Erwerbsbevölkerung und die Existenz von Normen und Netzwerken, die die soziale Kooperation erleichtern, dienen oft als Fundament für eine Erhöhung der Sachinvestitionen und können Strategien zur Regenerierung des natürlichen Umfelds verstärken. Auch die Gesundheit ist ein wichtiger Inputfaktor des Wohlergehens und der wirtschaftlichen Leistungsfähigkeit, gleichzeitig aber an Merkmale wie Alter, Lebensstil, gesellschaftlicher Status, Bildungsniveau und Ausmaß der sozialen Beziehungen und zwischenmenschlichen Solidarität geknüpft. Einige Ökonomen betrachten die Gesundheit sogar als Teil des Humankapitals ${ }^{8}$.

Die Marktaktivitäten und das Leben der Staatsbürger beruhen auf einer Vielzahl formeller und informeller bürgerlicher, politischer und rechtlicher Einrichtungen. Die Institutionen legen die Spielregeln fest. Rodrik (2000) beschreibt fünf Arten von Institutionen, die folgenden Zwecken dienen:

- Schutz von Privateigentum und Vertragstreue,

- Dämpfung gewisser Unternehmensaktivitäten,

- Stützung der makroökonomischen Stabilität,

- Bereitstellung einer Sozialversicherung bzw. eines sozialen Schutzes sowie

- Bewältigung sozialer Konflikte.

Wenn diese Einrichtungen effizient arbeiten, können sie es Ländern in verschiedenen Entwicklungsstadien ermöglichen, den Wandel aktiv zu gestalten und ein nachhaltiges Wirtschaftswachstum zu erreichen ${ }^{9}$.

Das Konzept des sozialen Zusammenhalts ist eng mit dem des Sozialkapitals verbunden. Jenson (1998) hat den sozialen Zusammenhalt als „die gemeinsamen Wertvorstellungen und Verpflichtungen einer Gemeinschaft“ definiert und fünf wichtige Dimensionen identifiziert: Zugehörigkeit, Integration, Partizipation, Anerkennung und Legitimität. Gesellschaften mit relativ starkem Zusammenhalt erreichen ihre gemeinsam aufgestellten Ziele, 
Es muss eine klare Unterscheidung vorgenommen werden zwischen Humankapital, Sozialkapital und den politischen, institutionellen und rechtlichen Strukturen.
Dauerhaftes Wohlergehen setzt angemessene Human- und Sozialkapitalinvestitionen voraus ... da sie von Ausgrenzung bedrohte einzelne Bürger und Gruppen besser schützen und integrieren. So stellt Ritzen (2001) fest: „Das Ziel des sozialen Zusammenhalts setzt die Verbindung einer Organisationsstruktur, die auf Marktkräften, Wahlfreiheit und Unternehmertum basiert, mit einer Verpflichtung auf Werte wie Solidarität und gegenseitige Unterstützung voraus, die für alle Mitglieder der Gesellschaft freien Zugang zu Vorteilen und Schutz gewährleistet". Diese Definition des sozialen Zusammenhalts beschreibt Ergebnisse bzw. Situationen der sozialen Harmonie, die von verschiedenen Faktoren hervorgebracht werden, darunter auch Human- und Sozialkapital. Das Konzept des sozialen Zusammenhalts ist also umfassender als das des Sozialkapitals.

Human- und Sozialkapital sind eng mit den Wirkungseinflüssen von Institutionen sowie politischen und sozialen Mechanismen auf die Gesellschaft verbunden. Allerdings müssen die verschiedenen Elemente sorgfältig voneinander getrennt werden, da:

- das Humankapital dem einzelnen Menschen zu Eigen ist,

- das Sozialkapital aus den gesellschaftlichen Beziehungen resultiert und

- die politischen, institutionellen und rechtlichen Regelwerke die Bestimmungen und Einrichtungen beschreiben, in deren Rahmen das Human- und Sozialkapital zum Einsatz kommt.

\subsection{Kann Wohlergehen von Dauer sein?}

Einige der Effekte des Human- und Sozialkapitals auf das Wohlergehen kommen erst langfristig zum Tragen, und dies gilt vor allem für Sozialinvestitionen in Kinder. Unzureichende Investitionen bergen die Gefahr, die Chancen der kommenden Generationen zu untergraben. Überlegungen über künftige „soziale Bedürfnisse“ sind eine große Herausforderung und zeigen, wie notwendig eine Koordinierung zwischen den verschiedenen Politikbereichen ist. Die politisch Verantwortlichen messen der nachhaltigen Entwicklung eine immer größere Bedeutung bei, was zeigt, dass sie sich in wachsendem Maß mit den Berührungspunkten zwischen Umweltpolitik und künftigen Bedürfnissen auseinander setzen.

Eine engere Verschränkung der Anliegen im Blick auf das ökonomische, soziale und ökologische Umfeld ist angebracht, da unabhängig von der gewählten Politik die gegenwärtigen Investitionsentscheidungen mit den langfristigen Trendentwicklungen, Kosten und Vorteilen in Verbindung gebracht und die Interdependenzen zwischen verschiedenen Prognosen berücksichtigt werden müssen. Im Sachstandsbericht über das OECDDreijahresprojekt für nachhaltige Entwicklung (OECD, 1999b) heißt es, dass „,er Begriff [der nachhaltigen Entwicklung] inzwischen insofern eine weiterreichende Bedeutung bekommen hat, als die Ziele Steigerung von wirtschaftlicher Effizienz und materiellem Wohlstand den Sozial- und Umweltproblemen in einem globalen Politikrahmen Rechnung tragen müssen“. 


\section{... da Veränderungen im sozialen und natürlichen Umfeld erst über einen längeren Zeitraum wirksam werden.}

Infolgedessen sind soziale Partnerschaften und ein Konsens für eine nachhaltige Entwicklung unerlässlich.
Jede Verschlechterung des sozialen Umfelds vollzieht sich wahrscheinlich schrittweise und betrifft einige Gruppen der Gesellschaft stärker als andere. Eine derartige Beeinträchtigung könnte die Form eines verstärkten Unsicherheitsgefühls, einer Zunahme antisozialer Verhaltensweisen, darunter auch von Kriminalität, oder auch einer Verlängerung der Fahrzeiten zwischen Wohnort und Arbeitsplatz sowie geringeren persönlichen Wohlbefindens annehmen ${ }^{10}$. Gewisse Aspekte dieser Verschlechterung, wie beispielsweise die Reduzierung des sozialen Engagements, treten im Anfangsstadium womöglich noch gar nicht offen zu Tage.

Der soziale Zusammenhalt kann eine Gesellschaft mobilisieren und in ihr die Energien wecken, die notwendig sind, um Probleme zu lösen. Laut Rodrik (1998) kann eine soziale Polarisierung die Reaktionskapazitäten einer Volkswirtschaft auf negative wirtschaftliche Schocks beeinträchtigen. Eine sich ausweitende Kluft zwischen hoch und minder qualifizierten Kräften kann den sozialen Zusammenhalt untergraben. Human- und Sozialkapital können eine wichtige Rolle spielen, wenn sie dazu beitragen, den effektiven Einsatz von Kompetenzen, den Informationsaustausch wie auch die Beilegung von Konflikten zu erleichtern. Dobell (2001, S. 37) hält fest: „Nachdem die politische Macht heute verstärkt von der Zivilgesellschaft und den demokratischen Institutionen ausgeht, können wirtschaftliche Entscheidungen, die erhebliche verteilungspolitische Folgen haben, nicht ausschließlich auf der Grundlage wirtschaftlicher Berechnungen oder einfach auf der Basis einer Kosten-Nutzen-Analyse bzw. anderer, sich daraus ergebender Kriterien durchgeführt werden. Die Akzeptanz (Rechtmäßigkeit) in den Augen einer mit erweiterten Machtbefugnissen ausgestatteten Zivilgesellschaft ist eine notwendige Voraussetzung für deren Zustimmung zur Wirtschaftspolitik und zum weiteren Streben nach Wirtschaftswachstum.“ 


\section{Anmerkungen}

1. Diese Anliegen sind nicht neu. Sie wurden bereits in den siebziger Jahren im Gefolge des beispiellosen Wirtschaftswachstums der unmittelbaren Nachkriegszeit offen formuliert. Die OECD startete in den siebziger Jahren ein Social Indicator Programme (Sozialindikatorenprojekt) im Anschluss an eine Ministererklärung aus dem Jahr 1970, in der hervorgehoben wurde, dass „Wachstum kein Selbstzweck ist, sondern vielmehr ein Instrument zur Schaffung besserer Lebensbedingungen“ und dass „... den qualitativen Aspekten des Wachstums wie auch der Definition von Politikmaßnahmen im Hinblick auf die wichtigen wirtschaftlichen und sozialen Entscheidungen, die es im Zusammenhang mit der Allokation wachsender Ressourcen zu treffen gilt, größere Aufmerksamkeit geschenkt werden muss“ (zitiert in OECD, 1976, S. 7).

2. Der wirtschaftliche Wohlstand ist im Begriff „Wohlergehen“ voll und ganz enthalten. Im weiteren Verlauf des vorliegenden Berichts bezieht sich der Begriff „Wohlergehen“ auf das menschliche Wohlergehen, sofern das Konzept nicht auf die wirtschaftlichen Aspekte begrenzt ist. In diesem Fall wird dann der Begriff ,wirtschaftlicher Wohlstand“ verwendet.

3. Die Grenzen der bestehenden, auf dem BIP beruhenden Messgrößen wurden von den an der Ausarbeitung von Systemen der Volkswirtschaftlichen Gesamtrechnung Mitte des 20. Jahrhunderts Beteiligten anerkannt.

4. „Der Wert der Frauenarbeit im eigenen Haushalt ist hier nicht berücksichtigt worden, da es für diese Arbeit keinen Markt gibt - ein Irrtum, der mit rd. 8 Bill. \$ zu Buche schlägt“ (Picciotto, 1998).

5. Das BIP umfasst Bruttoinvestitionen in Sachkapital wie auch den laufenden Konsum. Allerdings bleibt der Beitrag der laufenden Produktion zu Veränderungen in anderen Formen des Kapitalstocks generell unberücksichtigt.

6. In Kapitel 2 und 3 werden indessen neuere Analyseergebnisse über das berichtete Niveau des subjektiven Wohlergehens oder der Lebenszufriedenheit vorgestellt, die den Schluss nahe legen, dass einige der Faktoren, die das subjektive Wohlergehen mitbestimmen, empirisch ermittelt werden können.

7. In einigen Fällen sind Humankapital, Sozialkapital und PIR substituierbar, wenn beispielsweise amtliche Institutionen und Regeln an die Stelle informeller sozialer Netze treten können.

8. Gary Becker, der als einer der ersten den Begriff „Humankapital“ verwendete, sah in der Bildung, der Fortbildung am Arbeitsplatz (Training on the Job) und der Gesundheit Komponenten des Humankapitals, die Auswirkungen auf die Arbeitsverdienste und die wirtschaftliche Produktivität haben (S. 54-55 in Becker, 1993).

9. Wie Rodrik aber hervorhebt, sind die Empfehlungen für jedes Land unterschiedlich, da es kein Patentrezept gibt, das auf jedes Land bzw. jede Situation zugeschnitten werden kann.

10. Die Frage, ob sich der Gesundheitszustand der Menschen verschlechtert hat, ist ein separates Problem. Allein die Tatsache, dass viele Menschen den Eindruck haben, ihr Wohlbefinden habe sich verschlechtert, kann ausreichen, um auf eine Besorgnis erregende Entwicklung hinzudeuten. 



\section{DIE ROLLE DES HUMANKAPITALS}

„Für die Beständigkeit von Wachstum und Wohlstand bedarf es des sozialen Zusammenhalts; auch hier spielt das Humankapital eine entscheidende Rolle. Diese Auffassung wird inzwischen in immer breiteren Kreisen vertreten" (OECD, 1998, S. 91).

„Die Bildung übt zwar einen Einfluss auf die Arbeitsproduktivität aus, sie bezieht ihre Daseinsberechtigung aber nicht allein aus dieser Tatsache. Man lehrt Schülern und Studenten Bürgerkunde, Kunst oder Musik nicht einfach nur, um deren Arbeitsproduktivität zu steigern, sondern auch um ihr Leben zu bereichern und sie zu bewussteren Bürgern zu machen"(Weiss, 1995).

\subsection{Einleitung}

Wissen und Kompetenzen gewinnen wirtschaftlich zunehmend an Bedeutung ...

... und dabei sind die sozialen Auswirkungen des Lernens genauso wichtig wie die wirtschaftlichen.
Im Zuge der sich wandelnden wirtschaftlichen und sozialen Rahmenbedingungen spielen Wissen und Kompetenzen - das Humankapital - für den wirtschaftlichen Erfolg von Nationen und Individuen eine immer entscheidendere Rolle. Faktoren wie die Informations- und Kommunikationstechnologien, die Globalisierung der Wirtschaftstätigkeit wie auch der Trend zu größerer persönlicher Verantwortung und Autonomie haben allesamt die Lernanforderungen verändert. Die Schlüsselrolle, die Kompetenzen und Wissen bei der Förderung des Wirtschaftswachstums zukommt, ist von Ökonomen und anderen Experten weithin anerkannt worden.

Der nicht ökonomische Lernertrag in Form einer Verbesserung des persönlichen Wohlergehens und stärkeren sozialen Zusammenhalts wird von vielen Experten als genauso wichtig empfunden wie die Auswirkungen des Lernens auf Arbeitseinkommen und Wirtschaftswachstum. Diese persönlichen und sozialen Ziele des Lernens sind mit dem Ziel der Förderung der wirtschaftlichen Leistungsfähigkeit nicht zwangsläufig unvereinbar, zumal es zur Verwirklichung der wirtschaftlichen Wachstumsziele ausgeglichener, flexibler und anpassungsfähiger Menschen bedarf, die bereit sind, ein Leben lang weiter zu lernen.

In diesem Kapitel soll definiert und anhand von Beispielen veranschaulicht werden, was unter Humankapital zu verstehen ist. Ferner wird dessen Bedeutung im Zusammenhang mit einigen grundsatzpolitischen Kernfragen untersucht, darunter:

- „Was funktioniert“, um Lernerfolge zu fördern (Abschnitt 2.4)?

- Welche Auswirkungen haben verschiedene Lernformen (Niveau, Qualifikationsbereich oder -art) auf das BIP-Wachstum (Abschnitte 2.7 und 2.8)? 
- Wird zu wenig in Humankapital investiert, da Einigen die mit ihren Investitionen verbundenen sozialen Vorteile nicht voll zugute kommen?

- Welche Auswirkungen haben Bildung und Fortbildung auf das Wohlergehen (Abschnitte 2.7 und 2.8)?

\section{Humankapital wird definiert als das Wissen und die Kompetenzen, über die ein Mensch verfügt ...}

... und Eigenschaften.

\subsection{Was ist unter Humankapital zu verstehen?}

In der Vergangenheit wurden von den Ökonomen drei Produktionsfaktoren unterschieden: Grund und Boden, Arbeit und Sachkapital. Seit Anfang der sechziger Jahre richtete sich die Aufmerksamkeit immer stärker auf die Qualität des Faktors Arbeit, insbesondere dem Bildungs- und Ausbildungsniveau der Erwerbsbevölkerung. So entstand das Konzept des Humankapitals, das sich auf Kompetenzen und sonstige Eigenschaften des Einzelnen erstreckt, die eine Reihe persönlicher, wirtschaftlicher und sozialer Vorteile bringen. Qualifikationen und Kompetenzen werden weitgehend durch Lernen und Erfahrung erworben, können aber auch angeborene Fähigkeiten widerspiegeln. Gewisse Motivations- und Verhaltensaspekte wie auch persönliche Eigenschaften, so die körperliche, emotionale und geistige Verfassung eines Menschen, gehören ebenfalls zum Humankapital. Im vorliegenden Bericht wird folgende Definition des Humankapitals verwendet:

Wissen, Fähigkeiten, Kompetenzen und sonstige Eigenschaften von Individuen, die für das persönliche, soziale und wirtschaftliche Wohlergehen relevant sind.

Auch wenn das „Humankapital“ in der Vergangenheit oft in Bezug auf erworbene kognitive Kompetenzen und explizites Wissen definiert und gemessen wurde, gibt eine umfassendere Betrachtungsweise des Humankapitals, die auch menschliche Eigenschaften umfasst, angemessener wieder, wie verschiedene nicht kognitive Kompetenzen und sonstige Eigenschaften zum Wohlergehen beitragen und vom externen Umfeld, namentlich Lernprozessen, beeinflusst und verändert werden können. Humankapital entwickelt sich in einem spezifischen kulturellen Umfeld ${ }^{1}$.

Der Lernprozess und der Wissens- und Kompetenzerwerb beginnen mit der Geburt und enden mit dem Tod. Das Konzept des lebensbegleitenden Lernens hebt nicht nur die Bedeutung der Erwachsenenbildung und -fortbildung hervor, sondern auch das Lernen in allen Lebensabschnitten, darunter auch die Phase der „Anleitung zum Lernen als solchem“ (Learning to learn) in Schulen und anderen formellen Bildungseinrichtungen - sowohl lebensbegleitend als auch „lebensumfassend“. Humankapital entwickelt sich in folgenden Kontexten:

- Lernen in der Familie und im Rahmen verschiedener Betreuungsformen für Kleinkinder;

- formelle Bildung und Fortbildung, etwa im Kindergarten, im Rahmen der Schulpflicht, der beruflichen oder allgemeinen Fortbildung nach Ende der Schulpflicht, durch Hochschulausbildung, öffentliche Arbeitsmarktprogramme, Erwachsenenbildung usw.; 


\section{... und das Human- kapital hat viele Facetten ...}

\author{
... darunter Qualitäten, \\ die von Lese- und \\ Schreibkompetenzen \\ bis zur Ausdauer \\ reichen.
}

- Ausbildung am Arbeitsplatz wie auch informelles Lernen im Berufsleben durch spezifische Aktivitäten wie Forschung und Innovationen oder Teilnahme an verschiedenen professionellen Netzwerken;

- informelles Lernen am Arbeitsplatz sowie im Alltag durch die Teilhabe am Leben der Gesellschaft.

Mit dieser umspannenden Vision des Humankapitals kann der Kritik begegnet werden, der zufolge das Konzept des Humankapitals seiner menschlichen Qualität beraubt wird, da Menschen mit Funktionsmechanismen gleichgestellt würden. Dagegen vertrat die OECD in ihrem Bericht über das Humankapital von 1998 die Auffassung, dass das Konzept des Humankapitals ,wirkungsvoll hervorhebt, wie wichtig Menschen in einer wissens- und kompetenzbasierten Wirtschaft geworden sind“.

Das Humankapital ist von Natur aus facettenreich. Qualifikationen und Kompetenzen (wie die Lese-, Schreib- und Sprechfähigkeit) können allgemeiner Natur oder auch hochspezifisch und dem jeweiligen Kontext mehr oder minder gut gerecht werden. Firmenspezifische Kompetenzen und Kenntnisse werden durch das Lernen am Arbeitsplatz und betriebsinterne Schulungen erworben. Ein Großteil des Wissens und der Qualifikationen wird mittlerweile stillschweigend vorausgesetzt und ist nicht kodifiziert oder in Dokumentationen nachlesbar, entweder weil eine Kodifizierung unmöglich ist oder einfach nicht vorgenommen wurde. Je mehr Wissen implizit und nicht explizit ist, desto schwerer lässt es sich austauschen und vermitteln. Lundvall und Johnson (1994) stufen Wissen in vier Kategorien ein:

1. Know-what (,wissen, was“): diese Form bezieht sich auf das Wissen über „Fakten“;

2. Know-why (,wissen, warum“): diese Form bezieht sich auf das Wissen über die Regeln und Gesetze, denen die Natur, menschliche Intelligenz und Gesellschaft folgen;

3. Know-how (,wissen, wie“): diese Form bezieht sich auf die Qualifikationen (d.h. die Fähigkeit, Aufgaben zu erledigen);

4. Know-who (,wissen, wer"): diese Form befasst sich mit der sozialen Fähigkeit, mit verschiedenartigen Menschen und Experten zu kooperieren und zu kommunizieren.

Im Gegensatz zum Sachkapital ist Humankapital Bestandteil des Menschen $^{2}$. Das Humankapital wächst mit seiner Nutzung und der gesammelten Erfahrung, sowohl innerhalb als auch außerhalb des Arbeitsumfelds wie auch in informellen und formellen Lernprozessen, doch büßt das Humankapital in der Regel auch an Wert ein, wenn es nicht zum Einsatz kommt. Gewisse Qualifikationen verlieren mit zunehmendem Alter ihre Bedeutung, und dieser Prozess erklärt z.T. den ab einem bestimmten Alter beobachteten Abwärtstrend beim gemessenen Humankapital (oder zumindest dem mit einem bestimmten Bildungsniveau verbundenen Mehrverdienst) (Mincer, 1974). So kann das Humankapital nicht als ein homogenes und statisches Bündel von Qualifikationen bzw. Kompetenzen betrachtet werden, die Menschen ein für allemal erworben haben. Einige der Qualifikationen und persönlichen Eigenschaften, die für das Humankapital relevant sind, lassen sich in folgende Kategorien einteilen: 
1. Kommunikation (darunter Fremdsprachenkompetenzen in den unmittelbar nachstehenden Rubriken)

- Hören

- Sprechen

- Lesen

- Schreiben

2. Rechenfertigkeiten

3. Persönliche Kompetenzen

- Motivation/Ausdauer

- „Lernen lernen“ und Selbstdisziplin (einschließlich autodidaktischer Lernstrategien)

- Urteilsvermögen auf der Basis eines relevanten Katalogs ethischer Wertvorstellungen und Lebensziele

4. Kompetenzen im zwischenmenschlichen Bereich

- Teamarbeit

- Führungskompetenzen

5. Sonstige Qualifikationen und Eigenschaften (die für viele der oben stehenden Bereiche von Nutzen sind)

- Vertrautheit mit Informations- und Kommunikationstechnologien

- Implizites Wissen

- Problemlösung (gehört auch zu anderen Qualifikationen)

- Körperliche Merkmale und Gewandtheit

In der Praxis erfordern die meisten menschlichen Aktivitäten Teamarbeit.
Fukuyama (1995) stellt fest, dass ,praktisch alle wirtschaftlichen Aktivitäten ... nicht von Individuen, sondern von Organisationen durchgeführt werden, die ein hohes Maß an sozialer Zusammenarbeit erfordern“. Unter „Organisationskapital“" werden das gemeinsame Wissen, Teamarbeit und Verhaltensnormen sowie Interaktionen innerhalb von Organisationen verstanden. Es handelt sich hierbei um eine Form des Sozialkapitals auf Organisationsebene, die im nachstehenden Kapitel erörtert wird. Leana und Van Buren (1999) definieren das Organisationskapital als „Ressource, die den Charakter der sozialen Beziehungen innerhalb eines Unternehmens widerspiegelt“. Dieses Kapital entwickelt sich in dem Maße, wie ,jedes Mitglied - auf seinem Niveau einen Beitrag zur Verwirklichung des gemeinsamen Ziels in einem Klima des Vertrauens leistet, wobei Werte dadurch entstehen, dass gemeinsamen Aktionen zum Erfolg verholfen wird“. Die Beteiligten werden zur Zusammenarbeit ermutigt, wenn die Organisationsstruktur die Möglichkeiten für opportunistisches Verhalten begrenzt, Vertrauen weckt und festigt, der offenen Kommunikation Vorschub leistet und gemeinsame Ziele und Wertvorstellungen fördert (z.B. Axelrod, 1984). 
Wissen und Netzwerke spielen eine zunehmend wichtige Rolle.
Es lässt sich nur schwer eine Summe der verschiedenen Humankapitalkomponenten bilden ...
... und bei der Messung des Humankapitals muss den Grenzen vieler Indikatoren Rechnung getragen werden.
Es hat sich immer mehr die Erkenntnis durchgesetzt, dass Wissensmanagement und -austausch wegen der grundlegend neuen Organisationsstruktur und technischen Bedingungen in der so genannten ,neuen Wirtschaft“ von entscheidender Bedeutung sein können (Lesser, 2000). Wissen, Flexibilität, Vertrauen und Vernetzung spielen bei der Suche nach neuen Ideen und Verfahrensweisen in einem sich rasch wandelnden wirtschaftlichen Umfeld eine immer größere und wichtigere Rolle. Viele Beobachter haben das Konzept der „,neuen Wirtschaft“ mit dem der „Lernwirtschaft“ verknüpft, in der die Kapazität von Netzwerken, Unternehmen und einzelnen Menschen, Wissen zu erwerben, auszutauschen, zu vermitteln und anzuwenden, heute qualitativ wichtiger ist als zuvor (OECD, 2000a).

\subsection{Wie kann Humankapital gemessen werden?}

Formelle Bildungsnachweise sind einfache und ohne weiteres messbare Indikatoren für Qualifikationen und Kompetenzen. Ihr Nachteil besteht darin, dass sie dem durch informelle Lernprozesse oder Erfahrung erworbenen Humankapital nicht gebührend Rechnung tragen und sich die unterschiedlichen Bildungsabschlüsse (beispielsweise aus verschiedenen Ländern) nur schwer vergleichen lassen. Eine Alternative wäre die Verwendung von Fragebogen zur Messung der Schüler- und Studentenleistungen bzw. Erwachsenenkompetenzen, wie dies im Rahmen der PISA-Schulleistungsstudie (Programme for International Student Assessment) und der Internationalen Erhebung über Grad und Verteilung elementarer Grundqualifikationen Erwachsener (IALS - International Adult Literacy Survey) erfolgt (vgl. Abb. 2.1). Die Programme messen nur einige Aspekte der Qualifikationen und Kompetenzen und stoßen an Grenzen, die durch die Umfrage- und Evaluierungsmethoden selbst bedingt sind (beispielsweise in Bezug auf die Größe des Stichprobenumfangs, das Spektrum der berücksichtigten interdependenten Variablen wie auch die erfassten Länder).

Es sind Versuche unternommen worden, den Wert des Humankapitals anhand von Gesamtdaten projizierter Arbeitseinkommen von Individuen nach dem Niveau der Erstausbildung zu schätzen ${ }^{3}$. Bei einem derartigen Ansatz wird die Bedeutung von „kollektivem Wissen oder Know-how“, über das Organisationen und andere kollektive Einrichtungen verfügen, überhaupt nicht berücksichtigt ${ }^{4}$. (Prinzipiell gelingt es Einzelpersonen fast nie, für ihre hausinternen Kompetenzen in ihrem Arbeitsverdienst eine volle ökonomische Rente zu erzielen, da ihr Arbeitgeber ja der einzige potentielle Käufer dieser Kompetenzen ist.) Bei dieser Aggregationsmethode werden zudem die Auswirkungen von Wechselbeziehungen und Übergreifeffekten ${ }^{5}$, die durch die Verbesserung des Humankapitals einiger Mitarbeiter entstehen, wahrscheinlich nicht erfasst. Schließlich lassen sich die sehr spezifischen, kulturgebundenen, nicht vermittelbaren, impliziten und heterogenen Dimensionen des Humankapitals nicht einfach in derartige Gesamtmessgrößen des Humankapitals einbeziehen.

Diese Vorbehalte weisen darauf hin, dass bei Schätzungen von Bestand und Volumen der Humankapitalinvestitionen, insbesondere bei Vergleichen auf internationaler Ebene, erhebliche Vorsicht geboten ist. Humankapitalindikatoren, denen nur ein Index zugrunde liegt, müssen durch spezifischere Messgrößen ergänzt werden, die auf direkten Messungen des Wissens und der Qualifikationen in Organisationen beruhen. 
Abbildung 2.1 Komparative Verteilung des Grundbildungsniveaus

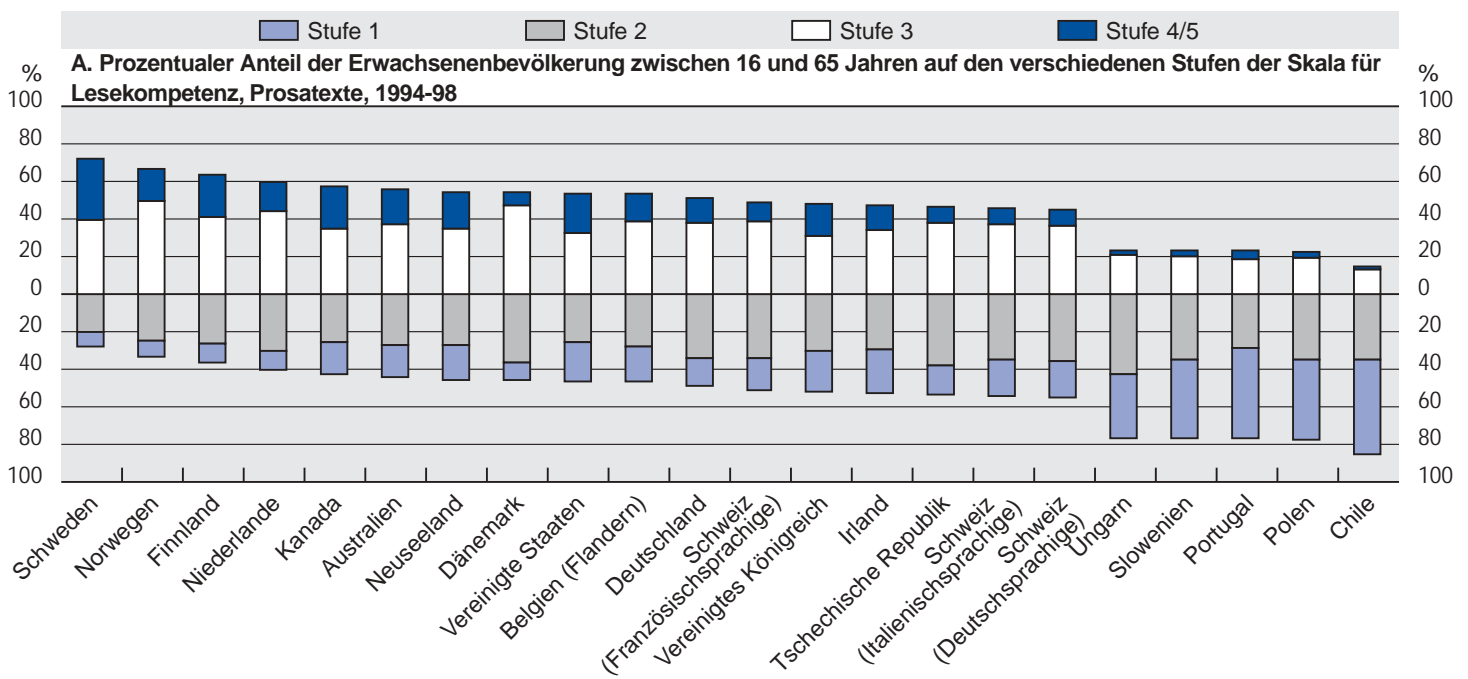

\% B. Prozentualer Anteil der Erwachsenenbevölkerung zwischen 16 und 65 Jahren auf den verschiedenen Stufen der Skala für 100 Lesekompetenz, Sachtexte, 1994-98

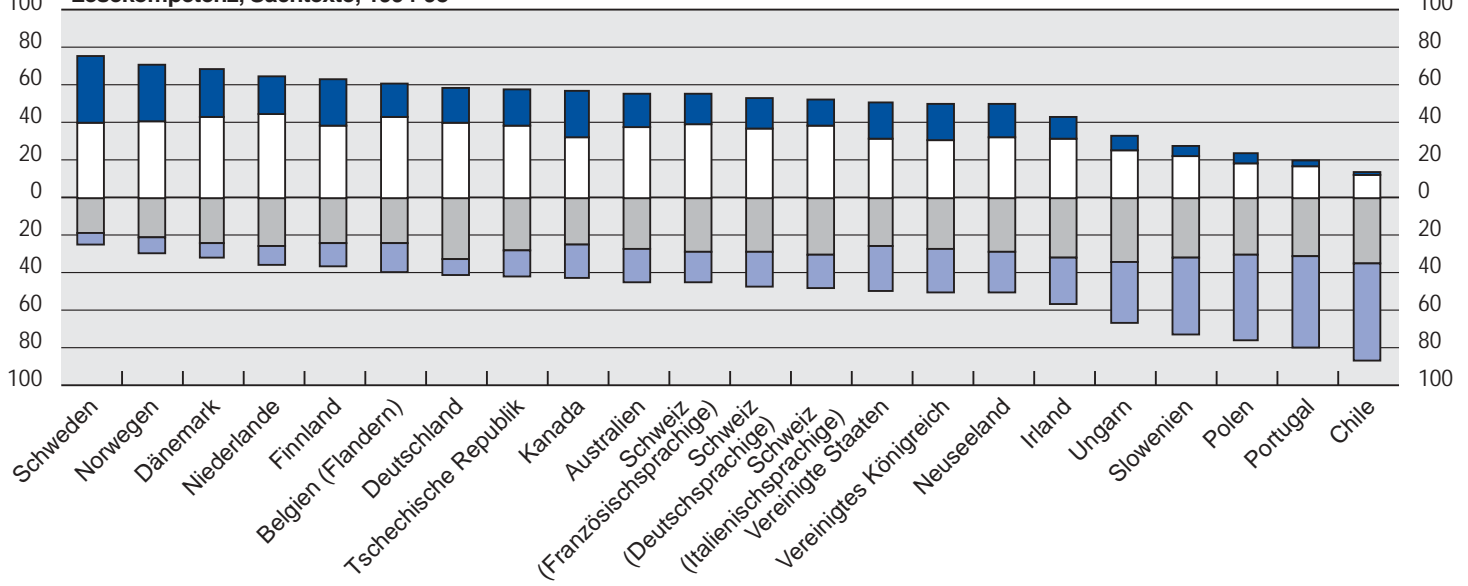

\% C. Prozentualer Anteil der Erwachsenenbevölkerung zwischen 16 und 65 Jahren auf den verschiedenen Stufen der Skala für

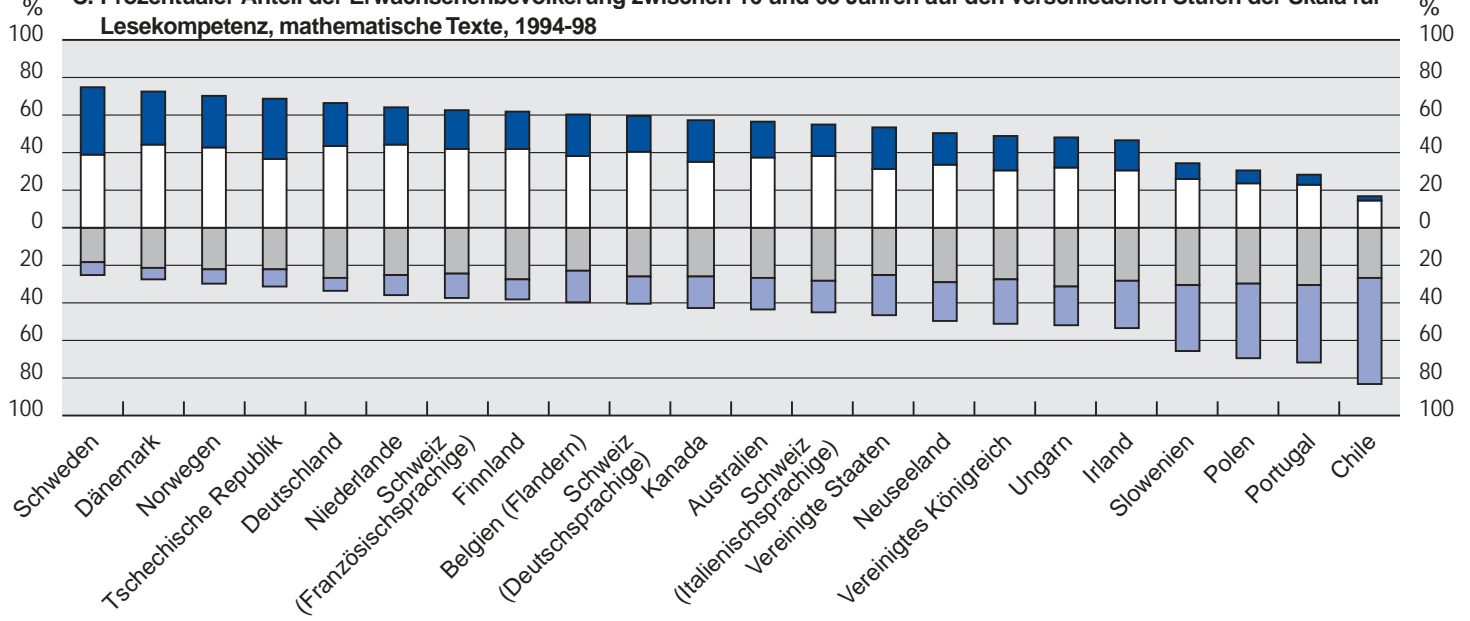

Anmerkung: Die Rangfolge der Länder wird durch den Anteil an Personen auf den Stufen 3 bzw. 4/5 bestimmt. Quelle: OECD (2000), Literacy in the Information Age, Paris. 


\subsection{Wie wird Humankapital gebildet?}

Investitionen in Qualifikationen finden in vielen verschiedenen Konstellationen und Stadien des Lebenszyklus statt ...

... und der kulturelle Kontext beeinflusst den Lernprozess.

\section{Zur Leistungs- steigerung muss der Ausgabenanstieg für den Bildungs- bedarf durch andere Strategien flankiert werden.}

In Ländern mit höherem Niveau der Wirtschaftsentwicklung ist die Rendite der Bildungsausgaben möglicherweise rückläufig.
Humankapitalbildung findet nicht nur im Rahmen der formellen Bildung oder in Aus- und Fortbildungsprogrammen statt, sondern auch durch die nicht formelle Interaktion mit anderen sowie persönliche Reflexion und autodidaktisches Lernen. Die sozialen Netzwerke und Normen spielen während des gesamten Lebenszyklus bei der Förderung einer Lernkultur eine wichtige Rolle. In diesem Abschnitt werden empirische Belege für den Einfluss verschiedener Faktoren auf die Lernergebnisse untersucht - Informationen, die hauptsächlich auf der Ebene der Primar- und Sekundarschulbildung verfügbar sind; er basiert auf eine sehr umfangreiche Fachliteratur zu verschiedenen Disziplinen. Weitere Einzelheiten finden sich in den Anhängen C und D.

Die Bedeutung des kulturellen Rahmens, in dem Lernen und Unterricht stattfinden, ist vielfach hervorgehoben worden. Fuller und Clarke (1994) untersuchen zur Evaluierung der Produktivität in Schulen beispielsweise eine Vielzahl unterschiedlicher Konzepte, darunter Ansätze, die sie als Policymechanics ${ }^{6}$ - und Classroom-culturalist ${ }^{7}$-Konzepte bezeichnen. Neben den Normen und Einstellungen, die Schüler und Studenten von zu Hause und ihrem sozialen Hintergrund übernehmen, erwerben sie bestimmte Normen, Gewohnheiten und Wertvorstellungen im kulturellen Kontext ihrer Schule und Klasse. Diese Normen bekräftigen oder verändern ihre Vorstellungen in Bezug auf Verdienst, Status, Verhaltens- und Teilnahmenormen wie auch ihre Einstellung dem Lernen gegenüber. So spielen die kulturell begründeten Bedeutungen des Lernens und der Unterrichtspraktiken und -instrumente eine ebenso große Rolle wie der materielle Gehalt der schulischen Inputs bzw. das Volumen der bereitgestellten Ressourcen. Fuller und Clarke machen geltend, dass der kulturalistische Ansatz zwar eine durchaus berechtigte Perspektive darstellt, er aber weder in der Lage war, empirische Belege für kulturübergreifende Interpretationsunterschiede in Bezug auf die den Unterrichtsinstrumenten und -praktiken jeweils beigemessene Bedeutung zu liefern noch Handlungsoptionen zu ermitteln, die dem Lernprozess in einem jeweils unterschiedlichen kulturellen Umfeld Impulse verleihen könnten.

In den OECD-Ländern haben die öffentlichen Bildungsausgaben auf allen Ebenen in den vergangenen Jahren zugenommen, was sich sowohl aus den steigenden Teilnahmequoten (insbesondere im Zeitraum nach Ende der Schulpflicht) als auch aus den in realer Rechnung je Schüler wachsenden Ausgaben erklärt - die ihrerseits oft durch die Verkleinerung der Schulklassen bedingt sind. Aus dem vorliegenden Datenmaterial geht hervor, dass zwischen Ausgabenniveau und Unterrichtsergebnissen zwar ein Zusammenhang besteht, dass dies jedoch nur bis zu einem gewissen Grad der Fall ist und die Ausgabeneffizienz sehr stark davon abhängt, wie die Unterrichtsmethoden, die schulische Organisation und elterliche Unterstützung mit der Bereitstellung zusätzlicher Mittel für den Unterricht kombiniert werden (Hanushek und Kim, 1995; Hanushek und Kimko, 2000; und Gundlach, Wossmann und Gmelin, 2000 ${ }^{8}$ ).

Manche Belege deuten darauf hin, dass die Rendite der Ausgaben für die formelle Bildung in wirtschaftlich höher entwickelten Ländern schrumpfen könnte (Hanushek und Kim, 1995). Für die Entwicklungsländer haben Fuller und Heyneman (1989) gezeigt, dass in einem von Verarmung geprägten Milieu die Schule eine prägendere Rolle für die schulischen Leistungen spielt als der familiäre Hintergrund, so dass der staatlichen Politik bei der Beeinflussung des 


\section{Kleinere Klassen bringen offenbar bessere Ergebnisse, doch halten sich diese Effekte in Grenzen.}

Lernerfolgs der am stärksten Benachteiligten eine größere Bedeutung beigemessen werden kann. Eine wichtige Schlussfolgerung, die sich aus vielen empirischen Arbeiten ergibt, lautet, dass ein Ausgabenanstieg, der auf eine Expansion der Schulbesuchsquoten (und insbesondere einen Rückgang der Abbrecherquoten) zurückgeht, generell höhere Renditen (d.h. höhere Arbeitseinkommen) erbringt als eine Erhöhung der Ausgaben je Schüler und Schuljahr. Diese Feststellung gilt offenbar für die Vereinigten Staaten (Betts und Roemer, 1998) und die Entwicklungsländer gleichermaßen (Psacharopolous, 1994).

Ein Großteil der zusätzlichen Bildungsausgaben wurde in den vergangenen Jahrzehnten für die Reduzierung der Klassengrößen aufgewendet. Nun stellt sich die Frage, ob sich weitere Klassenreduzierungen in Zukunft auszahlen werden. Die Effekte hängen vom Alter und von den Bedürfnissen der jeweiligen Gruppen ab - so kann eine Reduzierung der Klassenstärke bei kleinen Kindern beispielsweise positive Auswirkungen haben (im Kindergarten oder in der ersten Grundschulklasse). In den Vereinigten Staaten und anderen OECD-Ländern durchgeführte Forschungsarbeiten legen auf der Grundlage von Schülertestergebnissen den Schluss nahe, dass kleinere Klassen etwas bessere Schulleistungen erbringen. Unabhängig davon müssen die infolge einer Verringerung der Klassenstärke erreichten Verbesserungen gegen die Kosten und Vorteile alternativer Strategien aufgewogen werden, wie beispielsweise eine Verstärkung der Bemühungen um die Verbesserung der Lehrerausbildung und -qualität. Eine wesentliche Reduzierung der Klassenstärke, so z.B. von 25 auf 15, wird voraussichtlich zu einer gewissen Verbesserung der Unterrichtsergebnisse führen, allerdings zu enormen Kosten. So ist die Wahrscheinlichkeit groß, dass in bestimmten Fächern, für bestimmte Schülergruppen und bei spezifischen Anpassungen der Unterrichtsmethoden mit kleineren Klassen bessere Ergebnisse erzielt werden könnten. Diese Möglichkeit muss aber in Verbindung mit der Politikoption, für bestimmte Zwecke kleinere und für andere größere Klassen zu wählen, noch weiter untersucht werden.

Es besteht in breiten Kreisen Einvernehmen darüber, dass Familie, sozialer Hintergrund und Elternhaus die Effektivität des Schulbesuchs beeinflussen. In vielen Studien wird die Bedeutung verschiedener Faktoren hervorgehoben, so z.B. die Unterstützung, die Eltern ihren Kindern geben, bzw. die Wünsche und Hoffnungen sowie Arbeitsmethoden, die sie ihnen vermitteln. So identifiziert Wossmann (2000) beispielsweise unter Verwendung von Daten für eine Vielzahl von Ländern, die an der Third International Mathematics and Science Survey (TIMSS - Dritte Internationale Erhebung über Mathematik- und naturwissenschaftliche Kenntnisse) teilgenommen haben, dass der Einfluss der Eltern auf den Unterricht ihrer Kinder bzw. das Elterninteresse wie auch Lehrplanentscheidungen für die Unterrichtsergebnisse eine wichtige Rolle spielen. Bourdieu (1979) verwendet den Begriff des „Kulturkapitals“ zur Beschreibung der Gewohnheiten und kulturellen Praktiken, denen das Wissen und Verhalten zugrunde liegt, das im Kontakt mit Rollenvorbildern innerhalb und Einflüssen außerhalb der Familie erworben wurde. Ferner steht das Kulturkapital für die Gesamtheit des familiären Erbes, darunter das Bildungsniveau der Eltern, ihr sozialer Status wie auch Verhaltensweisen, Normen und Praktiken innerhalb der Familie, die den Schulerfolg beeinflussen. Es wurden fünf große Kategorien identifiziert (Kellaghan et al., 1993): Arbeitsgewohnheiten der Familie, schulische Unterstützung und Orientierung, Anreize zum Nachdenken und Diskutieren über Gedanken und Ereignisse, sprachliches Umfeld (die Möglichkeit, seinen Gedanken und seiner Phantasie Ausdruck zu verliehen) sowie die 


\section{Soziale Netzwerke spielen beim Lernen eine wichtige Rolle.}

\section{Partnerschaften, Finanzierung und ein effizienter Regulie- rungsrahmen spielen eine Rolle.}

\section{Die Erwachsenen- bildung muss sorgfältig definiert werden, wenn sie effektiv sein soll.}

Wünsche und Erwartungen der Eltern in Bezug auf die Ausbildung der Kinder' Die Eltern oder Geschwister können Kindern bei den Hausaufgaben helfen und ihnen die Freude am Lernen und Disziplin vermitteln. Je höher die Erwartungen der Eltern (und insbesondere der Mutter) sind, desto geringer ist die Wahrscheinlichkeit des Schulabbruchs. Alle diese Faktoren sind potentiell effiziente Puffer gegen die negativen Einflüsse eines niedrigen sozioökonomischen Status und eines geringen Bildungsniveaus der Eltern auf die Schulleistungen der Kinder (White und Kaufman, 1997).

Coleman, auf den die Einführung des Konzepts des Sozialkapitals in die Bildungsforschung in erster Linie zurückgeht, hat die Bedeutung des Erwachsenenumfelds hervorgehoben, das die jungen Menschen umgibt, die in die jeweiligen Gruppen der ihnen am nächsten stehenden Erwachsenen ,eingebettet" sind (Coleman, 1988). Unter Sozialkapital werden hier die Ressourcen verstanden, die dank der sozialen Beziehungen, der Zugehörigkeit zu Netzwerken und dem Rückgriff auf gemeinsame Normen erworben wurden. Das Kulturkapital hingegen - das eine Dimension des Sozialkapitals darstellt - bezieht sich auf die Ressourcen, die den Familien zu Eigen sind und durch die Menschen einen bestimmten sozialen Status erlangen (Bourdieu, 1979; Bourdieu und Passeron, 1970). Die Familien und Schüler haben Zugang zu mehr oder minder großen Anteilen am Finanz-, Human-, Kultur- und Sozialkapital, doch ist dieses Kapital allein kein Garant für den Lernerfolg. Coleman zufolge kann das Sozialkapital durch das Vorhandensein vielfältiger unterstützender Beziehungen zwischen Eltern, deren Kinder dieselbe Schule besuchen, das Lernen begünstigen. Bei diesen Arten der Förderung kann es sich um Hausaufgabenhilfe, außerschulische Aktivitäten sowie eine direkte Beteiligung der Eltern an schulischen Aktivitäten und die Unterstützung von Familien und Kindern in Problemsituationen handeln. Empirische Belege für die Wirkungseinflüsse des Sozialkapitals auf die Unterrichtsergebnisse von Schülern auf der Ebene von US-Bundesstaaten finden sich in Putman (2000a) (wegen näherer Einzelheiten vgl. Anhang C).

Bei der Ermittlung der wichtigsten Bestimmungsfaktoren für die schulischen Leistungen ist Vorsicht geboten. Materielle Ressourcen oder hoch qualifizierte Lehrer allein reichen nicht aus. Vieles hängt davon ab, wie die verschiedenen Akteure, d.h. die Schüler, Eltern, Lehrer und die anderen Mitglieder der Schulgemeinschaft im weiteren Sinne, miteinander umgehen und die verfügbaren Ressourcen einsetzen. Die Identifizierung der Schlüsselfaktoren wird dadurch zusätzlich erschwert, dass die Resultate einerseits davon abhängen, was auf der Ergebnisseite gemessen wird (die Effekte unterscheiden sich je nach Fach- oder Kompetenzbereich), und andererseits dadurch beeinflusst werden, welche Bevölkerungsgruppe untersucht wird (eine auf eine bestimmte Gruppe zugeschnittene Förderung ist für diese möglicherweise effizienter als für andere Gruppen). Generell sind engere partnerschaftliche Beziehungen zwischen Schülern, Eltern und Lehrern in einem angemessenen institutionellen und finanziellen Umfeld von wesentlicher Bedeutung.

Die Nachfrage nach berufsspezifischer Fortbildung ist in der Gruppe der Arbeitskräfte jungen bis mittleren Alters wie auch unter Personen mit überdurchschnittlichem Bildungsniveau am größten. Generell hängt die Nachfrage nach Fort- und Weiterbildung mit den Gewohnheiten und Möglichkeiten zusammen, die ein Mensch in früheren Jahren seines Lebens angenommen bzw. geboten bekommen hat (OECD, 1998). Ein Großteil der Erwachsenenbildung 
Soziale Netze tragen zur Förderung des lebensbegleitenden Lernens bei.
Die Verteilung ist von allgemeingültigen Normen und der Qualität des Humankapitals abhängig. ist nicht formell, erfahrungsgebunden und mit dem Alltags- und Arbeitsleben verwoben, so dass sich die Beobachtung und Messung kompliziert gestalten. Die Faktoren, die für die Wirksamkeit und Qualität informeller Lernmethoden sorgen, lassen sich nur schwer beobachten und messen. Die vorhandenen Informationen betreffen eher die formelle bzw. die Fort- und Weiterbildung. Hier deuten viele Hinweise darauf hin, dass im Falle berufsbezogener Fortbildungsmaßnahmen Programme, die den Bedürfnissen einzelner Lernender gerecht werden und am Arbeitsmarkt gefragte Qualifikationen und Kenntnisse vermitteln, am besten in Partnerschaft mit den Lernenden, Programmanbietern und Arbeitgebern entwickelt werden (OECD und US-Bildungsministerium, 1998). Formelle bzw. schulähnliche Unterrichtsmethoden können keine effektiven Resultate garantieren, da der Lernkontext ein anderer ist und es einer stärkeren Anpassung an die spezifischen Bedürfnisse des Einzelnen bedarf. Im Bereich der weniger formellen und weniger berufsspezifischen Lernformen ist hingegen ein sehr viel breiteres Spektrum an Erfahrungen und Konstellationen möglich ${ }^{10}$. Entscheidend ist dabei, dass die Interessen der Erwachsenen und nicht nur ihre Qualifikationsdefizite ermittelt werden.

Auch wenn sich die einzelnen Faktoren nur schwer isolieren lassen, scheint ein hohes Niveau an sozialem Engagement und Vertrauen Lernmöglichkeiten im informellen und formellen Kontext zu eröffnen. Ohsako (1998), der die Ergebnisse des „International Comparison of Learning and Social Participation by the Elderly" (ICLSE - Internationaler Vergleich der Teilnahme älterer Menschen an Weiterbildungsmaßnahmen und sozialen Aktivitäten) ${ }^{11}$ zitiert, berichtet in Bezug auf ältere Bürger über einen Zusammenhang zwischen der Intensität der Teilnahme an Weiterbildungsaktivitäten und ihrer aktiven Beteiligung am Gemeinschafts- und Sozialleben. Ehrenamtliche Vereinigungen können wichtige Orte zur Förderung einer Lernkultur sein. Zwischen dem Niveau der Erstausbildung, Weiterbildung und Fortbildung wie auch dem Grad des Engagements und Vertrauens in gesellschaftliche Aktivitäten scheint es starke Wechselwirkungen zu geben, wodurch ein regelrechter Tugendkreis entsteht. Wie in Kapitel 3 näher erläutert wird, können einige soziale Netze den Lernprozess auch behindern, wenn beispielsweise die Stärke familiärer oder ethnischer Bindungen das Potential für persönliche Weiterentwicklung und Weiterbildung begrenzt ${ }^{12}$.

\subsection{Verteilung des Humankapitals}

Die Verteilung der Qualifikationen und Lernmöglichkeiten für Erwachsene ist zwischen den Ländern höchst unterschiedlich (OECD und Statistics Canada, 2000). Bevölkerungsgruppen, die für Schulversagen anfällig und mithin der Gefahr der Ausgrenzung vom Arbeitsmarkt und von der Gesellschaft ausgesetzt sind, beginnen ihre formelle Bildung gleich mit einem Handicap. Im Laufe der Zeit kann sich dieser Nachteil dann in der Schule verstärken und dazu führen, dass der entsprechende Personenkreis nur sehr niedrige Schulabschlüsse erzielt, nur ein sehr geringer Anteil weiterführende Bildungsangebote nutzt und einen Arbeitsplatz findet. Bildungsnachteile sind auf verschiedenste Art und Weise definiert und gemessen worden, je nachdem ob vorwiegend einzelne Personen oder Gruppen betrachtet wurden oder das Phänomen unter dem Aspekt Finanzierung, Zugang, Bildungsabschlüsse oder Erfolgsquoten bzw. Kompetenzniveaus im Alltag untersucht wurde. Auf Schulebene stellte 


\section{Die Situation der weniger Begünstigten hat sich in vielen Ländern nicht verbessert ...}

eine der verwendeten Definitionen die Situation des Kindes in den Mittelpunkt, „das in der Schule benachteiligt ist, weil es auf Grund wirtschaftlicher, kultureller oder sozialer Faktoren über andere Kompetenzen verfügt als die in der Schule gefragten und zur Erleichterung der Anpassung an die Schule und das Lernen notwendigen“ (Kellaghan, 1999).

In den meisten OECD-Ländern ist der Bevölkerungsanteil der Erwachsenen, die nicht über grundlegende Rechenfertigkeiten sowie Lese- und Schreibkompetenzen verfügen, gesunken, was auf die verzögerten Effekte im Zusammenhang mit der Ausweitung der Schulpflicht in der zweiten Hälfte des 20. Jahrhunderts zurückzuführen ist. Nicht offensichtlich ist hingegen, ob die Bildung zur Schließung der Lücke zwischen den verschiedenen gesellschaftlichen Gruppen beigetragen hat, sei es im Bereich des Zugangs zu Weiterund Hochschulbildung oder in Bezug auf die relative Stellung verschiedener Gruppen am Arbeitsmarkt und in der Gesellschaft. In einigen Fällen dürfte die Expansion der Nachfrage nach Hochschulbildung nicht den sozial Benachteiligten zugute gekommen sein, und sie hat die Arbeitsmarktlage für Geringqualifizierte möglicherweise sogar noch weiter verschlechtert. Belege für diese Hypothese finden sich in OECD (2001). Seit den achtziger Jahren haben sich die Unterschiede der Arbeitseinkommen in einigen OECD-Ländern wie den Vereinigten Staaten vergrößert, und dies nicht nur zwischen den verschiedenen Bildungsniveaus, sondern auch innerhalb derselben. Mögliche Erklärungen hierfür sind u.a. die größeren Qualitätsunterschiede in der Erstausbildung, die sich wandelnde Nachfrage nach verschiedenen Qualifikationsformen, die sich anhand des Niveaus der Erstausbildung nicht messen lassen, wie auch sonstige arbeitsmarkt- oder institutionsspezifische Gründe (Levy und Murnane, 1999).

Eine Studie von Blossfeld und Shavit (1993), in der 13 Länder ${ }^{13}$ und zwei Alterskohorten (1910 und 1960 Geborene) untersucht wurden, hat gezeigt, dass der sozioökonomische Hintergrund in den meisten Ländern nach wie vor ein entscheidender Bestimmungsfaktor für das Bildungsniveau und die Teilnahme an Bildungsaktivitäten war. Dies galt besonders für den Bereich der Hochschulbildung, wo trotz der zunehmenden Zahl der Sekundarschulabschlüsse die Zugangshindernisse (z.B. aus Kosten-, Informations- bzw. Motivationsgründen oder wegen der zuvor erworbenen Fähigkeiten) fortbestehen. Sie fanden ebenfalls heraus, dass die soziale Herkunft auf den niedrigeren Niveaus (beispielsweise beim Übergang von der Sekundarstufe I zur Sekundarstufe II) einen stärkeren Einfluss hat, da die soziale Selektion früher erfolgt. Schließlich bekräftigten die Autoren, dass Bildungsreformen keine oder kaum Auswirkungen auf den Abbau der Ungleichheiten hatten, wenn auch Schweden und die Niederlande hier möglicherweise eine Ausnahme bilden. Demgegenüber fanden Blossfeld und Shavit Belege für eine gewisse Verringerung der Genderunterschiede im Zuge der Bildungsexpansion.

Erikson und Jonsson (1996) heben drei Hauptfaktoren hervor, die dem ungleichen Angebot an Bildungschancen und den unterschiedlichen Bildungsergebnissen in den Ländern zugrunde liegen:

- Unterschiede in den „außerschulischen“ Lernmöglichkeiten,

- Unterschiede in den Überlebensquoten und Möglichkeiten zum Einschlagen ,prestigeträchtiger“ Bildungswege,

- Unterschiede in der globalen Bildungsexpansion. 
Gleichwohl können sorgfältig konzipierte und zielorientierte Programme positive Auswirkungen haben.

\section{Wirtschaftliche Ungleichheit geht mit Ungleichheiten beim Bildungszugang und Bildungsniveau Erwachsener Hand in Hand.}

Erikson und Jonsson verweisen bei der Erklärung der zwischen den Ländern bestehenden Unterschiede insbesondere auf die Rolle des zweiten der drei oben genannten Faktoren. Eine relative sozioökonomische Gleichheit hat demnach im Verein mit einer umfassenden Bildungsreform und Verlängerung der Schulpflicht dazu beigetragen, die Disparitäten im Laufe der Zeit zu reduzieren. Je älter die Schüler sind, wenn sie die Schule verlassen können und zwischen verschiedenen Bildungswegen wählen müssen, desto geringer ist die Gefahr des Versagens.

Obwohl Hanushek und Somers (1999) nur schwache Hinweise darauf finden, dass zwischen den öffentlichen Ausgaben in der Gesamtheit der US-Bundesstaaten, dem Bildungsniveau und letztlich den Arbeitsmarktergebnissen der Schülerinnen und Schüler ein Zusammenhang besteht, legen andere Untersuchungen den Schluss nahe, dass sorgfältig konzipierte und zielgerichtete Programme positive Auswirkungen auf den Wissenserwerb und die Chancen im weiteren Leben haben können, wenn sie den Bedürfnissen der Lernenden aus benachteiligten Verhältnissen gerecht werden (Kellaghan, 1999). So kann die kognitive Entwicklung beispielsweise schon in früher Kindheit in Kinderbetreuungseinrichtungen und in der Schule gefördert werden, wodurch sich die Lernfähigkeiten und Unterrichtsergebnisse auf lange Sicht verbessern (OECD, 1999a). Gleichzeitig können die Effekte von Maßnahmen, die auf benachteiligte Jugendliche zugeschnitten sind, nicht ausschließlich unter dem Gesichtspunkt der kognitiven Ergebnisse beurteilt werden. Andere mögliche Vorteile der Sozialisierung und der persönlichen Entfaltung im Zusammenhang mit diesen Interventionen müssen ebenfalls berücksichtigt werden, auch wenn sie sich schwerer messen lassen.

In der Erwachsenenbevölkerung sind Personen mit geringen Qualifikationen und niedrigem Bildungsniveau stärker der Gefahr der Arbeitslosigkeit und sozialen Ausgrenzung ausgesetzt (Steedman, 1996). Gesellschaften, die in Bezug auf den Bildungszugang und den Lernerfolg weniger auf Gleichbehandlung achten, weisen in der Regel auch eine ungleichmäßigere Einkommensverteilung auf. Bildung, sozialer Hintergrund und Zugang zu Sozialkapital sind Faktoren, die allesamt die Lebenschancen beeinflussen können. Der International Adult Literacy Survey der OECD (Internationale Erhebung über Grad und Verteilung elementarer Grundqualifikationen Erwachsener - OECD und Statistics Canada, 2000) ergibt in diesem Zusammenhang:

- große Unterschiede im globalen Kompetenzniveau (einschließlich Verständnis von Prosa, schematischen und numerischen Darstellungen ${ }^{14}$ ) zwischen den Ländern;

- relativ große Bevölkerungsgruppen mit geringen Qualifikationen, selbst in Ländern mit generell hohem Bildungsniveau;

- starke Verknüpfungen zwischen Kompetenzniveau und dem Erfolg auf beruflicher, gesellschaftlicher und sozialer Ebene.

Länder, in denen die Bevölkerung im erwerbsfähigen Alter ein ausgewogeneres Niveau der Grundkompetenzen aufweist, schneiden auf gesamtwirtschaftlicher Ebene im internationalen Vergleich in der Regel besser ab. Das deutet darauf hin, dass ein Schlüssel zur Anhebung des globalen Kompetenzniveaus und Deckung des künftigen Qualifikationsbedarfs darin bestehen könnte, sich um die Personengruppen zu kümmern, die auf Grund von sozialen und Bildungsungleichheiten nur niedrige Qualifikationen besitzen. 
Willms (2001) zufolge besteht zwischen dem familiären, dem Bildungsund sozialen Hintergrund der Menschen auf der einen und den schulischen Leistungen sowie Kompetenzen Erwachsener auf der anderen Seite ein sehr enger Zusammenhang. Diese Feststellung trifft auf die verschiedenen von ihm untersuchten Länder und für Untergruppen in diesen Ländern zu. So ergibt sich, dass bei einem niedrigen Bildungsniveau oder sozialen Status der Eltern die Unterschiede bei den Lese- und Schreibkompetenzen innerhalb von gesellschaftlichen Untergruppen oder Ländern größer sind als bei einem höheren Bildungsniveau und sozialen Status der Eltern.

Bekräftigt wird diese Feststellung dadurch, dass Länder, denen es gelungen ist, im internationalen Vergleich ein hohes Kompetenzniveau Erwachsener zu erlangen (namentlich die nordischen Länder und die Tschechische Republik), dies zum Teil offenbar durch den Abbau von Ungleichheiten zwischen verschiedenen sozialen Gruppen im Bereich der Lese- und Schreibkompetenzen erreicht haben. Die Vereinigten Staaten zeichnen sich durch ein generell hohes Bildungsniveau aus und liegen bei den Grundqualifikationen der Erwachsenen im Mittelfeld, zählen aber auch zu den OECD-Ländern, in denen unter den unzureichend Ausgebildeten die höchsten Konzentrationen an minder qualifizierten Kräften bestehen. Daher könnte sich eine Verbesserung der elementaren Grundqualifikationen sehr positiv auf die Bevölkerungsgruppen mit niedrigstem Bildungsstand auswirken.

\subsection{Entwicklung der Nachfrage nach Humankapital}

Für Diskrepanzen zwischen Nachfrage und Angebot an Humankapital sind verschiedene Erklärungen denkbar.

Insbesondere im Bereich der Hochschulbildung sind Anzeichen einer gewissen , ̈̈berbildung"vorhanden ...
Zwischen den Bildungsabschlüssen (definiert nach Art oder Grad) bzw. Qualifikationen der Erwerbsbevölkerung und der Nachfrage am Arbeitsmarkt besteht möglicherweise eine große Diskrepanz. Zu einer „Qualifikationsinflation" kommt es, wenn Arbeitgeber das für die Besetzung einer Stelle vorausgesetzte Bildungsniveau systematisch anheben, ohne dass der Posten selbst entsprechende Qualifikationen erfordert.

Der rasche Anstieg des Bildungs- und Kompetenzniveaus in den vergangenen zehn Jahren deutet darauf hin, dass es den OECD-Ländern nicht an Humankapital mangelt. Viele Arbeitsmarktanalytiker, darunter auch Hartog (1997), beobachten sowohl in Europa als auch in den Vereinigten Staaten ein Phänomen, das sie Überbildung nennen, wobei sich dieses Phänomen in Europa im Laufe der Zeit durchaus verstärkt haben könnte. Green, McIntosh und Vignoles (1999) finden Belege für eine gewisse Zunahme der Überbildung im Vereinigten Königreich im Zeitraum zwischen den siebziger und achtziger Jahren des 20. Jahrhunderts, aber nur wenig Anzeichen für eine weit verbreitete „Qualifikationsinflation“. Angesichts des Mangels an Belegen für einen generellen oder langfristigen Rückgang der geschätzten Bildungsrenditen (OECD, 1998) muss die Hypothese der „Überbildung“ mit Vorsicht betrachtet werden. Außerdem ist der Begriff „Überbildung“ insofern etwas irreführend, als er weiterreichende Funktionen der Bildung einfach ignoriert, die außerhalb des Berufslebens eine wichtige Ressource oder Quelle des persönlichen Wohlbefindens schlechthin darstellen.

Arbeitgeber verwenden Bildungsqualifikationen möglicherweise als Signale oder „Auswahlinstrumente“, um natürliche Fähigkeiten, Motivation und Eignungen zu ermitteln, die streng genommen nicht unbedingt Bildungsergebnisse 
... während gleichzeitig Anzeichen für Unterqualifizierung vorhanden sind. sind (Spence, 1973). Mit anderen Worten sind die mit diesen Qualifikationen verbundenen Gehaltsvorteile z.T. jenen schon früher erworbenen Fähigkeiten zu verdanken und nicht etwa dem Mehrwert, der aus dem erworbenen Bildungsgrad resultiert. Man spricht in diesem Zusammenhang von einer „Signalfunktion" (signalling). Es bestehen kaum Zweifel daran, dass dieser Prozess bei der Erklärung der vom jeweiligen Bildungsabschluss abhängigen Gehaltsunterschiede eine gewisse Rolle spielt, seine Gesamtbedeutung bleibt aber dennoch weiterhin kontrovers ${ }^{15}$.

Aus einer Analyse der Disparitäten zwischen offenen Stellen und Qualifikationen geht unter Verwendung von Daten der Internationalen Erhebung über Grad und Verteilung elementarer Grundqualifikationen Erwachsener (IALS) im Vereinigten Königreich (Green, McIntosh und Vignoles, 1999) hervor, dass für einen gegebenen Bildungsgrad nicht nur ein niedrigeres Qualifikationsniveau, sondern auch die ungenügende Nutzung der Kompetenzen sich nachteilig auf das Arbeitseinkommen auswirken. Bei kanadischen Hochschulabsolventen mit schwachem Grundkompetenzniveau ist die Gefahr einer Diskrepanz zwischen Bildungsgrad und Stellenangebot sehr viel größer als bei anderen Hochschulabsolventen (Boothby, 1999).

Der Strukturwandel in der Wirtschaft hat parallel zur zunehmenden Bedeutung der Spitzentechnologiesektoren, mit der eine stetige Anhebung des geforderten Bildungsniveaus in allen Bereichen einhergeht, Veränderungen in der Kompetenznachfrage zur Folge. Levy und Murnane (1999) führen den in den Vereinigten Staaten beobachteten Trend zu Fluktuationen der Arbeitseinkommen (innerhalb der verschiedenen Bildungsabschlussgruppen) z.T. auf die Tatsache zurück, dass so genannte ,soft skills“, wie interpersonelle Kommunikations-, Teamarbeits- und Problemlösungsfähigkeiten, die in expandierenden Dienstleistungs- und Marketingsektoren zunehmend gefragt sind, Gehaltsvorteile mit sich bringen. Diese soft skills spiegeln sich im Niveau der Bildungsabschlüsse nicht wider, führen aber zu Gehaltsunterschieden innerhalb eines bestimmten Bildungsgrads. In der Fachliteratur ist immer häufiger davon die Rede, dass sich das wachsende Lohngefälle in einigen Ländern durch die unterschiedlichen Renditen der verschiedenen Kompetenzen erklären könnte (z.B. Carliner, 1996; Rivera-Batiz, 1994). Diese Studien legen den Schluss nahe, dass sich quantitative Qualifikationen im Vergleich zur Prosa-Lesekompetenz in einigen Ländern stärker auszahlen, namentlich in Kanada, dem Vereinigten Königreich und den Vereinigten Staaten.

Arbeitgeber suchen Arbeitskräfte, die nicht nur besser qualifiziert, sondern auch flexibler und „lernfähiger" sind. Aus diesem Grund erwerben die Arbeitskräfte eine solide Grundausbildung wie auch generelle Beschäftigungsfähigkeiten, um dann auf dieser Grundlage ihre Beschäftigungsmöglichkeiten zu verbessern. Ein intensiverer Bedarf an „Wissensaustausch“ und Organisationskapital auf Organisations- und Unternehmensebene bedeutet, dass nicht nur besser qualifizierte Arbeitskräfte, sondern auch effizientere Managementpraktiken, mehr Teamarbeit und Flexibilität gefragt sind. Die Entwicklung neuer Formen des Organisationskapitals, die mehr Teamarbeit, weniger hierarchische Kontrolle und mehr individuelle Verantwortung voraussetzen, lassen vermuten, dass neue Qualifikationsprofile und Formen der interpersonellen Zusammenarbeit entwickelt werden bzw. entstehen. Daten von zwei 1986 und 
1997 in Großbritannien durchgeführten Erhebungen zeigen, dass in der Einstellungspraxis der Unternehmen in jüngster Zeit Kommunikationsfähigkeiten, „soziale Kompetenzen“ und Problemlösungskapazitäten stärker gefragt waren (Green et al., 1997). In traditionelleren und weniger wissensorientierten Sektoren geht der Trend nun dahin, von Arbeitskräften die Erledigung immer enger definierter Aufgaben zu verlangen, obwohl auch in den traditionellen Sektoren in vielen Unternehmen Teamarbeit und Flexibilität verlangt werden. Bekräftigt wurde diese Feststellung auch andernorts durch Untersuchungen der Arbeitsplatz- und Unternehmensorganisation (beispielsweise Cappelli und Rogovski, 1994; Freeman, Kleiner und Ostrogoff, 1997).

\subsection{Welchen Einfluss hat das Humankapital auf das wirtschaftliche Wohlergehen?}

Das Humankapital hat positiven Einfluss auf Arbeitseinkommen, Beschäftigung und Wirtschaftswachstum.
Zwischen Bildung und Beschäftigung bzw. Arbeitseinkommen besteht ein positiver Zusammenhang.
Eine Möglichkeit, die Wirkung des Humankapitals auf die Wirtschaft zu messen, besteht darin, entweder die Produktivität der Bildungsinvestitionen oder ihre Rendite anhand der Arbeitseinkommen zu berechnen. Private Renditen lassen sich anhand der auf die Lebenszeit bezogenen privaten Ausgaben und Arbeitsverdienste nach Steuern schätzen (vgl. OECD, 1998). Grundsätzlich sollten diese privaten Renditen auch die nicht monetären Vorteile berücksichtigen, wie die Freude am Lernen und die größere Berufszufriedenheit, die ein Qualifikationsnachweis verleihen kann. Soziale Renditen sollten das gesamte Spektrum der durch zusätzliche Humankapitalinvestitionen bedingten Vor- und Nachteile für Öffentlichkeit und Gesellschaft umfassen. In der Praxis stößt man bei der Berechnung der Gesamtkosten und -renditen aber auf große Schwierigkeiten, und die veröffentlichten Schätzungen basieren häufig auf einem verhältnismäßig engen Spektrum messbarer Faktoren. So ist es insbesondere schwierig, dem Effekt von Fortbildungsmaßnahmen am Arbeitsplatz auf das Gehaltsniveau bzw. den Nutzeffekten des Humankapitals, die der Gesellschaft insgesamt zugute kommen, oder auch vielen nicht ökonomischen Vorteilen voll Rechnung zu tragen. Ein alternatives Konzept besteht darin, auf der Grundlage nationaler oder regionaler Statistiken empirische Belege für den Einfluss des Humankapitalbestands und dessen Entwicklung auf Niveau und Zuwachsrate des Wirtschaftswachstums zu suchen. In zahlreichen Arbeiten werden derartige Schätzungen unter Verwendung sowohl mikro- als auch makroökonomischer Daten vorgenommen.

\subsubsection{Mikroökonomische Daten zu Arbeitseinkommen und Beschäftigung}

Bei höher gebildeten Personen ist die Wahrscheinlichkeit einer Beschäftigung größer, und sofern sie wirtschaftlich aktiv sind, ist die Gefahr der Arbeitslosigkeit geringer. Höhere Bildungsabschlüsse bieten ferner Gehaltsvorteile. In einigen Ländern sind diese Vorteile sehr hoch und schlagen sich in einer größeren Bandbreite der Einkommen am Arbeitsmarkt und unter Umständen auch einer stärkeren Rendite spezifischer Qualifikationen nieder. Mikroökonomische Belege zeigen, dass sich ein zusätzliches Bildungsjahr allein im Schnitt in einem Gehaltszuwachs zwischen 5\% und 15\% niederschlägt (Krueger und Lindahl, 1999). 
Unabhängig von den Qualifikationen haben die Grundkompetenzen Erwachsener starke Auswirkungen auf ihre Arbeitsverdienste.

Die Rolle des Humankapitals für das Wirtschaftswachstum lässt sich nur schwer erklären.

Der Wissenstransfer ist von entscheidender Bedeutung ...
In einem verbundenen Forschungsbereich wird der Einfluss verschiedener Grundkompetenzen Erwachsener auf die Arbeitsverdienste untersucht. Belege aus den Vereinigten Staaten deuten darauf hin, dass die ökonomische Rendite von Kompetenzen mit der Wissensintensität des Arbeitsplatzes zunimmt (Raudenbush und Kasim, 1998). In einer britischen Längsschnittstudie stellten Bynner et al. (2001) für Niveau-I-Rechenfertigkeiten gemäß der UK Qualification and Curriculum Authority (QCA) ${ }^{16}$ Renditen von 8-10\% fest. Daten der Internationalen Erhebung über Grad und Verteilung elementarer Grundqualifikationen Erwachsener (OECD und Statistics Canada, 2000) zeigen, dass Bildung, Lese- und Grundkompetenzen, Erfahrung, Geschlecht, Bildungsniveau der Eltern sowie Gebrauch der Muttersprache - Faktoren, die sich allesamt auf das Humankapital beziehen - für rd. 20-50\% der Verdienstschwankungen am Arbeitsmarkt insgesamt verantwortlich sind (Abb. 4.10, S. 76). Die Korrelation zwischen Grundkompetenzen und Bildungsabschluss auf der einen und Arbeitsverdiensten auf der anderen Seite ist von Land zu Land verschieden, so dass auch die nationalen und institutionellen Effekte auf die Art und Weise, wie die Beherrschung der Grundkompetenzen an den nationalen Arbeitsmärkten honoriert wird, voneinander abweichen.

\subsubsection{Makroökonomische Daten zum BIP-Wachstum}

Ein auf den Faktoren Sachkapital und Arbeit basierendes Standardmodell des Wirtschaftswachstums wurde von Solow entwickelt (1956). In diesem Modell wächst die gesamtwirtschaftliche Produktion rascher als die zwei wichtigsten wirtschaftlichen Inputfaktoren - Kapital und Arbeit -, so dass eine „Restgröße“ übrig bleibt, die den technischen Fortschritt widerspiegelt. Kritiker derartiger empirischer Wachstumsratenanalysen machen geltend, dass Messungen der Qualität des Humankapitalbestands problematisch seien. Wie Barro und Sala-i-Martin (1995) überdies feststellten, liefern die Ergebnisse der empirischen Wachstumsratenanalysen keine Erklärung dafür, dass die beobachtete Realität so stark von den Erwartungen abweicht ${ }^{17}$.

Eine Alternativlösung zur Messung der Effekte verschiedener Faktoren auf das BIP-Wachstum besteht darin, länderübergreifende Regressionsanalysen zu verwenden, die Variablen für Sachkapital, Bildung, Einkommensniveau und in einigen Fällen auch Ersatzvariablen für verschiedene soziale und institutionelle Faktoren enthalten (Barro, 2001). Allerdings können die Auswahl der Länder, die berücksichtigten Variablen, die Wahl der Zeitperioden wie auch die Spezifizierung der Modelle ${ }^{18} \mathrm{zu}$ erheblichen Ergebnisunterschieden führen. Einige Untersuchungen beziehen sich auf Industrie- und Entwicklungsländer zugleich. Die Statistiken gewinnen wegen des dadurch bedingten breiteren Spektrums an Wachstumsbestimmungsfaktoren an Aussagekraft. Gleichzeitig wird implizit unterstellt, dass Industrie- und Entwicklungsländer einige Bestimmungsfaktoren des Wachstums gemeinsam haben - auch wenn diese quantitativ voneinander abweichen.

In frühen Modellen des Wirtschaftswachstums spielten die unterschiedlichen Bildungs- und Qualifikationsniveaus keine explizite Rolle, und dem Potential des Humankapitals zur Schaffung von „Externalitäten“ oder „Übergreifeffekten“ (so z.B. durch dessen Einfluss auf die Produktivität anderer Faktoren) wurde keine Aufmerksamkeit geschenkt. Mit der Entwicklung der so genannten „neuen Wachstumsmodelle“ (Lucas, 1988; Romer, 1990b; Barro und

(C) OECD 2004 
... insbesondere in der Beziehung zwischen Hochschulausbildung und angewandter Forschung und Entwicklung.
Sala-i-Martin, 1995) wurde die Rolle von Bildung und Lernen bei der Produktion neuer Technologien und Innovationen stärker in den Mittelpunkt gerückt. Neue Konzepte und Ideen, die in der Forschung und Entwicklung sowie in wissensintensiven Sektoren entstanden sind, verstärken die Produktivität von Sachkapitalinvestitionen in anderen Sektoren und Regionen. Der von innen angetriebene technische Wandel, die zunehmenden Skaleneffekte und das bei der technologieintensiven Produktion erworbene Know-how können dem Wachstum der gesamtwirtschaftlichen Produktion Impulse verleihen. So kann ein expandierender, exportorientierter Sektor von Spitzenformat in der gesamten Volkswirtschaft Wissen und Innovationen Impulse verleihen, indem Kompetenzen und Unternehmer mobilisiert sowie neue Technologien und Produkte verbreitet werden. Der ursprüngliche Bestand an Humankapital in einer früheren Periode kann Innovationen und Downstream-Effekte in Form von Übergreifeffekten oder positiven Externalitäten auslösen, die andere Unternehmen, ja sogar ganze Regionen oder Länder beeinflussen (Acemoglu, 1996). Ein Teil des ursprünglichen Humankapitalbestands geht auf grundlegende oder angewandte wissenschaftliche Kenntnisse zurück, die im Rahmen der Hochschulausbildung erworben wurden. Harberger (1998) unterscheidet zwischen den so genannten „Hefe“- und „Pilz“-Effekten auf die Restgröße in Wirtschaftswachstumsmodellen. Dabei sind Wissen und Humankapital quasi die Hefe, mit der die Produktivität überall in der Volkswirtschaft auf verhältnismäßig gleichförmige Art und Weise gesteigert wird, während andere Faktoren wie technologische Durchbrüche oder Entdeckungen plötzlich wie Pilze aus der Erde schießen und die Produktivität in einigen Sektoren wesentlich stärker steigern als in anderen.

Die Hochschulausbildung spielt bei der Entwicklung der innovativen Forschung und beim Erwerb des notwendigen Wissens für die Integration dieser Forschung eine entscheidende Rolle. So haben einige „neue Wachstumstheorien“" versucht, ein komplexeres Modell zu erstellen, in dem bei der Humankapitalbildung nicht nur der Bildung selbst, sondern auch ihren Nebenprodukten, wie Forschung und Innovation, größte Bedeutung beigemessen wurde. Werden beispielsweise die FuE-Ausgaben in Wachstumsmodelle einbezogen, dann fällt der Effekt der Bildung für sich genommen offenbar geringer aus. Unter Verwendung des BIP-Anteils der FuE-Ausgaben als Näherungsgröße für technologisches Know-how kommen Nonneman und Vanhoudt (1996) zu dem Ergebnis, dass ein Teil des Wachstums, das der Erstausbildung zugeschrieben wurde, eigentlich den FuE-Ausgaben zu verdanken war.

Untersuchungen der Bildungseffekte auf das Wirtschaftswachstum waren oft wenig aufschlussreich. Zurückzuführen ist dies möglicherweise z.T. auf die mangelhafte Datenqualität und z.T. auf die Schwierigkeit, die komplexen Interaktionen zu identifizieren, durch die das Humankapital im Wachstumsprozess Bedeutung erlangt. Pritchett (1999) ist der Auffassung, dass höhere Schulbesuchsquoten bzw. Bildungsabschlüsse keine signifikant positiven Auswirkungen auf die Steigerungsrate der Produktivität oder das Wirtschaftswachstum hatten ${ }^{19} . \mathrm{Zu}$ den am häufigsten für das Humankapital verwendeten Ersatzmessgrößen zählen: Bruttoschulbesuchsquoten (z.B. der prozentuale Anteil der Schüler in der Sekundarstufe); durchschnittliche Schulbesuchsdauer der Erwachsenenbevölkerung; Schätzungen des Anteils der Erwerbs- oder Erwachsenenbevölkerung, der eine Primar-, Sekundar- oder Hochschulausbildung absolviert hat, bzw. Schätzungen der Unterrichtsqualität unter Zugrundelegung 
von Schülertestergebnissen oder auf der Basis von Resultaten der Erhebungen über die Grundqualifikationen Erwachsener. Bei der Analyse der Wirkungseinflüsse dieser Ersatzvariablen auf das Wirtschaftswachstum stößt man auf zahlreiche Messprobleme wie auch analytische Herausforderungen, die nur schwer zu bewältigen sind.

a) Manchmal besteht Unklarheit in Bezug auf die Richtung des Kausalzusammenhangs;

b) die Rolle des Humankapitals kann durch seine Interaktion mit anderen Faktoren verschleiert werden: Anpassung an neue Technologien und Arbeitsformen sowie effizientere Allokation von Sachkapital;

c) das Niveau des Bildungsabschlusses ist eine grobe Ersatzvariable für die Rolle von Wissen und Qualifikationen;

d) Messfehler auf Grund einer gemäß internationalen Standards ungeeigneten bzw. nicht vergleichbaren Klassifikation der Bildungsabschlüsse;

e) atypische oder ,aus dem Rahmen fallende“ Länder können die Ergebnisse verzerren (Temple, 2001).

Der unter $b$ ) genannte Fall tritt ein, wenn in den entsprechenden Modellen nicht veranschaulicht werden kann, auf welche Art und Weise einige Länder, die zu Beginn der sechziger Jahre über einen geringen Ausgangsbestand an Humankapital verfügten, vielleicht mehr Möglichkeiten und Anreize zur Wachstumsbeschleunigung hatten, indem sie im Ausland entwickelte Technologien importierten und anwendeten. Andererseits können Länder mit niedrigem ursprünglichen Einkommensniveau, aber hohem anfänglichen Humankapitalbestand (oder einer kritischen Masse an Hochschulabsolventen) von einem Aufhol- oder Konvergenzprozess profitieren, indem sie importierte Technologien übernehmen und anwenden.

Steedman (1996) liefert ein Beispiel für den unter $d$ ) genannten Fall und weist auf Inkohärenzen in der Darstellung und Klassifikation der Daten zum Bildungsniveau in der International Standard Classification of Education (ISCED - Internationale Standardklassifikation des Bildungswesens) hin. Krueger und Lindahl (1999) vertreten ebenfalls die Auffassung, dass diese Ergebnisse auf Messfehler in den zugrunde gelegten, wichtigsten internationalen Datenquellen zurückzuführen sind, vor allem die negativen Ergebnisse hinsichtlich des Niveaus der Bildungsabschlüsse von Frauen ${ }^{20}$. Das letztgenannte Ergebnis ist schwer mit der Auffassung zu vereinbaren, der zufolge die Bildung von Mädchen und Frauen einen wichtigen Beitrag zu Wirtschaftswachstum und Wohlstand in den Industrie- wie in den Entwicklungsländern leistet ${ }^{21}$.

Indikatoren des Humankapitals, die auf einer direkten Kompetenzmessung basieren, zeigen positivere Ergebnisse ...
Ein Nachteil der meisten länderübergreifenden Arbeiten ist die Wahrscheinlichkeit bedeutender Unterschiede in Natur und Qualität des Schulwesens zwischen den Ländern, die die Nützlichkeit internationaler Vergleiche in Frage stellen könnten (Temple, 2001). Hanushek und Kimko (2000) sowie Barro (2001) schätzen unter Verwendung von Daten internationaler Tests kognitiver Fähigkeiten in den Fächern Mathematik und Naturwissenschaften die Bildungsqualität verschiedener Gruppen der Erwachsenenerwerbsbevölkerung. Sie kamen zu dem Ergebnis, dass Messgrößen, die auf der Bildungsqualität basieren, die Bildungseffekte des Wirtschaftswachstums in den einzelnen Ländern sehr viel besser erklären als die einfache Zahl der Studienjahre. 


\section{... und die jüngsten empirischen Arbeiten haben verlässliche Schätzungen der Auswirkungen des Humankapitals auf das Wirtschaftswachstum geliefert.}

Die Hochschulbildung spielt in den OECDLändern eine besonders wichtige Rolle.
Jüngere Arbeiten von de la Fuente und Domenech (2000) sowie Bassanini und Scarpetta (2001) liefern weitere Gründe dafür, dass Messfehler hier eine wichtige Rolle spielen. De la Fuente und Domenech (2000) haben den BarroLee-Datensatz ${ }^{22}$ wie auch andere Datenquellen untersucht, u.a. die von Nehru, Swanson und Dubey (1995). Bei der Untersuchung der intertemporalen Konsistenz dieses Datensatzes und beim Vergleich der Daten mit neueren, der OECDDatenbank von Bildungsindikatoren entnommenen Daten stießen sie auf erhebliche Messprobleme. Sie haben den Datensatz dann anhand der verfügbaren nationalen und internationalen Quellen verbessert und dabei besonders darauf geachtet, sowohl die Kohärenz im Zeitablauf als auch die Übereinstimmung mit jüngsten, von der OECD gesammelten Daten über Bildungsabschlüsse zu gewährleisten. Unter Verwendung dieses Datensatzes kommen die Autoren zu der Schlussfolgerung, dass das Humankapital in der Tat einen erheblichen und positiven Einfluss auf das BIP-Wachstum bzw. das Pro-Kopf-Einkommen hat. Die Ergebnisse sind insofern bemerkenswert, als sie sich auf einen begrenzten Stichprobenumfang von Ländern beziehen (vor allem OECD-Mitgliedsländer), während in den meisten anderen Studien signifikante Ergebnisse für einen Stichprobenumfang erzielt wurden, der sich aus Hoch- und Niedrigeinkommensländern zusammensetzt. In den Fällen, wo sich die Analysen früherer Untersuchungen ausschließlich auf OECD-Länder bezogen, ergaben sich häufig keine signifikanten Humankapitaleffekte auf das Wachstum (z.B. Barro, 2001).

Die OECD hat für weitere Arbeiten über jüngste Disparitäten beim Wirtschaftswachstum die von de la Fuente und Domenech erstellten Daten verwendet. Aus diesen Arbeiten der OECD geht hervor, dass ,die Verbesserung des Humankapitals einer der Schlüsselfaktoren des Wachstumsprozesses der vergangenen Jahrzehnte in allen OECD-Ländern war, insbesondere aber in Deutschland (vor allem in den achtziger Jahren), Italien, Griechenland, den Niederlanden (vor allem in den achtziger Jahren) sowie Spanien, wo die Verbesserung des Humankapitals im Vergleich zum vergangenen Jahrzehnt für eine Wachstumsbeschleunigung von mehr als $1 / 2$ Prozentpunkt verantwortlich war" (OECD, 2000b). Für die OECD-Länder insgesamt bedeutet dies, dass jedes weitere Jahr Vollzeitausbildung (was einer Erhöhung des Humankapitals um rd. 10\% entspricht) mit einer Steigerung der Pro-Kopf-Produktion von rd. 6\% einhergeht. (Diese Schätzung basiert auf der von uns gewählten Spezifikation. Alternative Spezifikationen deuten auf Werte zwischen 4\% und 7\% hin.)

In dem Maße, wie die meisten OECD-Länder bis zum Ende der Schulpflicht oder sogar der höheren Sekundarschulbildung ein System des universellen Schulbesuchs eingerichtet haben, ist das Interesse an der wachstumsfördernden Rolle der Hochschulbildung bzw. anderer, sich an die Pflichtschule anschließenden Bildungsformen sehr groß. Die Belege sind insofern nicht sehr überzeugend, da generell keine aussagekräftigen Zeitreihendaten verfügbar sind. Gemmell (1996) untersucht aber in einer Studie unter Verwendung eines Index des Bildungsstands der Erwerbsbevölkerung - Anzahl der Arbeitskräfte, die eine Primar-, Sekundar- und Hochschulausbildung absolviert haben - die Effekte der drei Bildungsniveaus in Entwicklungs- und OECD-Ländern im Zeitraum 1960-1985. Nimmt man eine Aufteilung der Länderstichproben nach Einkommensniveau vor, dann ergibt sich unter sonst gleichen Bedingungen, dass die Hochschulbildung für das Wirtschaftswachstum in OECD-Ländern von größerer Bedeutung zu sein scheint, während die Primar- und Sekundarbildung für das Wirtschaftswachstum in Entwicklungsländern eine wesentlichere 
Auch der Studiengang ist in der Hochschulbildung von Bedeutung.

\section{Das Humankapital spielt bei der Einkommens- gleichheit eine Rolle.}

\author{
Insgesamt ergeben \\ sich aus jüngsten \\ Forschungsergebnissen \\ positive Human- \\ kapitaleffekte auf das \\ Wirtschaftswachstum.
}

Rolle spielt. Unter Verwendung von Schulbesuchsdaten erzielte Gemmell (1995) ähnliche Ergebnisse, während Barro und Sala-i-Martin (1995) zu ähnlichen Resultaten unter Zugrundelegung des Bildungsniveaus kamen ${ }^{23}$. Zwischen dem Niveau der Erstausbildung wie auch dem späteren Ausbau der Hochschulbildung und dem Wachstum des Pro-Kopf-Einkommens in den OECD-Ländern wurde ein positiver und signifikanter Zusammenhang festgestellt.

Die Tatsache, dass die Hochschulbildung potentiell erhebliche Übergreifeffekte auf die Produktivität hat, erklärt möglicherweise die Ergebnisunterschiede in den mikro- und makroökonomischen Einkommensstudien. Im ersteren Bereich ist die Rendite des Hochschulabschlusses im Vergleich zu der eines Sekundarstufe-II-Abschlusses Schätzungen zufolge offenbar moderat (OECD, 1998). Auf makroökonomischer Ebene lässt sich die Rolle der Hochschulbildung bei der Schaffung indirekter und übergreifender Effekte besser erfassen, was sich dann auch sofort in signifikanteren Effekten niederschlägt.

Bei der Evaluierung der Effekte der Hochschulbildung auf die Produktivität muss zwei weiteren Faktoren Rechnung getragen werden, und zwar zum einen dem jeweiligen Anteil der einzelnen Studiengänge am Gesamteffekt sowie der Zahl der Hochschulabsolventen, die in Wirtschaftszweige eintreten, deren BIP-Beitrag nicht präzise gemessen wird (wie beispielsweise die öffentliche Verwaltung oder Dienstleistungssektoren). In einer Untersuchung der Humankapitaleffekte auf das Wachstum der Arbeitsproduktivität in OECDLändern für den Zeitraum 1950-1988 kamen Gittleman und Wolff (1995) zu der Schlussfolgerung, dass die Zahl der Wissenschaftler und Ingenieure je Einwohner signifikant positive Auswirkungen auf die Produktivität hat.

Das Humankapital beeinflusst auch die Strukturen der Einkommensungleichheiten. Die Einkommensungleichheiten, die seit Mitte der achtziger Jahre in vielen OECD-Ländern im Trend zugenommen haben, sind auf vielerlei Faktoren zurückzuführen. Zunehmende Ungleichheiten zwischen Haushalten je nach Beschäftigungsform (Teilzeit, vorübergehende Beschäftigung usw.) sind mit dem Anstieg des Anteils der reichen und armen Erwerbstätigenhaushalte in Verbindung gebracht worden (OECD und Statistics Canada, 2000). Alesina und Rodrik (1992) weisen einen Zusammenhang zwischen Bildung und Einkommensverteilung nach, wobei die Kausalbeziehung in beiden Richtungen besteht. Daten der Internationalen Erhebung über Grad und Verteilung elementarer Grundqualifikationen Erwachsener (OECD und Statistics Canada, 2000) deuten auf eine starke Korrelation zwischen den Einkommensungleichheiten auf nationaler Ebene und Ungleichheiten in der Verteilung der Lesekompetenz (Verständnis von Prosatexten) hin, was den Schluss nahe legt, dass die Einkommensgleichheit größer ist, je gleichmäßiger sich das Humankapital verteilt.

Eine Untersuchung von Temple (2001) zeichnet ein generell positives Bild der Humankapitaleffekte und kommt zu der Schlussfolgerung:

„Im Verlauf der vergangenen zehn Jahre sind die Wachstumsforscher, die zunächst recht spektakuläre Bildungseffekte festgestellt hatten, dazu übergegangen, die Existenz derartiger Effekte ganz und gar in Frage zu stellen. Die Ergebnisse jüngerer Forschungsarbeiten liegen irgendwo zwischen diesen beiden Extremen, tendieren aber eher zu den ursprünglichen Erkenntnissen, denen zufolge die Bildung einen starken Einfluss hat. Eine Untersuchung der Studien, die überhaupt keine Effekte ermittelt haben, 
Die positiven sozialen Effekte der Bildung überwiegen die negativen ...
... und die über die sozialen Nutzeffekte wirksam werdenden indirekten Bildungseffekte auf das Wirtschaftswachstum können genauso stark sein wie die direkten Effekte. liefert überzeugende Gründe (Messfehler, Heranziehung atypischer Länder sowie inkorrekte Spezifikationen) dafür, diese Ergebnisse anzuzweifeln. Die Bilanz der neueren Daten deutet darauf hin, dass die Bildung Auswirkungen auf die Produktivität hat, die zumindest genauso gro $\beta$ sind wie die Effekte, die von Arbeitsökonomen ermittelt wurden. “

\subsection{Welchen Einfluss hat das Humankapital auf die verschiedenen Aspekte des menschlichen Wohlergehens?}

Es ist eine Reihe von Methoden zur Messung der „,sozialen Nutzeffekte“ von Bildung und Lernen entwickelt worden, die weiter unten beschrieben werden. Die Informationen, die in diesem Abschnitt untersucht werden, beziehen sich hauptsächlich auf die gemessenen Auswirkungen der Erstausbildung in Bereichen wie Gesundheit, Sozialversicherung, Elternschaft, Kriminalität sowie persönliches bzw. subjektives Wohlergehen. Einige Effekte des Lernens auf den Gesundheitszustand und das Sozialverhalten können durch die Förderung bestimmter Gewohnheiten, Eigenschaften oder Einstellungen verstärkt werden, die der Arbeitsplatzsuche, der Produktivität, dem persönlichen Wohlbefinden, der positiven Zeiteinteilung wie auch der Selbstdisziplin Vorschub leisten. Einige dieser Merkmale wie auch angeborene Fertigkeiten und Eigenschaften werden außerhalb der formellen Bildung erworben, korrelieren aber sehr eng mit der Ausbildung.

Humankompetenzen können für gesellschaftlich destruktive Zwecke verwendet werden, wie beispielsweise Kriminalität. Bestrebungen um bestimmte Formen des Humankapitals können die Verwirklichung anderer wünschenswerter Ziele ausschließen und die sozialen Beziehungen wie auch das persönliche Wohlbefinden gefährden. Beispielsweise ist eine übermäßig starke Konzentration auf kognitive Fähigkeiten in den frühen Jahren der Kindheit auf Kosten der Sozialisierung und des Spielens wahrscheinlich nicht die Methode, die den Entwicklungsbedürfnissen von Kindern am ehesten gerecht wird. Wie beim Sozialkapital stellen diese potentiellen negativen Auswirkungen aber eher eine Ausnahme dar.

Es ist eine Reihe wichtiger Messtechniken entwickelt worden, um bestimmte soziale Nutzeffekte der Bildung, namentlich die Gesundheit, in Geldwerten auszudrücken. Arbeiten von Wolfe und Haveman (2001), McMahon $(2001)^{24}$ sowie Wolfe und Zuvekas (1997) liefern ein breites Spektrum an Techniken, mit denen sich der monetäre Wert der Bildung schätzen lässt. Diese Techniken beruhen auf Berechnungen der Kosten für den Erwerb derselben Effekte auf anderem Wege ${ }^{25}$. Behrman und Stacey (1997) fassen die Arbeiten aus einer Vielzahl von Quellen weiterreichender sozialer Effekte zusammen, wobei sie hauptsächlich Daten aus den Vereinigten Staaten zugrunde legen. Weitere Belege für die weiterreichenden sozialen Nutzeffekte der Bildung sind jüngsten Berichten im Vereinigten Königreich zu entnehmen ${ }^{26}$.

Unter Verwendung von Kontrollvariablen für Einkommen, ethnische Zugehörigkeit, sozialen Status und andere Faktoren haben Forschungsarbeiten ergeben, dass zwischen einem besseren Gesundheitszustand, einer niedrigeren Kriminalität, der Teilhabe am politischen und gesellschaftlichen Leben wie auch sozialem Zusammenhalt und Bildung eine Korrelation besteht. Dennoch 
Höher gebildete Personen erfreuen sich in der Regel einer besseren Gesundheit ...

\author{
... greifen weniger \\ auf Sozialleistungen \\ zurück ...
}

\author{
... und übertragen \\ einige der Bildungs- \\ vorteile auf ihre \\ Kinder.
}

ist bei der Interpretation dieser Ergebnisse Vorsicht geboten, da die Richtung der Kausaleffekte unklar ist und andere Faktoren eine Rolle spielen können, denen bisher nicht Rechnung getragen wurde. Dennoch kommen Wolfe und Haveman (2001) wie auch McMahon (2001) zu der Schlussfolgerung, dass die sozialen Nutzeffekte der Bildung groß sind - möglicherweise größer als die direkten makroökonomischen und Arbeitsmarkteffekte.

Einer der deutlichsten Nutzeffekte von Bildung ist ein besserer Gesundheitszustand. Personen mit höherem Bildungsabschluss haben einen gesünderen Lebensstil. Bei höher gebildeten Personen ist die Gefahr, dass sie rauchen oder viel trinken, eher geringer. Schätzungen zufolge reduziert jedes zusätzliche Bildungsjahr den tagesdurchschnittlichen Zigarettenkonsum für Männer um 1,6 und für Frauen um 1,1 (Wolfe und Haveman, 2001). Gebildetere Personen sind generell auch weniger oft übergewichtig und treiben mehr Sport als weniger gebildete Menschen - rd. 17 Minuten für jedes zusätzliche Bildungsjahr (Kenkel, 1991). Die mit der Bildung verbundenen gesundheitlichen Nutzeffekte sind z.T. auch durch die Berufs- (Auswahl von Berufen mit verhältnismäßig geringen Gesundheitsrisiken) oder Wohnortwahl (Gebiete mit geringerer Umweltbelastung) bedingt. Gebildetere Personen sind generell auch eher in der Lage, die für ihre Gesundheit relevanten Informationen zu ermitteln, diese zu nutzen und sich entsprechend gesünder zu verhalten (Kenken, 1991). Gleichzeitig veranschaulicht Kenkel aber auch unter Verwendung von US-Daten, dass sich die Gesundheitsunterschiede in den meisten Fällen nicht einfach dadurch erklären lassen, dass der Einzelne mehr oder minder gut über Gesundheitsfragen Bescheid weiß. So übt die Bildung offenbar unabhängig von Einkommen, ethnischer Zugehörigkeit, sozialem Hintergrund und sonstigen Faktoren Einfluss auf die Gesundheit aus.

Ein höheres Bildungsniveau geht generell mit einer geringeren Wahrscheinlichkeit der Inanspruchnahme von Sozialleistungen einher (Wolfe und Haveman, 2001). Neuere Analysen kamen zu dem Ergebnis, dass bei einem höheren Bildungsniveau der Mutter die Wahrscheinlichkeit geringer ist, dass ihre Töchter Sozialhilfe in Anspruch nehmen, wenn diese ihnen zusteht. Untersuchungen bei Antragstellern von Erwerbsunfähigkeitsleistungen ergeben ferner, dass die Wahrscheinlichkeit, diese Form der Unterstützung zu erhalten, mit steigendem Bildungsniveau abnimmt. Höher gebildete Arbeitskräfte weisen generell auch niedrigere Arbeitslosenquoten auf, und sie empfangen höhere Löhne und Gehälter, was der Gesellschaft dann in Form höherer Steuereinnahmen zugute kommt ${ }^{27}$.

Das Bildungsniveau einer Generation hat positive Auswirkungen auf die Bildungsabschlüsse der nachfolgenden Generation. Kinder von Eltern mit Abitur machen mit größerer Wahrscheinlichkeit selbst Abitur als Kinder weniger gebildeter Eltern (Sandefur, McLanahan und Wojtkiewicz, 1989). Kinder von Eltern mit höherem Bildungsabschluss sind in der kognitiven Entwicklung generell weiter fortgeschritten und verfügen auch über ein höheres künftiges Gehaltspotential. Außerdem sind auf der Ebene des sozialen Umfelds in dem Maße Übergreifeffekte beobachtet worden, wie das Leben in einem gesellschaftlichen Kontext mit recht hohem Bildungsniveau die Wahrscheinlichkeit erhöht, dass die Kinder, die in diesem Umfeld leben, unter Annahme sonst gleicher Bedingungen selbst die Sekundarschule abschließen (Wolfe und Haveman, 2001). 
Die Bildung verbessert die Arbeitsplatzsuche und schafft effizientere Verbraucher...

... und wirkt sich auf das bürgerliche Engagement aus.

Auch zwischen Bildung und rückläufiger Kriminalität besteht ein Zusammenhang ...

... wie auch zwischen Bildung und ehrenamtlichen Tätigkeiten bzw. Spenden.
Die Bildung übt einen positiven Einfluss auf die Arbeitsuche aus - wahrscheinlich, weil gebildete Menschen in der Lage sind, Informationen sinnvoll $\mathrm{zu}$ verwerten, sich über Netzwerke Zugang zu Informationen zu verschaffen und wichtige Beziehungen zu knüpfen. Gebildetere Personen sind offenbar oft auch effizientere Verbraucher (Rizzo und Zeckhauser, 1992).

Das Niveau der Bildungsabschlüsse ist einer der wichtigsten Bestimmungsfaktoren für viele Formen des politischen und gesellschaftlichen Engagements. Verba, Schlozman und Brady (1995) kamen zu dem Ergebnis, dass die Bildung unter Annahme sonst gleicher Bedingungen das politische Engagement erhöht. Außerdem besteht zwischen dem Grundbildungsniveau Erwachsener und ihrer Teilnahme an ehrenamtlichen gemeinschaftlichen Aktivitäten in mehreren OECD-Ländern ein positiver Zusammenhang (OECD und Statistics Canada, 2000). Laut Bynner et al. (2001) sind die „sozialen Kompetenzen“ unter Zugrundelegung von Daten für das Vereinigte Königreich bei höherem Bildungsniveau umso stärker. Hierzu zählen Organisationstalent und Beratungsfähigkeiten - d.h. Eigenschaften, die allesamt die Qualität des bürgerlichen Engagements potentiell erhöhen. Ferner beobachten sie eine größere Toleranz gegenüber gesellschaftlicher Vielfalt, Engagement für das Ziel der Chancengleichheit sowie Widerstand gegen jede Form der politischen Resignation.

Einige Ergebnisse sind wiederum nicht so eindeutig. Helliwell und Putnam (1999b) stellten fest, dass ein höheres durchschnittliches Bildungsniveau zu einem Anstieg des Vertrauens in die Gesellschaft, nicht aber einem politischen Desengagement führt, während Nie, Junn und Stehlik-Barry (1996) die Auffassung vertreten, dass das relative und nicht das absolute Bildungsniveau den wesentlichen Bestimmungsfaktor des gesellschaftlichen Engagements darstellen.

Die Bildung reduziert das Kriminalitätsrisiko insofern, als sie junge Menschen, die im Schulverband bleiben, bei der Sozialisierung unterstützt. Wolfe und Haveman (2001) stellen fest, dass die Humankapitalvariablen des Umfelds positive Auswirkungen auf antisoziales Verhalten haben. Auch wenn die Gesamtauswirkungen dieser sozialen Nutzeffekte erst nach einer gewissen Zeit voll zum Tragen kommen, kann die Gesellschaft von Bildungsinvestitionen profitieren, indem sie weniger für Sozialleistungsprogramme sowie Kriminalitätsvorbeugung/Rechtsdurchsetzung ausgibt.

Außerdem gibt es Belege für eine positive Korrelation zwischen dem in ehrenamtliche Tätigkeiten investierten Aufwand an Zeit und Geld und der Zahl der Bildungsjahre. Beispielsweise geht aus einer Studie hervor, dass CollegeAbsolventen im Vergleich zu Sekundarschulabsolventen nahezu doppelt so viel Zeit aufwenden und für Spenden im Verhältnis zu ihrem Einkommen 50\% mehr geben (Hodgkinson und Weitzman, 1988). Im Vereinigten Königreich geht aus Daten der National Child Development Study (NCDS - Nationale Untersuchung der kindlichen Entwicklung) hervor, dass zwischen dem Bildungsniveau und der Mitgliedschaft in politischen Organisationen, Umweltoder Frauengruppen bzw. Wohltätigkeitsverbänden, Vereinen sowie Elternbeiräten eine sehr starke Korrelation besteht (Schuller et al., 2001). In Bynner et al. (2001) wird festgestellt, dass die Wahrscheinlichkeit bei Hochschulabsolventen im Vereinigten Königreich aktives Mitglied einer ehrenamtlichen Organisation zu sein, dreimal so groß ist wie bei Schülern ohne Sekundarstufe-IIAbschluss (unter „A-Level“, d.h. ohne abgeschlossene Sekundarschulbildung) und doppelt so groß wie bei Abiturienten. 


\section{Über die Wechsel- wirkungen zwischen Bildung und subjek- tivem Wohlbefinden und Glück wird immer mehr geschrieben.}

Die verfügbaren Informationen legen den Schluss nahe, dass die Bildung sowohl unmittelbar als auch auf lange Sicht positive Auswirkungen darauf hat, inwieweit sich der Einzelne selbst als glücklich einschätzt. In Blanchflower und Oswald (2000) ist von Schätzungen der „Glücksgleichungen“ in Regressionsanalysen die Rede, in denen der berichtete Glückszustand einer Person zu verschiedenen persönlichen Merkmalen in Bezug gesetzt wird. Sie kommen zu dem Ergebnis, dass das Bildungsniveau mit einem größeren Glücksgefühl verbunden wird, selbst wenn in der Untersuchung die Variable Familieneinkommen unberücksichtigt bleibt. Möglich ist auch, dass das Bildungsniveau einer Person positive Auswirkungen auf das Glücksgefühl anderer hat, in diesem Fall investieren egoistische Personen vom gesellschaftlichen Standpunkt aus betrachtet in der Regel möglicherweise zu wenig in die Bildung. Umgekehrt kann die Bildung aber auch das Glücksgefühl beeinflussen, da sie den Blick jedes Einzelnen auf seine Stellung im Vergleich zu anderen verändert; in diesem Fall könnten die Ergebnisse von Blanchflower und Oswald die Effekte einer Ausweitung des Bildungsangebots auf das Wohlbefinden überzeichnen. Indessen stellt Putnam (2001) unter Verwendung von Daten aus verschiedenen US-Bundesstaaten fest, dass sowohl das persönliche Bildungsniveau als auch das durchschnittliche Bildungsniveau auf County-Ebene positive Auswirkungen auf den Glückszustand haben.

\subsection{Schlussbetrachtungen}

Mehr Bildung zahlt sich deutlich aus ...

Bildung, Fortbildung und Lernen kommt bei der Schaffung der Grundvoraussetzungen für Wirtschaftswachstum, sozialen Zusammenhalt und persönliche Entwicklung eine ganz entscheidende Rolle zu. Humankapitalinvestitionen entfalten ihre Wirkung progressiv und zahlen sich erst nach einer gewissen Zeit aus. Sofern die Auswirkungen messbar und vergleichbar sind, legen einige Untersuchungen den Schluss nahe, dass die gesellschaftlichen Effekte des Lernens (auf Gesundheit, Kriminalität, sozialen Zusammenhalt) ebenso groß sein könnten wie die Auswirkungen auf die Wirtschaftsproduktivität, wenn nicht sogar größer. Dennoch liefert die Korrelation zwischen den Gesamtindikatoren für die Schulbildung und verschiedenen wirtschaftlichen und sozialen Ergebnissen keinen Hinweis darauf, in welche Richtung sich das öffentliche Bildungswesen entwickeln soll, abgesehen von der Beobachtung, dass mehr Lernen generell von Nutzen ist. Zwischen dem Lernen und anderen Dimensionen des sozialen, institutionellen und rechtlichen Umfelds bestehen Synergieeffekte und Komplementaritäten. Qualifikationen und Kompetenzen können einen indirekten Einfluss ausüben, indem sie den Effekt anderer Faktoren verstärken. Die Hochschulbildung kann in Partnerschaft mit öffentlichen und privaten Interessen eine entscheidende Rolle bei der Förderung von Forschung und Innovation spielen, die ihrerseits ein rascheres Wachstum des Volkseinkommens begünstigen.

Im vorliegenden Kapitel wurde eine potentiell starke Interaktion zwischen Human- und Sozialkapital ermittelt. Das Lernen und die Vorbereitung auf den Lernprozess, die innerhalb der Familien und lokalen Gemeinschaften beginnen, stellen eine wichtige Grundlage für die kontinuierliche Weiterentwicklung des Humankapitals auf dem Wege der regulären Bildung wie auch des lebensbegleitenden Lernens dar. Soziale Netzwerke und Lerneinrichtungen können 


\author{
... und die Qualität und \\ Verteilung von \\ Lernmöglichkeiten sind \\ von Bedeutung.
}

Bei den Messungen des Humankapitals muss den effektiven Qualifikationen und Inputfaktoren, wie auch der in Bildungsmaßnahmen verbrachten Zeit Rechnung getragen werden. auch das informelle Lernen „am Arbeitsplatz“ und im Alltag fördern. Die Wechselwirkungen zwischen Human- und Sozialkapital stellen sich aber nicht automatisch ein.

Eine Steigerung des Humankapitals insgesamt muss außerdem von Strategien flankiert werden, die an folgenden Punkten ansetzen:

1. Qualität der Humankapitalinvestitionen und Anpassung der Qualifikationen an den sozialen und wirtschaftlichen Bedarf;

2. Verteilung der Lernmöglichkeiten innerhalb der Länder angesichts der zwischen Ungleichheit und Gesamtergebnissen bestehenden Zusammenhänge sowie

3. Gefahr von „Unterinvestitionen“ des Markts angesichts bestimmter Eigenschaften des Humankapitals, die diesem Züge eines „öffentlichen Guts“ bzw. von „Externalitäten“ verleihen.

Angesichts des wachsenden Politikkonsenses über die Bedeutung des lebenslangen und lebensumfassenden Lernens (letzteres bezieht sich auf ein breites Spektrum an Lernumgebungen) wird man sich schließlich der Probleme bewusst, die sich aus einer zu starken Konzentration auf enge Interpretationen und Messgrößen des Humankapitals ergeben. Ergebnisorientierte Messungen, wie beispielsweise Erhebungen über die Lese- und Schreibkompetenzen sowie die Rechenfertigkeit, sind selbstverständlich ein sehr wichtiges Instrument zur direkten Messung des Humankapitals. Angesichts der komplementären Vor- und Nachteile traditioneller inputorientierter Messungen des Humankapitals und direkter, auf Erhebungen fußender Messungen muss jede globale Messstrategie beiden Konzepten gebührend Rechnung tragen. 


\section{Anmerkungen}

1. Inglehart (1997) definiert Kultur als ein „System von Einstellungen, Wertmaßstäben und Kenntnissen, die von vielen innerhalb einer Gesellschaft geteilt und von Generation zu Generation übertragen werden“ (S. 15). Kultur wird erworben und verändert sich nur recht langsam. Laut Inglehart wandelt sich die Kultur stärker durch einen Generationenwechsel als eine Neuorientierung der Werte und Einstellungen der Menschen, die seit ihrer frühen Kindheit bestimmte Auffassungen vertreten und Wertvorstellungen haben.

2. Humankapital und Sachkapital haben in dem Maße mehrere Merkmale gemeinsam, wie das Humankapital $a$ ) eine zeitliche Dimension enthält, in der Investitionen getätigt werden und Nutzeffekte entstehen, und $b$ ) auf die Erzielung kurzfristiger Vorteile verzichtet wird. Es unterscheidet sich aber vom Sachkapital in dem Maße, wie die allgemein gültigen Regeln der Veräußerlichkeit und Verbrauchersouveränität, durch die Verbraucher ein externes Gut erwerben und „besitzen“ können, keine Anwendung finden. Per definitionem ist das Humankapital dem Menschen eigen, und die von ihm produzierten Dienstleistungen können am Markt verkauft werden; streng genommen kann das Eigentum an Humankapital aber nicht übertragen oder veräußert werden, außer im Fall menschlicher Sklaverei.

3. Berechnen lässt sich der Wert des Humankapitalbestands, indem vom Gegenwartswert künftiger Nettogehaltserhöhungen, die der Teilnahme an zusätzlichen Bildungs- und Fortbildungsmaßnahmen zu verdanken sind, die Investitionskosten in diese zusätzliche Bildung und Fortbildung während eines gesamten Lebenszyklus subtrahiert werden und das Ergebnis um eine soziale Rendite aktualisiert wird, die den Opportunitätskosten entspricht, die durch Investitionen in weiterführende Bildungsmaßnahmen entstehen.

4. Auch wenn das Humankapital als Eigentum oder Merkmal des Menschen definiert wird, führt die Summe bzw. Kombination der verschiedenen Quantitäten an individuellem Humankapital zu einer institutionellen Kompetenz, deren Einfluss stärker und weitreichender ist als der ihrer Einzelkomponenten.

5. Übergreifeffekte entstehen, wenn die Verbesserung der Qualifikationen einiger nicht nur eine Produktivitätssteigerung dieser qualifizierten Personen, sondern auch jener nach sich zieht, denen der Produktivitätszuwachs der Höherqualifizierten zugute kommt.

6. Mit dem „Policy-mechanism“-Konzept sollen jene spezifischen Inputs einzelner Schulen identifiziert werden, die für große Ergebnisunterschiede zwischen den Schulen und dem jeweiligen Lernerfolg sorgen.

7. Gemäß dem „Classroom-culturist“-Konzept spielen die in einer Klasse maßgeblichen Normen und die dort stattfindende Sozialisierung für das Verständnis der Ergebnisse und die Effektivität der Schule eine wichtige Rolle.

8. Gundlach, Wossmann und Gmelin (2000) gehen auf der Basis internationaler Erhebungsdaten über Schülerleistungen in Mathematik und Naturwissenschaften aus dem Zeitraum 1970-1994 davon aus, dass die Produktivität der Schule im besten Fall trotz der Ausgabensteigerung je Schüler in neun von elf untersuchten OECD-Ländern nicht zugenommen hat.

9. In jüngster Zeit sind einige Versuche unternommen worden, um Ersatzvariable für das Kulturkapital zu ermitteln. Bisher haben sich diese auf externe Familienaktivitäten konzentriert (z.B. Besuch eines Symphoniekonzerts, Besichtigung einer Kunstgalerie, Reisen), sich allwöchentlich zuhause wiederholende Aktivitäten (z.B. Diskussionen in der Familie, Abendessen, Debatten über Geschehnisse außerhalb des häuslichen Bereichs), die Art von Lesestoff, der zuhause verfügbar ist (z.B. Tageszeitung, Enzyklopädie, Gedichtbände) wie auch die Sprachen, die von Mutter und Vater verstanden und gesprochen werden (vgl. Willms, 1999).

10. Beispiele für innovative Praktiken bei der Ansiedlung von Lernmöglichkeiten in unmittelbarer Nähe der Orte, an denen Menschen arbeiten, leben und an denen sie sich bei Reisen aufhalten, werden in der Veröffentlichung ,How Adults Learn" (OECD und US-Bildungsministerium, 1998) vorgestellt.

11. Bei den Ländern handelte es sich um: Deutschland, Korea, Japan, Schweden, das Vereinigte Königreich und die Vereinigten Staaten.

12. Field und Spence (2000) kommen in ihrer Studie über die Erwachsenenbildung in Nordirland zu der Schlussfolgerung, dass enge soziale Bindungen in Familien und Religionsgemeinschaften zwar mit guten Leistungen in der Erstausbildung verbunden sind, diese Bindungen gleichzeitig aber der Teilnahme an formalen Weiterbildungsaktivitäten für Erwachsene im Wege stehen können. Dies ist möglicherweise z.T. dadurch bedingt, dass die Teilnahme an Maßnahmen der regulären Fort- oder Weiterbildung die engen familiären Bindungen und Kontakte in der Gemeinschaft ersetzt. Fehlt das Sozialkapital, das zwischen den verschiedenen Gemeinschaften Brücken schlägt, werden Individuen und lokalen Gruppen möglicherweise kaum Anreize geboten, an formaleren und stärker strukturierten Bildungs- und Fortbildungsmaßnahmen teilzunehmen. Gleichwohl können solide Sozialkapitalbeziehungen innerhalb der Gemeinschaft (entsprechend dem 
in Kapitel 3 weiter unten erörterten Konzept der „Verwandtschaftsbeziehungen“) einem höheren Maß an informeller Erwachsenenbildung Vorschub leisten, da sich die Erwachsenen bei ihren Informationsaktivitäten und ihrem sozialen Engagement auf das Familien- und Gemeinschaftsnetz stützen.

13. Bundesrepublik Deutschland, England und Wales, Israel, Italien, Japan, Niederlande, Polen, Schweden, Schweiz, Taiwan, Tschechoslowakei, Ungarn und Vereinigte Staaten.

14. Der IALS wurde mit Personen zwischen 16 und 65 Jahren durchgeführt, um die Fähigkeit Erwachsener zu testen, gedruckte und geschriebene Informationen zu nutzen. Die Lesekompetenz bezieht sich auf die Fähigkeit, kontinuierliche Texte zu lesen und zu verstehen; das Verständnis von Dokumenten stellt auf die Fähigkeit ab, Berichte, Dokumente und verschiedene Formen nicht kontinuierlicher Texte zu interpretieren, während die quantitative Lesekompetenz darin besteht, quantitative Informationen wie mathematische Abbildungen und Grafiken zu interpretieren, die gewöhnlich in einen kontinuierlichen Text eingebettet sind. Gemessen wurden die Lese- und Schreibkompetenzen nicht in einer dichotomen Unterscheidung zwischen Lese- und Schreibkundigen und Analphabeten, sondern anhand eines Gesamtkatalogs messbarer Kompetenzen.

15. Weiss (1995) wie auch viele andere betrachten die theoretische Verallgemeinerung und empirische Validität von „Signalling"-Modellen unter einem ganz anderen Blickwinkel.

16. Bynner et al. (2001) beobachteten ferner, dass eine Verbesserung der Grundqualifikationen zur Erreichung der nationalen Kompetenzziele Erwachsener im Jahr 2010 dem Steuerzahler durch die Verbesserung der Rechenfertigkeiten sowie Lesekompetenzen Ersparnisse in Höhe von 2,5 Mrd. £ bzw. 0,4 Mrd. £ ermöglichen könnte.

17. Temple (2001) stellt fest: „Nehmen wir beispielsweise die Behauptung, ein x-prozentiges Wachstum in einem gegebenen Land sei einer Steigerung der Qualität der Erwerbsbevölkerung zu verdanken. Das bedeutet nicht, dass die Zuwachsrate der gesamtwirtschaftlichen Produktion ohne diese qualitative Verbesserung der Erwerbsbevölkerung unbedingt um eben genau diese x-Prozentpunkte niedriger gelegen hätte. In Wirklichkeit kann das Bildungsniveau über die Erwerbsbeteiligung, Investitionen, ja sogar Forschung und Entwicklung sowie des Wachstums der gesamten Faktorproduktivität auch andere, indirekte Auswirkungen auf die gesamtwirtschaftliche Produktion haben. Empirische Wachstumsratenanalysen erfassen diese indirekten Effekte nicht, d.h. die für das Wachstum insgesamt wichtige Bedeutung bestimmter Variablen wie Bildung wird unweigerlich übergangen“.

18. Beispielsweise hängt das Verhältnis zwischen Studienjahren und Produktion davon ab, ob Niveau, Veränderungsquotienten oder einfache logarithmische Darstellungen gewählt werden. Es kann nicht davon ausgegangen werden, dass die Bildungsrenditen vom Ausgangsniveau unabhängig sind.

19. Pritchett verwendet auch die Zuwachsrate der gesamten Faktorproduktivität in empirischen Wachstumsratenanalysen und kommt im Wesentlichen zur gleichen Schlussfolgerung.

20. Barro und Sala-i-Martin (1995) wie auch Barro und Lee (1997) stellen fest, dass zwischen dem ursprünglichen Niveau der Frauenbildung (sowohl Sekundar- als auch Hochschulbildung) und dem Wachstum offenbar ein negativer Zusammenhang besteht.

21. Andere Studien deuten auf eine starke Korrelation zwischen dem Bildungsniveau der Mutter und dem Gesundheitszustand ihrer Kinder, bezogen auf deren gesamte Lebenszeit, hin.

22. Die bekannte Barro-Lee-Datenreihe liefert chronologische Daten über die Bildungsabschlüsse in der Bevölkerung der über 25-Jährigen. Erstellt wurde dieser Datensatz weitgehend auf der Grundlage von Zensusergebnissen, die von der Statistikabteilung der Vereinten Nationen gesammelt wurden, wobei die Angaben für fehlende Jahre durch Schätzungen ergänzt wurden.

23. Bei einem größeren Stichprobenumfang von Ländern kamen sie zu dem Ergebnis, dass die Hochschulbildung bedeutende Auswirkungen hat - so zieht eine Verlängerung der Sekundarschulzeit junger Männer um durchschnittlich 0,68 Jahre einen Anstieg des Jahreswachstums um 1,1 Prozentpunkte jährlich nach sich, während eine Verlängerung der Hochschulbildung um 0,09 Jahre im Durchschnitt für eine Erhöhung des Jahreswachstums um 0,5 Prozentpunkte sorgt.

24. Unter Verwendung von Daten für 78 Länder untersucht McMahon die Interaktion zwischen verschiedenen sozialen Zielen wie Gesundheit, Demokratisierung, Abbau der Ungleichheiten in der Einkommensverteilung und Armut, Umwelteffekte sowie Kriminalität. Er kommt zu dem Ergebnis, dass über die Hälfte der gesamten Bildungseffekte durch induzierte indirekte Effekte bedingt ist, wie eine Verbesserung der Gesundheit, die sich ihrerseits wieder positiv auf das Wachstum der Pro-Kopf-Einkommen auswirkt.

25. Es ist also möglich, die Informationen über offenkundige Präferenzen einzelner Personen für den Erwerb eines bestimmten Gesundheitsniveaus mit privaten Mitteln zu verwenden, um den monetären Gegenwert einer Anhebung des Bildungsniveaus zu berechnen, indem der Geldwert von Verbesserungen verschiedener sozialer Faktoren addiert wird, darunter ein Rückgang der Kriminalität, eine Verbesserung des Gesundheitszustands etc.

26. Bynner et al. (2001), Bynner und Egerton (2001) sowie Schuller et al. (2001).

27. Dieses Ergebnis erklärt sich aus der Tatsache, dass höher gebildete Arbeitskräfte im Durchschnitt mit einer größeren Kontinuität in einem Beschäftigungsverhältnis bleiben und dass die steuerliche Grenzbelastung dieser Arbeitskräfte auf Grund des höheren Gehalts proportional größer ist. 

Kapitel 3

\section{DIE ROLLE DES SOZIALKAPITALS}

„Praktisch allen kommerziellen Transaktionen inhärent ist ein Element des Vertrauens, das gilt auf jeden Fall für solche Transaktionen, die sich über einen gewissen Zeitraum erstrecken. Es lassen sich plausible Argumente für die These anführen, dass die wirtschaftliche Rückständigkeit in der Welt zu einem großen Teil auf einen Mangel an gegenseitigem Vertrauen zurückzuführen ist“. Kenneth J. Arrow, „Gifts and Exchanges“, Philosophy and Public Affairs, 1, Sommer 1972, S. 357.

\subsection{Einleitung}

Neben den

Kompetenzen des Einzelnen spielen auch die sozialen Beziehungen eine wichtige Rolle für das, was ein Mensch zu leisten imstande ist.
Das Sozialkapital besteht aus sozialen Beziehungen und kann in seiner Eigenschaft als Kapital als Ressource betrachtet werden, in die wir investieren, um uns eine Fülle von Vorteilen zu verschaffen.
Gesellschaften, die auf einem Netz von Vertrauen und Zusammenarbeit aufbauen, können zur Realisierung des Humanpotentials beitragen. In der ökonomischen Fachliteratur wird man sich immer mehr der Bedeutung bewusst, die sozialen Netzen und der Existenz eines Vertrauensklimas für die Unterstützung kollektiver Bemühungen zukommt. Temple (2001) merkte beispielsweise zum Sozialkapital an, dass es ,ein nützlicher Oberbegriff für jene gesellschaftlichen Aspekte ist, die, so schwer sie sich auch messen und in formale Modelle integrieren lassen, doch weithin als wichtige Bestimmungsfaktoren für langfristige wirtschaftliche Erfolge anerkannt werden. Für einige (wenn auch nicht alle) Ökonomen ist die intuitive Einsicht, dass es , auf die Gesellschaft ankommt' so zwingend, dass dies schwerer für sie wiegt, als die Tatsache, dass es bisher kaum irgendwelche theoretischen Grundlagen zur Erhärtung dieser These gibt“".

Im vorliegenden Kapitel werden Definition, Messgrößen und Effekte des Sozialkapitals untersucht. Da dieser Begriff verhältnismäßig neu ist und noch kein allgemeiner Konsens über seine Bedeutung besteht, wird in diesem Kapitel ein möglicher Ansatz zur Definition des Konzepts und zur Messung seines Beitrags zum menschlichen Wohlergehen skizziert. Auch wenn die bisher verfügbaren Informationen nur vorläufigen Charakter haben, deuten sie doch darauf hin, dass das Sozialkapital ein für die Gestaltung der öffentlichen Politik nützliches Konzept ist und dass es weiterer Arbeiten zur Begriffsentwicklung und zur Konzipierung von Messtechniken bedarf.

\subsection{Verschiedene Konzepte des Sozialkapitals}

Das Konzept des „Sozialkapitals“ unterscheidet sich von dem des Humanund Sachkapitals in mehrerlei Hinsicht:

- Es besteht aus Beziehungen, ist also nicht das exklusive Eigentum von Individuen.

- Es stellt im Wesentlichen ein öffentliches Gut dar, weil es einer Gruppe von Menschen gemeinsam gehört. 
Das Sozialkapital hängt von den Aktionen der Individuen ab, die sich dessen Förderung zum Ziel setzen.

\section{Die Wurzeln dieses Konzepts liegen in frïheren Forschungs- arbeiten.}

- Es ist das Produkt gesellschaftlicher Investitionen von Zeit und Energie, wenn auch auf weniger unmittelbare Weise, als dies beim Humanoder Sachkapital der Fall ist.

Das Sozialkapital ist auch das Produkt von ererbten kulturellen Werten sowie von Verhaltensnormen. Es besitzt also eine soziale Dimension, insofern es nicht in Individuen, sondern in Beziehungen wurzelt, und eine Dimension des Kapitals, insofern es eine Ressource darstellt, die der Gesellschaft auf längere Sicht eine Fülle von Vorteilen bringen kann. Es kann aber auch die Ursache von Funktionsstörungen sein, wenn es von einer Gruppe als Waffe gegen andere Gruppen eingesetzt wird.

Viele Autoren, die den Begriff „Sozialkapital“ verwenden, haben zum besseren Verständnis der Anreize, die Menschen zur Nutzung von Sozialkapital und zu Investitionen in dessen Erwerb bewegen, einen individualistischen Ansatz gewählt (Glaeser, 2001). Coleman sah im Sozialkapital bis zu einem gewissen Grad ein Instrument zur Erreichung bestimmter Ziele. Andere, darunter auch Hirschman, hoben vor allem die Bemühungen von Individuen um die gemeinsame Verwirklichung nicht geldwerter Ziele hervor, wie Gerechtigkeit, Schönheit, Liebe, Gemeinschaft und Freundschaft. Dieser nicht instrumentale Aspekt kollektiven Handelns stelle ,eine Investition in die Identität von Individuen und Gruppen“ dar (Hirschman, 1984). Hirschman befasst sich insbesondere mit dem Wert der "Sozialenergie“ in Form von Freundschaft, gemeinsamen Idealen und Vorstellungen, die über das rationale Eigeninteresse und kommerzielle Transaktionen hinausgehen und die soziale Zusammenarbeit fördern. Unabhängig von den Motiven, die für Zusammenarbeit und Vertrauen maßgeblich sind, können Investitionen in die Identität von Individuen und Gruppen zur Schaffung dichter sozialer Netzwerke und letztlich zu besseren wirtschaftlichen und sozialen Ergebnissen führen. Auf diese Weise können sich bürgergesellschaftliches Engagement, Ehrlichkeit und Sozialvertrauen gegenseitig verstärken.

Die Idee des Sozialkapitals kann bis zu den Arbeiten von Alexis de Tocqueville $^{1}$, Emile Durkheim ${ }^{2}$ und Max Weber $^{3}$ zurückverfolgt werden (wegen eines umfassenden Überblicks über die historischen Wurzeln des Konzepts vgl. Woolcock, 1998). Die erste bekannte Verwendung des Begriffs „Sozialkapital“ in seiner gegenwärtigen Bedeutung erfolgte im Zusammenhang mit der Rolle des Sozialkapitals für Bildung und lokale Gemeinschaften (Hanifan, 1916). Seitdem wurde der Begriff des Sozialkapitals von Jacobs $(1961)^{4}$ in ihrer Analyse der Stadtviertel, von Loury (1987) in einer Arbeitsmarktstudie, von Coleman (1988), der die Komplementarität zwischen Sozialund Humankapital herausstellte, sowie von Putman (1993) und Fukuyama (1995) verwendet, die das Konzept auf nationalstaatlicher oder regionaler Ebene anwendeten (wobei Putman vor allem die Rolle des bürgerlichen Engagements für die Förderung der Demokratie und des sozialen Zusammenhalts hervorhob). Bourdieu (1979) sowie Bourdieu und Passeron (1970) verwendeten ihrerseits das hiermit eng verbundene Konzept des „Kulturkapitals“ (vgl. Abschnitt 2.4, Kapitel 2).

Es gibt keine allgemein gültige Definition von Sozialkapital. Das Konzept lässt sich aus mindestens vier großen Blickwinkeln betrachten:

1. Aus der anthropologischen Literatur stammt die Idee, dass der Mensch einen natürlichen Assoziationsinstinkt besitzt. Fukuyama (1999) hebt

(C) OECD 2004 
beispielsweise hervor, dass die Sozialordnung ein biologisches Fundament hat und die Wurzeln des Sozialkapitals in der menschlichen Natur liegen.

2. In der soziologischen Literatur werden die gesellschaftlichen Normen und die Quellen der menschlichen Motivation beschrieben. Der Schwerpunkt liegt dabei auf Merkmalen der sozialen Organisation, wie Vertrauen, Normen der Gegenseitigkeit und Netzen des bürgerlichen Engagements.

3. Die ökonomische Fachliteratur geht von der Annahme aus, dass Menschen ihren persönlichen Nutzen zu optimieren suchen, indem sie beschließen, mit anderen in Interaktion zu treten und zur Durchführung verschiedener Arten von Gruppenaktivitäten auf Sozialkapitalressourcen zurückzugreifen (Glaeser, 2001). Bei diesem Ansatz liegt der Schwerpunkt auf den Investitionsstrategien von Individuen, die in Bezug auf die Nutzung ihrer Zeit zwischen verschiedenen Alternativen wählen können.

4. Eine Strömung in der politikwissenschaftlichen Literatur hebt die Rolle der Institutionen sowie der politischen und sozialen Normen als Bestimmungsfaktoren für das menschliche Verhalten hervor. In jüngsten Arbeiten der Weltbank über die Rolle des Sozialkapitals bei der Armutsbekämpfung und die Förderung einer nachhaltigen Entwicklung wurde die Bedeutung von Institutionen, sozialen Vereinbarungen, Vertrauen und Netzwerken unterstrichen ${ }^{5}$.

Das Konzept des Sozialkapitals wurde auch mit globaleren makroinstitutionellen Aspekten in Verbindung gebracht, die von einigen Ökonomen als „soziale Fertigkeiten“ bezeichnet werden (z.B. Abramovitz und David, 1996; Omori, 2001; Hall und Jones, 1999; Temple und Johnson, 1998). Putnam (2001a), Woolcock (2001) und Knack (1999) bevorzugen indessen eine enger gefasste Definition, die sich auf die sozialen Netzwerke und die damit direkt verbundenen Normen der Gegenseitigkeit (oder des Vertrauens im Fall von Knack) konzentriert. Einige Kommentatoren (z.B. Woolcock, 2001) betrachten Vertrauen als Ertrag des Sozialkapitals (d.h. der sozialen Netze und der damit verbundenen Normen), während für andere Autoren das Vertrauen eine Komponente der gemeinsamen Wertvorstellungen und Normen darstellt, die das Sozialkapital bilden.

Der vorliegende Bericht

Im vorliegenden Bericht wird das Sozialkapital definiert als Netzwerkbietet eine eigene Definition von Sozialkapital an. beziehungen zusammen mit gemeinsamen Normen, Wertmaßstäben und Überzeugungen, die die Zusammenarbeit in oder zwischen den Gruppen erleichtern. Netzwerke hängen mit dem objektiven Verhalten von Akteuren zusammen, die in Verbänden und Vereinigungen aktiv sind. Gemeinsame Normen, Wertmaßstäbe und Überzeugungen beziehen sich auf die subjektiven Einstellungen und Verhaltensweisen von Individuen und Gruppen, ebenso wie auf Sanktionen und Verhaltensegeln, die auf breiter Basis geteilt werden ${ }^{6}$. Der kulturelle Kontext, in dem die gemeinsamen Einstellungen, Wertmaßstäbe und Kenntnisse von Generation zu Generation übertragen werden, trägt wesentlich zum Verständnis der Entscheidungen bei, die Individuen und Gruppen in Bezug auf die Form der Zusammenarbeit treffen. Gemeinsame Normen und Wertmaßstäbe ermöglichen es Menschen, miteinander zu kommunizieren und gemeinsame Erfahrungen wie auch Differenzen in Bezug auf gewisse Normen und Wertvorstellungen zu 


\section{Vertrauen begünstigt die Entwicklung von Sozialkapital.}

Zuneigungs-, Brücken- und Kontaktbeziehungen sind wichtige Dimensionen des Sozialkapitals. interpretieren. Unterschiedliche Wert- und Bedeutungssysteme können nebeneinander existieren, ohne dass dadurch die Zusammenarbeit unbedingt gefährdet zu werden braucht, sofern ein Klima der Toleranz besteht. Daher sind ein auf Toleranz gegenüber anderen Kulturen und Überzeugungen fußender Dialog wie auch das gegenseitige Verständnis wichtige Dimensionen des sozialen Zusammenhalts und tragen zur Stärkung des Sozialkapitals bei.

Vertrauen kann zugleich als Quelle und Ertrag des Sozialkapitals betrachtet werden, stellt darüber hinaus aber auch eine sehr gute Ersatzvariable für viele Normen, Überzeugungen und Werte dar, auf die sich die soziale Zusammenarbeit stützt. Es kann unterschieden werden zwischen Menschen, die anderen vertrauen, und Menschen, die vertrauenswürdig sind. Vertrauen ist möglicherweise ein guter Indikator für Vertrauenswürdigkeit (unter Berücksichtigung von Zeitverzögerungen), doch beschreibt Vertrauenswürdigkeit ein Verhalten, das sich aus einer Vielzahl von Faktoren, darunter auch den Netzwerken und gemeinsamen Wertmaßstäben und Normen, ergibt. Es gibt drei Formen des Vertrauens:

- das Vertrauen zwischen Personen, die sich gut kennen (Familie, enge Arbeitskollegen und Nachbarn);

- das interpersonelle Vertrauen zwischen „Fremden“ sowie

- das Vertrauen in öffentliche und private Institutionen.

Das Sozialkapital ermöglicht es Individuen, Gruppen und Gemeinschaften, gemeinsame Probleme leichter zu lösen. Die Reziprozitätsnormen und die Netzwerke sorgen für ein kollektiv wünschenswertes Verhalten. Wenn diese beiden Faktoren nicht gegeben sind, neigt der Einzelne in der Regel nicht zur Kooperation, da er sich nicht darauf verlassen kann, dass die anderen ebenfalls kooperieren. Das Sozialkapital kann ein Nebenprodukt verschiedener sozialer Aktivitäten sein, die nicht unbedingt auf die Stärkung des Sozialkapitals ausgerichtet $\operatorname{sind}^{7}$. Ebenso wie das Humankapital hat auch das Sozialkapital „positive Externalitäten“, dergestalt, dass der Beitrag eines Einzelnen oder einer Gruppe zum Sozialkapital vielen Menschen zugute kommt. Mithin besteht die Gefahr der Unterinvestition, da die Akteure, die zum Sozialkapital beitragen, sich dessen Nutzeffekte nicht voll zu Eigen machen. Wie Coleman zu bedenken gab: „Im Ergebnis wird es in der Gesellschaft ein Ungleichgewicht geben zwischen den relativen Investitionen in Organisationen, die Individualgüter für den Markt produzieren, und Organisationen (häufig freiwilligen Verbänden), die Vorteile ,produzieren', die nicht voll ausgeschöpft werden" (Coleman, 1990, S. 317). Jedoch verschaffen der Zugang zu Informationen und zur Einflussnahme über die sozialen Netzwerke dem Einzelnen private Vorteile, und dieser Zugang kann in einigen Fällen von Individuen oder Gruppen zur Ausgrenzung anderer Akteure bzw. zur Stärkung der eigenen Dominanz bzw. Privilegien genutzt werden.

Woolcock (1999) hat drei wichtige Dimensionen des Sozialkapitals identifiziert. Zuneigungsbeziehungen zwischen Mitgliedern einer Familie bzw. einer ethnischen Gruppe, Brückenbeziehungen zwischen entfernten Freunden, Geschäftspartnern und Kollegen sowie Kontaktbeziehungen zwischen verschiedenen sozialen Schichten, zu denen unterschiedliche Gruppen in der Hierarchie von Macht, sozialem Status und Wohlstand Zugang haben. Woolcock (2001) versteht unter der letztgenannten Art von Beziehungen die Fähig- 
Sowohl die Zuneigungsals auch die Brïckenbeziehungen sind notwendig, um ein Auseinanderbrechen des sozialen Gefüges zu verhindern.

Gruppen mit starkem Zusammenhalt können Außenstehende ausschließen.

Über die Nachteile gewisser Formen des Sozialkapitals ist man sich durchaus im Klaren. keit von Individuen und Gruppen, Ressourcen, Ideen und Informationen zu nutzen, die von formalen Institutionen außerhalb ihres unmittelbaren Umfelds ausgehen.

Wenn enge Zuneigungsbeziehungen bestimmten Gemeinschaften oder Gruppen auch ein Identiäts- und Gemeinschaftsgefühl verleihen, können sie doch ohne Brückenbeziehungen, die über verschiedene soziale Grenzen (Religion, ethnische Zugehörigkeit, sozioökonomischer Status) hinausreichen, dazu führen, dass sich der Gesichtskreis der betreffenden Personen verengt und Außenseiter bewusst ausgegrenzt werden. Relativ homogene Gruppen zeichnen sich durch ein hohes Maß an Vertrauen und Zusammenarbeit innerhalb der Gruppe, aber nur geringes Vertrauen und geringe Bereitschaft zur Zusammenarbeit mit den übrigen Mitgliedern der Gesellschaft aus. Gewisse Formen exklusiver, auf Zuneigung beruhender Beziehungen können dem sozialen Zusammenhalt und der persönlichen Entfaltung im Wege stehen. In diesen Fällen sind die Brückenbeziehungen schwach, die Zuneigungsbeziehungen indessen stark. Powell und Smith-Doerr (1994) stellten in diesem Zusammenhang fest, dass „Beziehungen, die Personen aneinander binden, auch zu Beziehungen werden können, die den Blick für andere Personen verstellen“.

Ist der Vertrauensradius in einer „verschworenen“ Gemeinschaft, wie im Falle einer Familie oder eines engen Freundeskreises, begrenzt, so besteht die Gefahr, dass soziale Interaktionen gefördert werden, die nach innen gekehrt und weniger auf Vertrauen und Zusammenarbeit mit den übrigen Mitgliedern der Gemeinschaft ausgerichtet sind (Knack, 1999; Portes und Landholt, 1996). Eine ausschließliche Fokussierung auf Gruppeninteressen zu Lasten allgemeinerer öffentlicher Interessen kann sozial zerstörerische ,Rent-seeking“-Aktivitäten fördern (Olson, 1982, und Knack, 1999). In Unternehmen können starke Beziehungen, die auf Vertrauen und gegenseitiger Verpflichtung beruhen, unter gewissen Umständen von außen kommende Informationen abblocken und Innovationen im Wege stehen (Kern, 1998, und Uzzi, 1997).

Auch wenn ethnische Beziehungen eine „Quelle anpassungsfördernder Vorteile" sein können, wenn Zuwanderergruppen in einem neuen Land ankommen, können doch andererseits exklusive ethnische Beziehungen den Einzelnen daran hindern, seine Kontakte in einem größeren Netzwerk weiter auszubauen. Das unterstreicht die Bedeutung von Brückenbeziehungen in jenen Fällen, in denen eine Vielfalt ethnischer und sonstiger Gruppen existiert.

Bestimmte Formen von Zuneigungsbeziehungen können den sozialen $\mathrm{Zu}$ sammenhalt unter gewissen Umständen erschweren. In dieser Hinsicht unterscheidet sich das Sozialkapital nicht von anderen Kapitalformen, die jeweils zu unterschiedlichen Zwecken eingesetzt werden können - von denen nicht alle unbedingt im Interesse der Gesellschaft insgesamt liegen. Einige in besonderem Maße „verschworene“ Gruppen, wie beispielsweise Drogenkartelle, Schlepperbanden, Mafiaorganisationen oder terroristische Gruppen, zeichnen sich manchmal durch ein intern hohes $\mathrm{Ma} \beta$ an Vertrauen und Gegenseitigkeit aus . Dieselben Gruppen können aber auch Individuen umfassen, die über ein hohes Humankapitalniveau verfügen und die Finanz- und sonstige Kapitalformen für gesellschaftlich zerstörerische und unerwünschte Zwecke einsetzen. Einige Arten zu exklusiver, sozialer Beziehungen auf nationaler oder regionaler Ebene untergraben möglicherweise das gesellschaftliche Gefüge? 
Die Messung des Sozialkapitals steckt noch immer in den Kinderschuhen ...
... es werden derzeit aber ganz, neue Messinstrumente entwickelt. stellen aber nicht grundsätzlich das Potential des Human- bzw. Sozialkapitals in Frage, in anderen Fällen Nutzeffekte für alle oder doch die meisten Mitglieder der Gesellschaft hervorzubringen. Die Vorteile, die bei fast allen Formen enger sozialer Beziehungen gegeben sind, überwiegen bei weitem die negativen Effekte.

\subsection{Wie messen wir Sozialkapital?}

Sozialkapital lässt sich schwer messen. In der Regel beziehen sich die meisten der verfügbaren Messungen des Sozialkapitals auf Werte wie Vertrauen und Engagement bzw. Interaktionen in gesellschaftlichen bzw. Gruppenaktivitäten. Putman (2000a) hat in seiner Analyse der Disparitäten des Sozialkapitals zwischen den US-Bundesstaaten auf ein breites Spektrum von Querschnitts- und Zeitreihendaten zurückgegriffen. Seine Messungen des Sozialkapitals fußen generell auf einem zusammengesetzten Index, der sich aus folgenden Komponenten zusammensetzt: $a$ ) Intensität der Teilnahme am Gemeinschafts- und Organisationsleben, $b$ ) öffentliches Engagement (z.B. Teilnahme an Wahlen), c) ehrenamtliche Tätigkeiten, $d$ ) informelle Geselligkeit (Treffen mit Freunden) sowie $e$ ) subjektives Niveau des zwischenmenschlichen Vertrauens.

Prinzipiell sollten die Messgrößen des Sozialkapitals a) so viele Kerndimensionen wie nur eben möglich abdecken (Netzwerke, Wertmaßstäbe und Normen) und $b$ ) Ausgewogenheit zwischen subjektiven, von der persönlichen Einstellung abhängigen Elementen einerseits (z.B. individuell bekundetes Vertrauensniveau) und Verhaltensaspekten andererseits (z.B. Mitgliedschaft in Verbänden und Ausmaß der sozialen Beziehungen) sicherstellen. Bei der Anwendung dieser Messgrößen sollte auch dem jeweiligen kulturellen Kontext Rechnung getragen werden, in dem die für das Sozialkapital wichtigen Verhaltensweisen und Einstellungen gemessen werden (so können z.B. die in einer Erhebung gestellten Fragen hinsichtlich des Vertrauens in den einzelnen Ländern ganz unterschiedlich aufgefasst werden).

Man muss sich jedoch über die Schwierigkeiten im Klaren sein, die die Messung des Sozialkapitals aufwirft. Der Wunsch zur Durchführung derartiger Messungen kann dazu verleiten, Quellen, Funktionen und Ergebnisse miteinander zu verwechseln. Viele der für das Sozialkapital relevanten Aspekte sind impliziter Art und betreffen Relationen, womit sie sich nicht für einfache Messungen und Kodifizierungen eignen. Persönliche Einstellungen (z.B. Vertrauen) oder Verhaltensweisen (z.B. der Beitritt zu einem Verband oder die Teilnahme an Wahlen) sind Ersatzmessgrößen für das Sozialkapital, dürfen aber nicht mit dem eigentlichen Konzept des Sozialkapitals als solchem verwechselt werden. Die Bemühungen um Erfassung der Kerndimensionen menschlicher Interaktionen und Beziehungen werden durch den Mangel an geeigneten Datenquellen erschwert. Das wiederum erklärt sich aus der Tatsache, dass kein hinreichend umfassender Fragenkatalog für die Erhebungen vorhanden ist und letztere nicht wirklich zur Messung von Sozialkapital konzipiert wurden. Es ist daher schwierig, an internationale Datenquellen zum Thema Sozialkapital heranzukommen.

Die Weltbank hat anhand von speziell hierfür konzipierten Erhebungen bzw. Erhebungsmodulen Instrumente für die Messung des Sozialkapitals ,von unten“ entwickelt. Narayan und Pritchett (1998) erstellten beispielsweise eine 
Eine inverse Messgröße für das Sozialkapital sind gesellschaftliche Funktionsstörungen ...

Messgröße des Sozialkapitals im ländlichen Tansania unter Verwendung von Daten der Erhebung über Sozialkapital und Armut in Tansania (SCPS Social Capital and Poverty Survey). In dieser auf breiter Basis durchgeführten Erhebung wurden die Personen über Ausmaß und Merkmale ihrer Vereinsaktivitäten wie auch über ihr Vertrauen in verschiedene Institutionen und Persönlichkeiten befragt. Eine Reihe neuer Erhebungen bzw. Erhebungsmodule wird derzeit auch im Vereinigten Königreich und in Australien entwickelt (vgl. Cox und Macdonald, 2000, sowie Schuller et al., 2001).

Eine Methode zur Messung von Veränderungen des Sozialkapitals sind Messungen der gesellschaftlichen Funktionsstörungen oder der fehlenden sozialen Zusammenarbeit. Fukuyama misst diese Veränderungen anhand der Entwicklung von Kriminalität, familiärer Zerrüttung und Vertrauen. Eng mit dem Anstieg der Kriminalitätsraten verbunden sind wachsende ,soziale Devianzen“ (zunehmend unhöfliche und grobe Umgangsformen, Mangel an Bürgersinn usw.). Bei diesem Ansatz liegt der Schwerpunkt auf langfristigen Veränderungen von Sitten, Gebräuchen und Wertmaßstäben als Indikator für Veränderungen des Sozialkapitals. Jedoch ist bei der Verwendung von Indikatoren für gesellschaftliche Funktionsstörungen zur Messung der Veränderungen beim Sozialkapital insofern Vorsicht geboten, als nicht das gesamte Spektrum der den Funktionsstörungen zugrunde liegenden Faktoren bekannt ist und das Sozialkapital nur einen der potentiellen Einflussfaktoren darstellt. Außerdem besteht bei derartigen Konzepten die Gefahr, dass Ursache und Wirkung verwechselt werden.

Bei verschiedenen Erhebungsreihen der World Values Study (1981, 1991 und 1996) ${ }^{10}$ wurde die Vertrauensbereitschaft der Befragten getestet. Ihnen wurde folgende Frage gestellt: „Kann man Ihrer Meinung nach den meisten Menschen generell vertrauen, oder würden Sie sagen, dass man im Umgang mit Menschen gar nicht vorsichtig genug sein kann?" Aus den Antworten geht hervor, dass das Vertrauensniveau in den OECD-Mitgliedsländern sehr unterschiedlich ist (vgl. Tabelle 3.1), wobei die Differenzen zwischen den Ländern im Zeitablauf relativ konstant sind ${ }^{11}$ und auch die jeweiligen Nachbarländer im Trend ähnliche Ergebnisse aufweisen, was zeigt, wie stark das Vertrauen kulturell und regional geprägt ist.

Bei der Interpretation von Vertrauensmessungen ist Vorsicht geboten.

Ein Problem im Zusammenhang mit dieser Art von Erhebungsfragen besteht darin, dass nicht klar ist, ob die Befragten die Bedeutung der Begriffe
„Vertrauen“ oder „die meisten Menschen“ in den verschiedenen Ländern, Kulturen und Perioden auf die gleiche Weise interpretieren. So muss z.B. eine Unterscheidung vorgenommen werden zwischen der Bereitschaft einer Person, anderen Menschen generell und unabhängig vom Kontext zu vertrauen, und der Bereitschaft, Bekannten im Gegensatz zu allgemein gesehen ,,anderen“ Menschen zu vertrauen (diese Frage verweist auf die weiter oben vorgenommene Unterscheidung zwischen dem Vertrauen unter Freunden und Bekannten auf der einen und Fremden gegenüber auf der anderen Seite). Das spezifische Umfeld, der jeweilige Rahmen und die individuelle Situation spielen hierbei eine entscheidende Rolle. In Ländern wie Frankreich, Italien und Spanien, wo das Vertrauen Fremden gegenüber im Vergleich zu anderen OECD-Ländern offensichtlich schwach ausgebildet ist, geben die Befragten für ihren unmittelbaren Familienkreis ein hohes Vertrauensniveau an (gemäß der World Values Study - vgl. Galland, 1999). Obwohl zwischen Vertrauen und bürgergesellschaftlichem Engagement eine Korrelation besteht, stellt Galland zwischen ein- 
Tabelle 3.1 Eine Messgröße des Vertrauens (World Values Study), 1995-1996

Prozentualer Anteil der Personen, denen zufolge man den meisten Menschen vertrauen kann

\begin{tabular}{lllr}
\hline OECD-Mitgliedsländer & & & 35.3 \\
Norwegen & 65.3 & Italien* & 33.2 \\
Schweden & 59.7 & Belgien* & 31.8 \\
Dänemark* & 57.7 & Österreich* & 31.0 \\
Niederlande* & 55.8 & Vereinigtes Königreich & 30.3 \\
Kanada* & 52.4 & Korea & 30.3 \\
Finnland & 47.6 & Tschechische Republik* & 29.8 \\
Irland* & 47.4 & Spanien & 28.1 \\
Japan & 46.0 & Mexiko & 24.6 \\
Island* & 43.6 & Ungarn* & 22.8 \\
Deutschland & 41.8 & Frankreich* & 21.4 \\
Schweiz & 41.0 & Portugal* & 6.5 \\
Australien & 39.9 & Türkei & 18.2 \\
Vereinigte Staaten & 35.6 & & 2.5 \\
Ausgewählte Nicht-OECD-Länder & & & \\
Indien & 37.9 & Südafrika & \\
Chile & 21.9 & Argentinien & \\
Nigeria & 19.2 & Brasilien & \\
* Angaben von 1990-1991. & & \\
Anmerkung: In der Erhebung wurde folgende Frage gestellt: „Kann man Ihrer Meinung nach den meisten Menschen generell vertrauen, \\
Quelle: World Vaulues Study; Knack und Keefer (1997).
\end{tabular}

Vertrauen und bürgergesellschaftliches Engagement sind wahrscheinlich miteinander verknüpft. zelnen Gruppen der Gesellschaft große Unterschiede in Bezug auf die Art und Weise fest, wie die Menschen ihre Wahl in Bezug auf die sozialen Netze und die gesellschaftlichen Beziehungen treffen. Ein hohes Maß an Vertrauen in einem Bereich kann mit einem sehr begrenzten Engagement bzw. geringem Vertrauen in einem anderen einhergehen. Galland kommt zu der Schlussfolgerung, dass weder allgemeine Messgrößen des Vertrauens noch des bürgergesellschaftlichen Engagements verlässliche Indikatoren für die Qualität der sozialen Beziehungen oder ihre Interaktion auf Makroebene sind. Aus diesem Grund ist bei der Interpretation der Ergebnisse der World Values Study Vorsicht geboten.

Knack und Keefer (1997) berichten ferner über ein Experiment, bei dem zahlreiche Portemonnaies mit einem Inhalt von 50 \$ in einer Reihe von Städten bewusst „verloren“ wurden. Zwischen dem Prozentsatz der „verlorenen“ Portemonnaies, die in den einzelnen Ländern dem Besitzer zurückgegeben wurden, und der Messgröße des Vertrauens bestand eine Korrelation von 0,67, was einen groben Anhaltspunkt dafür liefert, dass Menschen in Ländern mit hohen Vertrauensindexwerten tatsächlich vertrauenswürdiger sind ${ }^{12}$. Knack und Keefer erstellten ferner einen zweiten Index, mit dem die Stärke der Normen betreffend bürgerliche Zusammenarbeit und Vertrauenswürdigkeit erfasst werden sollte. Dieser Index errechnete sich aus dem Durchschnitt der Antworten auf fünf Fragen, die die Einstellung der Befragten zu bestimmten Verhaltensweisen - wie das Erschwindeln von Leistungen oder das Schwarzfahren in öffentlichen Verkehrsmitteln - betrafen. Der Index zeigte verhältnismäßig geringe Unterschiede zwischen den einzelnen OECD-Ländern. Putman (2000a) belegt, dass in den Vereinigten Staaten zwischen Vertrauen und anderen Messgrößen des Sozialkapitals in Verbindung mit bürgergesellschaftlichem Engage- 
ment und sozialen Kontakten eine sehr starke Korrelation besteht. In Ermangelung eines breiteren und umfassenderen Katalogs von Indikatoren legt dies den Schluss nahe, dass das Vertrauen eine annehmbare Ersatzvariable für das Sozialkapital darstellen dürfte.

\subsection{Die Quellen des Sozialkapitals}

Das Sozialkapital wird von gesellschaftlichen Gruppen gebildet, die von der Familie bis zur Nation reichen.

Fundament des Sozialkapitals ist die Familie.
Sozialkapital wird auf der „Ebene“ von Familien, Gruppen, Unternehmen sowie nationalen oder subnationalen Verwaltungseinheiten und sonstigen Institutionen gebildet. Das Konzept des Sozialkapitals wird in der Regel mit Beziehungen innerhalb der Zivilgesellschaft assoziiert. In Wirklichkeit spielen Vertrauensbeziehungen und Netzwerke aber auch in staatlichen Organisationen und Einrichtungen eine Rolle. Das Sozialkapital ist in Normen und Institutionen eingebettet, die auch öffentliche und rechtliche Körperschaften umfassen. Als Analyseobjekt können auch verschiedene durch Geschlecht, Beruf, Sprache oder ethnische Zugehörigkeit definierte Gruppen innerhalb der Zivilgesellschaft dienen. Für die Entwicklung von Sozialkapital lassen sich folgende Quellen und Dimensionen identifizieren: $a$ ) Familie, b) Schule, c) Gemeinde, d) Unternehmen, e) Zivilgesellschaft, $f$ ) öffentlicher Sektor, g) Geschlecht sowie $h$ ) ethnische Zugehörigkeit. Während der Rolle ehrenamtlicher und bürgerlicher Verbände große Aufmerksamkeit gilt, wurde die Schlüsselrolle von Familie, Schule und Unternehmen bei den jüngsten Debatten und Analysen des Sozialkapitals vergleichsweise vernachlässigt.

Familien schaffen Normen und soziale Verbindungen und bieten ein soziales Netz, von dem die jeweiligen Mitglieder profitieren - das gilt insbesondere für die erweiterten Familien in den so genannten „Familiengesellschaften“ (vgl. Coleman, 1990, und Bourdieu, 1985, 1979). Die innerfamiliären Beziehungen, die auf Gegenseitigkeit sowie auf der Fähigkeit beruhen, den seelischen und materiellen Bedürfnissen der Kinder gerecht zu werden, können auch die Entwicklung von Vertrauen und kameradschaftlichem Verhalten außerhalb des unmittelbaren Familienkreises fördern. Die materielle und seelische Unterstützung, die sich die Familienmitglieder gegenseitig bereitwillig geben, kann dazu führen, dass sie dieses Schema auch auf die Außenwelt projizieren und die gleiche Unterstützung von anderen erwarten. Die Familie ist ferner auch einer der Orte, wo das Lernen beginnt, sowie eine potentielle Inspirationsquelle für den schulischen Erfolg in der formalen Bildung. Angesichts des erheblichen Beitrags, den Bildung zur Entstehung von Sozialkapital leisten kann (siehe weiter unten), kann die Familie auf Grund ihrer bildungsfördernden Funktion einen zusätzlichen positiven Einfluss auf das Sozialkapital ausüben. Coleman misst das Sozialkapital in den Familien anhand folgender Indikatoren: $a$ ) physische Präsenz von Erwachsenen oder Eltern im Haushalt sowie $b$ ) Qualität und Intensität der Aufmerksamkeit, die Erwachsene oder Eltern ihren Kindern schenken. Die beiden letztgenannten Elemente lassen sich auch anhand des zahlenmäßigen Verhältnisses zwischen Eltern und Kindern messen. Generell gilt nach Bereinigung um sonstige Faktoren, je größer die Verwandtschaft und je kleiner die Zahl der Erwachsenen in einem gegebenen Haushalt ist, desto geringer ist das Sozialkapital, das dem Lernenden zur Verfügung steht. Starke Familienbande, d.h. auf Zuneigung gegründete Beziehungen (bonding), können in manchen Fällen der Entstehung weiterreichender „Brückenbeziehungen“ entgegenstehen. 


\section{Auch Schulen und Lerneinrichtungen können zur Entwick- lung von Sozialkapital beitragen ...}

\section{... ebenso wie die Gemeinden ...}

... und die Unternehmen.
Die sozialen Beziehungen, die das Sozialkapital bilden, werden relativ häufig durchtrennt, wenn Familien umziehen (Glaeser, 2001). Unter Annahme sonst gleicher Bedingungen ist das Sozialkapital der Kinder in Alleinerzieherfamilien tendenziell geringer. Je höher die Zahl der Erwachsenen und je geringer die Zahl der Kinder in einem Haushalt sind und je weniger die Familie umzieht, desto höher sind Niveau und Qualität der Aufmerksamkeit, die den einzelnen Kindern im Durchschnitt zuteil wird (McLanahan und Sandefur, 1994, und Hao, 1994). McLanahan und Sandefur geben einen Überblick über die in den USA durchgeführten einschlägigen Untersuchungen und gelangen zu dem Ergebnis, dass Scheidungen, die oft mit einem Rückgang der Einkommen, einer Reduzierung der elterlichen Präsenz wie auch einer Begrenzung des Zugangs zu Gemeinschaftsressourcen verbunden sind, das Wohlbefinden der Kinder wie auch deren Schulleistungen in der Regel beeinträchtigen. Aus einer Reihe von Studien (Biblarz, Raftery und Bucur, 1997, und Simons, 1996) geht hervor, dass das Aufwachsen in einem Alleinerzieherhaushalt oder einer Stieffamilie mit einer Reihe von Nachteilen für die Kinder korreliert, wie niedrige Schulabschlussquoten, höhere Kriminalitätsraten und Drogenmissbrauch bzw. eine größere Wahrscheinlichkeit von Teenagerschwangerschaften. Dieser Punkt wird von Painter und Levine (1999) bestätigt: Sie kommen unter Verwendung von Zeitreihen für die Vereinigten Staaten zu dem Ergebnis, dass die verschiedenen Indikatoren für das Wohlbefinden der Kinder eher die Familienstruktur widerspiegeln als die typischen Merkmale von Risikofamilien. Jedoch können diese Effekte u.U. durch andere Faktoren teilweise oder vollständig wettgemacht werden ${ }^{13}$.

Die Schulen können Werte fördern, die für die soziale Zusammenarbeit von Bedeutung sind, und sie können ferner als Schnittstellen für verschiedene soziale Netzwerke fungieren. Allgemeiner betrachtet können Hochschulen, Erwachsenenbildungsstätten und Berufsverbände die Einrichtung von Netzwerken fördern, die verschiedene Sektoren - Bildung, Unternehmertum und ehrenamtliche Tätigkeiten - übergreifen. Je mehr Unterrichtsmethoden und -gestaltung gemeinsames Lernen und Teamarbeit sowie die Offenheit gegenüber neuen Ideen und kultureller Vielfalt fördern, desto mehr können schulische Einrichtungen zur Entwicklung eines Sozialkapitals beitragen, das zwischen verschiedenen Gruppen der Gesellschaft Brücken schlägt.

Auch die Gemeinden und die Nachbarschaft spielen bei der Sozialkapitalbildung eine Rolle. Die sozialen Interaktionen zwischen Nachbarn, Freunden und Gruppen schaffen Sozialkapital und begründen die Fähigkeit, zusammen auf ein gemeinsames Ziel hinzuarbeiten. Coleman (1990) stellt in seiner Analyse der „Vereinnahmung“ eine Verbindung zwischen der Familie und dem örtlichen Gemeinwesen her; dabei kann das Gemeinwesen Druck auf seine Mitglieder ausüben, indem es bei Nichteinhaltung bestimmter Normen Sanktionen androht. Loury ${ }^{14}$ (1987) sieht im Sozialkapital „lokale öffentliche Güter“, die von der Gemeinschaft produziert werden. Diese lokalen öffentlichen Güter können ganz allgemeiner Natur sein und z.B. den Einfluss Gleichrangiger, Kontakte sowie Freundeskreise umfassen.

In den immer zahlreicher werdenden Veröffentlichungen zum Thema „neue Wirtschaft“ wird zum Teil, so auch bei Drucker, die Bedeutung von Netzwerken, Vertrauen, Partnerschaften und Gemeinschaftsunternehmungen hervorgehoben. Dem liegt der Gedanke zugrunde, dass Innovationen immer mehr auf Zusammenarbeit, raschen Lernprozessen und Netzwerken beruhen. 
Vereine und ehrenamtliche Einrichtungen spielen ebenfalls eine Rolle.
Die innovativen Netzwerke in und zwischen den Sektoren, die Lieferanten, Kunden und Forscher miteinander verbinden, verfügen über ein großes Wachstumspotential. Zwischen industrieller Konzentration und all den Vorteilen der Zusammenarbeit, die Netzwerke bieten, einerseits sowie nationalen Wettbewerbsvorteilen andererseits besteht möglicherweise eine Korrelation (Porter, 1990). So gesehen wird die Fähigkeit zur „Vernetzung“ oder Kommunikation außerhalb der formalen Kanäle immer wichtiger. Die auf formalen Regeln und hierarchischen Machtstrukturen basierenden tayloristischen Organisationsstrukturen stützen sich weniger auf Vernetzungen und Vertrauen als die postfordistischen Organisationsformen, in denen Autorität und Managementverantwortlichkeiten stärker internalisiert sind und wo Vertrauen und Informationsaustausch eine entscheidendere Rolle spielen. Hierfür bedarf es aber eines höheren Qualifikationsniveaus der Mitarbeiter. Wichtig sind informelle Netzwerke, die den Austausch impliziten Wissens fördern. Organisationen, die fähig sind zu „lernen“, wie Wissen und Kompetenzen durch effektivere Formen von Interaktionen, Netzwerken sowie Normen für Vertrauen und Zusammenarbeit sozialisiert werden können, sind wichtige Quellen des Sozialkapitals.

Eine weitere Quelle des Sozialkapitals ist die Zivilgesellschaft. Sie besteht aus „formellen und informellen Gruppen und Organisationen, die unabhängig von Staat und Markt zwecks Förderung diverser Interessen der Gesellschaft in Aktion treten “15. Aus den Beziehungen zwischen Zivilgesellschaft, Staat und Markt entstehen wichtige Synergieeffekte. Ausmaß und Intensität der Beziehungen zwischen den einzelnen Gruppen können dafür bestimmend sein, wie effizient diese Synergien Wohlergehen und Wirtschaftswachstum fördern. In seiner Analyse des Sozialkapitals in verschiedenen Regionen Italiens erklärt Putnam (1993) die zwischen den Regionen bestehenden Unterschiede der wirtschaftlichen und politischen Leistungsfähigkeit mit dem im Laufe der Zeit im Rahmen diverser Vereinigungsformen und bürgerlicher Traditionen erworbenen Sozialkapital. Putnam hebt die Bedeutung unterschiedlicher Arten von Vereinigungen hervor (wozu Gruppen wie Sportvereine, Genossenschaften, Solidaritätsgemeinschaften, kulturelle Einrichtungen, Gewerkschaften usw. gehören). Diese Gruppen fördern die Bildung von Zuneigungs- und Brückenbeziehungen, deren positive Effekte auch eine Steigerung der Effizienz politischer Institutionen umfassen. Für Putnam schaffen die Interaktionen zwischen den Mitgliedern dieser Gruppen und Organisationen mithin horizontale Netzwerke bürgergesellschaftlichen Engagements, die es den Teilnehmern erleichtern, gemeinsam in einer Weise zu agieren, die zur Verbesserung von Produktivität und Wohlergehen der Gemeinschaft insgesamt beiträgt. Kurz, ehrenamtliche Einrichtungen und Netzwerke können bei der Förderung von Vertrauen und bürgergesellschaftlichem Engagement eine ähnliche Rolle spielen wie die Schulen, was auch mit Vorteilen für andere Bereiche der Gesellschaft, wie Staat und Wirtschaft, verbunden ist.

Eine verantwortungsvolle Staatsverwaltung, die sich für das öffentliche Wohlergehen, Rechenschaftspflicht und Transparenz einsetzt, schafft eine solide Grundlage für Vertrauen und soziale Integration, die ihrerseits wiederum das Sozialkapital stärken können. Die politischen, institutionellen und rechtlichen Rahmenbedingungen eines Landes ${ }^{16}$ können als Fundament der Netzwerke und Normen für soziale Zusammenarbeit dienen. Diese beiden Kategorien von Strukturen können sich bei der Förderung des Wohlergehens gegenseitig ergänzen und verstärken. So sorgt das Sozialkapital nicht nur für ein besseres öffentliches Management und effizientere politische Institutionen, sondern 
Das Geschlecht ist ein entscheidender Bestimmungsfaktor für den Zugang zu Sozialkapital ...

... wie auch die ethnische Zugehörigkeit.

In einigen Ländern machen sich Anzeichen für ein Schwinden des bürgergesellschaftlichen Engagements bemerkbar, in anderen hingegen nicht. letztere können darüber hinaus auch auf Gemeinschaftsebene existierende Netzwerke ergänzen, statt diese zu ersetzen, und das Vertrauen stärken.

Das Ausmaß von Vertrauen und sozialem Engagement wie auch die Formen dieses Engagements (formell, informell) können zwischen Männern und Frauen ganz unterschiedlich sein, da diese sich in verschiedenartigen sozialen Netzen bewegen und unterschiedlichen Zugang zu Informationen haben. Die Netzwerke der Männer können zuweilen formell sein, da Männer häufiger einer formellen Beschäftigung nachgehen, während die der Frauen in der Tendenz eher informell und stärker auf die Familie ausgerichtet sind (Moore, 1990). Die Vertrauensfähigkeit der Kinder wurzelt vor allem in der Mutter-KindBeziehung - sehr wahrscheinlich deshalb, weil es in vielen Fällen die Mütter sind, die sich hauptsächlich um die Kinder kümmern -, obwohl die Qualität der Vater-Kind-Beziehung ebenfalls von Bedeutung ist (Amato, 1998, S. 247). Sozialkapital kann auch durch die aktive Beteiligung der Frauen an bürgergesellschaftlichen Aktivitäten, am Arbeitsmarkt und an der Politik aufgebaut werden. Geschlechtsspezifische Diskriminierungen können daher negative Folgen für den Aufbau bestimmter Formen von Sozialkapital haben ${ }^{17}$.

Ethnische Bande sind ein weiteres Beispiel dafür, wie sich Menschen mit gemeinsamen Wertmaßstäben und einer gemeinsamen Kultur zum Nutzen aller zusammenschließen können. Wie Portes und Sensenbrenner (1993) feststellten, sind Elemente wie Gegenseitigkeit, Solidaritäts- und Vertrauenspflicht (d.h. die Existenz sozialer Verpflichtungen) Kernmerkmale der Relation zwischen ethnischer Herkunft und Sozialkapital. Für Zuwanderergruppen ist die ethnische Herkunft zum Zeitpunkt der Ankunft in ihrem neuen Land eine „Quelle von Vorteilen, die die Anpassung erleichtern"; so stützen sie sich auf das Sozialkapital, um Rat und Orientierungshilfen in den verschiedensten Bereichen zu erhalten. Beispielsweise sind ethnische Gruppen Finanz- und Humankapitalquellen für jene, die ein eigenes Unternehmen gründen wollen und die dank der engen Beziehungen innerhalb der Gruppe Kredite bzw. Zuschüsse wie auch Arbeitskräfte zu günstigen Bedingungen bekommen können (Geertz, 1962). Sie können häufig auch wertvolle Informationen über den lokalen Arbeitsmarkt liefern. Ferner bieten ethnische Gruppen ein soziales Netz, indem sie in schwierigen Zeiten materielle und finanzielle Bedürfnisse decken und Kinder sowie alte Menschen betreuen (Abrams und Bulmer, 1986). Natürlich kann die ethnische Zugehörigkeit gleichzeitig auch dazu dienen, zwischen bestimmten Menschen Bindungen herzustellen, von denen andere ausgeschlossen sind.

\subsection{Unterliegen Vertrauen und bürgergesellschaftliches Engagement im Laufe der Zeit einem Wandel?}

Veränderungen beim Niveau des Sozialkapitals spiegeln längerfristige Entwicklungen der Normen, Wertmaßstäbe und Strukturen der sozialen Interaktionen wider. Putman (2000a) hat auf einen Rückgang des sozialen und bürgergesellschaftlichen Engagements in den Vereinigten Staaten hingewiesen. Der dort zu beobachtende Abwärtstrend findet sich in anderen Ländern nicht in demselben Ausmaß wieder. Im Vereinigten Königreich und in Schweden ist die Mitgliederzahl in verschiedenen bürgergesellschaftlichen Organisationen sowohl in absoluter Rechnung als auch im Verhältnis zur Gesamtbevölkerung gestiegen. In einigen Ländern (namentlich Australien) hat sich die Form des 
Außer in Australien und den Vereinigten Staaten gibt es kaum Belege für ein rückläufiges soziales Engagement.

\section{Allerdings verlieren einige traditionelle bürgergesellschaftliche Vereinigungen offensichtlich an Bedeutung ...}

\section{... und es kommen neue Formen des Engagements auf, die sich auf enger begrenzte Anliegen konzentrieren.}

bürgergesellschaftlichen Engagements gewandelt; so ist dieses Engagement heute individualistischer und zeitlich befristeter als früher. Gewisse Länder weisen je nach Alterskohorten Unterschiede in Bezug auf das Niveau von Vertrauen und bürgergesellschaftlichem Engagement auf.

Während die traditionellen, lokal verankerten Formen der sozialen Beziehungen (Familien, Nachbarschaft sowie herkömmliche Massenorganisationen) in vielen OECD-Ländern zahlenmäßig zurückzugehen scheinen, kommen neue Formen der sozialen Bindungen auf, die oft weniger eng, unbeständiger und stärker vom Eigeninteresse diktiert sind. Die Frage, ob diese neuen Formen der sozialen Beziehungen ausreichen werden, um die alten Formen zu ersetzen, lässt sich bisher noch nicht beantworten. Trends und Muster des bürgergesellschaftlichen Engagements, der Beteiligung an Wahlen, der Mitgliedschaft in verschiedenen Arten von Organisationen, des Vertrauensniveaus wie auch der informellen Sozialisierung sind allesamt Faktoren, die wichtige Rückschlüsse auf die in den OECD-Ländern neu entstehenden Trends gestatten. Nachstehend wird ein kurzer Überblick über die Situation in ausgewählten OECD-Ländern gegeben (Australien, Deutschland, Frankreich, Japan, Niederlande, Schweden, Vereinigtes Königreich und Vereinigte Staaten). Nähere Einzelheiten hierzu finden sich in Anhang E.

Die verfügbaren Informationen unterstreichen folgende Aspekte:

- stabile bzw. wachsende Mitgliedschaft in bürgergesellschaftlichen Organisationen (außer in Australien und den Vereinigten Staaten);

- gleichbleibendes Niveau bzw. Zunahme der ehrenamtlichen Tätigkeiten in den meisten Ländern;

- widersprüchliche Belege, was das rückläufige Engagement junger Menschen in Verbänden und ehrenamtlichen Einrichtungen angeht;

- widersprüchliche Belege, was den Rückgang der informellen sozialen Kontakte angeht (allerdings lassen Qualität und Vollständigkeit der Daten zu wünschen übrig);

- widersprüchliche Belege, was die Abnahme von politischem Interesse und Aktivismus angeht;

- Anzeichen für einen Mitgliederschwund in gewissen traditionellen Vereinigungen, namentlich Gewerkschaften, Kirchen und Frauenbewegungen;

- gewisse Anzeichen für einen Rückgang des zwischenmenschlichen Vertrauens wie auch des Vertrauens in die politischen Institutionen (insbesondere unter den jungen Menschen im Vereinigten Königreich und in den Vereinigten Staaten);

- Entstehung neuer Sozialbewegungen in den letzten Jahrzehnten (zur Verteidigung bestimmter spezifischer Interessen, u.a. im Umweltbereich);

- verstärkte Teilnahme an kulturellen und sportlichen Einrichtungen in den meisten Ländern; 
Das Vertrauen unter den Menschen nimmt $z u$, während das Vertrauen in die öffentlichen Institutionen abnimmt.

\author{
Die Unterschiede \\ zwischen den \\ Generationen sind \\ in den Vereinigten \\ Staaten besonders \\ ausgeprägt.
}

- strukturelle Veränderung der Teilnahme am politischen Leben; Direktkontakte spielen eine geringere, Beziehungen zu Fachleuten und Medien hingegen eine zunehmend wichtige Rolle;

- gewisse Anzeichen für einen Wandel des Engagements zu Gunsten informellerer sozialer Kontakte, die von der Tendenz her in stärkerem Maße individualistischer und temporärer Natur sind, aber nicht unbedingt materialistischer oder ichbezogener sein müssen;

- die neueren Formen der Teilnahme an der Zivilgesellschaft sind enger begrenzt und individualistischer, d.h. möglicherweise weniger auf kollektive oder Gruppeninteressen bzw. -ziele ausgerichtet. Wir erleben derzeit vielleicht eine gewisse Privatisierung des Sozialkapitals.

Mit Hilfe internationaler Daten der World Values Study hat Inglehart (1997) ferner einen Rückgang des Vertrauens in verschiedene Arten von Institutionen, darunter auch in politische und religiöse Hierarchien, festgestellt. Den Befragungen zufolge war das Niveau des Vertrauens in Staat, öffentlichen Dienst, Polizei, Kirchen, Bildungssysteme und Medien in den meisten Ländern zwischen 1981 und 1990 rückläufig. Demgegenüber wurde im gleichen Zeitraum nur eine recht geringe negative Veränderung bzw. sogar ein gewisser Anstieg des zwischenmenschlichen Vertrauens beobachtet. Diese Trendentwicklungen hängen möglicherweise mit dem höheren Bildungsniveau wie auch mit einer Verlagerung der Werte zu Gunsten einer größeren persönlichen Autonomie und einer abnehmenden Autoritätsgläubigkeit zusammen.

\subsection{Worauf sind die Veränderungen beim Vertrauensniveau und beim bürgergesellschaftlichen Engagement zurückzuführen?}

Ist der Rückgang des Sozialkapitals in den Vereinigten Staaten und Australien ein Ausnahmephänomen oder kündigt er vielmehr entsprechende Entwicklungen in anderen Ländern an? Die vorhandenen Belege deuten darauf hin, dass die intergenerativen Effekte in den Vereinigten Staaten besonders stark sind. Die jüngeren Generationen (vor allem die seit den fünfziger Jahren Geborenen) sind in der Regel weniger bereit, Organisationen oder ehrenamtlichen Verbänden beizutreten, zu wählen, sich sozial zu engagieren oder anderen Menschen zu vertrauen. Putnam (2000a) kommt zu der Schlussfolgerung, dass der Effekt generationsübergreifender Unterschiede langfristig besonders stark ist und die Auswirkungen der Zugehörigkeit zu einer gesellschaftlichen Klasse, ethnischen Gruppe oder Religion überwiegt. Zu dieser Schlussfolgerung kommt der Autor unter Verwendung einer Vielzahl von Datenquellen, auf Grund deren differenziert werden kann zwischen den Veränderungen, die sich im Laufe des Lebens vollziehen (das bürgergesellschaftliche Engagement erreicht normalerweise zwischen 40 und 50 Jahren seinen Höhepunkt), den Unterschieden zwischen den einzelnen Alterskohorten zu bestimmten Zeitpunkten (Vergleich der Babyboomgeneration mit älteren Generationen) und schließlich Divergenzen, die mit bestimmten Zeitepochen zusammenhängen (und somit den Effekt einmaliger oder plötzlicher Veränderungen auf sämtliche Kohorten widerspiegeln).

Es ist nicht leicht herauszufinden, warum die Unterschiede zwischen den Generationen so groß sind. Putnam weist darauf hin, dass der Zweite Weltkrieg und seine Folgen zur Entwicklung eines stark ausgeprägten national eingefärbten staatsbürgerlichen Engagements beigetragen haben, dessen Auswirkungen 
In den Wertesystemen vollziehen sich derzeit sogar noch weitreichendere Veränderungen ...

... die zeitweilig Hand in Hand mit einem schwächeren Engagement für Anliegen der Allgemeinheit gehen.

Es entstehen möglicherweise neue Formen des Engagements. sich bei allen Generationen bemerkbar machten, die im Zeitraum 1910-1940 geboren wurden. Die Wiedergeburt des Sozialkapitals, die zu Beginn des 20. Jahrhunderts in den Vereinigten Staaten zu beobachten war (und die auf eine Phase des Rückgangs gegen Ende des 19. Jahrhunderts folgte, welche Parallelen zur gegenwärtigen Entwicklung aufweist), hing mit verschiedenen bürgergesellschaftlichen Erneuerungsbewegungen um die Jahrhundertwende zusammen. Putman wirft in seiner Analyse die Frage auf, ob die analogen Entwicklungsmuster in Australien auf ähnliche Generationeneffekte zurückzuführen sind und weshalb diese in anderen Ländern nicht in demselben Maße zu Tage getreten sind.

In den meisten OECD-Ländern finden derzeit bedeutende Umwälzungen bei Wertesystemen, Normen und sozialen Verhaltensmustern statt. Einige Beobachter (darunter Inglehart, 1997) gehen davon aus, dass diese Veränderungen Teil einer natürlichen Entwicklung sind, die in den modernen Gesellschaften auf mehr persönliche Autonomie, weniger hierarchische Kontrolle bzw. geringere Gruppen- und kulturelle Identität abzielt und bei der in einigen Fällen auch postmaterialistische oder ,postmoderne“ bzw. individualistische Ziele angestrebt werden. Inglehart (1991) und Yankelovich (1981) geben ferner zu bedenken, dass der zunehmende materielle Wohlstand eine der Hauptursachen für soziale Unruhen und Funktionsstörungen im Anfangs- und im mittleren Stadium der Wirtschaftsentwicklung darstellt, während in einem späteren Stadium der wirtschaftlichen Entwicklung dann eine relative wirtschaftliche Sicherheit möglicherweise die Rückkehr zu traditionelleren Werten nach sich zieht. Diese Verlagerungen dürften jedoch eher zu Veränderungen als zu einem Rückgang der sozialen Beziehungen führen.

Das Entstehen neuer Werte, die den Akzent stärker auf Selbstständigkeit als auf Respekt legen, können unerwünschte Nebenwirkungen haben, wie beispielsweise eine Abnahme des Pflichtbewusstseins und des Engagements für die „gemeinsame Sache“. Während der Einzelne früher in Großfamilien, in der Kirche und in anderen sozialen Strukturen Rückhalt fand, haben diese traditionellen Auffangnetze heute nicht mehr dieselbe Bedeutung. Das hat u.a. zur Folge, dass sich der Übergang ins Erwachsenenleben für junge Menschen, die auf der Suche nach ihrer Identität, nach inneren Überzeugungen und nach ihrem Platz in der Gesellschaft sind, heute wahrscheinlich problematischer ist. Nach Auffassung einiger Kommentatoren (z.B. Fukuyama, 1999) schlägt sich die Erosion der gemeinsamen Wertvorstellungen, Identitäten und sozialen Ziele in einer Erosion des Vertrauens und der Bereitschaft zu einem überzeugten bürgergesellschaftlichen Engagement im Dienst des öffentlichen Interesses nieder.

Laut Rothstein (1998) könnte die Entwicklung dahin gehen, dass die Menschen immer weniger den gleichen Wertkodex und Lebensstil teilen, dafür aber stärker an allem interessiert sind und sich sozial stärker engagieren. Auf der Grundlage von Erhebungsdaten für Schweden kommt er zu der Schlussfolgerung, dass der Einzelne heute eher bereit ist, anderen zu helfen und gleichzeitig zu akzeptieren, dass seine Mitmenschen nicht die gleichen Wertvorstellungen wie er selbst haben und sich für anders geartete Anliegen einzusetzen gewillt sind. Offen ist die Frage, ob diese Feststellung auch auf andere Länder übertragbar ist; sie veranschaulicht aber eine der zentralen Herausforderungen, vor die eine solche Veränderung von Wertvorstellungen und Normen die Menschen stellt. In vielen Fällen dürften Bemühungen um eine Wiederbelebung der traditionellen Formen des Engagements wenig sinnvoll 
Zwischen gewohnheitsmäßigem Fernsehen und nachlassendem bürgergesellschaftlichen Engagement besteht ein Zusammenhang ...

... während die Effekte des Internets noch nicht voll zum Tragen kommen.

Die längere Arbeitszeit und die Verstädterung spielen ebenfalls eine Rolle. traditionellen Formen des Engagements wenig sinnvoll sein. Die Anstrengungen sollten sich vielmehr darauf konzentrieren, die Gesellschaften durch ein stärkeres bürgergesellschaftliches und öffentliches Engagement entsprechend den neuen Erwartungen und Einstellungen zu dynamisieren.

Putnam (2000a) findet eine starke Verbindung zwischen der immer größeren Ausbreitung des Fernsehens in den Vereinigten Staaten (bei einer gleichzeitig weniger selektiven Programmauswahl) einerseits und dem rückläufigen sozialen Engagement - unter Berücksichtigung anderer Faktoren wie Abstammung, sozialer Status und Alter - andererseits. Starke intergenerative Effekte scheinen im Verein mit der Zunahme der vor dem Fernseher verbrachten Zeit den weit überwiegenden Teil des Sozialkapitalschwunds in den Vereinigten Staaten seit Mitte der sechziger Jahre zu erklären. De Hart und Dekker (1999) finden einen Zusammenhang zwischen dem Fernsehen und der Abnahme des bürgergesellschaftlichen Engagements in den Niederlanden (womit sie nicht den Rückgang des gesamten Sozialkapitals zu erklären, sondern vielmehr die Frage zu beantworten suchen, warum einige Gruppen stärker engagiert sind als andere). Sie stellten fest, dass Menschen, die sehr viel fernsehen, vergleichsweise weniger Zeit dem Besuch von Freunden und Bekannten, ehrenamtlichen Tätigkeiten, Einladungen bei sich zu Hause, Gesprächen mit anderen Familienmitgliedern, Briefeschreiben oder Telefonieren widmen ${ }^{18}$. Feststellungen wie diese werfen die Frage auf, warum - angesichts der weltweiten Zunahme des Fernsehens - nur in den Vereinigten Staaten und vielleicht noch in Australien ein so deutlicher Rückgang des Sozialkapitals zu beobachten ist.

Da die Internetrevolution noch nicht lange zurückliegt, ist es laut Putnam (2000a, S. 170) schwer, hierin eine der Hauptursachen für die Veränderungen bzw. den Rückgang des Sozialkapitals zu sehen - vor allem in den Vereinigten Staaten, wo der Rückgang bereits 25 Jahre früher eingesetzt hat. In Zukunft könnten die Interneteffekte aber erheblich sein. Wichtig ist an dieser Stelle jedoch der Hinweis darauf, dass die computermediatisierte Kommunikation für den Informationsaustausch, das Einholen von Meinungen und die Erörterungen von Alternativen zwischen physisch weit voneinander entfernten Personen zwar als positiv gewertet worden ist, andererseits aber oft die Erfahrung gemacht wurde, dass der Aufbau von Vertrauensbeziehungen in einem virtuellen Umfeld relativ schwierig ist. In Wirklichkeit scheint die Kommunikation über das Internet die direkten sozialen Interaktionen eher zu ergänzen als zu ersetzen.

Putnam vertritt die Auffassung, dass Faktoren, wie die Verlängerung der Arbeitszeit, die Zunahme von Doppelverdienerhaushalten sowie die Verstädterung, in den Vereinigten Staaten mit zum Rückgang des Sozialkapitals beitragen, aber von geringerer Bedeutung sind als die intergenerativen Unterschiede und das Fernsehen. Die Tatsache, dass Menschen immer häufiger multifunktionale ländliche Gebiete zu Gunsten reiner Wohnviertel in Städten und Vorstädten verlassen, hat den Rückgang des bürgergesellschaftlichen Engagements offenbar verstärkt. Gleichzeitig konnte Putnam aber keine Belege dafür finden, dass die sich wandelnden Strukturen der Frauenerwerbstätigkeit in der abhängig beschäftigten Erwerbsbevölkerung starke Auswirkungen auf die Teilnahme am Gemeinschaftsleben haben. Unter Berücksichtigung von Faktoren wie Bildungsniveau, Familienstand und Geburtsjahr kommt Putnam zu dem Ergebnis, dass in den Vereinigten Staaten in sämtlichen Beschäftigungskategorien (Vollzeit, Teilzeit und Erwerbslosigkeit) Frauen sozial stärker engagiert sind als Männer. Teilzeitbeschäftigte Frauen (insbesondere jene, die ein befriedi- 
Es ist die Vermutung geäußert worden, der Wohlfahrtsstaat verdränge freiwillige Initiativen und Sozialkapital, die Beweislage ist jedoch schwach.

Die zunehmende

Instabilität des

Familienlebens könnte das Sozialkapital geschwächt haben. gendes Gleichgewicht zwischen Berufs- und Familienleben gefunden haben) sind in der Regel sozial stärker engagiert ${ }^{19}$. Verblüffend war in diesem Zusammenhang die Schlussfolgerung, dass die Teilnahme am Gemeinschaftsleben in den Vereinigten Staaten in allen Beschäftigungskategorien zu beobachten ist, selbst unter Frauen, die keiner Erwerbstätigkeit nachgehen, was darauf hindeutet, dass die strukturellen Veränderungen der Frauenbeschäftigung kein Faktor sind, der wesentlich zum Rückgang des Bürgerengagements beigetragen hat. Jedoch dürften in den Vereinigten Staaten sowohl der Zeitmangel als auch die Verlängerung der Arbeitszeit bei Männern wie Frauen eine gewisse Rolle gespielt haben.

Als mögliche Ursache für den Rückgang des Sozialkapitals ist der Wohlfahrtsstaat angeführt worden. Dabei wird argumentiert, dass sich die familiären, assoziativen und zwischenmenschlichen Beziehungen in einem Wohlfahrtsstaat, in dem soziale Verpflichtungen zunehmend vom Staat übernommen werden, abschwächen und dem Einzelnen weniger Anreize zur Teilnahme an ehrenamtlichen Aktivitäten geboten werden (Norton, 1998). Eine mögliche Alternative dazu wäre, dass der Wohlfahrtsstaat, statt ehrenamtliche Tätigkeiten und Initiativen zu verdrängen, die Solidarität sowohl symbolisch als auch praktisch fördert. Dies kann über Sozialschutzprogramme geschehen, die die persönlichen Risiken verringern, wie auch über die Förderung des Erwerbs von Fertigkeiten und Kompetenzen, die den Einzelnen ermutigen und es ihm ermöglichen, sein Potential voll zu entfalten (HRDC, 1999). Die Feststellungen von de Hart und Dekker (1999), Rothstein (1998) sowie Hall (1999) für die Niederlande, Schweden bzw. das Vereinigte Königreich tragen nicht zur Erhärtung der Verdrängungseffekt-Hypothese bei. Diese Autoren kommen zu der Schlussfolgerung, dass das Niveau der ehrenamtlichen Tätigkeiten, der informellen Sozialisierung und der Teilnahme an Gemeinschaftsprojekten in diesen Ländern relativ hoch ist und kaum Belege dafür vorhanden sind, dass dieses Niveau durch die öffentliche Sozialpolitik vermindert bzw. beeinträchtigt worden wäre.

Die in acht europäischen Ländern durchgeführte Erhebung zeigt vor allem, dass zwei der Länder mit der großzügigsten Sozialpolitik - die Niederlande und Schweden - gleichzeitig auch die Länder mit dem größten Volumen an unbezahlter Arbeit in ehrenamtlichen Einrichtungen sind (Gaskin und Davis Smith, 1995 ${ }^{20}$. Als Antwort auf die Frage „Haben Sie im vergangenen Jahr unentgeltliche Arbeiten oder Aktivitäten für oder in einer Organisation durchgeführt, die in keinem Zusammenhang zu Ihrer entlohnten Beschäftigung steht und weder ausschließlich in Ihrem noch im Interesse Ihrer Familie liegt?" gaben 36\% der Schweden ,ja“ an, gegenüber einem Durchschnitt von 27\% in den anderen europäischen Ländern.

Seit der industriellen Revolution hat die Rolle der Kern- oder Großfamilie sowohl als wirtschaftliche Produktionseinheit als auch als Bildungsstätte an Bedeutung verloren, was möglicherweise auch gewisse Familiennetze geschwächt hat. In jüngerer Zeit beobachtete Veränderungen der Struktur und Stabilität der Familien deuten darauf hin, dass die Menschen heutzutage für die Beschaffung von Finanzmitteln, die Beaufsichtigung der Kinder und deren Erziehung eher auf unpersönliche Beziehungen zurückgreifen. Jedoch kann die Großfamilie mit ihrem kulturellen Kapital noch immer ein wichtiges soziales Sicherheitsnetz bieten, indem sie in schwierigen Zeiten materielle und finanzielle Bedürfnisse erfüllt und einen Beitrag zur Betreuung von Kindern und älteren Menschen leistet (Abrams und Bulmer, 1986). 
Nach Projektionen, die auf Daten der jüngsten Zeit beruhen (The State of America's Children, 1998, Yearbook Children's Defense Fund), lebt jedes zweite Kind während seiner Kindheit irgendwann einmal in einem Alleinerzieherhaushalt, jedes dritte Kind wird unehelich geboren, jedes vierte Kind wächst mit nur einem Elternteil auf, jedes achte Kind wird von einer Teenagermutter geboren und jedes 25. Kind wächst ganz ohne seine natürlichen Eltern auf. Für Fukuyama sind das Auseinanderbrechen der Familienstruktur, das Fehlen eines oder beider Elternteile wie auch der wachsende Individualismus Faktoren, die das Vertrauen untergraben und zunehmende soziale Funktionsstörungen verursachen. Auch wenn gegenwärtig noch keine Klarheit über die längerfristigen Auswirkungen der beschriebenen Veränderungsprozesse auf die Förderung von Vertrauen und bürgergesellschaftlichem Engagement besteht, ist dieser Wandel doch auf jeden Fall mit wichtigen Konsequenzen für die Sozialpolitik verbunden, da im Zuge der sich lockernden familiären Beziehungen und der Alterung der Bevölkerung die Gefahr der sozialen Isolation bedürftiger Menschen wächst.

\subsection{Welche Auswirkung hat das Sozialkapital auf das Wohlergehen?}

Engere soziale Beziehungen bieten eine Vielzahl von Vorteilen.

Zwischen Sozialkapital und guter Gesundheit besteht eine Korrelation ...
Ebenso wie das Humankapital bietet auch das Sozialkapital dem Einzelnen und der Gesellschaft große Vorteile. Einige dieser Nutzeffekte, wie die Steigerung der Produktivität, sind unmittelbar ökonomischer Natur, und konkrete Belege für diese Effekte werden in Abschnitt 3.9 untersucht. Im vorliegenden Abschnitt sollen hingegen die Effekte des Sozialkapitals auf andere Aspekte des Wohlergehens betrachtet werden.

Eine Reihe in verschiedenen Ländern durchgeführter Studien hat - unter Berücksichtigung des jeweiligen gesundheitlichen Ausgangszustands - gezeigt, dass zwischen der Intensität der sozialen Beziehungen einerseits - die daran gemessen wird, wie eng die Beziehungen sind, die der Einzelne zu Verwandten, Freunden und Bekannten aufbaut und unterhält - und der Lebenserwartung andererseits ein Zusammenhang besteht ${ }^{21}$. Putnam $(2000 a)$ bietet einen Überblick über eine Vielzahl empirischer Studien, die alle zu der Schlussfolgerung kommen, dass zwischen dem Sozialkapital (das vor allem an der Existenz sozialer Netze gemessen wird) und Bildung, Wohlergehen der Kinder, Kriminalität, Vitalität des jeweiligen Viertels (gemessen z.B. an den Wiederverkaufspreisen der Wohnungen), (physischer und psychischer) Gesundheit, Glück und demokratische Regierungsformen ein positiver Zusammenhang besteht. Er stellt ferner eine starke und bedeutende Korrelation zwischen den Messgrößen des Sozialkapitals in den verschiedenen US-Bundesstaaten und einer Gesamtmessgröße für das Wohlbefinden der Kinder fest, wobei Merkmale wie Abstammung, Einkommen und Niveau der schulischen Erstausbildung berücksichtigt wurden.

Am deutlichsten zeigen sich die positiven Auswirkungen sozialer Bedingungen wahrscheinlich im Bereich der Gesundheit. Bereits im 19. Jahrhundert fand der Soziologe Emile Durkheim eine enge Verknüpfung zwischen der Selbstmordhäufigkeit und dem Grad der gesellschaftlichen Integration der betreffenden Person. Er fand heraus, dass die Suizidrate in Zeiten des raschen sozialen Wandels steigt. Zurückgeführt wurde dieses Phänomen auf das Aus- 
... während soziale Isolation die Menschen unglücklich und krank machen kann. einanderbrechen des gesellschaftlichen Gefüges und die Schwächung der sozialen Beziehungen. Putnam (2000a) zitiert die Ergebnisse zahlreicher neuer Studien, die auf eine Verknüpfung zwischen sozialen Bindungen auf der einen und guter Gesundheit sowie persönlichem Wohlergehen auf der anderen Seite hindeuten (unter Berücksichtigung der individuellen Merkmale sozialer, ethnischer und demographischer Art). Auch die individuelle Lebensdauer scheint durch das Ausmaß der sozialen Kontakte beeinflusst zu werden. Putnam führt für diese Zusammenhänge zwei mögliche Gründe an: a) Die sozialen Netzwerke sorgen ganz konkret für Unterstützung und Betreuung, was den psychischen und physischen Stress reduzieren hilft. b) Das Sozialkapital löst möglicherweise einen physiologischen Mechanismus aus, der das Immunsystem des Einzelnen stimuliert, so dass der Organismus besser in der Lage ist, Krankheiten abzuwehren und Stress zu ertragen.

Die Forschungsarbeiten über die Effekte des Sozialkapitals auf den Gesundheitszustand zeigen, dass soziale Isolation in der Regel Krankheiten vorausgeht, was die These erhärtet, dass soziale Isolation eher Ursache als Folge von Krankheit ist. Die psychologische Fachliteratur aus mehr als drei Jahrzehnten bestätigt den Zusammenhang zwischen sozialen Unterstützungsbeziehungen und geistiger Gesundheit (z.B. Brown und Harris, 1978). Auch Putnam (2000a) gibt einen Überblick über die vorhandenen Belege bezüglich der Auswirkungen des Sozialkapitals auf Glück und Wohlergehen (gemäß den subjektiven Aussagen der Betroffenen). Auch hier ergibt sich wiederum - unter Bereinigung um sonstige Faktoren - ein positiver Zusammenhang. Putnam stellt fest, dass „... die Befragten in den verschiedenen Untersuchungen immer wieder selbst berichten, dass gute familiäre, freundschaftliche oder Liebesbeziehungen für ihr Glück sehr viel wichtiger sind als Ruhm oder Geld“ (S. 332). Bei älteren Menschen, die allein leben und weder Freunde noch Verwandte haben, besteht unter Annahme sonst gleicher Bedingungen ein vergleichsweise größeres Risiko, dass sie Krankheiten wie Demenz oder Alzheimer entwickeln. Eine mit 1200 Personen über drei Jahre vom Forschungszentrum für Gerontologie in Stockholm durchgeführte Erhebung hat ergeben, dass ein weitgespanntes soziales Netz einen der Faktoren bildet, die vor Demenz schützen (Fratiglioni et al., 2000). Es konnte gezeigt werden, welche Bedeutung zufriedenstellenden Kontakten mit anderen Menschen, insbesondere mit der unmittelbaren Familie und den Kindern, zukommt. Derartige Kontakte scheinen auch dann vor Demenz zu schützen, wenn sie relativ selten sind.

Rose (2000) wies nach, dass in Russland die Beziehungen zu anderen Menschen, denen man vertrauen und auf die man sich verlassen kann, wenn man Hilfe oder Ratschläge für sein persönliches Leben benötigt, einen Großteil der zwischen den Menschen bestehenden Unterschiede in Bezug auf deren körperliche und seelische Verfassung erklären. Der Effekt des Sozialkapitals war in diesen Untersuchungen etwa so groß wie der des Familieneinkommens, jedoch größer als der des Bildungsabschlusses. Rose fand Belege für starke informelle Netzwerke zwischen Freunden, Verwandten und sonstigen, sich nahe stehenden Gruppen auf der einen Seite und parallel dazu für die Existenz eines distanzierteren politischen und sozialen Gefüges auf der anderen Seite, das durch einen geringeren Grad an Vertrauen gekennzeichnet ist. Er schloss daraus, dass sich die Menschen zur Bewältigung ihrer Probleme stärker auf das informelle Sozialkapital als auf die formellen staatlichen Einrichtungen verlassen. 
Das Sozialkapital verbessert das Wohlergehen der Kinder ...

... reduziert die Fälle von Kindesmissbrauch ...

... und erleichtert
den Übergang ins
Erwachsenenleben.

Zwischen Sozialkapital und Verringerung der Kriminalität besteht ebenfalls ein Zusammenhang
Putnam (2000a) liefert Belege dafür, dass das Sozialkapital von der Breitenwirkung und Tiefe der Effekte auf das Leben der Kinder her gleich nach der Armut an zweiter Stelle rangiert ${ }^{22}$. Während Armut mit erhöhten Teenagerschwangerschafts- und Mortalitätsraten sowie mit vermehrtem Müßiggang einhergeht, hat das gemeinschaftliche Engagement genau den gegenteiligen Effekt. Demgegenüber hat das Bildungsniveau der Erwachsenenbevölkerung nach Bereinigung um Faktoren wie Armut, Sozialkapital und demographische Merkmale einen sehr viel geringeren Effekt auf das Wohlergehen der Kinder. Jedoch dürften diese Ergebnisse, wenn man den eindeutig identifizierten Wirkungseinfluss der Bildung auf Armut und Sozialkapital bedenkt, dem interaktiven bzw. kombinierten Effekt des Humankapitals, das auf vielerlei Weise, darunter auch über das Sozialkapital und den sozioökonomischen Status, wirksam wird, nicht hinreichend Rechnung tragen.

Psychologische Gutachten zeigen, dass die Kindesmissbrauchsraten in Wohnvierteln mit niedrigerem sozialen Zusammenhalt höher sind (Korbin und Coulton, 1997). Garbarino und Sherman (1980) untersuchten zwei Viertel mit ähnlichem Einkommensniveau und ähnlicher Verteilung von Haushalten mit erwerbstätigen Frauen und Alleinerzieherhaushalten. Die Bewohner von Vierteln mit relativ größerem Kindesmissbrauchsrisiko waren zurückhaltender, wenn es darum ging, Nachbarn um Hilfe zu bitten, und die Eltern weniger bereit, gegenseitig auf ihre Kinder aufzupassen oder die Kinder mit anderen spielen zu lassen. Bei Kindern aus Vierteln mit geringerem Risiko war die Wahrscheinlichkeit im Vergleich zu ihren Kameraden aus Hochrisikovierteln dreimal so groß, dass sie nach der Schule einen Elternteil zu Hause vorfinden.

Runyan und andere haben außerdem festgestellt, dass die sozialen Kontakte der Mutter von zentraler Bedeutung sind, wenn es darum geht, die Kinder im späteren Leben erfolgreich vor verhaltensmäßigen und emotionalen Problemen zu bewahren. Soziale Beziehungen können sowohl soziale Unterstützungsnetze für Mütter wie auch aufgeschlossene und hilfsbereite Nachbarn im näheren und weiteren Umfeld umfassen. Die Autoren kommen zu folgender Schlussfolgerung: „Das Sozialkapital der Eltern ... kommt deren Kindern ebenso zugute, wie es beim Finanz- und Humankapital der Fall ist" (Runyan et al., 1998). In Familien mit relativ engen Bindungen ist die Wahrscheinlichkeit größer, „dass die familiären Wertvorstellungen geachtet und übernommen werden". Wenn den Kindern innerhalb der Familie Vertrauen beigebracht wird, kann dies den jungen Menschen den Übergang ins Erwachsenendasein und ihre aktive Teilnahme am Leben der Gesellschaft erleichtern (Teachman, Paasch und Carver, 1999, sowie Darling und Steinberg, 1997).

Das Sozialkapital spielt eine wichtige Rolle, indem es nicht nur antisoziale oder kriminelle Verhaltensweisen entmutigt, sondern auch, indem es das in der Gemeinschaft vorhandene Energiepotential valorisiert und in die richtigen Bahnen lenkt. Aus einer Synthese der Ergebnisse der World Values Study und der International Crime Victim Survey ${ }^{23}$ (ICVS) geht hervor, dass die zwischen den Ländern bestehenden Unterschiede in Bezug auf die Zahl der Opfer von Straftaten auf eine Reihe sozialer Normen und Wertvorstellungen zurückzuführen sein dürften, die Teil des Sozialkapitals sind. Halpern (2001) verwendet für 18 Länder ICVS-Daten, die einen Zusammenhang herstellen zwischen bestimmten Wertvorstellungen und der Häufigkeit von Straftaten, wobei diese Daten um Faktoren wie Ungleichheit, Pro-Kopf-BIP und 
Vertrauensklima bereinigt sind. Er fand erwartungsgemäß eine deutliche Korrelation zwischen Kriminalität und „egoistischen“ Verhaltensweisen (wozu z.B. die Neigung gehört, gefundenes Geld zu behalten, zu betrügen, zu lügen und in öffentlichen Verkehrsmitteln „schwarz“ zu fahren). Diese Verhaltensweisen waren besonders verbreitet unter jungen Menschen, Männern und Stadtbewohnern. Zwei Drittel der nationalen Unterschiede in Bezug auf die Verbrechenshäufigkeit konnten auf Abweichungen der länderspezifischen Variablen für Eigennützigkeit, wirtschaftliche Ungleichheit und soziales Vertrauen erklärt werden ${ }^{24}$. Halpern fasst seine Ergebnisse zusammen, indem er die wirtschaftliche Ungleichheit als „Motiv“, das soziales Vertrauen als „Gelegenheit“ und „Eigennutzdenken“ als Instrument zur Ausführung von Straftaten bezeichnet. Auch wenn sich diese Ergebnisse auf zu einem bestimmten Zeitpunkt erhobene nationale Daten beziehen und nicht als Beweis für Kausalbeziehungen gewertet werden können, deuten sie dennoch insgesamt gesehen auf eine Reihe potentiell wichtiger Zusammenhänge zwischen bestimmten Aspekten des Sozialkapitals und der Verbrechenshäufigkeit hin. Kawachi et al. (1997) äußern ferner die Vermutung, dass ein zentrales ursächliches Element der Beziehungen zwischen Gewaltverbrechen, sozialem Misstrauen und Ungleichheit in mangelnder Selbstachtung, Verlust an Würde und niedrigem sozialen Status besteht. Wo Selbstachtung, Menschenwürde und sozialer Status durch Armut und Ausgrenzung untergraben werden, schwächt dies auch das Vertrauen und die sozialen Bindungen, was sich negativ auf Gesundheit und Verbrechensneigung auswirkt.

Beobachtungen in den Vereinigten Staaten zeigen, dass selbst bei Bereinigung um Armut und sonstige Faktoren, die kriminelles Verhalten fördern können, Gemeinden, die durch a) Anonymität bzw. nur begrenzten Kontakt zwischen den Mitbewohnern, $b$ ) Präsenz unbeaufsichtigter Gruppen von Jugendlichen sowie $c$ ) ein niedriges Niveau des lokalen bürgergesellschaftlichen Engagements gekennzeichnet sind, verstärkt der Gefahr von Kriminalität und Gewalt ausgesetzt sind (Sampson und Morenoff, 1997, sowie Sampson, 1995). Putnam (2000a) vertritt die Auffassung, dass in den Vereinigten Staaten die Verschlechterung des Sozialkapitals in den einzelnen Vierteln, das seinen Ausdruck findet in einer sozialen Überwachungsfunktion sowie in Sozialisierung, Betreuung und Organisation von Gruppen, ein wichtiger Bestimmungsfaktor für den Niedergang der Innenstädte ist. Sampson, Raudenbush und Earls (1997) zeigten mit Hilfe umfassender Erhebungsdaten für Wohnviertel in Chicago, dass gegenseitiges Vertrauen und nachbarschaftlicher Altruismus eine Schlüsselrolle bei der Erklärung der zwischen den einzelnen Vierteln bestehenden Unterschiede in Bezug auf die Verbrechenshäufigkeit spielen (unter Berücksichtigung der jeweiligen wirtschaftlichen und sozialen Merkmale des Viertels). Ihre Analyse, die die Beobachtungen Putnams über die Auswirkungen des informellen Sozialkapitals auf die Lernergebnisse ergänzt (vgl. Anhang C), deutet darauf hin, dass ,die individuelle Teilnahme an lokalen Einrichtungen, die Zahl der spezifisch für das betreffende Viertel erstellten Programme wie auch das Ausmaß der verwandtschaftlichen und freundschaftlichen Beziehungen innerhalb des Viertels offenbar keinen großen Unterschied ausmachen". Die Autoren kommen vielmehr zu dem Schluss, dass „der Rückgang der Gewalt eher eine direkte Folge der informellen Kontrolle innerhalb der Gemeinschaft und des sozialen Zusammenhalts der Bewohner des betreffenden Viertels darstellt" (Sampson, Raudenbush und Earls, 1997, S. 918-924). 
Das Sozialkapital kann zu einer Verbesserung der öffentlichen Verwaltung führen ...

... und ist für die Verbesserung des Wohlergehens vielleicht noch wichtiger als das Humankapital.
Auch die Effizienz, mit der öffentliche Einrichtungen und staatliche Stellen die soziale Integration und den sozialen Zusammenhalt fördern, kann sehr stark vom Sozialkapital abhängen. Putnam (1993 und 2000b) zeigt mit Hilfe von Angaben zu Wählerverhalten, Steuerhinterziehung sowie bürgergesellschaftlichem und politischem Engagement, dass zwischen dem Sozialkapital und der Leistungsfähigkeit staatlicher Einrichtungen ein Zusammenhang besteht. Regionen bzw. Länder mit höherem Vertrauensniveau und stärkerem Engagement haben generell eine bessere Staatsverwaltung, auch unter Bereinigung um sonstige soziale und wirtschaftliche Faktoren. Außerdem ist bei Menschen, die an ihrem Wohnort, im Beruf oder in einem Verein über soziale Bindungen verfügen, die Wahrscheinlichkeit geringer, dass sie desinteressiert an lokalen politischen Aktivitäten sind und extreme Ansichten vertreten. Mit anderen Worten werden die sozialen und bürgergesellschaftlichen Kompetenzen in ehrenamtlichen Bürgervereinigungen, die Putnam als ,Schulen der Demokratie“ bezeichnet, gefördert. Die meisten Formen des bürgergesellschaftlichen Engagements, wenn auch nicht alle, tragen zu einem Klima des Vertrauens, der Gegenseitigkeit und der Zusammenarbeit bei, das positive Übergreifeffekte auf lokale Kommunen, gesellschaftliche Gruppen und Wirtschaftssysteme hat. In Bürgervereinigungen lernt der Einzelne, sich im Gespräch mit anderen, die oft gegenteilige Meinungen vertreten, auseinanderzusetzen.

Wie in Kapitel 2 dargelegt, befassen sich immer mehr Studien mit den Bestimmungsfaktoren der „Lebenszufriedenheit“ bzw. des subjektiven Wohlbefindens. Auf der Basis von Erhebungsergebnissen wird in diesen Studien der Zusammenhang zwischen dem Niveau des subjektiven Wohlbefindens oder Glücks auf der einen und demographischen, sozialen, ethnischen und sonstigen Faktoren auf der anderen Seite untersucht. Mangels hinreichender Daten, mit denen die Entwicklung des Einzelnen im Laufe der Zeit verfolgt werden kann, lassen sich nur schwer allgemeine Schlussfolgerungen ziehen. Jedoch sind der einschlägigen Fachliteratur eine Reihe sehr wichtiger Erkenntnisse zu entnehmen. Putnam (2000a) untersucht die Entwicklung des von den Befragten angegebenen subjektiven Glücksniveaus und der jeweils damit verbundenen persönlichen Merkmale (S. 333). Er kommt zu dem Ergebnis, dass neben der persönlichen Gesundheit die sozialen Bindungen offenbar der mit Abstand bedeutendste Bestimmungsfaktor sind. Auch der Familienstand hängt eng mit dem subjektiven Glücksgefühl zusammen. Putnam stellt fest, dass Humanund Sozialkapital das Glück sowohl auf individueller als auch auf allgemeinerer Ebene zu erhöhen scheinen ${ }^{25}$. Das regionale Durchschnittseinkommen beeinflusst das Wohlbefinden sehr viel weniger als Bildung, Gesundheit oder Sozialkapital. Unter Annahme sonst gleicher Bedingungen führt ein Anstieg des Durchschnittseinkommens auf der Ebene der US-Bundesstaaten nicht zu einer Steigerung des subjektiven Glücksempfindens, während eine Erhöhung des Human- oder Sozialkapitals auf Bundesstaatsebene sehr wohl diesen Effekt hat.

Von Blanchflower und Oswald (2000) untersuchte Belege für die Vereinigten Staaten und Großbritannien erhärten die These, dass soziale Bindungen für das persönliche Glück wichtiger sein können als Bildung und Einkommen zumindest in der Kategorie der durchschnittlichen bis überdurchschnittlichen Einkommen. Myers (1999) kommt zu ähnlichen Ergebnissen für die Vereinigten Staaten in Bezug auf die Auswirkungen enger persönlicher Beziehungen 
Sozialkapital und Gleichheit sind miteinander verbunden, wenn auch nicht feststeht, in welcher Richtung die Kausalbeziehung verläuft. und sozialer Bindungen auf die subjektive Lebensqualität. Blanchflower und Oswald untersuchen die Auswirkungen von Lebensereignissen, wie Heirat, Arbeitslosigkeit, Scheidung usw., auf das Glück. Wie auch Putnam (2000a) finden sie Belege dafür, dass das subjektive Glücksempfinden in der Zeit zwischen Anfang der siebziger und Ende der neunziger Jahre abgenommen hat. Obwohl zwischen Bildung und Glück eine wichtige Korrelation besteht, rangiert die Bildung dennoch nur auf Platz 3, nach den sozialen Bindungen und der Gesundheit. Auch das Einkommen spielt eine Rolle, jedoch nicht in demselben Maße wie soziale Bindungen, Gesundheit oder Bildung, und diese Rolle nimmt mit steigendem Einkommen ab. Diese Ergebnisse stehen mit den makroökonomischen Beziehungen zwischen Pro-Kopf-BIP und Gesamtniveau des Wohlergehens im Einklang, wie sie in einem Ländervergleich beobachtet wurden (Inglehart, 1997).

\subsection{Relation zwischen Sozialkapital und sozialer Ungleichheit}

Akute Formen der sozialen Ausgrenzung (auf Grund des sozialen, ethnischen, geschlechtlichen oder regionalen Status) gehen, insbesondere auf der Ebene der Brückenbeziehungen, mit geringerem Vertrauen und schwächerem bürgergesellschaftlichen Engagement einher. Gruppen sind möglicherweise weniger geneigt, mit anderen Gruppen zusammenzuarbeiten oder ihnen zu vertrauen, selbst wenn innerhalb der einzelnen Gruppen ein hohes Maß an Zusammenarbeit und Vertrauen herrscht. Nachstehend werden zwei miteinander zusammenhängende Fragen untersucht:

- Welche Wechselbeziehungen bestehen auf mikroökonomischer Ebene zwischen Sozialkapital und sozialer Ungleichheit (Zugang zu Bildung, Arbeitsplätzen und Einkommen)?

- Führt Sozialkapital auf makroökonomischer Ebene zu mehr Einkommens- und Chancengleichheit in der Gesellschaft, und sorgt diese Gleichheit wiederum für eine Festigung des Vertrauens und des sozialen Zusammenhalts?

Länder und Regionen, in denen Vertrauen und bürgergesellschaftliches Engagement ein hohes Niveau erreichen, weisen in Bezug auf das Einkommen, die Grundqualifikationen Erwachsener (literacy) sowie den Zugang zur Weiterbildung eine größere Chancengleichheit auf. Je höher der Grundstock an Sozialkapital ist, desto mehr zusätzliches Sozialkapital dürfte der Einzelne im Laufe seines Lebens erwerben. Die „Insider“ bestimmter Netzwerke und gesellschaftlicher Gruppen verfügen über umfangreiche Zugangsmöglichkeiten zu Ressourcen, von denen „Außenstehende“ ausgeschlossen sind. Hall (1999) berichtet beispielsweise über erhebliche Unterschiede des sozialen Engagements zwischen den verschiedenen gesellschaftlichen Gruppen im Vereinigten Königreich (ein Punkt, der bei Galland, 1999, auch in Bezug auf Frankreich entwickelt wurde). Hall kam zu dem Ergebnis, dass Personen aus dem Mittelstand tendenziell häufiger neuen Vereinen beitreten, im Laufe ihres Lebens mithin mehr Mitgliedschaften akkumulieren und sich diversen und weitreichenden sozialen Netzen anschließen. Hingegen treten Personen aus der Arbeiterklasse tendenziell weniger häufig Vereinen bei, wo sie oft ganz spezifische Aufgaben übernehmen und über einen langen Zeitraum Mitglied bleiben. 
Die wirtschaftlichen Ungleichheiten können gleichzeitig Ursache und Folge der Ungleichheiten auf dem Gebiet des Sozialkapitals sein.

Für die Verknüpfung von sozialer Ausgrenzung, Sozialkapital und Ungleichheiten im gesundheitlichen Bereich gibt es keine sicheren Belege.

Das Sozialkapital kann die soziale Spaltung entweder verfestigen oder aber überbrücken helfen.
Die in Abschnitt 3.6 erörterte Trendentwicklung des Sozialkapitals spiegelt möglicherweise Veränderungen in der Struktur der wirtschaftlichen Ungleichheiten in und zwischen den Ländern wider. Laut Putnam (2000a, S. 360) ist der Rückgang des Sozialkapitals in den Vereinigten Staaten durch die wachsenden Ungleichheiten bei Einkommen und Wohlstand bedingt. Der Kausalzusammenhang kann in beiden Richtungen wirksam sein; so liefert Putnam auch Belege dafür, dass sich Ungleichheit und geringes bürgergesellschaftliches Engagement in der Regel gegenseitig verstärken. Knack (1999) stellt ferner in verschiedenen Ländern eine positive Korrelation zwischen Einkommensgleichheit und Vertrauen fest. Hingegen vertritt Fukuyama (1999) eine ziemlich abweichende Auffassung, wenn er argumentiert, dass es in den Vereinigten Staaten der Zerfall der Familien und der daraus meist resultierende Vertrauensschwund sind, die bis zu einem gewissen Grad für die Zunahme der Armut verantwortlich sind.

Unter Verwendung von Daten, die auf der Ebene der Bundesstaaten im Rahmen der in den USA durchgeführten Allgemeinen Sozialerhebung (General Social Survey) erhoben wurden, argumentieren Kawachi et al. (1997), dass Einkommensungleichheiten das Sozialkapital verringern und auf diesem Wege zu höheren Sterblichkeitsraten (wie auch höheren Kriminalitätsraten) führen. Über die indirekte Rolle des Sozialkapitals hinaus gibt es zwischen Armut und schlechtem Gesundheitszustand natürlich auch direkte Verknüpfungen. Die Kernthese der Autoren lautet, dass die Einkommensverteilung auf der Ebene der einzelnen Bundesstaaten eine wichtigere Rolle für die Ergebnisse im Gesundheitsbereich spielt als das Durchschnittseinkommen ${ }^{26}$. Bestätigt wird diese Feststellung auch von Wilkinson (1996) und anderen, die berichten, dass gesundheitliche Ungleichheiten innerhalb der Länder oder Regionen in stärkerem Maße auf den Grad der Chancengleichheit als auf Unterschiede beim durchschnittlichen Einkommensniveau zurückzuführen sind. Halpern und Nazroo (2000) äußern ferner die Vermutung, dass Mitglieder ethnischer Minoritäten psychisch robuster sind und ihr persönliches Wohlbefinden besser ist, wenn sie in Gebieten leben, in denen ihre Gruppe besonders stark repräsentiert ist. Jedoch warnen Lynch et al. (2000) vor „einer zu stark vereinfachenden Interpretation der Relationen zwischen Sozialkapital, wirtschaftlicher Entwicklung, staatlicher Politik und Gesundheit“. Muntaner et al. (2000) vertreten ferner die Auffassung, dass die Nützlichkeit des Sozialkapitalkonzepts für den Gesundheitszustand der Bevölkerung insofern überbewertet wurde, als das Konzept zu eng definiert und seine Relevanz für die öffentliche Gesundheit zu optimistisch eingeschätzt wurde. Diesen kritischen Stimmen zufolge ist angesichts des schwachen konzeptuellen Fundaments und des Mangels an empirischen Belegen zum Thema Sozialkapital Vorsicht geboten.

In Ländern mit starker sozialer, ethnischer oder sprachlicher Polarisierung (Collier, 1998; Knack und Keefer, 1997) ist die Gefahr eines Auseinanderbrechens des Sozialgefüges zwangsläufig größer. Jedoch haben viele multiethnische Gesellschaften, in denen mehrere Sprachen gesprochen werden, unter Beweis gestellt, dass sie mit der Diversität umgehen und diese positiv nutzen können. La Porta et al. fanden beispielsweise keine signifikante Korrelation zwischen ethno-linguistischer Heterogenität und subjektiven Äußerungen des Misstrauens.

Wenn die Fragmentierung des sozialen Gefüges auch zur Bildung neuer Strukturen und Netzwerke führen kann, was eigentlich zu begrüßen ist, besteht doch die Gefahr einer ungleichen Verteilung der neuen Formen des Sozial- 
Das Sozialkapital kann Unterschiede bei den Grundkompetenzen allgemein bzw. bei den Lernergebnissen reduzieren helfen.

Vertrauen fördert die Produktivität ...

... wenn es in der organisatorischen Unternehmenskultur verankert ist. kapitals. Das Internet lässt Hoffnungen wie auch neue Herausforderungen entstehen, jedoch müssen diese Effekte abgewogen werden gegen die Risiken der sozialen Ungleichheit beim Zugang zum Cyberspace, gegen den als unpersönlich empfundenen Charakter des Internets, die weiter voranschreitende Aufspaltung der Gesellschaft sowie die Möglichkeit, dass sich dieses Medium zu einem privaten Instrument der passiven Unterhaltung entwickelt. Diese Gefahren dürften sich in Zukunft noch in dem Maße verstärken, wie bestimmte Gruppen wahrscheinlich Zugang zu neuen Technologien und Formen des Lernens haben werden, die ihre Chancen am Arbeitsmarkt verbessern.

Willms (2001) stellt unter Verwendung kanadischer Daten aus Schulleistungsstudien fest, dass bei Personen aus armen Verhältnissen, die außerdem in armen Volksgruppen leben, die Gefahr besonders groß ist, dass sie nur über einen niedrigen Bildungsabschluss verfügen. Volksgruppen mit einem hohen Niveau an Sozial- und Kulturkapital können höhere Lernerträge erzielen. Willms hebt die Bedeutung eines von Disziplin geprägten Klimas, des Engagements und der Anspruchshaltung der Eltern für die Anhebung des Niveaus der Grundqualifikationen und -kompetenzen hervor. Darüber hinaus spielen auch die Familie und die jeweilige gesellschaftliche Gruppe bei der Anhebung des Qualifikationsniveaus und der Verbesserung der Grundkompetenzen benachteiligter Erwachsener eine wichtige Rolle. Diese Ergebnisse legen ferner den Schluss nahe, dass die Maßnahmen, die eine Gesellschaft zur Überwindung des sozialen Handicaps ergreift, ein wichtiger Bestimmungsfaktor dafür sind, wie gut diese Gesellschaft bei den Grundkompetenzen (literacy) insgesamt abschneidet. Die zwischen den Ländern auf diesem Gebiet bestehenden Unterschiede sind bei den unteren sozioökonomischen Gruppen besonders groß, was darauf schließen lässt, dass die Ermittlung der Bedürfnisse der sozial Benachteiligten, und insbesondere der Menschen, die einen nur unbefriedigenden Zugang zu sozialen Netzen haben, ein Kernelement aller Strategien zur Verbesserung des globalen Niveaus der Grundkompetenzen darstellen muss.

\subsection{Auswirkungen des Sozialkapitals auf den wirtschaftlichen Wohlstand}

\subsubsection{Produktivität in Unternehmen und Organisationen}

Alle Transaktionen - seien sie privater, sozialer, wirtschaftlicher oder politischer Natur - basieren auf Vertrauen. Von zentraler Bedeutung für die Definition des Sozialkapitals ist das Konzept der Netzwerke ${ }^{27}$. Unternehmen können aus Normen der Zusammenarbeit und des Vertrauens, die in diversen betriebsinternen oder zwischenbetrieblichen Netzwerken verankert sind, insofern Nutzen ziehen, als diese die Koordination erleichtern und die Transaktionskosten reduzieren, die durch Verhandlungen und Bemühungen um Durchsetzung von Regeln, unzureichenden Informationsfluss sowie unnötige Bürokratie entstehen ${ }^{28}$. So gesehen ist Vertrauen ein mehrdimensionales Phänomen, zu dem auch der Glaube an die guten Absichten sowie an die Kompetenz und Verlässlichkeit der anderen Menschen gehört.

Humphrey und Schmitz (1998) heben hervor, dass ,in Deutschland, Japan und Teilen Italiens auf Vertrauen basierende Beziehungen zwischen den Wirtschaftsakteuren als einer der Gründe für den Wettbewerbsvorteil verarbeitender Unternehmen angesehen werden ....“. Hersteller und Käufer können dank wie- 
Vertrauen kann die Voraussetzung für größere und effizientere Produktionseinheiten sein ...

... wie auch für

eine verstärkte

innerbetriebliche

Zusammenarbeit.

Sozialkapital kann die Einrichtung regionaler Innovationssysteme erleichtern. derholter, auf Vertrauen und Vernetzung basierender Transaktionen langfristige Beziehungen der Zusammenarbeit und der wechselseitigen Verpflichtung aufbauen. Unternehmensnetze, die die Bereiche Marketing, Ausbildung bzw. Forschung abdecken, können langfristig Vorteile erzielen, indem sie ihre Gemeinkosten reduzieren, Informationen austauschen und opportunistisches Verhalten mit Sanktionen ahnden. In einigen Nischenmärkten, wie beispielsweise der Software-Entwicklung oder der Bekleidungsindustrie, sparen die Unternehmer dadurch Zeit, dass sie Informationen austauschen und sich rasch den wandelnden Bedürfnissen der Kunden anpassen (Uzzi, 1996 und 1997).

Einige Forscher haben die Konzepte des allgemeinen und des spezifischen Vertrauens vergleichend untersucht. La Porta et al. (1997) kamen zu dem Ergebnis, dass zwischen den in Prozent des BIP ausgedrückten Erträgen der 20 größten Unternehmen und dem allgemeinen Vertrauen in die Menschen ein positiver, zwischen dieser Variable und dem Vertrauen in die Familie hingegen ein negativer Zusammenhang besteht. Sie gehen von der These aus, dass größere Firmen eher in Gesellschaften vorherrschen, die sich durch ein relativ größeres Vertrauen auszeichnen und in denen Strafen und Sanktionen für opportunistisches Verhalten weniger notwendig sind. Ausgehend von der gleichen Hypothese würden hingegen Großunternehmen in einem eng gewebten sozialen Gefüge, das auf familiären und ethnischen Banden beruht, schwerer Fuß fassen. Bei diesem Modell ist das Vertrauen ein überwiegend exogener Faktor, der durch historische und kulturelle Faktoren geprägt wird und dazu dient, kollektive Aktionen und Zusammenarbeit zu erleichtern, einschließlich eines verstärkten bürgergesellschaftlichen Engagements und einer effizienteren öffentlichen Verwaltung.

Analog hierzu können betriebsinterne Netzwerke und Normen der $\mathrm{Zu}$ sammenarbeit die Teamarbeit erleichtern, Effizienz und Qualität steigern sowie den Informationsfluss und den Wissensstand verbessern. Shimada (1988) betrachtet den Geist der Zusammenarbeit zwischen Arbeitern und Management als Hauptgrund für die (traditionell) starke Wettbewerbsfähigkeit der japanischen Automobilhersteller: „In einem amerikanischen Unternehmen ist jeder Arbeiter darauf bedacht, persönliche Erfolge zu erzielen, jedoch nicht dazu bereit, sein Wissen mit seinen Kollegen zu teilen. Hier in Japan hingegen wird das Wissen bereitwillig so weit wie eben möglich unter den Kollegen weitergegeben, da jeder davon überzeugt ist, dass er nur im Team, nicht aber als Einzelner Erfolg haben kann“" (Omori, 2001). Die verschiedenen Formen des Sozial- und Organisationskapitals können je nach den Phasen der Wirtschaftsentwicklung mehr oder minder angemessen sein - wie der Rückgang der Wettbewerbsfähigkeit der japanischen Automobilhersteller in den neunziger Jahren gezeigt hat.

\subsubsection{Produktivität auf regionaler und lokaler Ebene}

Regionale Wirtschaftssysteme, die auf lokalen Lernnetzen fußen, sind potentiell flexibler und dynamischer als solche, in denen sich der Wissenserwerb auf die einzelnen Unternehmen begrenzt. Regionale bzw. lokale Lernnetze können den Informationsfluss und wechselseitige Lernprozesse begünstigen und darüber hinaus Skaleneffekte ermöglichen. Putnam (2000a) stellt den Erfolg des Silicon Valley und der Route 128 in den Vereinigten Staaten einander gegenüber. Im kalifornischen Silicon Valley trug eine Gruppe von Unter- 
ehmern mit Unterstützung von Forschern der dortigen Hochschulen zur Entwicklung eines Weltzentrums für fortgeschrittene Technologien bei. Putnam stellt fest, dass ,,der Erfolg weitgehend auf die informellen und formellen horizontalen Netze der Zusammenarbeit zurückzuführen war, die sich zwischen den neu etablierten Unternehmen in dieser Region bildeten“. Demgegenüber war es der Mangel an betriebsinternem Sozialkapital, der im Hightech-Korridor entlang der Route 128 in der Nähe von Boston zu einer traditionelleren Form der Unternehmenshierarchie führte, die durch Geschäftsgeheimnisse, Autonomie und das Territorialitätsprinzip gekennzeichnet ist.

\subsubsection{Arbeitsplatzsuche}

Der Zugang zu Sozialkapital erleichtert die Arbeitsplatzsuche.
Wie Barbieri, Russell und Paugam (1999) zeigen, stellt das Sozialkapital in mehreren europäischen Ländern eine nützliche Ressource für die Beschäftigungssuche dar, insbesondere an offenen und flexiblen Arbeitsmärkten. Wichtiger noch als die Zahl der sozialen Beziehungen, über die die betreffende Person verfügt, ist für eine erfolgreiche Arbeitsplatzsuche das Spektrum der Personen, mit denen diese in engerem Kontakt steht und auf die sie sich verlassen kann. Brückenbeziehungen sind für Arbeitsuchende wichtiger als auf Zuneigung beruhende Beziehungen. Jedoch haben Arbeitslose im Allgemeinen weniger Zugang $\mathrm{zu}$ weitreichenden Netzen und Kontakten innerhalb der Arbeitswelt.

Eine Reihe von Ökonomen, darunter auch Granovetter (1973), hat die Rolle sporadischer Kontakte wie auch enger Freunde und Familienangehöriger bei der Arbeitsplatzsuche hervorgehoben. Analog dazu unterstreicht Burt (1992), dass das Fehlen enger Beziehungen ${ }^{29}$ ein Faktor ist, der die individuelle Mobilität und den Wissensaustausch begünstigt. In geschlossenen oder dichten Netzwerken ist der Informationsfluss eher repetitiv. Laut Burt sind Informationen und Einfluss (auf die eigene Autonomie oder die anderer) zwei Arten von Vorteilen, die soziale Netze bieten. Eine dritte Art von Vorteilen kann in sozialer Solidarität gesehen werden, die Zusammenarbeit, den Sinn für soziale Verpflichtungen und Gegenseitigkeit voraussetzt, ohne Erwartung einer unmittelbaren Gegenleistung zu Gunsten der Personen, die zum Wohlergehen anderer beitragen. Loury (1987), einer der zahleichen Autoren, die unabhängig voneinander den Begriff des Sozialkapitals „erfunden“ haben, wollte damit veranschaulichen, dass weiße Amerikaner selbst bei Ausschaltung der Vorteile, über die sie in Bezug auf Human- und Finanzkapital verfügen, dank ihrer vielfältigeren Beziehungen zu großen amerikanischen Institutionen gegenüber den Mitgliedern der einer Minderheit angehörenden Mittelklasse begünstigt sind.

\subsubsection{Makroökonomische Vorteile}

Wie Arrow (1972) festgestellt hat, können zwischenmenschliches Vertrauen wie auch Normen des Vertrauens gegenüber den Institutionen für viele wirtschaftliche und soziale Aktivitäten von zentraler Bedeutung sein. Bisher ist die Rolle, die das Sozialkapital bei der Erklärung des unterschiedlichen Wirtschaftswachstums zwischen den Ländern spielt, aber nur in begrenztem Maße untersucht worden, da das Konzept noch recht neu und heterogen ist und sich geeignete, international vergleichbare Indikatoren nur schwer erstellen lassen. Ferner ergeben sich auf der Ebene der Aggregation große Messprobleme. 
Die jüngsten Daten betreffend den Einfluss des Vertrauens auf das Wirtschaftswachstum zeichnen ein uneinheitliches Bild ...

... und das Gleiche gilt für den Zusammenhang zwischen Vereinsmitgliedschaft und Wirtschaftswachstum.
Je nach den ausgewählten Ländern und dem untersuchten Zeitraum und je nachdem, ob andere Erklärungsvariablen in die Wachstumsgleichungen aufgenommen wurden, fallen die Ergebnisse in Bezug auf die makroökonomischen Effekte des Sozialkapitals unterschiedlich aus. Unter Verwendung von Daten der World Values Study stellten Knack und Keefer (1997) fest, dass - unter Bereinigung um das ursprüngliche Pro-Kopf-Einkommen, eine Humankapitalvariable sowie den relativen Preis von Investitionsgütern - eine positive Korrelation zwischen einer gegebenen Messgröße des allgemeinen zwischenmenschlichen Vertrauens und dem BIP-Wachstum besteht ${ }^{30}$. Unter Verwendung der gleichen Datenquelle fand Hjerrpe (1998) für eine Stichprobe von 27 Ländern (darunter auch eine Reihe von Ländern der mittleren und unteren Einkommensgruppe) ebenfalls einen positiven und signifikanten Zusammenhang zwischen Vertrauen und Wirtschaftswachstum ${ }^{31}$, nach Bereinigung um Faktoren wie Sachkapital, Marktöffnung für den Handel und Anteil der Erwachsenenbevölkerung mit Hochschulbildung. Helliwell (1996) hingegen stellte für eine Stichprobe von 17 OECD-Mitgliedsländern eine negative Korrelation zwischen Vertrauen und Wachstum der gesamten Faktorproduktivität fest.

Andere Forscher kamen zu dem Schluss, dass Vertrauen eine stimulierende Wirkung auf die Sparneigung, die Risikobereitschaft und die Investitionen haben kann. Guiso et al. (2000) berichten, dass in einigen Regionen Italiens, die sich durch ein hohes Niveau an sozialem Vertrauen auszeichnen, die Haushalte weniger in Bargeld als in Aktien investieren, mehr Schecks verwenden, leichter an offizielle Kredite herankommen und weniger auf informelle Kredite zurückgreifen. Auch die Unternehmen haben verstärkten Zugang zu Krediten und häufig einen größeren Aktionärsstamm. Das Vertrauen spielt offenbar besonders in solchen Regionen eine große Rolle, in denen die Durchsetzung des geltenden Rechts relativ gesehen schwächer ist, wie auch in weniger gebildeten Bevölkerungsgruppen. Das finanzielle Gebaren „Zugezogener“ wird weniger vom Niveau des Vertrauens an ihrem Herkunftsort als vielmehr durch das soziale Vertrauen in ihrem effektiven Lebensumfeld geprägt.

Auf subnationaler Ebene durchgeführte Analysen scheinen insofern aussagekräftiger zu sein, als sie implizit einigen eher verwirrenden kontextuellen und kulturellen Faktoren Rechnung tragen. Norditalien beispielsweise weist, unter Bereinigung um sonstige Faktoren, eine deutlich bessere Staatsverwaltung, leistungsfähigere Institutionen und eine effizientere Wirtschaftsentwicklung auf ${ }^{32}$. Putnam (1993) sowie Helliwell und Putnam (1999a) vertreten die Auffassung, dass es Norditalien dank eines solideren Sozialkapitals gelungen ist, die regionalen Reformen für ein im Vergleich zu Süditalien rascheres Wirtschaftswachstum zu nutzen. Zur Untermauerung ihrer These stellten sie Vergleiche zwischen Nord- und Süditalien an, bei denen sie einen Zusammenhang zwischen den jeweiligen regionalen Leistungen seit Mitte der achtziger Jahre und der nach den Reformen der siebziger Jahre größeren Zufriedenheit der Bürger mit der Regionalverwaltung im Norden des Landes nachweisen konnten $^{33}$. Bis Anfang der achtziger Jahre wies das reale Pro-Kopf-BIP in den verschiedenen italienischen Regionen einen Konvergenztrend auf. Seitdem haben sich die regionalen Disparitäten jedoch erneut vergrößert.

Auch die Beziehungen zwischen dem länderspezifischen Niveau der Gruppenzugehörigkeit und dem Wirtschaftswachstum sind unklar. Putnam (1993) kam zu dem Ergebnis, dass langfristig zwischen dem Niveau des bürgergesellschaftlichen Engagements und der Wirtschaftsentwicklung ein Zusammenhang besteht. Er begründet das damit, dass die Mitglieder bürgergesellschaftlicher 
Andere Forschungsarbeiten deuten darauf hin, dass die soziale Infrastruktur eine wichtige Rolle für das Wirtschaftswachstum spielt.

\author{
Wichtig ist, sich über \\ die Grenzen dieser \\ Forschungsarbeiten \\ im Klaren zu sein.
}

Organisationen im täglichen Umgang miteinander Vertrauen und soziale Normen sowie die Fähigkeit erlernen, effiziente öffentliche Aktionsnetze aufzubauen. Zivilorganisationen können ein dichtes horizontales Netzwerk und zahlreiche Möglichkeiten für den Erwerb des für das Sozialkapital wichtigen Vertrauens schaffen.

Andere Untersuchungen kommen $\mathrm{zu}$ weniger schlüssigen Ergebnissen, was darauf hindeutet, dass die Hilfsvariablen einige wichtige Dimensionen des Sozialkapitals möglicherweise gar nicht erfassen. Knack und Keefer (1997) kamen unter Verwendung von Indikatoren für die Häufigkeit der Gruppenmitgliedschaft aus der World Values Study zu dem Ergebnis, dass zwischen Vereinigungen, deren Funktion darin besteht, die Interessen ihrer Mitglieder zu verteidigen - wie Gewerkschaften, politische Parteien und Berufsverbände -, und dem Wirtschaftswachstum bzw. den Investitionsquoten nur ein geringer Zusammenhang besteht. Sie beobachteten ferner, dass zwischen anderen Vereinigungen wie religiösen oder kirchlichen, Bildungs-, Kunst-, Musik- oder kulturellen Organisationen einerseits und dem Wirtschaftswachstum andererseits kein, zwischen diesen Faktoren und den Investitionen hingegen ein signifikant negativer Zusammenhang besteht.

Es ist daher nicht klar, ob die Veränderungen in Bezug auf die Gruppenzugehörigkeit oder andere Formen sozialer Interaktion wirklich einen Einfluss auf die in jüngster Zeit beobachteten Unterschiede des Wirtschaftswachstums der einzelnen OECD-Länder gehabt haben. Beispielsweise geht der Rückgang der Gruppenzugehörigkeit und des bürgergesellschaftlichen Engagements in den Vereinigten Staaten mit einem raschen Anstieg des Pro-Kopf-Einkommens einher.

Zahlreiche Untersuchungen der jüngeren Zeit befassen sich mit den kurzfristigen Bestimmungsfaktoren des Wirtschaftswachstums. Wieder andere Forschungsarbeiten deuten darauf hin, dass die Länder in der Regel eine langfristig höhere Pro-Kopf-Produktion erreichen, wenn sie hohe Investitionsquoten in Sach- und Humankapital mit einer sehr guten „sozialen Infrastruktur“ vereinen $^{34}$ (vgl. Hall und Jones, 1999). Die Qualität der „,sozialen Infrastruktur“ wird in erster Linie durch die Effizienz der Institutionen und staatlichen Maßnahmen bestimmt, die das wirtschaftliche Umfeld prägen, innerhalb dessen Individuen und Unternehmen Investitionen tätigen, Ideen entwickeln und weitergeben sowie Waren und Dienstleistungen produzieren. Sie hängt eng mit den in Kapitel 1 beschriebenen politischen, institutionellen und rechtlichen (PIR-)Strukturen zusammen.

Bei der Interpretation der Rolle, die das Vertrauen bei internationalen Vergleichen des Wirtschaftswachstums spielt, ist Sorgfalt geboten. Das Vertrauen kann sehr wohl auch durch andere gesellschaftliche Aspekte bestimmt werden, die in den Regressionsanalysen des Wachstums nicht erscheinen, bzw. sie können mit derartigen Aspekten korrelieren. So können z.B. Korruption und eine mangelnde Durchsetzung des geltenden Rechts das Vertrauen schwächen und zugleich aus völlig anderen, hiervon unabhängigen Gründen die Wachstumsrate dämpfen. Wie Knack und Keefer (1997) feststellten, könnte Vertrauen in Ländern mit guten Wirtschaftsergebnissen sogar ein Produkt von Optimismus sein. Armut und wirtschaftliche Unterentwicklung können wiederum die Entstehung von Vertrauen behindern, da Menschen, die wirtschaftlich nicht abgesichert sind oder in einer wirtschaftlich unterentwickelten bzw. unsicheren Gesellschaft leben, weniger bereit sind, anderen zu vertrauen. 
Der Einfluss auf die makroökonomischen Ergebnisse könnte z.T. auch indirekt über höhere Investitionsquoten in Sach- und Humankapital sowie über bessere Leistungen der „sozialen Infrastruktur“ (bzw. PIR gemäß Kapitel 1) zustande kommen. La Porta et al. (1997) ${ }^{35}$ wie auch Knack und Keefer (1997) liefern Belege für Verknüpfungen zwischen Vertrauen und Leistungsindikatoren der Regierungsarbeit, wozu u.a. die Effizienz der Justiz und die Qualität der Beamten gehören. Jedoch geht aus diesen Studien hervor, dass zwischen Vertrauen und Wirtschaftswachstum (im Zeitraum 1970-1993) nur ein schwacher Zusammenhang bestand, während die Verknüpfung zwischen einer Vielzahl sonstiger Faktoren, wie Bildungsniveau und Sachkapitalinvestitionen, unter Berücksichtigung einer Reihe von Kontrollvariablen stärker war. Ferner weisen sie unter sonst gleichen Bedingungen auf eine positive Korrelation zwischen Bildungsniveau und Vertrauen hin ${ }^{36}$.

\subsection{Schlussbetrachtungen}

Abschließend ist festzustellen, dass das Sozialkapital offenbar wirtschaftliche und soziale Vorteile bietet ...

... und dass Humanund Sozialkapital sich gegenseitig verstärken dürften.

Selbst wenn die Forschungsergebnisse entsprechende Schlüsse nahe legen, gibt es bisher noch keine absolut verlässlichen Belege dafür, dass Sozialkapital und Wirtschaftswachstum generell miteinander verknüpft sind.

Der unzureichende Zugang zu Sozialkapital bildet sicherlich auch einen wichtigen Aspekt der sozialen Ausgrenzung.
Bei der Analyse von Studien, die auf aggregierten bzw. undifferenzierten Proxymessungen des Sozialkapitals fußen, ist Vorsicht geboten. Im vorliegenden Kapitel wurde jedoch auf der Basis eines breiten Spektrums empirischer Untersuchungen in mehreren Ländern gezeigt, dass das Sozialkapital wirtschaftliche, gesellschaftliche und persönliche Vorteile mit sich bringen dürfte.

Zwischen Sozial- und Humankapital besteht eine wechselseitige Beziehung. Selbst im Ländervergleich ist beispielsweise eine positive Korrelation zwischen bürgergesellschaftlichem Engagement und Vertrauen auf der einen und Bildungsniveau auf der anderen Seite festzustellen ${ }^{37}$.

Schwieriger ist es schon, einen eindeutigen Nachweis für die Verbindung von Sozialkapital und Wirtschaftswachstum zu erbringen. Wie im Fall des Humankapitals wird die Beweislage durch Probleme in Bezug auf Qualität und Erfassungsgrad der mit Hilfsvariablen durchgeführten Messungen, die Komplexität der zwischen den verschiedenen Bestimmungsfaktoren bestehenden Wechselbeziehungen wie auch durch die Schwierigkeit beeinträchtigt, dass Länder mit stark abweichenden kulturellen, institutionellen und historischen Traditionen miteinander verglichen werden. Wie von Temple (2001) angeregt, bietet sich hier ein lohnendes Feld für weitere Forschungsarbeiten.

Das im vorliegenden Kapitel untersuchte Datenmaterial in Bezug auf die Vorteile des Zugangs zu Sozialkapital ist hinreichend überzeugend, um die Behauptung aufstellen zu können, dass das Sozialkapital in alle Überlegungen über Maßnahmen zur Überwindung von Armut und sozialer Ausgrenzung mit einbezogen werden muss - in der Tat ist mit sozialer Ausgrenzung ja nichts anderes gemeint, als dass den betreffenden Personen der Zugang zu Sozialkapital verwehrt ist. 


\section{Anmerkungen}

1. De Tocqueville (1835) schrieb: „Die Amerikaner bekämpfen den Individualismus mit der Doktrin des wohlverstandenen Interesses“, und fügte hinzu, dass „,sie ohne jede Selbstkritik selbstgefällig zeigen wie ihre Selbstliebe sie ständig dazu bewegt, sich gegenseitig zu helfen und bereitwillig einen Teil ihrer Zeit und ihres Reichtums für das Wohlergehen des Staates zu opfern".

2. In den Augen Durkheims setzt sich die Gesellschaft aus „Organen“ (soziale Fakten) bzw. sozialen Strukturen zusammen, die in der Gesellschaft eine Vielzahl von Funktionen übernehmen.

3. Weber befasst sich insbesondere mit den Individuen sowie mit den Mustern und Regelmäßigkeiten menschlicher Handlungen. Sein Hauptinteresse gilt dabei solchen Handlungen, bei denen zwischen dem konkreten Anstoß und der letztendlichen Reaktion eindeutig Denkprozesse liegen (die den Handlungen ihren eigentlichen Sinn geben).

4. Jacobs (1961) definiert Sozialkapital als ein „Netzwerk von Nachbarschaftsbeziehungen“.

5. Die Weltbank bevorzugt folgende Definition des Sozialkapitals: „Es bezieht sich auf Institutionen, Beziehungen und Normen, die Qualität und Quantität der sozialen Interaktionen in einer Gesellschaft prägen. Immer mehr Belege zeigen, dass der soziale Zusammenhalt für das wirtschaftliche Wohlergehen und die nachhaltige Entwicklung der Gesellschaften von entscheidender Bedeutung ist. Das Sozialkapital ist nicht einfach die Summe der Institutionen, auf denen eine Gesellschaft fußt - es bildet vielmehr den Zement, der alles zusammenhält“" (http://www.worldbank.org/poverty/scapital/) whatsc.htm).

6. Manche Autoren möchten auch „Ansichten und Überzeugungen“ in die Definition des Sozialkapitals aufnehmen. Adler und Kwon (2000) definieren diese beispielsweise als gemeinsame strategische Visionen, Interpretationen und Bedeutungssysteme. „Ansichten und Überzeugungen“ hängen eng mit den Werten zusammen, auf die sich die Zusammenarbeit stützt.

7. Putnam (1993) führt als Beispiel die „Mitglieder der florentinischen Chorvereine an, die diesen Vereinen aus Freude am Singen und nicht etwa deshalb beitreten, weil ihre Teilnahme das toskanische Sozialgefüge stärkt".

8. Die angeführten Beispiele bedeuten nicht, dass alle oder doch die meisten Gruppen, deren Mitglieder eng zusammenhalten, negative Auswirkungen auf die Gesellschaft haben. Einige Formen der Zuneigung, wie beispielsweise in Familien oder ethnischen Gruppen, können mit positiven Gesundheits- und Beschäftigungseffekten verbunden sein, die wiederum auf andere Gruppen übergreifen.

9. Zu den Formen gesellschaftlicher Brückenbeziehungen mit Ausschließlichkeitscharakter können auch Formen extremer oder totalitärer Ideologien zählen.

10. Es wurden repräsentative nationale Erhebungen über grundlegende Wertvorstellungen und Überzeugungen der Öffentlichkeit in mehr als 65 Ländern durchgeführt. Diese bauen auf den Europäischen Wertestudien (European Values Studies) auf, die erstmals im Zeitraum 1981-1989 durchgeführt wurden. Eine zweite Serie internationaler Erhebungen erstreckte sich auf die Jahre 1990-1993, eine dritte und vierte Serie auf die Zeiträume 1995-1997 und 1999-2000. Wegen näherer Einzelheiten vgl. wvs.isr.umich.edu/index.html.

11. Das gemessene Vertrauensniveau scheint in den zwanzig Ländern, die in den Erhebungen von 1981 und 1990 berücksichtigt wurden, im Zeitverlauf recht konstant geblieben zu sein (bei einem Korrelationskoeffizienten von 0,91).

12. Diese Korrelation kann nicht auf einen möglichen Verzerrungseffekt zurückgeführt werden, der durch das höhere Pro-Kopf-Einkommen bedingt gewesen wäre: Unter Bereinigung um das Pro-Kopf-Einkommen war die Teilkorrelation zwischen Vertrauen und zurückgegebenen Portemonnaies sogar größer als die einfache Korrelation.

13. Bei der Analyse dieser Ergebnisse ist angesichts der Tatsache, dass bei Alleinerzieherhaushalten meist auch zahlreiche andere Benachteiligungsfaktoren gegeben sind, Vorsicht geboten. Joshi et al. (1999) kamen beispielsweise zu dem Ergebnis, dass Kinder alleinerziehender Mütter, die jeden Kontakt zum Vater des Kindes abgebrochen hatten, unter Bereinigung um sonstige Faktoren, wie laufendes Einkommen und Bildungsabschluss der Mutter, weder in Bezug auf ihre schulischen Leistungen noch auf ihr Verhalten schlechter abschnitten als andere Kinder.

14. Loury befasst sich hauptsächlich mit den Ungleichheiten des Sozialkapitals und dessen potentiellem Einfluss auf die Struktur der sozioökonomischen Klassen.

15. Diese Definition wird von der Weltbank verwendet: www.worldbank.org/poverty/scapital/sources/civil1.htm.

16. Hierauf wird in Kapitel 1 unter der Abkürzung PIR Bezug genommen.

17. Picciotto (1998) stellt fest: „Die Diskriminierung zwischen den Geschlechtern schadet dem Vertrauen und den Familienbeziehungen, beschränkt die sozialen Netze und mindert das Sozialkapital, das es einer Gesellschaft ermöglicht, auf gemeinsame Ziele hinzuarbeiten.“ 
18. De Hart und Dekker kamen ferner zu der Schlussfolgerung, dass dieser Zusammenhang unabhängig von Bildungsniveau, Geschlecht, Alter oder Einkommensniveau der befragten Person besteht.

19. Jedoch waren nicht erwerbstätige Frauen stärker engagiert als vollzeitbeschäftigte.

20. $\mathrm{Zu}$ den sonstigen Ländern, in denen ehrenamtliche Tätigkeiten eine große Rolle spielen, zählen Belgien, Bulgarien, Deutschland, Irland, die Slowakei und das Vereinigte Königreich.

21. Jedoch dürften nicht alle Formen und Ausprägungen von Sozialbeziehungen unbedingt gesundheitsförderlich sein. Manche sozialen Beziehungen können vielmehr als einengend empfunden werden bzw. ein der Gesundheit abträgliches Verhalten fördern.

22. Diese Ergebnisse bleiben auch unter Bereinigung um Faktoren wie Einkommen, Bildungsniveau, Rassen- und Familienstrukturen in den einzelnen Bundesstaaten gültig.

23. Der ICVS fasst die Ergebnisse von Erhebungen zusammen, bei denen die Opfer aller Formen von Kriminalität erfasst wurden. Diese Erhebungen fanden in den Jahren 1989, 1992 und 1996 statt. Wegen näherer Einzelheiten vgl. ruljis.leidenuniv.nl/group/ifcr/www/icvs/index.htm.

24. Ein erstaunliches Resultat dieser empirischen Untersuchung besteht darin, dass das soziale Vertrauen bei Bereinigung um Faktoren wie Ungleichheit und Eigennutz mit einem höheren Kriminalitätsniveau einhergeht. Darin könnte sich vielleicht die Tatsache widerspiegeln, dass bei einem bestimmten Niveau von Ungleichheit und Eigennutz ein gewisses Reservoir an „Opfern“ besteht. Das heißt, diese „Opfer“ bleiben trotz des wenig vertrauenswürdigen Verhaltens eines Teils ihrer Mitbürger vertrauensselig und lassen Wohnungs- und Autotüren offen.

25. Putnam (2000a) stellt in den Vereinigten Staaten auf lokaler, d.h. auf County-Ebene keine Auswirkungen des Sozialkapitals auf den Faktor Glück fest, auch wenn dieser Effekt auf privater Ebene fortbesteht. Zurückzuführen ist dies möglicherweise auf Messfehler auf Grund des kleineren Stichprobenumfangs.

26. Eine alternative Betrachtungsweise der Mittlerrolle des Sozialkapitals bei den Auswirkungen von Armut und Ungleichheit auf die Gesundheit findet sich bei Lynch et al. (2000). Diese Autoren vertreten die Auffassung, dass die Bedeutung des Sozialkapitals für die Gesundheit möglicherweise überzeichnet worden ist.

27. „Wo Menschen vertrauen und vertrauenswürdig sind und ständig mit anderen Bürgern in Kontakt stehen, verursachen unternehmerische und soziale Transaktionen geringere Kosten“ (Putnam, 2000a, S. 288).

28. Fukuyama (1999) definiert ein Netzwerk als „eine Gruppe individueller Akteure, denen informelle Normen bzw. Werte gemeinsam sind, die über die für gewöhnliche Markttransaktionen notwendigen Elemente hinausgehen“.

29. Von Burt als ,structural holes“ (Strukturlöcher) bezeichnet.

30. Knack und Keefer kommen zu dem Ergebnis, dass eine Veränderung des Vertrauensindex um eine Standardabweichung mit einer Veränderung der Wachstumsrate um 0,56 einer Standardabweichung einhergeht. Mit anderen Worten bedeutet ein um 10 Prozentpunkte (etwas weniger als eine Standardabweichung) höheres Vertrauensniveau eine um 0,8 Prozentpunkte höhere jährliche Wachstumsrate.

31. Von den Nicht-OECD-Ländern wurden Argentinien, Brasilien, Chile, China und Russland einbezogen.

32. Putnam verwendete aggregierte Zeitreihendaten für 20 Regionen Italiens, die sich auf den Zeitraum 1960 bis Mitte der achtziger Jahre bezogen.

33. Bei der Messung des Sozialkapitals berücksichtigten sie namentlich folgende Elemente: Anteil der Bürger, die Zeitung lesen, Zahl der Sport- und Kulturvereine, Wahlbeteiligung bei Volksbefragungen, Bedeutung von Präferenzwahlsystemen sowie Effizienz der jeweiligen Regionalverwaltungen.

34. Die soziale Infrastruktur wird für zahlreiche Entwicklungsländer an folgenden Kriterien gemessen: $a$ ) Einrichtungen, die der Produktion mehr Bedeutung beimessen als dem Transfer, $b$ ) Offenheit für den internationalen Handel, $c$ ) Gewährleistung von Rechtsstaatlichkeit und Eigentumsrechten, $d$ ) Präsenz einer internationalen Sprache, sowie $e$ ) Entfernung vom Äquator.

35. Die Autoren kommen zu dem Ergebnis, dass ein Anstieg des in allen Ländern auf der gleichen Basis ermittelten Vertrauensindex um eine Standardabweichung die Effizienz der Justiz um 0.7 einer Standardabweichung erhöht und die Korruption im öffentlichen Sektor um 0,3 einer Standardabweichung reduziert.

36. Knack und Keefer beobachten für 1980 eine starke Korrelation $(r=0.83)$ zwischen Vertrauen und geschätzter durchschnittlicher Schulbesuchsdauer (in Jahren) und stellen fest, dass „Bildung Vertrauen und bürgerliche Normen stärken kann, wenn beispielsweise Ignoranz zu Misstrauen führt oder wenn Bildung die Ungewissheit in Bezug auf das Verhalten anderer reduziert oder wenn Schüler dazu angehalten werden, sich kooperativ zu verhalten" (S. 1270).

37. Beispielsweise geht mit einem Anstieg der Bildungsnachfrage auf Grund des Wandels der Arbeitsmarktbedingungen nicht unbedingt eine Erhöhung des Sozialkapitals einher. 
Kapitel 4

\section{KONSEQUENZEN FÜR DIE STAATLICHE POLITIK UND WEITERER FORSCHUNGSBEDARF}

„Welche Schwierigkeiten auch immer bei der Gestaltung und Evaluierung der Bildung auftreten mögen, es steht hierfür ein genuin politisches Instrument bzw. eine Vielzahl davon unmittelbar zur Verfügung. Es gibt Bildungsminister, und sie haben Aufgaben und Haushaltsmittel. Doch stellen wir uns einmal vor, wir seien der Minister für Sozialkapital: An wen würden wir uns wenden, wenn wir morgens zum Dienst kämen? Sozialkapital ist an sich ein ressortübergreifendes Konzept. "Helliwell (2001).

\author{
Human- und \\ Sozialkapital leisten \\ einen Beitrag zum \\ Wirtschaftswachstum \\ und zum Wohlergehen \\ der Bevölkerung.
}

\subsection{Einleitung}

Aus dem vorliegenden Bericht geht hervor, dass die politischen Entscheidungsträger dem Human- und Sozialkapital im Wesentlichen aus vier Gründen Interesse entgegenbringen sollten:

1. Es gibt solide Befunde, die zeigen, dass Humankapital ein wichtiger Bestimmungsfaktor des Wirtschaftswachstums ist, und auch wenn der vom Sozialkapital ausgehende Wachstumseffekt noch nicht nachgewiesen werden konnte, liegen doch genügend Daten vor, die eine weitere Untersuchung dieser Möglichkeit rechtfertigen.

2. Es hat sich gezeigt, dass Humankapital und Sozialkapital mit einer ganzen Reihe nicht ökonomischer Nutzeffekte in Zusammenhang stehen, z.B. Verbesserungen im Bereich der Gesundheit und des subjektiven Wohlergehens. Besonders wichtig sind solche Erkenntnisse, wenn sie mit Daten in Relation gesetzt werden, die zeigen, dass sich das Wohlergehen im Durchschnitt laut eigenen Angaben der Befragten in den letzten Jahren im OECD-Raum nicht im Gleichschritt mit dem BIP erhöht hat.

3. Sozialkapital und Humankapital können sich gegenseitig verstärken.

4. Den vorstehenden Aussagen zufolge kommt dem Human- und Sozialkapital, was die Lebensperspektiven des Einzelnen betrifft, eine so hohe Bedeutung zu, dass diesen beiden Arten von Kapital bei Maßnahmen zur Förderung der sozialen Integration Rechnung zu tragen ist.

Human- und Sozialkapital ermöglichen es dem Einzelnen, Gruppen, Unternehmen und der Gesellschaft, sich den Erfordernissen raschen sozialen und wirtschaftlichen Wandels anzupassen - d.h. Kindern beizubringen, mit einer sich wandelnden Welt umzugehen, Erwachsene für neue Beschäftigungsarten umzuschulen sowie das Vertrauen zu vermitteln und den Gemeinsinn zu wecken, von dem die meisten sozialen und wirtschaftlichen Aktivitäten abhängig sind. Bei dieser Art von Kapital handelt es sich um eine Ressource, die für die nachhaltige Entwicklung von entscheidender Bedeutung ist. 
Die Stärkung des Human- und Sozialkapitals ist die Aufgabe aller - und mit Sicherheit nicht nur die der Regierungen.

\section{Bei der Gestaltung von Maßnahmen zur Förderung des Human- kapitals muss der jeweilige Kontext berïcksichtigt werden.}

Bildungs- und Ausbildungseinrichtungen sind zwar für die Entwicklung des Humankapitals von großer Bedeutung, doch kommt es für den Erfolg dieser Einrichtungen entscheidend darauf an, dass sie auf breiter Ebene von der Gesellschaft unterstützt werden. Human- und Sozialkapital entstehen - formell und informell - am Arbeitsplatz, in gesellschaftlichen Gruppen auf lokaler Ebene und in der Familie. Sozialkapital ist Bestandteil freiwillig eingegangener sozialer Beziehungen, was bedeutet, dass die Regierungen meist eher die Entwicklung des Sozialkapitals erleichtern oder fördern und nicht so sehr selbst aktiv für seine Entstehung verantwortlich sind. Hinzu kommt, dass sich Sozialund Humankapital gegenseitig verstärken. Aus all diesen Gründen richten sich die hier dargelegten allgemeinen Überlegungen nicht nur an die Regierungen, sondern zugleich an eine ganze Reihe anderer Akteure und Einrichtungen - wie z.B. die Arbeitgeber und die in Freiwilligenorganisationen sowie in den wichtigsten Bereichen des öffentlichen Sektors tätigen Personen. Allen kommt eine Rolle zu.

\subsection{Maßnahmen zur Förderung des Humankapitals}

Bei der Konzipierung von Maßnahmen zur Förderung des Humankapitals ist folgenden Parametern Rechnung zu tragen:

- Wirtschaftliche Veränderungen - vor allem der höhere Grad der Wissensintensität der Wirtschaftstätigkeit - können die Nachfrage nach Humankapital vor allem insofern verändern, als sich das Schwergewicht stärker auf eine Reihe nicht kognitiver Qualifikationen wie z.B. Fähigkeit zur Teamarbeit und Innovation verlagert ${ }^{1}$.

- Jedes Lernumfeld - darunter auch Familie, Arbeitsplatz und der Vorschulbereich - ist wichtig.

- Partnerschaft und Dialog - zwischen dem privaten, öffentlichen und freiwilligen Sektor - sind von grundlegender Bedeutung, ebenso die Koordination der Maßnahmen - denn so entsteht eine Querverbindung zwischen der Bildung und Maßnahmen im Bereich der Beschäftigung und sozialen Sicherung.

- Sowohl im Hinblick auf den Zugang zu Aus- und Weiterbildung als auch bezüglich der Wahrnehmung des entsprechenden Angebots gibt es wesentliche Unterschiede, und diese müssen bei der Konzipierung von Maßnahmen für das lebensbegleitende Lernen berücksichtigt werden, wenn das Problem der sozialen Ausgrenzung erfolgreich angegangen werden soll.

- Sowohl bei der Erschließung als auch bei der Erosion des Human- und Sozialkapitals handelt es sich meist um Prozesse, die sich über längere Zeiträume erstrecken.

Die hier untersuchten Arbeiten über Humankapital haben, wenn sie in den Zusammenhang mit anderen Studien und Arbeiten über die Strategieentwicklung eingebettet werden, z.T. unmittelbare Konsequenzen für die staatliche Politik, und einige der vorgeschlagenen Optionen verdienen es, eingehender geprüft zu werden: 
Der vorliegende Bericht enthält eine Reihe von Optionen für die Humankapitalentwicklung.
1. Durch Humankapitalinvestitionen entstehen bedeutende private und soziale Nutzeffekte. Die jüngsten Forschungsarbeiten bestätigen die Auffassung, dass Humankapital eine wichtige Rolle für das Wirtschaftswachstum spielt. Überdies gibt es zahlreiche Belege dafür, dass durch Humankapitalinvestitionen beträchtliche nicht ökonomische Nutzeffekte entstehen - Verbesserungen im Bereich der Gesundheit und des Wohlergehens der Bevölkerung und der Kindererziehung durch die Eltern sowie ein stärkeres soziales und politisches Engagement. Veränderungen der Wirtschaftsstruktur erhöhen zudem insofern die Nachfrage nach Qualifikationen, als die Wirtschaftstätigkeit wissensintensiver wird und das Humankapital für die Wettbewerbsfähigkeit der Unternehmen und Staaten eine immer zentralere Rolle spielt. Dies bedeutet zwar nicht, dass jede Art von Bildungsinvestition positiv zu werten ist, doch wird so die Auffassung wesentlich erhärtet, dass gut fokussierte Investitionen zu Gunsten der Humankapitalbildung eine sinnvolle staatliche Maßnahme sind.

2. Das Sozialkapital hängt mit dem Bildungsabschluss zusammen, was darauf hindeutet, dass verschiedene von der Bürgerschaft getragene Netze die meisten Arten von Bildungs- und Ausbildungsaktivitäten positiv beeinflussen könnten. Durch Partnerschaften muss eine Plattform geschaffen werden, die auf das Lernen vorbereitet und beim Lernen unterstützt, und dabei ist der Tatsache Rechnung zu tragen, dass eine derartige Plattform oft zu einem großen Teil durch das häusliche und familiäre Umfeld gebildet wird² .

3. Es müssen Anreize für ständiges Lernen geschaffen werden. Einiges deutet darauf hin, dass eine Neuorganisation des Lernangebots über den ganzen Lebenszyklus sowie finanzielle und steuerliche Anreize zum Lernen ins Auge gefasst werden sollten, um den Erfordernissen des einzelnen Lernenden besser gerecht zu werden. Es besteht die Notwendigkeit, innovative Formen der Kofinanzierung wie z.B. individuelle Lernkonten zu fördern und rigoros zu evaluieren. So könnten die Schaffung von Anreizstrukturen für Aus- und Fortbildung am Arbeitsplatz, Teilzeitweiterbildung, Wechsel zwischen Arbeits- und Bildungszeiten sowie Rückgriff auf Fernstudien und IT-basiertes Lernen für Erwachsene erwogen werden.

4. Lehrpläne und Didaktik müssen neben kognitiven Fähigkeiten auch interpersonalen und anderen nicht kognitiven Fähigkeiten Gewicht beimessen. Effiziente Organisationen sind immer häufiger lernende und innovierende Organisationen. Auf Grund dieser Tendenz bekommen Eigenschaften wie Teamfähigkeit, Flexibilität und Innovationsvermögen sowie die Fähigkeit, mit Wissen umzugehen, einen immer höheren Stellenwert (vgl. OECD, 2001, Kap. 4).

5. Humankapitalinvestitionen können auch zur Entwicklung von Sozialkapital beitragen. Eine Schwerpunktverlagerung hin zu gruppenorientierten Problemlösungsansätzen könnte mit dafür sorgen, Schüler/ Studenten auf kooperativere Verhaltensweisen vorzubereiten. Die Bildungseinrichtungen könnten auch eingesetzt werden, um die Stärken lokaler gesellschaftlicher Gruppen weiter auszubauen, indem die Tatsache genutzt wird, dass weite Teile der jeweiligen Gruppen bereits Beziehungen zu derartigen Einrichtungen unterhalten. Gemeinschafts- 
aktivitäten könnten auf Zeiten oder Tage gelegt werden, an denen schulische Räumlichkeiten nicht für den normalen Schulbetrieb genutzt werden. (Szenario 3, Kap. 5, Education Policy Analysis, OECD, 2001, beschreibt die Rolle, die den Schulen in diesem Zusammenhang zukommen könnte.)

6. Aus- und Fortbildungsprogramme müssen auf den Personenkreis zugeschnitten sein, der am ehesten von der Ausgrenzung am Arbeitsmarkt bedroht ist. Im Allgemeinen ist die Wahrscheinlichkeit der Teilnahme an Ausbildungsmaßnahmen bei denen am geringsten, deren Bedarf am größten ist. Die in den letzten Jahrzehnten in vielen OECDLändern zu beobachtende Zunahme der Einkommensunterschiede könnte sich mit der Entwicklung neuer Lernformen und Technologien noch verschärfen. Um diese Probleme zu überwinden - und z.B. dafür zu sorgen, dass die digitale Kluft keine inakzeptablen Ungleichheiten beim Zugang zu Fernstudiengängen entstehen lässt -, wird es neuer Arten von Maßnahmen bedürfen.

\subsection{Maßnahmen zur Förderung des Sozialkapitals}

Zugang zu Sozialkapital ist generell vorteilhaft ...

... doch ist die Rolle des Staats bei der Stärkung des Sozialkapitals weniger eindeutig als im Zusammenhang mit dem Humankapital ...
Die hier untersuchten Befunde zeigen, dass das Sozialkapital in Bezug auf Wohlergehen, Gesundheit und Arbeitsuche eine wichtige Rolle spielt, und lassen gewissermaßen ahnen, welche Bedeutung ihm potentiell im Hinblick auf die Stützung des Wirtschaftswachstums zukommen kann. Dessen ungeachtet sind bestimmte Arten des Sozialkapitals nicht positiv zu werten - vor allem in Fällen, in denen Menschen zu unsozialen Zwecken kooperieren. Bei der Gestaltung einschlägiger Maßnahmen müssen die verschiedenen Arten von Sozialkapital und die Frage berücksichtigt werden, in welchem Grad diese erwünscht sind.

Wie weiter oben ausgeführt, ist die Rolle, die dem Staat bei der Stärkung des Sozialkapitals zukommt, nicht so klar wie im Fall des Humankapitals. Der Staat dürfte in der Regel eher einen fördernden Beitrag leisten, als die Rolle des Hauptakteurs spielen. Andererseits haben die Regierungsbehörden zusammen mit anderen öffentlichen Stellen einen zwar diffusen, aber insgesamt doch bedeutenden Einfluss auf die Entstehung von Sozialkapital. Instanzen, deren Maßnahmen Einfluss auf das Sozialkapital haben, finden sich über den ganzen öffentlichen, ehrenamtlichen und privaten Sektor verteilt. Den nachgeordneten lokalen und regionalen Verwaltungsebenen kommt meist eine besonders wichtige Rolle zu.

Am wichtigsten ist es, bei der Konzipierung von Maßnahmen zur Bekämpfung sozialer Ausgrenzung der Tatsache Rechnung zu tragen, dass der Zugang zu Sozialkapital ein entscheidender Faktor im Hinblick auf die Lebensperspektiven darstellt. Spezifische Arten des Sozialkapitals können zudem in Bezug auf die soziale Ausgrenzung besonders relevant sein - vor allem Sozialkapital, das einen „Brückenschlag“, d.h. die Aufnahme sozialer Beziehungen, ermöglicht, die über die Grenzen sozialer Schichten, ethnischer Gruppen oder des Geschlechts hinausgehen. Die Nutzung derartiger Brücken, um sich sozialen Netzwerken anzuschließen, denen in Bezug auf Zugang und Einfluss eine Schlüsselstellung zukommt, kann für den Erfolg von Migranten und Angehörigen ethnischer Minderheiten eine entscheidende Rolle spielen. Eine Handlungs- 


\section{Kasten 4.1 Maßnahmen zur Förderung des Sozialkapitals: Beispiele}

Maßnahmen zur Förderung des Sozialkapitals sind an sich nicht neu. Im Folgenden werden einige Beispiele für Maßnahmen angeführt, deren Effekte vom Sozialkapital abhängen bzw. die darauf hinwirken, das Sozialkapital zu verbessern:

Stärkung einzelner gesellschaftlicher Gruppen und von Familien zu Gunsten von Kindern (Italien). In Pistoia (Italien) stellt die Stadtverwaltung Räumlichkeiten für Kinder bereit und bietet so Hilfe für Familien an, die nicht auf eine Ganztagsbetreuung angewiesen sind. Eltern, Großeltern und andere Familienmitglieder können dort an Bereicherungsaktivitäten teilnehmen, und diese Räume dienen zugleich für Zusammenkünfte von Erwachsenen und als Spielund Erfahrungsbereich für Kinder. Sie werden auch für schulpflichtige Kinder genutzt, die sich dort nach dem Unterricht aufhalten können, sowie als Bildungsressourcenzentren für Lehrkräfte der städtischen Scuole dell'infanzia und der Grundschulen.

Brücken schlagen durch Einbeziehung gesellschaftlicher Gruppen (Nordirland). Das Ulster People's College, eine gemeinnützige Organisation in Belfast, bietet für die einheimischen Teilnehmer an freiwilligen und gemeinnützigen Aktivitäten strukturierte Ausbildungsprogramme verschiedener Niveaus an. Die Programme „Leitung von Gemeindeaktivitäten“ des College helfen aktiven Mitgliedern gesellschaftlicher Gruppen, ihre Arbeit effizienter zu gestalten, und das College ist zugleich ein gut funktionierendes Netzwerk für Personen, die in Belfast gemeinnützige und Gemeindearbeit leisten. Es gehört explizit zu den Aufgaben des College, Vertreter der Nationalisten und Unionisten einzubeziehen und einen Beitrag zum Friedens- und Versöhnungsprozess in Nordirland zu leisten.

Innovation durch Vernetzung (Dänemark). In Dänemark gibt es eine Reihe von Programmen, deren Ziel darin besteht, den Innovationsprozess durch Querverbindungen zwischen etablierten Forschungszentren und dem privaten Sektor zu fördern. Öffentlich/private Partnerschaften im Bereich der Forschung werden durch neue Gesetze über Erfindungen und neue Regelungen für die finanzielle Beteiligung des privaten Sektors an öffentlichen Forschungseinrichtungen gefördert. Innovation, Partnerschaft und Vernetzung werden auch durch „Zentrumsverträge“ gefördert, in deren Rahmen die Wirtschaft, technologische Dienstleistungseinrichtungen und Wissenschaftler an kommerziell ausgerichteten FuE-Projekten mitarbeiten und die im Wege der Mischfinanzierung vom Staat und von der Privatwirtschaft getragen werden.

\section{... während die}

Forschung im Bereich des Sozialkapitals noch in den Kinderschuhen steckt. option könnte darin bestehen zu versuchen, das Sozialkapital in Bereichen oder Gemeinschaften zu verbessern, in denen nachweislich ein Defizit an Sozialkapital besteht.

Einige Beispiele für bereits existierende Maßnahmen, die sich auf das Sozialkapital auswirken, sind in Kasten 4.1 aufgeführt. Bei neueren Untersuchungen über Sozialkapital wird die Bedeutung solcher Initiativen insofern akzentuiert, als sie den Nachweis erbringen, dass in einem bestimmten Kontext erworbene soziale Kontakte einer ganzen Reihe anderer Zwecke dienen können. Das bedeutet zum Beispiel, dass die Teilnahme an Sportaktivitäten weiterreichende Nutzeffekte haben kann, die über körperliche Gesundheit und den Spaß an sportlicher Betätigung hinausgehen.

Die Forschung im Bereich des Sozialkapitals befindet sich derzeit noch im Frühstadium, so dass bislang noch keine verlässliche Aussage darüber möglich ist, ob es mit bestimmten Programmen oder Maßnahmen gelingen wird, die vorgegebenen Ziele im Hinblick auf das Sozialkapital zu erreichen. Zugleich ist es nur durch die Konzipierung von Maßnahmen und deren Evaluierung möglich, zu einem besseren Verständnis des Sozialkapitals und seiner Nutzeffekte zu gelangen. Bei den nachfolgend dargelegten Optionen handelt es sich daher eher um Ideen, die es verdienen, weiterentwickelt, im Rahmen von Pilotprojekten erprobt und evaluiert zu werden, als um Programme, die bereits auf umfangreichen Forschungsarbeiten aufbauen können. 


\author{
Einige Ideen könnten \\ im Rahmen von Pilot- \\ vorhaben umgesetzt \\ und evaluiert werden.
}

1. Maßnahmen zur Unterstützung von Familien. Mögliche Politikoptionen betreffen z.B. steuerliche Hilfen, flexible Arbeitszeiten und Regelungen zur Förderung bzw. Erleichterung einer intensiveren Teilnahme der Eltern am Leben ihrer Kinder. Eine Flexibilisierung der Arbeitszeit im Sinne zusätzlicher Freizeit für Eltern sowie einer besseren Planung und Einteilung der Arbeitszeiten könnte dies erleichtern - wobei der Tatsache Rechnung zu tragen ist, dass familienfreundliche Maßnahmen dieser Art einen doppelten Effekt haben, denn sie bringen nicht nur eine Erleichterung für berufstätige Eltern, sondern veranlassen auch mehr Eltern, und namentlich Frauen, zur Aufnahme einer Erwerbstätigkeit, falls sie diesen Wunsch haben.

2. Maßnahmen zur Unterstützung freiwilliger Initiativen. Zu den Politikoptionen gehören sowohl nachfrageseitige Fördermaßnahmen zu Gunsten der Finanzierung von Organisationen, die effizient auf ehrenamtliche Mitarbeiter zurückgreifen, als auch angebotsseitige Maßnahmen, die die Arbeitgeber dazu bewegen, Beschäftigte für bestimmte Arten gemeinnütziger Aktivitäten freizustellen.

3. Entscheidungsprozesse in der öffentlichen Verwaltung. Demokratische Prozesse, die auf Bürgerbeteiligung setzen, sind nicht nur ein Mittel für eine bessere Entscheidungsfindung, sondern zugleich ein wesentliches Element im gesamten sozialen Gefüge. Mündige Bürger und ein bürgernaher Staat können dazu beitragen, das Gemeinwesen auf lokaler Ebene zu stärken und in der Gesellschaft wertvolle Energien für positive Aktionen freizusetzen.

4. IKT und Sozialkapital. Neue IK-Technologien können zusammen mit traditionellen Medien dazu genutzt werden, den Menschen zu helfen, Verbindung mit ihrem unmittelbaren lokalen Umfeld sowie mit weiter entfernten Gemeinschaften aufzunehmen. IKT bieten dem Staat neue Möglichkeiten, den Bürger zu konsultieren und mit ihm zu kommunizieren und staatliche Aktionen der Prüfung durch die Öffentlichkeit zu unterziehen. Elektronische Netzwerke können auch dazu dienen, Informationen zu übermitteln und vor allem dort, wo es an ergänzenden Informationen fehlt, Markttransaktionen $\mathrm{zu}$ erleichtern ${ }^{3}$. Die neuen Medien erweitern die Möglichkeiten informellen Lernens, z.B. durch Fernstudien. Es kommt entscheidend darauf an, den Zugang benachteiligter Gruppen zu den neuen Medien zu erleichtern, damit sie die Vorteile der neuen Informationsautobahnen und -netzwerke besser nutzen können. Mit zunehmender Hinwendung der Länder zu vielfältigen, nachfragebasierten und individuellen Arten des Wissenserwerbs wird informellen Kontexten eine immer wichtigere Rolle zukommen.

5. Anbindung der Gesundheitsversorgung an die Gemeinden. Die Planung der Gesundheitsversorgung bzw. der Gesundheitsdienste auf der Ebene der Gemeinschaften vor Ort, die es älteren Menschen und anderen Gruppen ermöglicht, den näheren Umkreis ihrer Familie und ihre gewohnte Umgebung nicht verlassen zu müssen, könnte ein weiteres Mittel sein, diese sozialen Bindungen zu erhalten und zugleich positive Effekte auf den Gesundheitszustand zu erzielen, die den Untersuchungen zufolge mit dem Sozialkapital in engem Zusammenhang stehen ${ }^{4}$. 
Weitere Arbeiten

über die Messung von

Humankapital, über

Nachfrageverände-

rungen sowie die Ver-

bindungen zwischen

Human- und Sozial-

kapital wären sinnvoll.
Durch weitere

Forschungsarbeiten

könnten das Sozial-

kapitalkonzept geklärt

und bessere Mess-

verfahren entwickelt

werden.

\subsection{Wissensdefizite und künftige Forschungsbereiche}

Im vorliegenden Bericht wurden zahlreiche Themen angesprochen und eine Vielzahl von Ansätzen für die künftige Forschung aufgezeigt. Eine Zusammenstellung von Vorschlägen ist zwangsläufig in hohem Maße selektiv. Die größten Wissens- und Verständnislücken bestehen offenbar in folgenden Bereichen:

1. Erweiterung der Messung von Humankapital - insbesondere der direkten Messung der Fähigkeiten, die über den Bereich der Grundkompetenzen in Lesen, Schreiben und Rechnen hinausgehen - um Bereiche wie z.B. die Fähigkeit, im Team zu arbeiten, Probleme zu lösen und mit IKT umzugehen. Selbst wenn solche Fähigkeiten bzw. gewisse Dimensionen dieser Fähigkeiten vom kulturellen Umfeld abhängen dürften, ist es dennoch wichtig - auch in Fällen, in denen z.B. das in internationalen Erhebungen verwendete Instrumentarium ungeeignet ist -, die Messverfahren zu verbessern. Im Rahmen der derzeitigen Aktivitäten in dieser Hinsicht werden u.a. die Anstrengungen fortgesetzt, um auf der Basis der Internationalen Erhebung über Grad und Verteilung elementarer Grundqualifikationen Erwachsener (IALS) eine breitere Palette von allgemeinen sozialen Kompetenzen Erwachsener aufzubauen.

2. Herstellung eines besseren Verständnisses der Art und Weise, wie sich die Humankapitalnachfrage gegenwärtig und künftig in quantitativer wie in qualitativer Hinsicht verändert. Dieser Frage wird eine entscheidende Bedeutung bei der Ausrichtung der gegenwärtigen Initiativen zukommen, die darauf abzielen, einerseits Wissensarbeiter für die New Economy heranzubilden und andererseits die Probleme anzugehen, mit denen diejenigen konfrontiert sind, die über zu geringe oder nicht mehr zeitgemäße Grundqualifikationen verfügen. Weitere Arbeiten sind möglicherweise erforderlich, um in die Überlegungen über die sich wandelnde Qualifikationsnachfrage einerseits die Struktur der Schulcurricula und andererseits die nötige Ausgewogenheit zwischen der schulischen Grundausbildung und dem lebensbegleitenden Lernen einzubeziehen.

3. Klärung der Beziehungen zwischen Humankapital und Sozialkapital, um herauszufinden, wie soziale Netzwerke die Bildung des Einzelnen fördern können und wie Bildung das Sozialkapital erhöhen kann.

4. Konzeptuelle Weiterentwicklung des Sozialkapitalbegriffs, vorzugsweise durch die Verknüpfung dieses Vorhabens mit empirischen Arbeiten. Hierzu gehören auch der weitere Ausbau unserer Taxonomie der verschiedenen Arten von Sozialkapital sowie die Identifizierung der Analysefelder und Arten von Sozialkapital, bei denen die Verwendung dieses Konzepts am sinnvollsten ist.

5. Entwicklung besserer Messgrößen für das in den einzelnen sozialen Gruppen vorhandene Sozialkapital sowie, separat, aber dennoch in Verbindung damit, Verbesserung der Messgrößen, die den Zugang des Einzelnen zu Sozialkapital erfassen. Zu diesem Zweck werden derzeit mehrere Erhebungsinstrumente erarbeitet. Die in Putnam (2000a) verwendeten Messgrößen beziehen sich auf Verhaltensweisen oder Ein- 
Es muss zudem aufgezeigt werden, welche Maßnahmen greifen.

Die neue Debatte über die Rolle des Sozialkapitals könnte mit der Debatte über das Humankapital vergleichbar sein, zu der die OECD in den sechziger Jahren einen bedeutenden Beitrag geleistet hat.

Die OECD könnte auch hier weitere Beiträge leisten. stellungen, die in Erhebungen bei Einzelpersonen erfasst wurden. Die von den Befragten angegebenen Dispositionen in Bezug auf das Vertrauen bzw. die Angaben bezüglich Aktivitäten im Zusammenhang mit der informellen Sozialisierung, mit Wahlverhalten, Mitgliedschaft in verschiedenen Arten von Organisationen und freiwilliger Mitarbeit bei gemeinnützigen Vorhaben liefern wichtige Indikatoren für Normen und Muster des Netzwerkverhaltens der Bevölkerung insgesamt.

6. Durch Politikevaluierung, Gewinnung eines besseren Verständnisses der Maßnahmen, die sich bei der Förderung des Sozialkapitals als erfolgreich erwiesen haben, sowie der Zusammenhänge, in denen die Förderung des Sozialkapitals die größten Nutzeffekte bringt. Anregungen für Bereiche, die sich für eine Evaluierung anbieten, wurden bereits weiter oben gegeben.

\subsection{Schlussbetrachtungen}

In den sechziger Jahren hat die OECD einen großen Beitrag zur Definition und Anerkennung des Humankapitalkonzepts in der Wirtschaftswissenschaft geleistet. Eine Generation später steht der Begriff des Sozialkapitals in der Diskussion, und nach wie vor sind Unklarheiten in Bezug auf die Interpretation dieses Konzepts, Unsicherheiten in Bezug auf die Forschungsergebnisse und gravierende Messprobleme vorhanden. Der vorliegende Bericht enthält eine Analyse der neuesten Untersuchungsergebnisse zum Humankapital und stellt zugleich den Versuch dar, unsere Kenntnisse über das Sozialkapital zu erweitern.

Weltweit sind zurzeit breit angelegte Forschungsprojekte, Analysen und auf die Konzipierung einschlägiger Maßnahmen in Bezug auf das Humankapital ausgerichtete Arbeiten im Gange, und dies gilt auch zunehmend für den Bereich des Sozialkapitals. Es wird äußerst wichtig sein, den Überblick über all diese Aktivitäten zu behalten und Erfahrungen sowohl über die Politikkonsequenzen als auch über Messverfahren auszutauschen, so dass die wichtigsten Schlussfolgerungen herauskristallisiert werden können. Die Aufgabe der OECD wird darin bestehen, die Bereiche zu identifizieren, in denen ihre eigenen Beiträge von besonderem Nutzen sein können. Dies könnte in den folgenden drei Bereichen der Fall sein:

- Die OECD hat eine wichtige Rolle dabei gespielt, international vergleichbare Indikatoren über die Humankapitalbildung zusammenzustellen und auf der Basis dieser Indikatoren eine Politikanalyse vorzunehmen. Sie kann die entsprechenden Arbeiten fortsetzen und durch eine zusätzliche Analyse potentiell komplementärer Funktionen des Human- und Sozialkapitals vertiefen.

- Es besteht von Seiten der Entscheidungsträger und der Politikverantwortlichen ein zunehmendes Interesse am Sozialkapital, doch gibt es nur wenig eindeutige Beispiele, in denen die Ergebnisse der Forschungsarbeiten über Sozialkapital vorbehaltlos angewandt wurden. Das Konzept ist nach wie vor relativ vage, und im Allgemeinen wird keine einheitliche Terminologie verwendet. Hinzu kommt, dass der Begriff Sozialkapital in vielen verschiedenen Kontexten verwendet 
Die besondere Berücksichtigung von Humanund Sozialkapital wird dazu beitragen, das Wohlergehen künftiger Generation zu wahren. wird, und es steht bislang nicht fest, in welchem Bereich die Verwendung des Konzepts die größten Nutzeffekte bringen wird. Die Effektivität der verschiedenen Maßnahmen im Hinblick auf die Förderung des Sozialkapitals wurde zudem bislang so gut wie noch gar nicht erforscht. Dies legt den Schluss nahe, dass es sinnvoll wäre, einen hochrangigen OECD-Workshop zu veranstalten, der die Aufgabe haben würde, die Erfahrungen und theoretischen Ansätze der Mitgliedsländer auszutauschen und den jüngsten Forschungsergebnissen über Sozialkapital gegenüberzustellen.

- Entscheidend ist eine Verbesserung der Messgrößen für Sozialkapital, und in mehreren Ländern wird zurzeit an der Entwicklung von entsprechenden Erhebungsinstrumenten gearbeitet. Eine Option könnte für die OECD darin bestehen, die Möglichkeit einer Bündelung der internationalen Ressourcen in diesem Bereich zu untersuchen - etwa durch die Entwicklung eines gemeinsamen Erhebungsinstruments in Verbindung mit den laufenden OECD-Arbeiten über Bildungsindikatoren. So könnte es sich z.B. lohnen, die Möglichkeit zu untersuchen, die Messung von Sozial- und Humankapital im Rahmen einer Erhebung über die Qualifikationen von Erwachsenen miteinander zu verknüpfen. Mit einem derartigen Instrument wäre es im Prinzip möglich, die gemeinsame und relative Wirkung von Sozial- und Humankapital auf die Lebenschancen des Einzelnen zu beurteilen.

Die Gewährleistung eines anhaltenden Wirtschaftswachstums und der Verbesserung des Wohlergehens aller ist eine der größten Herausforderungen für die OECD-Länder. In einer sich rasch verändernden globalen Wirtschaft ist der Wert der sozialen und humanen Fähigkeiten für die Meisterung dieser Aufgabe wichtiger denn je. Unsere Aufgabe besteht nunmehr darin, zu einem besseren Verständnis der Rolle dieser Fähigkeiten - des Human- und des Sozialkapitals - zu gelangen. Denn dieses Verständnis ermöglicht es uns, die menschlichen Kompetenzen besser zu nutzen und neue Wege der Zusammenarbeit zu finden, um das Wohlergehen aller zu erhöhen - und künftigen Generationen mindestens die Chancen zu eröffnen, die wir selbst hatten. 


\section{Anmerkungen}

1. Vgl. OECD (2001, Kapitel 4) wegen einer Erörterung des Problems der Definition neuer Qualifikationen, die für die wissensbasierte Wirtschaft erforderlich sind.

2. Epstein (1995) liefert eine Vision dessen, wie eine Partnerschaft zwischen Schule, Familie und Gemeinschaft funktionieren könnte: Die Schule könne mehr die Rolle einer Familie übernehmen, d.h. nicht nur die Kinder, sondern auch deren Familien einbeziehen, und die Familie könne insofern in verstärktem Maße die Funktion einer Schule übernehmen, als sie ein dem Lernen förderliches Klima schafft.

3. Beispiele für diese elektronischen Netzwerke wären z.B. elektronische „schwarze Bretter“ auf lokaler Ebene, die dazu dienen, Informationen über Stellenangebote, freiwillige Hilfsangebote usw. zu verbreiten.

4. Vgl. Abschnitt 3.7 wegen der Befunde über die Auswirkungen der sozialen Bindungen und die Qualität der persönlichen Beziehungen. 


\section{Anhang A}

\section{VERSCHIEDENE MESSGRÖSSEN FÜR DAS WOHLERGEHEN}

Erhebungen über die Meinung der Öffentlichkeit und die Lebensqualität in verschiedenen OECD-Ländern, namentlich die World Values Study, legen die Vermutung nahe, dass das globale Wohlergehen immer mehr hinter dem BIP-Wachstum zurückbleibt. Daraus folgert, dass die Erträge des Wirtschaftswachstums in den Industriestaaten heute möglicherweise rückläufig sind (Inglehart, 2000; Eckersley, 1998). Eine der hieraus resultierenden Schlussfolgerungen lautet, dass der Qualität des Wirtschaftswachstums künftig mehr Aufmerksamkeit geschenkt werden muss, und zwar unter Berücksichtigung aller anderen etwaigen Veränderungen der materiellen oder sozialen Umwelt, die mit dem Wirtschaftswachstum zusammenhängen und das Wohlergehen beeinflussen.

Das BIP und andere auf der Volkswirtschaftlichen Gesamtrechnung basierende Messgrößen (u.a. das „grüne“ BIP und die „echte Ersparnisbildung“) gehen von monetären Schätzungen der Einkommensströme sowie von den Berichtigungen der messbaren Bestände aus. Der Wert der Bestandsveränderungen bzw. der Preis einer gegebenen Tätigkeit werden auf der Basis dessen ermittelt, was die Verbraucher und die Gesellschaft zu zahlen gewillt sind. Nicht marktbestimmte Kosten und Nutzeffekte können nicht ohne weiteres in dieses System einbezogen werden.

In diesem Anhang wird eine Reihe von Messgrößen beschrieben, die herangezogen wurden, um gewisse Aspekte des Wohlergehens in verschiedenen Ländern bzw. im Zeitverlauf miteinander zu vergleichen. Bei jedem dieser Beispiele geht es um Aspekte des Wohlergehens, die bei den Messgrößen auf der Basis des BIP oder ähnlicher Indikatoren nicht berücksichtigt werden. Eine gewisse Vorstellung von der vermutlichen Größenordnung der Anpassungen des BIP bzw. der Investitionen vermitteln einige der weiter unten beschriebenen Studien. Unter Verwendung von Daten von 1965 für die Vereinigten Staaten kamen Nordhaus und Tobin (1972) zu dem Ergebnis, dass der Wert der Freizeit und anderer nicht im BIP erfasster Aktivitäten annähernd 100\% bzw. 50\% des BIP der USA ausmachte. Jorgenson und Fraumeni (1987), die mit Daten für die USA von 1982 arbeiteten, schätzen die Humankapitalinvestitionen auf über $50 \%$ des gesamten privaten Bruttoinlandsprodukts.

Wenn auch die Ansätze zur Messung des Wohlergehens vielfach relativ neu sind, trifft dies doch nicht auf die ihnen zugrunde liegenden Überlegungen zu. Nordhaus und Tobin (1972) schlugen einen zusammengesetzten Indikator für die Nettogröße des wirtschaftlichen Wohlergehens vor, bei dem zusätzliche Komponenten in eine weiter gefasste Messgröße für nachhaltigen Konsum und nachhaltiges Wohlergehen einbezogen werden. So ergänzten sie das BIP um folgende Faktoren:

- geschätzter Wert bestimmter Aktivitäten, wie z.B. Produktion der privaten Haushalte in Form von Kinderbetreuung und sonstigen Dienstleistungen, sowie Wert der Freizeit ${ }^{1}$;

- Sachkapitalinvestitionen der öffentlichen und privaten Haushalte (z.B. Gebrauchsgüter im Fall der privaten Haushalte).

Ausgeklammert aus dem BIP wurden hingegen folgende Elemente:

- instrumentale bzw. intermediäre Ausgaben für Aktivitäten, die, wie es bei den Autoren heißt, für sich genommen ganz offensichtlich keine unmittelbare Quelle des Wohlergehens sind, jedoch leider notwendige Inputs für Aktivitäten darstellen, die ihrerseits Wohlergehen erzeugen können (a.a.O., S. 7) (z.B. Kosten des Pendelverkehrs zwischen Arbeitsplatz und Wohnort bzw. öffentliche Ausgaben für Polizei, Abwasser- und Müllentsorgung, Instandhaltung von Straßen und Landesverteidigung);

- Kapitalabschreibungen sowie Investitionen zur Befriedigung künftiger Wachstumserfordernisse ${ }^{2}$;

- Unannehmlichkeiten des Lebens in der $\operatorname{Stadt}^{3}$ (z.B. Kosten von Umweltverschmutzung, Abfallentsorgung, Verkehrsstaus und Lärm); 
- Bildung und Gesundheit (mit der Begründung, dass es sich um Vorleistungen handelt, deren positive Effekte bereits in Einkommensaufbesserungen zum Ausdruck kommen).

Anders als Nordhaus und Tobin schlossen Jorgenson und Fraumeni (1987) Bildungsinvestitionen in ihre Messgröße des Wohlergehens ein. Ebenso wie Nordhaus und Tobin nahmen sie jedoch Berichtigungen um Subventionen, Kapital- und Konsumausgaben der privaten Haushalte sowie die private Freizeitproduktion vor. Der Wert der Bildungsinvestitionen wurde geschätzt als Summe des Gegenwartswerts der lebenslangen Einkommen sämtlicher Individuen zuzüglich des rechnerischen Werts des Einkommens, das den Studierenden entgeht ${ }^{4}$. Es wurden keine Abzüge auf Grund von Kindererziehungs- oder Bildungskosten vorgenommen. Demgegenüber schloss Kendrick (1976) Schätzungen der Ausbildungskosten, der von den Arbeitgebern getragenen Fort- und Weiterbildungskosten, der Kosten für informelles Lernen (mittels Bibliotheken, Museen und Druckmedien) wie auch den Wert der Forschungs- und Entwicklungsausgaben ein.

Die meisten Messgrößen sind zusammengesetzte und z.T. nichtmonetäre Indikatoren. Andere beinhalten auf monetärer Basis vorgenommene Schätzungen verschiedener sozialer Trends bzw. Ergebnisse und gehen von deren Integration in einen Rahmen der Volkswirtschaftlichen Gesamtrechnung aus, sei es unmittelbar oder mittels Satellitenkonten. Es lassen sich u.a. folgende Beispiele für zusammengesetzte Indikatoren des Wohlergehens nennen:

- Beim Genuine Progress Indicator (GPI) handelt es sich um eine Messgröße des Wohlergehens auf monetärer Basis, der bis zum Jahr 1950 zurückreichende Daten zugrunde liegen ${ }^{5}$. Der Indikator erweitert den üblichen volkswirtschaftlichen Erfassungsrahmen, der die im klassischen Sinne gemessene wirtschaftliche Produktion abdeckt, um den ökonomischen Beitrag von Familien und Gemeinschaften wie auch um jenen des natürlichen Habitats.

- Der Human Development Index der Vereinten Nationen (UNHDI) misst die globale Leistung eines Landes im Hinblick auf drei grundlegende Dimensionen der menschlichen Entwicklung - Lebensdauer, Wissen und ökonomische Ressourcen. Er wird gemessen an der Lebenserwartung und am Niveau der Bildungsabschlüsse (elementare Grundqualifikationen Erwachsener und kombinierte Schulbesuchsquote für den Primar-, Sekundar- und Tertiärbereich) und wird bereinigt um das Pro-Kopf-Einkommen in konstanten Kaufkraftparitäten (UNDP, 1990, 2000).

- In Kanada wurde ein Index of Social Health (ISH) auf der Basis des Fordham-Indexes für gesunde soziale Bindungen $^{6}$ entwickelt. Dieser Index wurde mit Hilfe einer Reihe sozioökonomischer Indikatoren konstruiert und misst die Fortschritte gegenüber den für die jeweils untersuchten Länder früher beobachteten Ergebnissen ${ }^{7}$.

- Der Index of Sustainable Economic Welfare (ISEW) misst verschiedene Aktivitäten, die zur Lebensqualität beitragen. Die durch ökonomische Aktivitäten verursachte Luftverschmutzung wird in Abzug gebracht, der Wert unbezahlter Hausarbeit hingegen eingeschlossen. Dieser Index deckt auch Bereiche wie Einkommensungleichheiten, sonstige Umweltschäden und Verbrauch von Umweltgütern ab. Der ISEW wurde bislang für folgende Länder berechnet: Vereinigtes Königreich, Deutschland, Österreich, Schweden, Niederlande und Italien sowie Vereinigte Staaten.

Der Index of Economic Well-being wurde von Osberg (1985) entwickelt und später dann ebenfalls in Osberg (2001) mit Hilfe von Daten für die Vereinigten Staaten, das Vereinigte Königreich, Kanada, Australien, Norwegen und Schweden für 1980-1996 näher erläutert und illustriert. Osberg zufolge können gerade die bei den BIPBerechnungen ausgesparten Elemente im Hinblick auf das Sozialkapital besonders relevant sein. Der Index beruht auf vier Komponenten:

- Effektive Pro-Kopf-Konsumströme, wozu der Konsum marktbestimmter und nicht marktbestimmter Güter und Dienstleistungen, Veränderungen der Lebenserwartung wie auch der Freizeit zählen (Pro-Kopf-Konsum marktbestimmter Güter und Dienstleistungen, öffentliche Pro-Kopf-Ausgaben, Veränderungen der Arbeitszeit).

- Gesellschaftliche Netto-Akkumulation von Vorräten produktiver Ressourcen, einschließlich der Pro-KopfNetto-Akkumulation von Sach- und Immobilienkapital sowie Netto-Akkumulation von Humankapital (jährliche Bildungsausgaben, bezogen auf die gesamte erwachsene Bevölkerung) und Pro-Kopf-FuE-Ausgaben, abzüglich der Nettoveränderung des Niveaus der Auslandsverschuldung und der gesamtwirtschaftlichen Kosten der Umweltzerstörung. 
- Einkommensverteilung gemäß dem Gini-Index der Ungleichheit (Einkommensungleichheit nach Steuern) sowie Grad und Ausmaß der Armut.

- Wirtschaftliche Sicherheit in Bezug auf Arbeitslosigkeit, Gesundheitsprobleme, Armut von Alleinerziehenden und Armut im Alter.

Die verschiedenen Komponenten dieses Index sind in Abbildung A.1 dargestellt. Für die Kombination der vier wesentlichen Elemente (Durchschnittskonsum, Generationenvermächtnis, Ungleichheit/Armut und wirtschaftliche Unsicherheit) können, je nach den Wertvorstellungen und subjektiven Einschätzungen der Nutzer, unterschiedliche Gewichtungen verwendet werden. Ein wesentlicher Aspekt besteht bei diesem Index darin, Verteilungsfragen, sei es in Bezug auf die Mittelaufteilung zwischen laufendem und künftigem Gesamtverbrauch, sei es bezüglich der Verteilung von Einkommen und wirtschaftlichen Chancen auf die verschiedenen Teilgruppen im jeweils untersuchten Zeitraum, eine spezielle Gewichtung beizumessen. Da die vier Hauptkomponenten unabhängig voneinander ermittelt wurden, bereitet die Durchführung von Sensitivitätsanalysen zur Feststellung des Effekts, den die unterschiedlichen Gewichtungen auf die beobachteten Gesamttendenzen haben, keine Schwierigkeiten. Die Darstellung in Abbildung A.2 veranschaulichen die Ergebnisse, die mit der „Standard“- bzw. der ,alternativen“ Gewichtung für die folgenden fünf Länder erzielt wurden: Australien, Kanada, Schweden, Vereinigtes Königreich und Vereinigte Staaten.

Die auf diese Weise erzielten Ergebnisse zeigen, dass das Pro-Kopf-BIP-Wachstum in allen untersuchten Ländern stärker ist als das des materiellen Wohlergehens, wobei der Abstand allerdings je nach Land unterschiedlich ist. In allen Ländern waren negative Tendenzen bei den Variablen Ungleichheit/Armut und Unsicherheit ein Zeichen dafür, dass das materielle Wohlergehen tendenziell hinter dem BIP-Wachstum zurückblieb. In den Vereinigten Staaten stieg das Pro-Kopf-BIP im Zeitraum 1980-1997 um ungefähr 30\%, während der Index des materiellen Wohlergehens fast konstant blieb (so erhöhte er sich im selben Zeitraum lediglich um 4\%). Im Vereinigten Königreich ist das Pro-Kopf-BIP sogar noch stärker gewachsen (um 40\%), während der IEW gleichzeitig um fast 10\% sank. Osberg stellt fest, dass diese Ergebnisse die deutliche Zunahme der wirtschaftlichen Ungleichheiten im selben Zeitraum widerspiegeln. Im Falle der Vereinigten Staaten ist als zusätzlicher Faktor die beträchtliche Verlängerung der Arbeitszeit zu nennen. Wie Osberg berichtet, spielt beim Rückgang des IEW für das Vereinigte Königreich und Schweden die relative Gewichtung des laufenden Verbrauchs im Vergleich zu Einkommensverteilung und wirtschaftlicher Unsicherheit eine wichtige Rolle. Im Falle Australiens und Kanadas mit ihrer größeren Abhängigkeit von der Rohstofferzeugung sind beim Pro-Kopf-BIP stärkere Schwankungen als beim IEW-Wert festzustellen. Andere Gewichtungen für die wirtschaftliche Unsicherheit und Ungleichheit machen beim IEW-Wert für Kanada keinen größeren Unterschied.

Jeder Versuch einer Zusammenfassung dessen, was das Wohlergehen in komplexen und zunehmend diversifizierten Gesellschaften ausmacht, „,setzt zwangsläufig eine Reihe ethischer und statistischer Ermessensentscheidungen voraus“ (Osberg, 2001). Die verschiedenen vorstehend erörterten Ansätze stellen wichtige Schritte auf dem Weg zu besseren Messgrößen dar, doch sind jedem dieser Ansätze von folgenden Faktoren her mehr oder minder einschneidende Grenzen gezogen:

- Auswahl der relevanten Indikatoren bzw. Komponenten aus der umfassenden Liste von Faktoren, die eigentlich berücksichtigt werden müssten, um einen vollständigen Index des Wohlergehens zu erhalten;

- Gewichtung der verschiedenen Komponenten und Indikatoren;

- Interpretation der quantitativen Entwicklung dieser Elemente im Verhältnis zur BIP-Entwicklung, deren Komponenten weniger klar abzugrenzen sind (z.B. Arbeitslosigkeit).

Alles in allem kann diese Vielzahl von Indikatoren mit den jeweils willkürlichen Gewichtungen und Skalierungen zu endlosen Unstimmigkeiten über die Gewichtung der einzelnen Elemente des Wohlergehens führen. Die Leser werden ihre eigenen Schlussfolgerungen in Bezug auf die Frage ziehen müssen, ob einer dieser Ansätze eine zwar unvollkommene Alternative zum BIP darstellt, als Messgröße aber dennoch vorzuziehen ist. Die Schlüsselfrage lautet u.a., ob eine partielle Messgröße nicht letztlich besser ist als überhaupt keine kombinierte Messgröße, die die sonstigen Aspekte des Wohlergehens erfasst. 
Abbildung A.1 Veranschaulichung des Osberg-Indexes

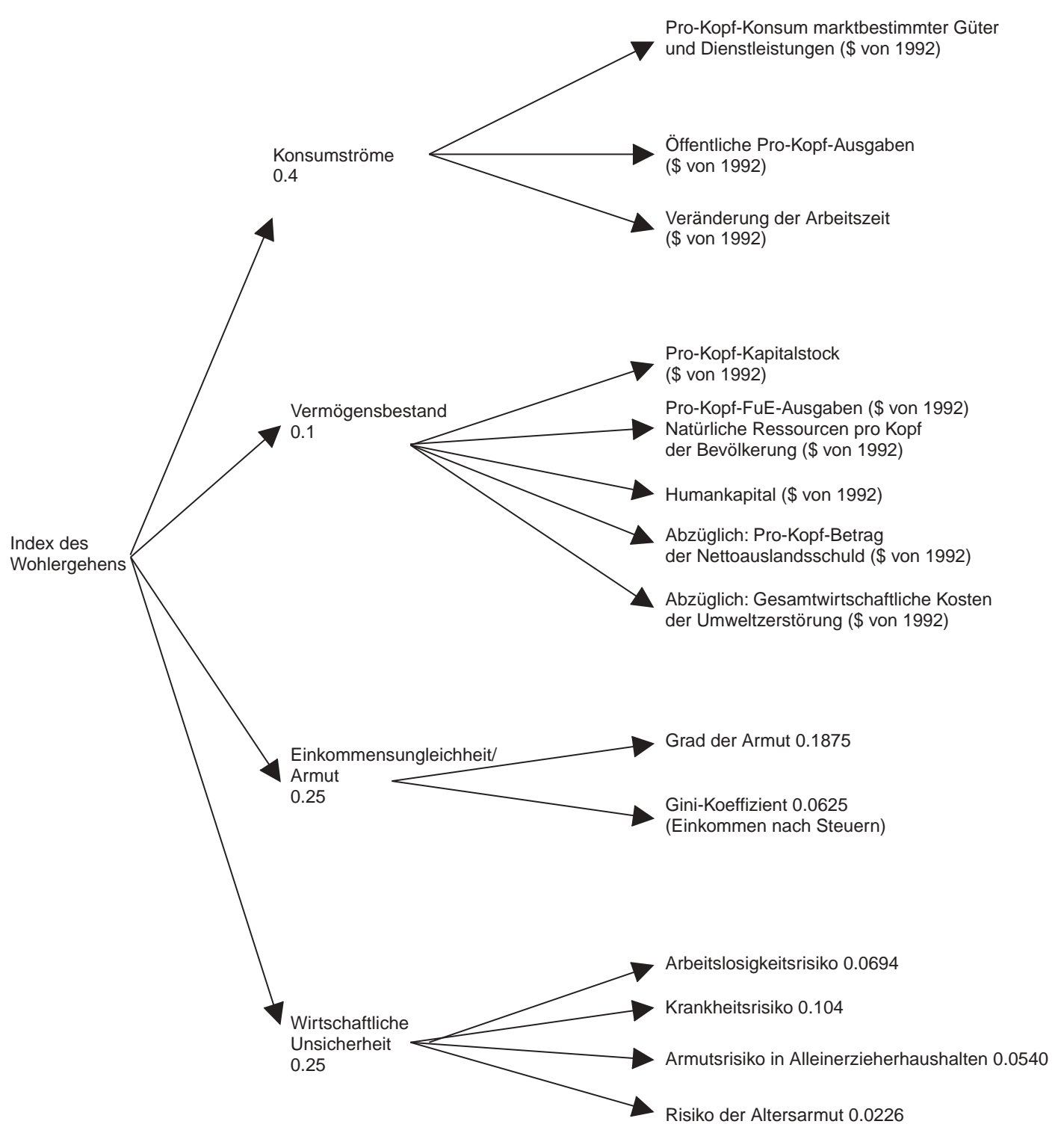

Quelle: Osberg (2001). 
Abbildung A.2 Trends des wirtschaftlichen Wohlergehens im internationalen Vergleich

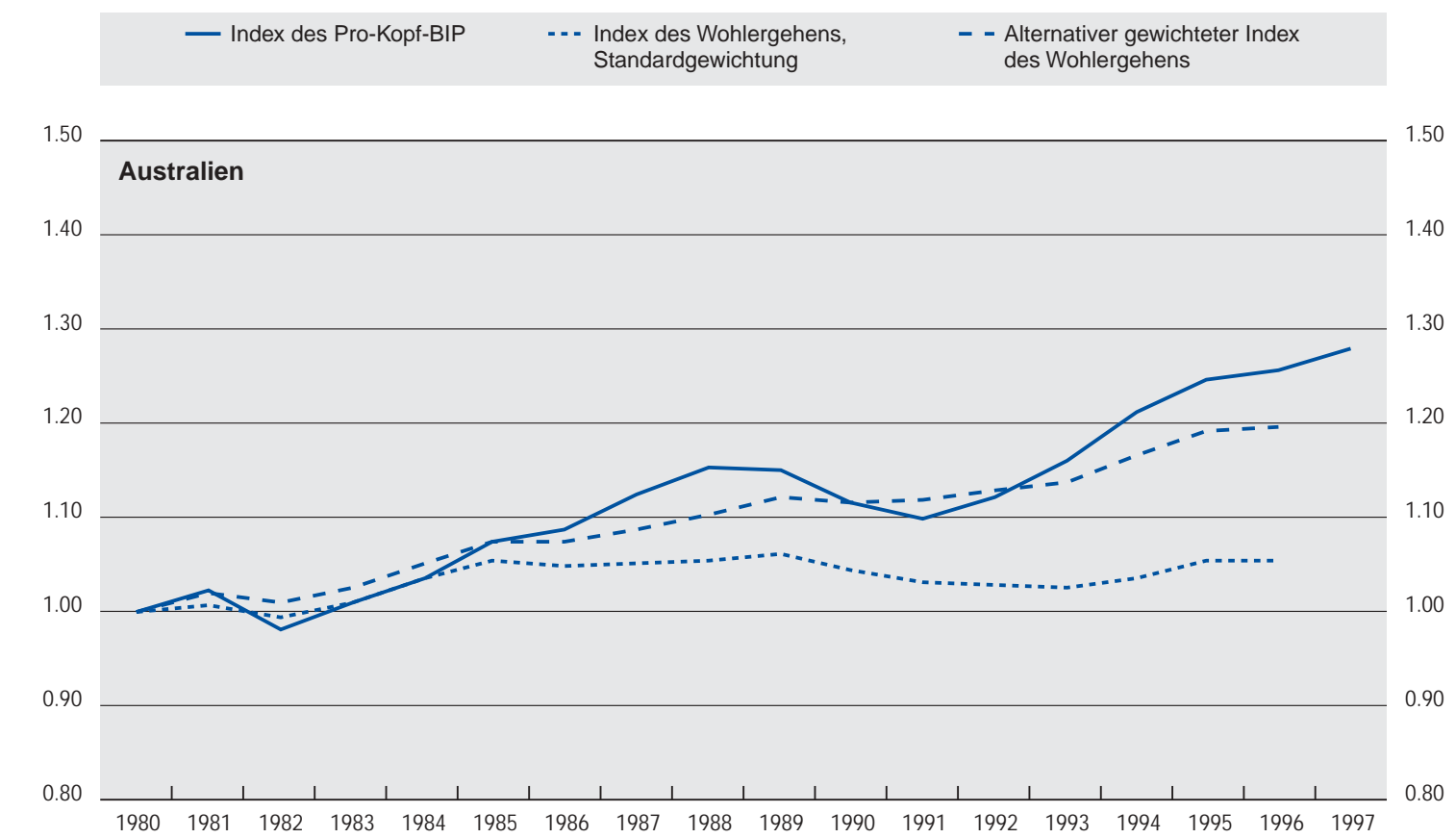

Quelle: Osberg (2001), Teil A.

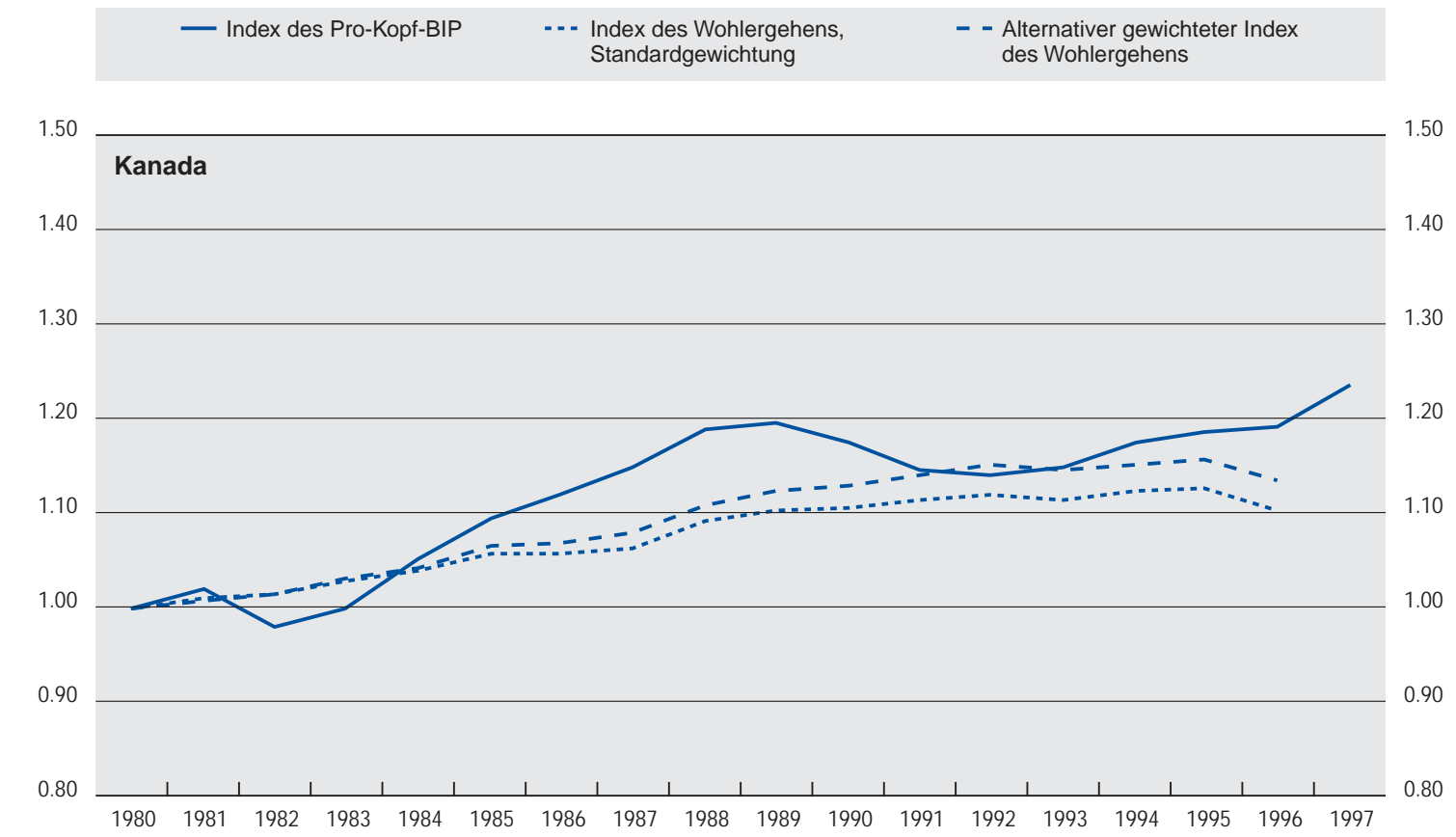

Quelle: Osberg (2001), Teil A. 
Abbildung A.2 (Forts.) Trends des wirtschaftlichen Wohlergehens im internationalen Vergleich

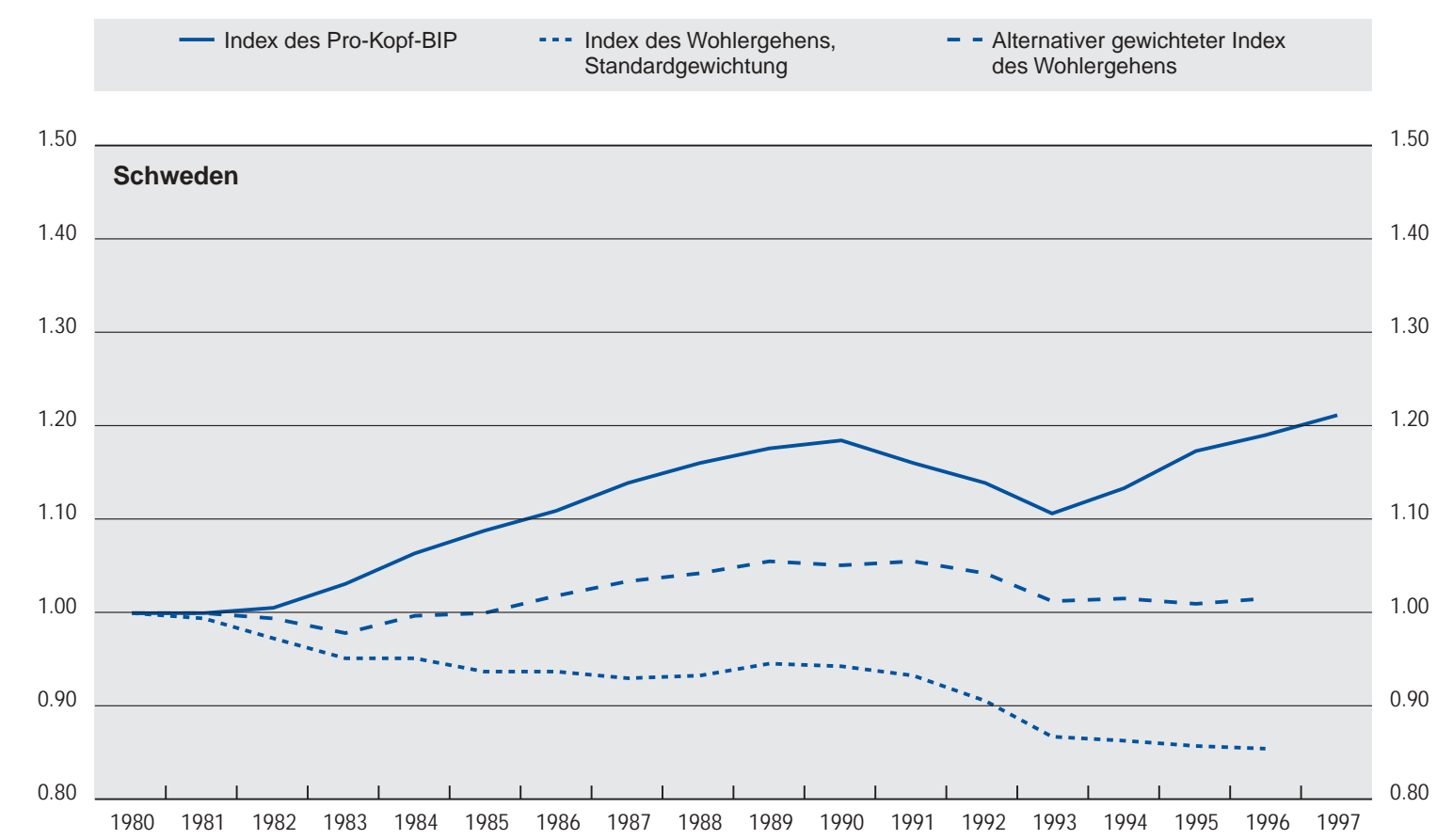

Quelle: Osberg (2001), Teil A.

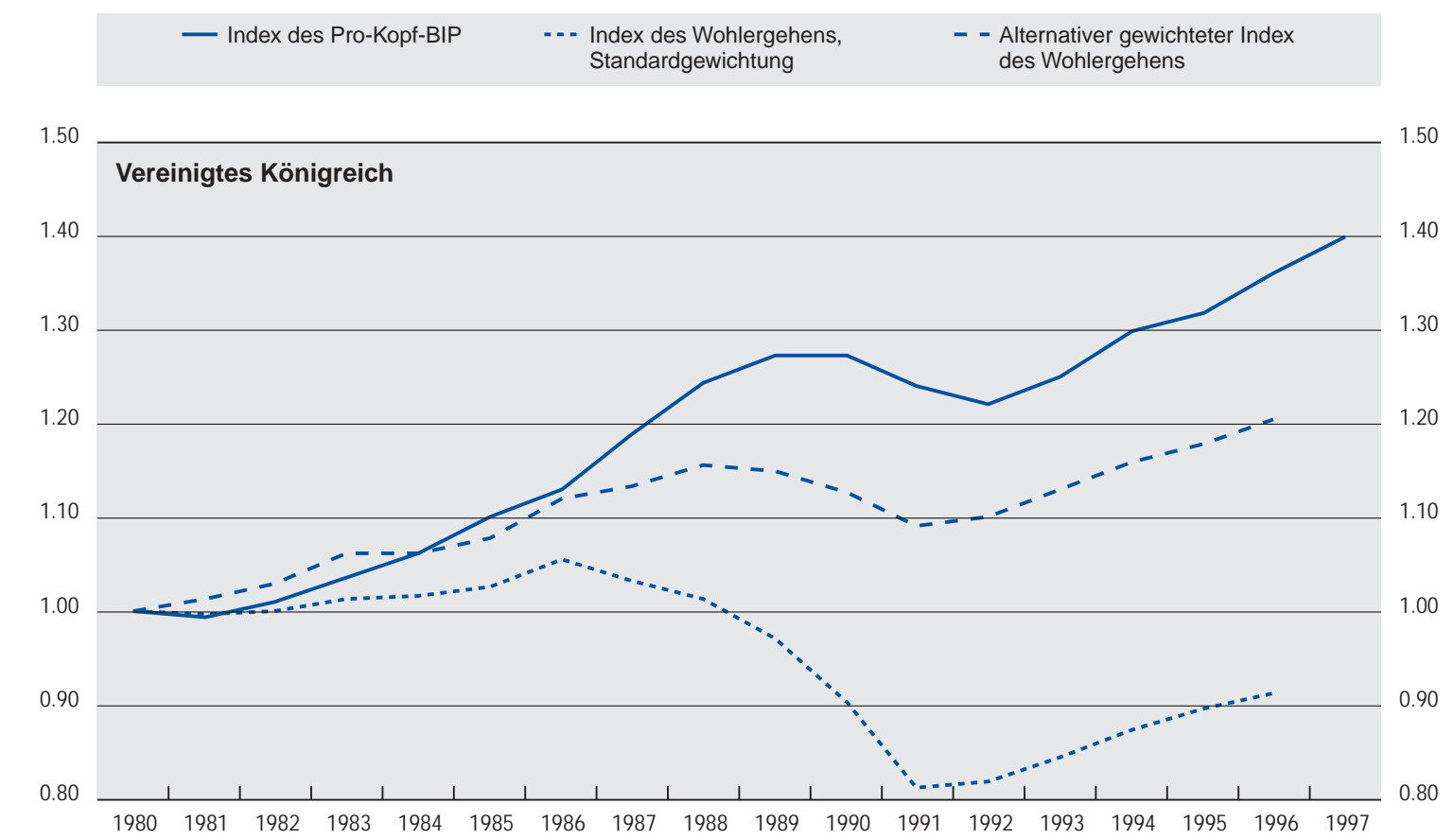

Quelle: Osberg (2001), Teil A. 
Abbildung A.2 (Forts.) Trends des wirtschaftlichen Wohlergehens im internationalen Vergleich

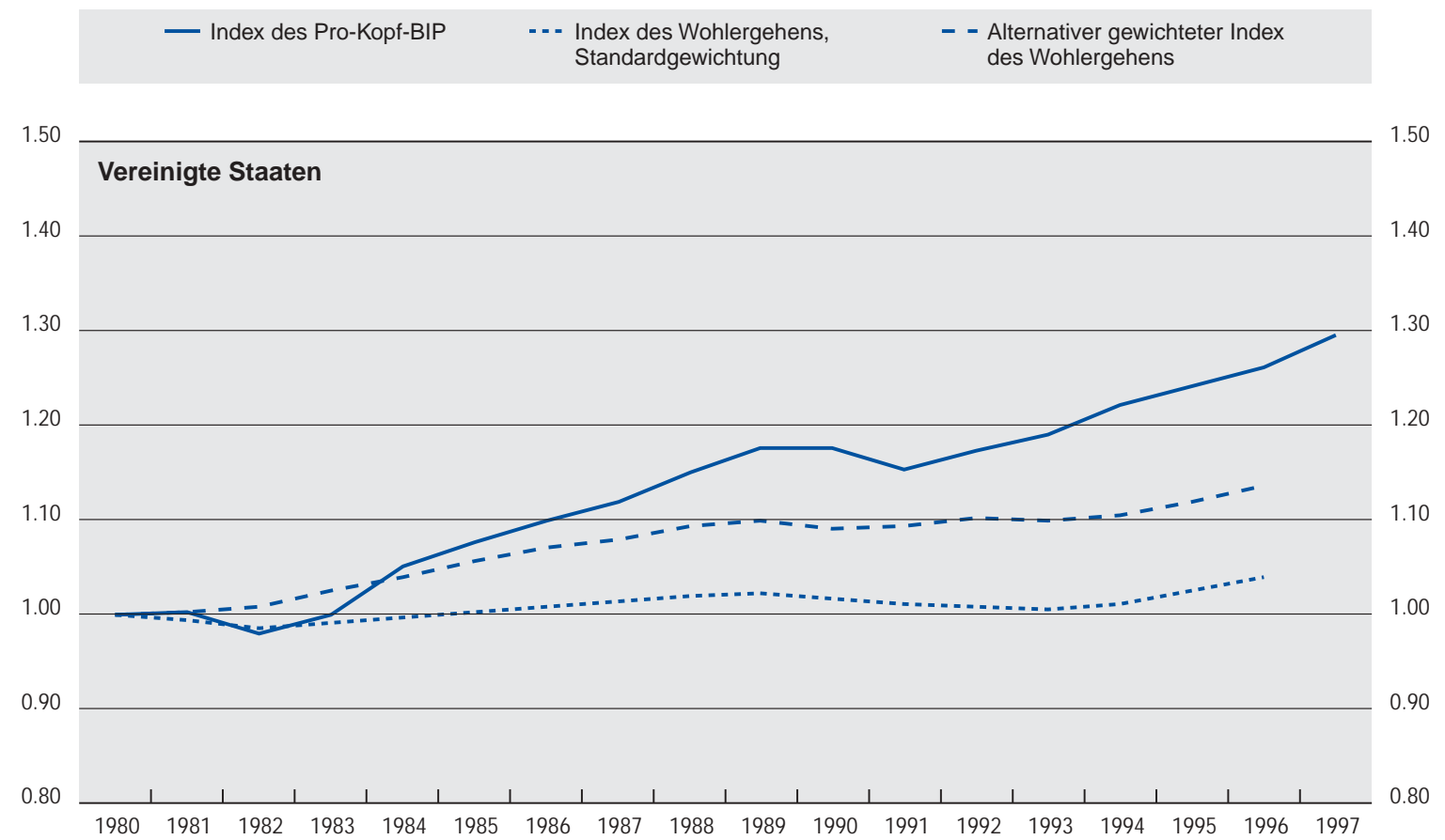

Quelle: Osberg (2001), Teil A. 


\section{Anmerkungen}

1. Der Freizeitwert wurde geschätzt, indem die geschätzte Zahl der Freizeitstunden für die Bevölkerung ab 14 Jahren mit den Tariflöhnen multipliziert wurde (was den Opportunitätskosten der Freizeit entspricht).

2. Von der Messung des wirtschaftlichen Wohlergehens ausgeklammert ist jener Teil der Sachinvestitionen, der zur Aufrechterhaltung eines konstanten Kapitalkoeffizienten erforderlich ist, wenn der Verbrauch um eine mit dem Bevölkerungswachstum und dem technischen Fortschritt kompatible Rate steigt.

3. Mangels hinreichender Daten wurde keine Berichtigung um die Effekte der Umweltzerstörung vorgenommen.

4. Der rechnerische Wert des Einkommensverlusts von Studierenden entspricht der Differenz zwischen den voraussichtlichen Arbeitsverdiensten der Erwerbspersonen mit und ohne Hochschulabschluss.

5. Mit dem Genuine-Progress-Indikator werden folgende Komponenten erfasst: privater Verbrauch, um Einkommensungleichheiten berichtigter privater Verbrauch, Wert von Hausarbeit sowie Kinderbetreuung und -erziehung durch die Eltern, Nettoauslandsguthaben bzw. -verbindlichkeiten, Verwendung des Kapitals der privaten Haushalte, Dienstleistungen im Zusammenhang mit Autobahnen und Straßen, Kosten der Unterbeschäftigung, Wert ehrenamtlicher Tätigkeiten, Nettokapitalausgaben, fortschreitende Erschöpfung nicht erneuerbarer Ressourcen, Kosten des Auseinanderbrechens von Familienverbänden, langfristige Umweltschäden, Kosten der Luftverschmutzung, Kosten für Gebrauchsgüter, Kosten der Wasserverschmutzung, Kosten des Pendelverkehrs, Kosten der Kriminalität, Verlust an Feuchtgebieten, Kosten der Lärmbelastung und des Ozonschichtabbaus, Kosten für Umweltschutzmaßnahmen der privaten Haushalte, Verlust an Freizeit.

6. Siehe auch: Index of Social Health (1995), Monitoring the Social Well-Being of the Nation, Fordham Institute for Innovation in Social Policy, Tarrytown, NY.

7. Der Index of Social Health (Brink und Zeesman, 1997) umfasst folgende Komponenten: Säuglingssterblichkeit, Kindesmissbrauch, Armut unter Kindern, Selbstmord Jugendlicher, Drogenmissbrauch, Abbruch der Sekundarschulausbildung, Arbeitslosigkeit, durchschnittlicher Wochenverdienst, Armut in der Altersgruppe ab 65 Jahren, aus eigener Tasche zu bestreitende Gesundheitsausgaben der Personen ab 65 Jahren, Straßenverkehrstote im Zusammenhang mit Alkoholmissbrauch, Tötungsdelikte, Zahl der Sozialhilfeempfänger, Schere zwischen Arm und Reich, Zugang zu erschwinglichem Wohnraum.

8. Zu den Komponenten des ISEW zählen u.a.: Konsumausgaben, Einkommensungleichheiten, Dienstleistungen in Verbindung mit Hausarbeit und Gebrauchsgütern, Dienstleistungen im Zusammenhang mit Autobahnen und Straßen, öffentliche Ausgaben für Gesundheit und Bildung, Kosten des Pendelverkehrs, Kosten für Umweltschutzmaßnahmen der privaten Haushalte, Kosten von Autounfällen, Kosten der Wasser-, Luft- und Lärmbelastung, Verlust an natürlichem Lebensraum, Verlust an landwirtschaftlicher Nutzfläche, fortschreitende Erschöpfung nicht erneuerbarer Ressourcen, Kosten der Klimaänderung und der Zerstörung der Ozonschicht, Bruttoinlandsprodukt (vgl. die Website Friends of the Earth: www.foe.org.uk/campaigns /sustainable_development/progress).

9. Osberg liefert ebenfalls Daten für acht weitere OECD-Länder, die z.T. allerdings weniger vollständig sind (vgl. Osberg, 2001). 
Anhang B

\section{WICHTIGE TRENDS BEI DEN SOZIALEN UND ÖKONOMISCHEN} RAHMENBEDINGUNGEN

Abbildung B.1 Reales Pro-Kopf-BIP, in konstanten Preisen, Durchschnitt auf der Basis ausgewählter OECD-Länder, 1966-1999

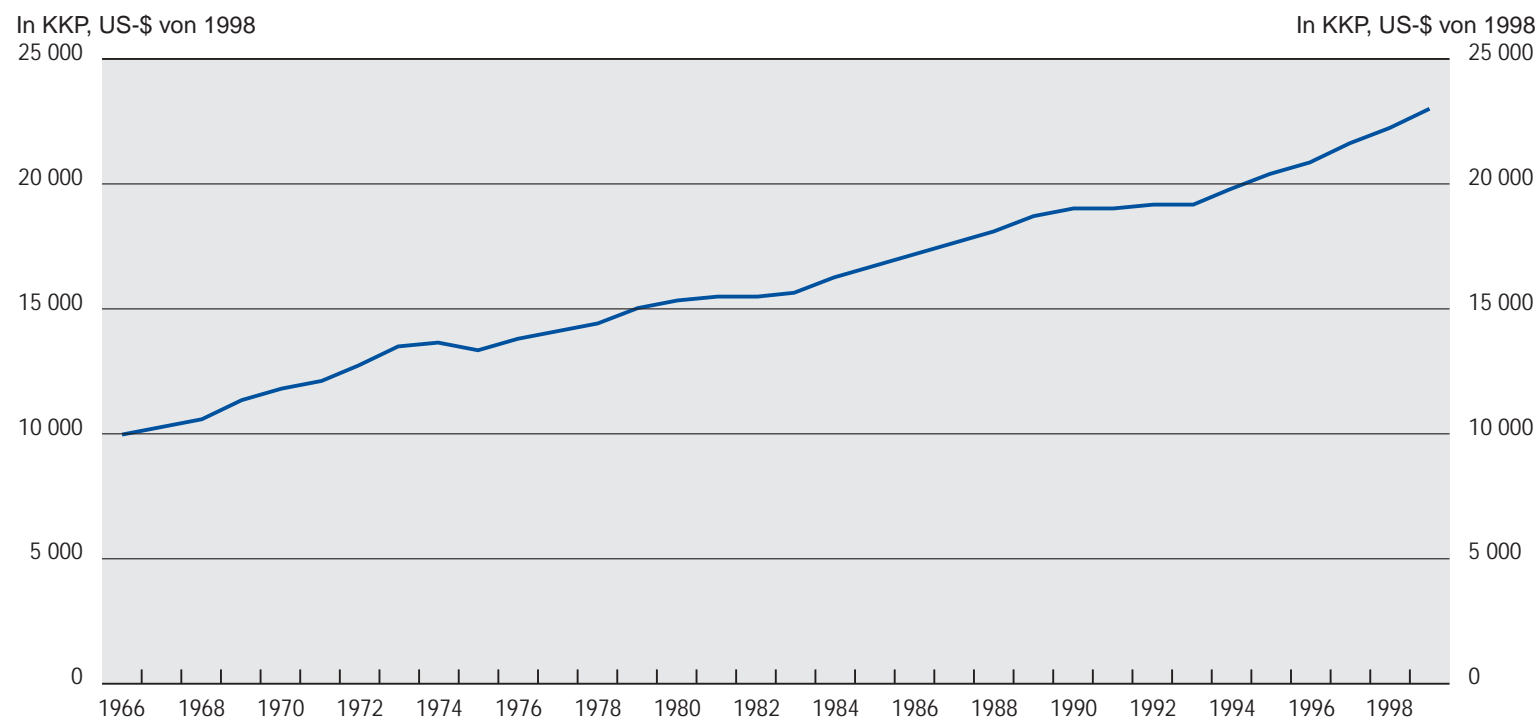

Die ausgewählten Länder sind: Australien, Belgien, Dänemark, Deutschland, Finnland, Frankreich, Griechenland, Irland, Island, Italien, Japan, Kanada, Luxemburg, Neuseeland, Niederlande, Norwegen, Österreich, Portugal, Schweden, Schweiz, Spanien, Vereinigtes Königreich, Vereinigte Staaten.

Quelle: OECD. Reales BIP, deflationiert unter Verwendung von US-\$ von 1998, auf der Basis von Kaufkraftparitäten. 
Abbildung B.2 Entwicklung der Einkommensungleichheit, für die gesamte Bevölkerung, in ausgewählten OECD-Ländern, Mitte der achtziger und neunziger Jahre

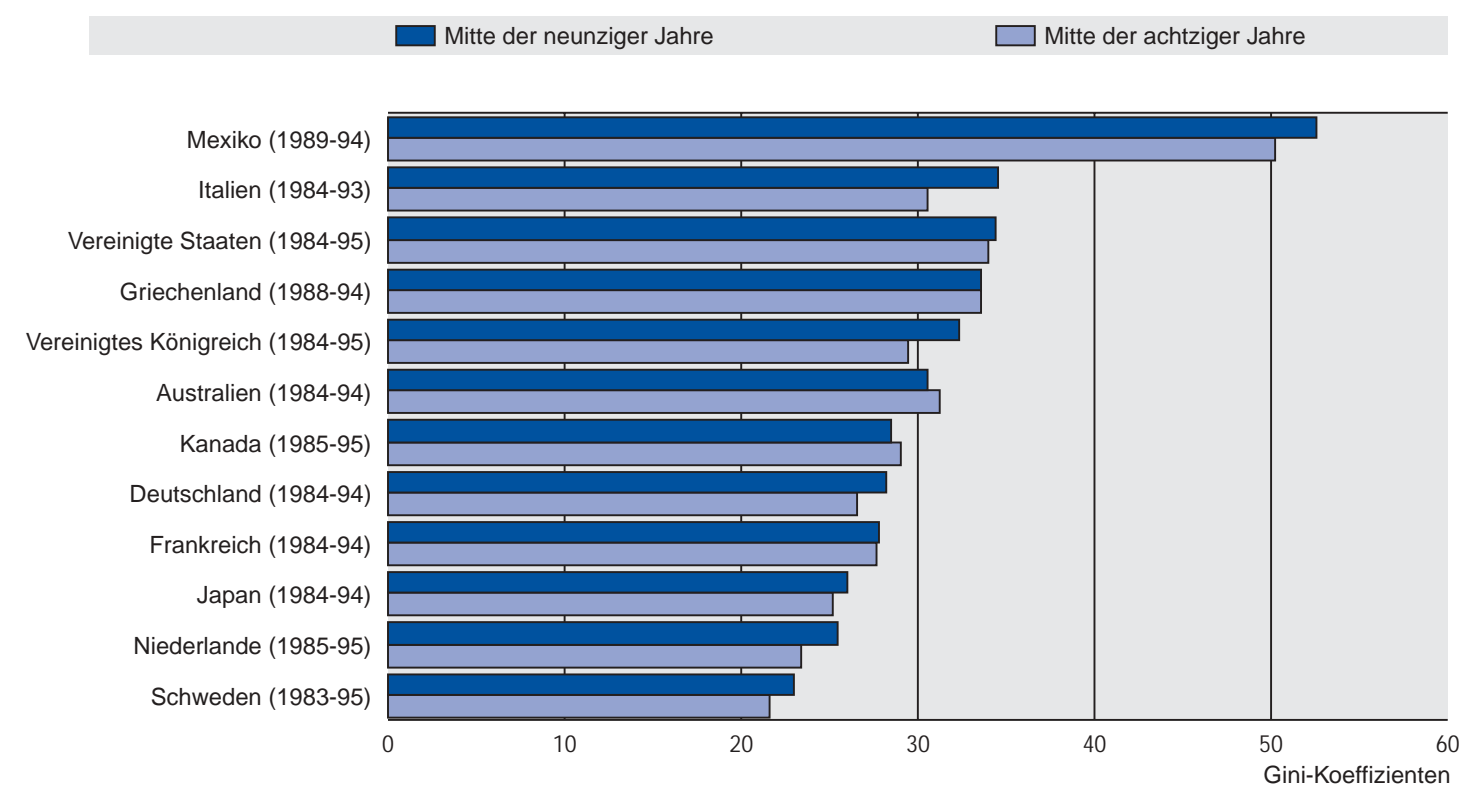

Anmerkung: Auf der Basis des verfügbaren Einkommens. Äquivalenz-Skalenelastizität $=0.5$.

Quelle: OECD (1999), ,Trends in Income Distribution and Poverty in the OECD Area“, unentgeltliches Dokument (lediglich in englischer Sprache erhältlich), Tabelle 2.2. 
Abbildung B.3 Prozentualer Anteil der in relativer Armut lebenden Kinder, in ausgewählten OECD-Ländern, neunziger Jahre

Prozentualer Anteil der in Haushalten mit weniger als 50\% des Medianeinkommens lebenden Kinder

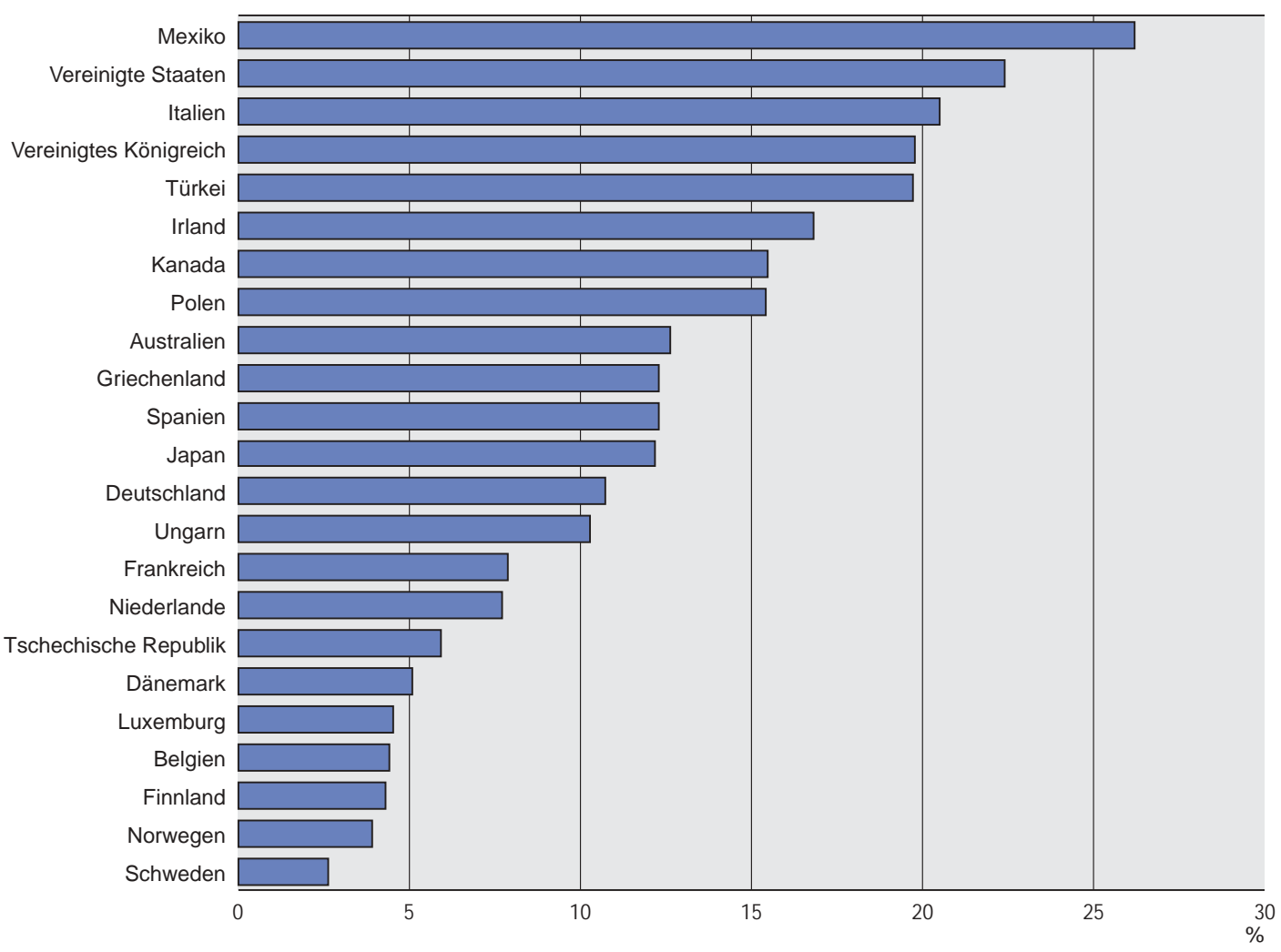

Anmerkung: Der prozentuale Anteil der in relativer Armut lebenden Kinder ist definiert als der Prozentsatz, der in Haushalten mit weniger als 50\% des Medianeinkommens lebt. Die Armutsquoten beziehen sich auf folgende Jahre: 1990 (Spanien), 1992 (Belgien, Dänemark und Japan), 1994 (Deutschland, Frankreich, Griechenland, Kanada, Luxemburg, Mexiko, Niederlande, Türkei, Ungarn), 1995 (Finnland, Italien, Norwegen, Polen, Schweden, Vereinigtes Königreich), 1996 (Tschechische Republik), 1996-1997 (Australien) und 1997 (Irland, Vereinigte Staaten).

Quelle: UNICEF (2000), „A league table of child poverty in rich nations“, Innocenti Report Card No. 1, UNICEF Innocenti Research Centre, Florenz. 
Abbildung B.4 Arbeitslosenquoten der 25- bis 54-Jährigen und der 15- bis 24-Jährigen, in ausgewählten OECD-Ländern, 1975-1999

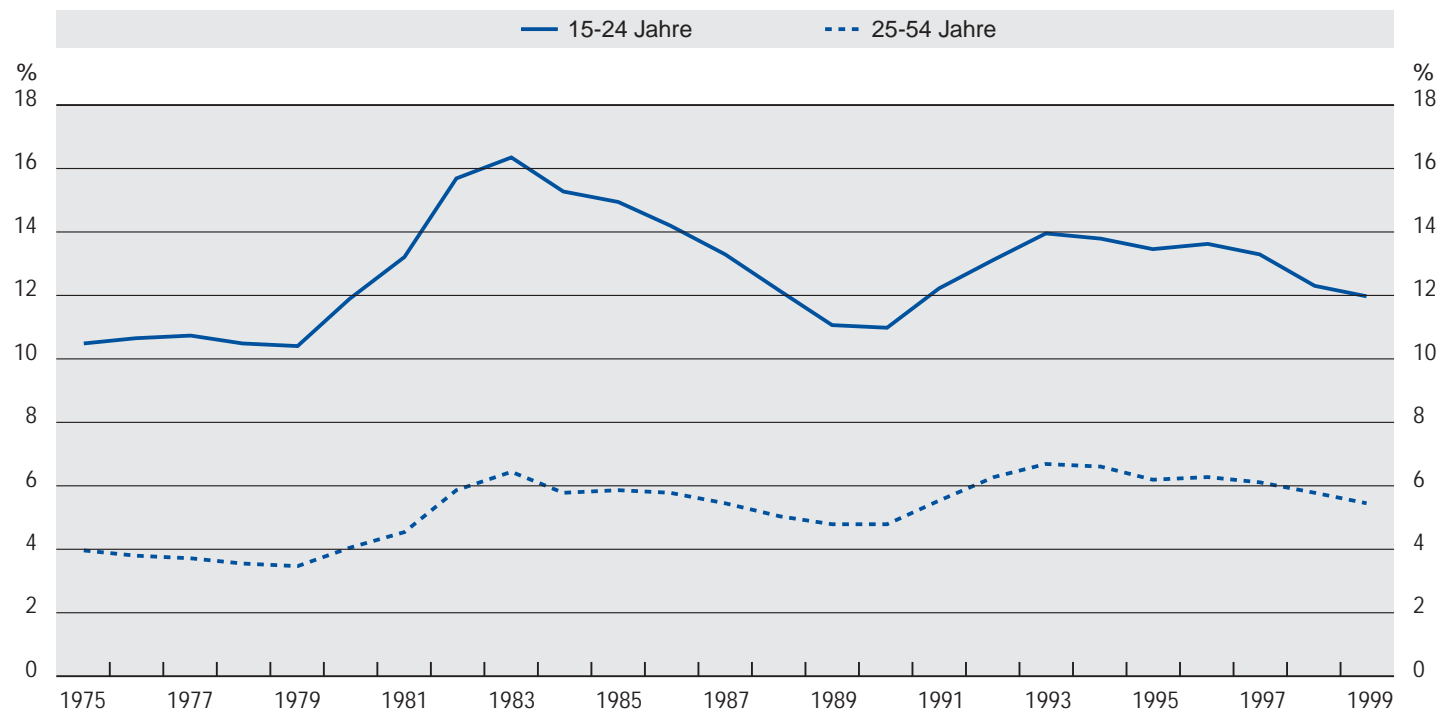

Die ausgewählten Länder sind: Australien, Deutschland, Finnland, Frankreich, Japan, Kanada, Niederlande, Norwegen, Portugal, Schweden, Spanien, Vereinigte Staaten.

Quelle: OECD (2000), auf der Basis von Arbeitsmarkterhebungen. Die durchschnittliche Arbeitslosenquote ist definiert als der durch die gesamte Erwerbspersonenzahl dividierte Prozentsatz der Arbeitslosen.

Abbildung B.5 Altenquotienten in den OECD-Ländern, 1950-2050

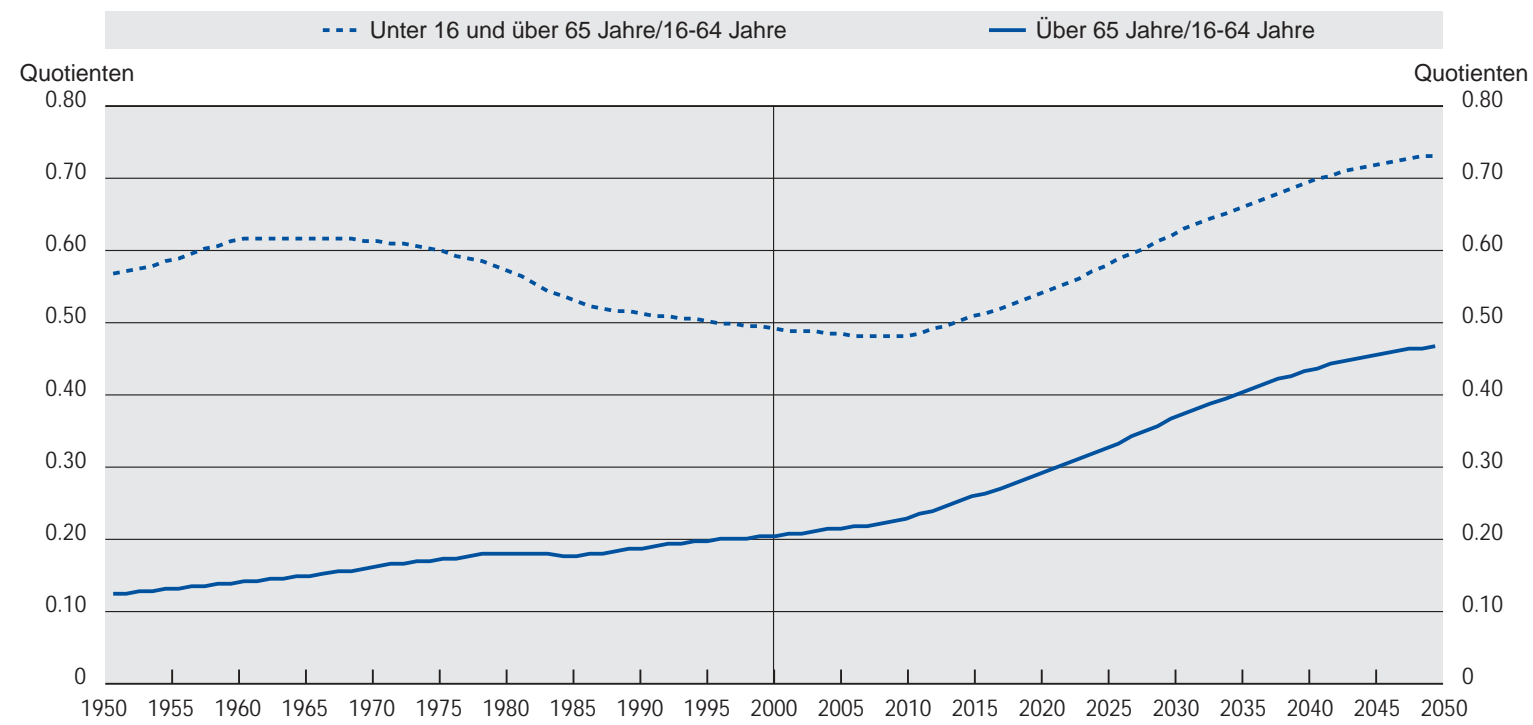

Altenquotienten: Die obere Linie veranschaulicht den Bevölkerungsanteil der unter 16- und über 65-Jährigen als prozentualen Anteil an der Erwerbsbevölkerung. Die untere Linie zeigt den prozentualen Anteil der über 65-Jährigen an der erwerbstätigen Bevölkerung.

Quelle: OECD (1999), A Caring World: The New Social Policy Agenda; Vereinte Nationen (Revision von 1998), World Population Prospects, 1950-2050, mittlere Variante. 
Abbildung B.6 Prozentualer Anteil der Bevölkerung der OECD-Länder im Alter von 25-64 Jahren mit Sekundar- oder Hochschulniveau, 1950-1998

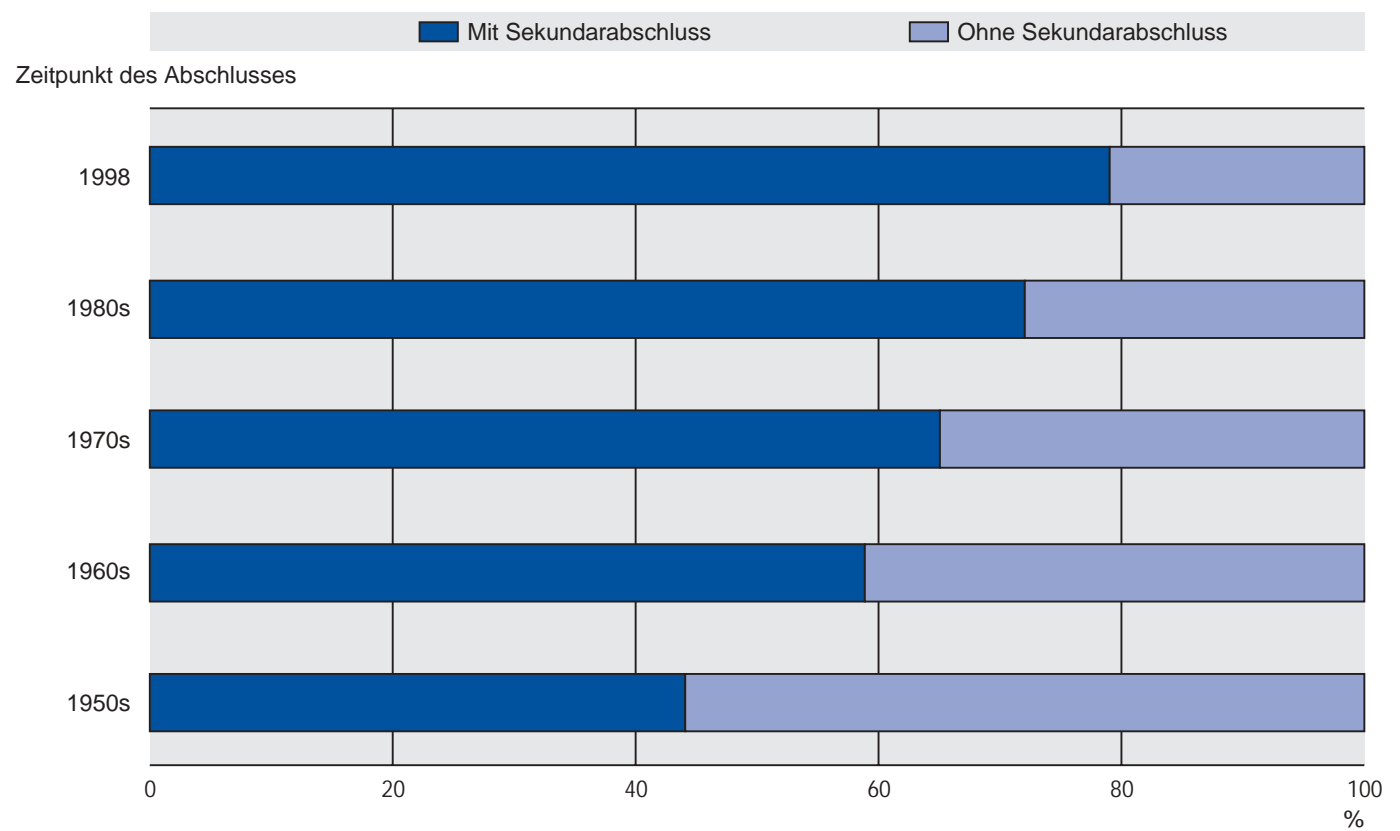

Quelle: OECD (2000), Bildung auf einen Blick-OECD-Indikatoren, Tabellen C2.2, C4.2.

Abbildung B.7 Inzidenzrate der Alleinerziehenden in ausgewählten OECD-Ländern, Vergleich zwischen den achtziger und neunziger Jahren

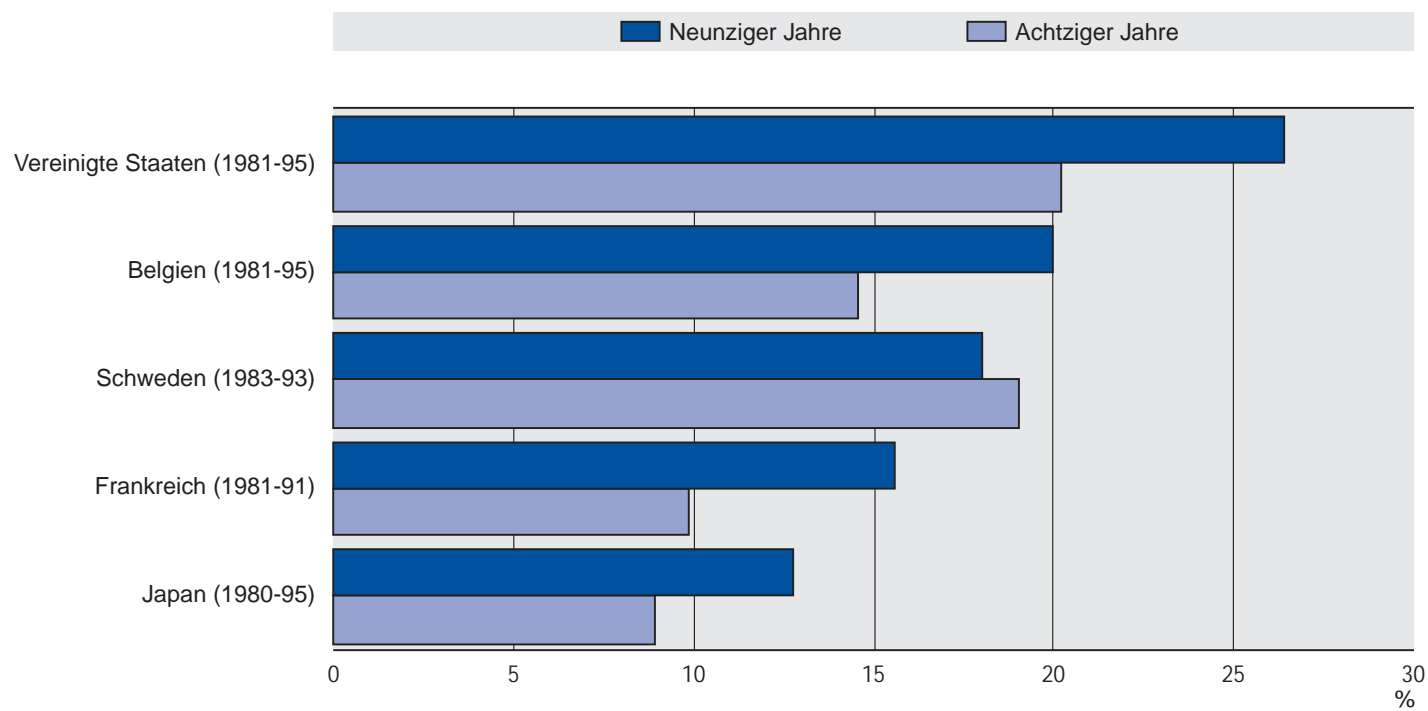

Anmerkung: Erhebungsjahre in Klammern.

Quelle: OECD (1996), auf der Basis von Eurostats Demographics. Bei dem Anteil Alleinerziehender handelt es sich um den prozentualen Anteil von Haushalten mit Kindern und nur einem Erwachsenen. Die Inzidenzrate ist definiert als der prozentuale Anteil der Einelternhaushalte an sämtlichen Familien mit unterhaltsberechtigten Kindern. 
Abbildung B.8 Unterschiede bei den Arbeitsverdiensten zwischen Männern und Frauen, 25- bis 64-jährige Vollzeiterwerbstätige, achtziger und neunziger Jahre

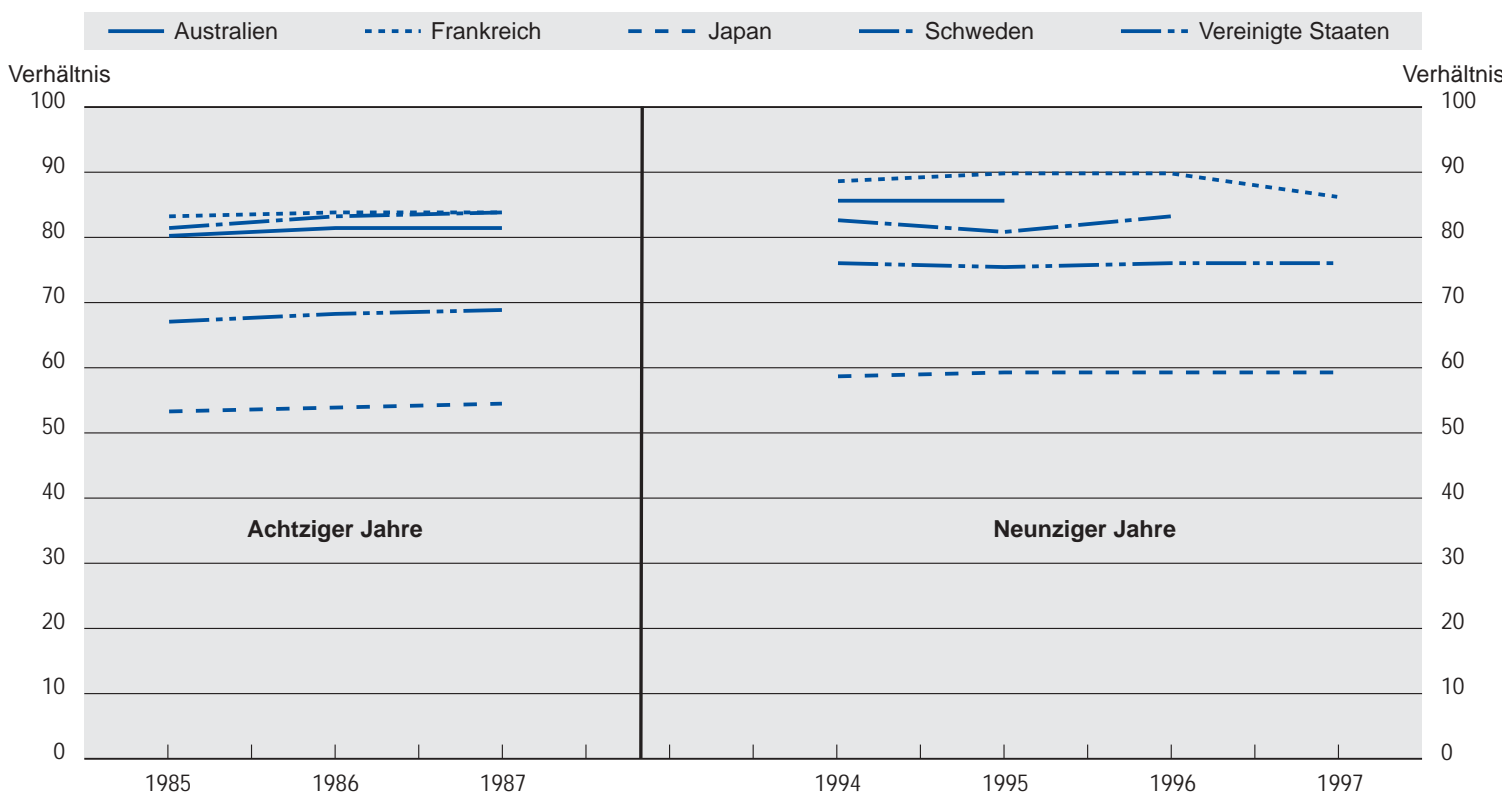

Quelle: OECD Structure of Earnings Database, vgl. Keese und Puymoyen (2001).

Abbildung B.9 Durchschnittliche Erwerbsbeteiligung der 15- bis 64-jährigen Männer und Frauen, in ausgewählten OECD-Ländern, 1960-1999

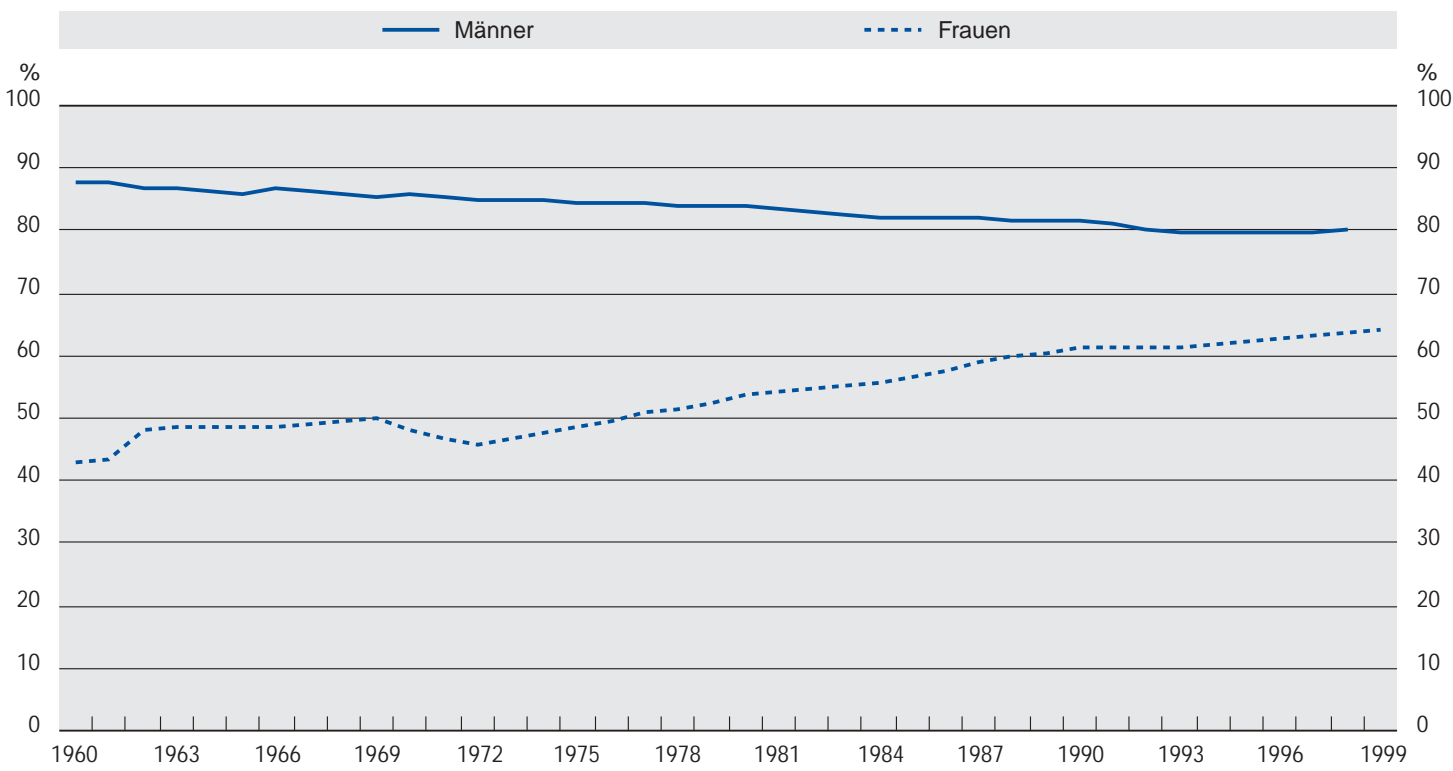

Quelle: OECD, auf der Basis von Arbeitsmarkterhebungen. Der Durchschnitt wurde für folgende Länder errechnet: Australien, Deutschland, Finnland, Frankreich, Italien, Japan, Kanada, Niederlande, Norwegen, Portugal, Schweden, Spanien, Vereinigte Staaten. 
Abbildung B.10 Zugänge von Ausländern in ausgewählten OECD-Ländern, 1999 Je Tausend Einwohner

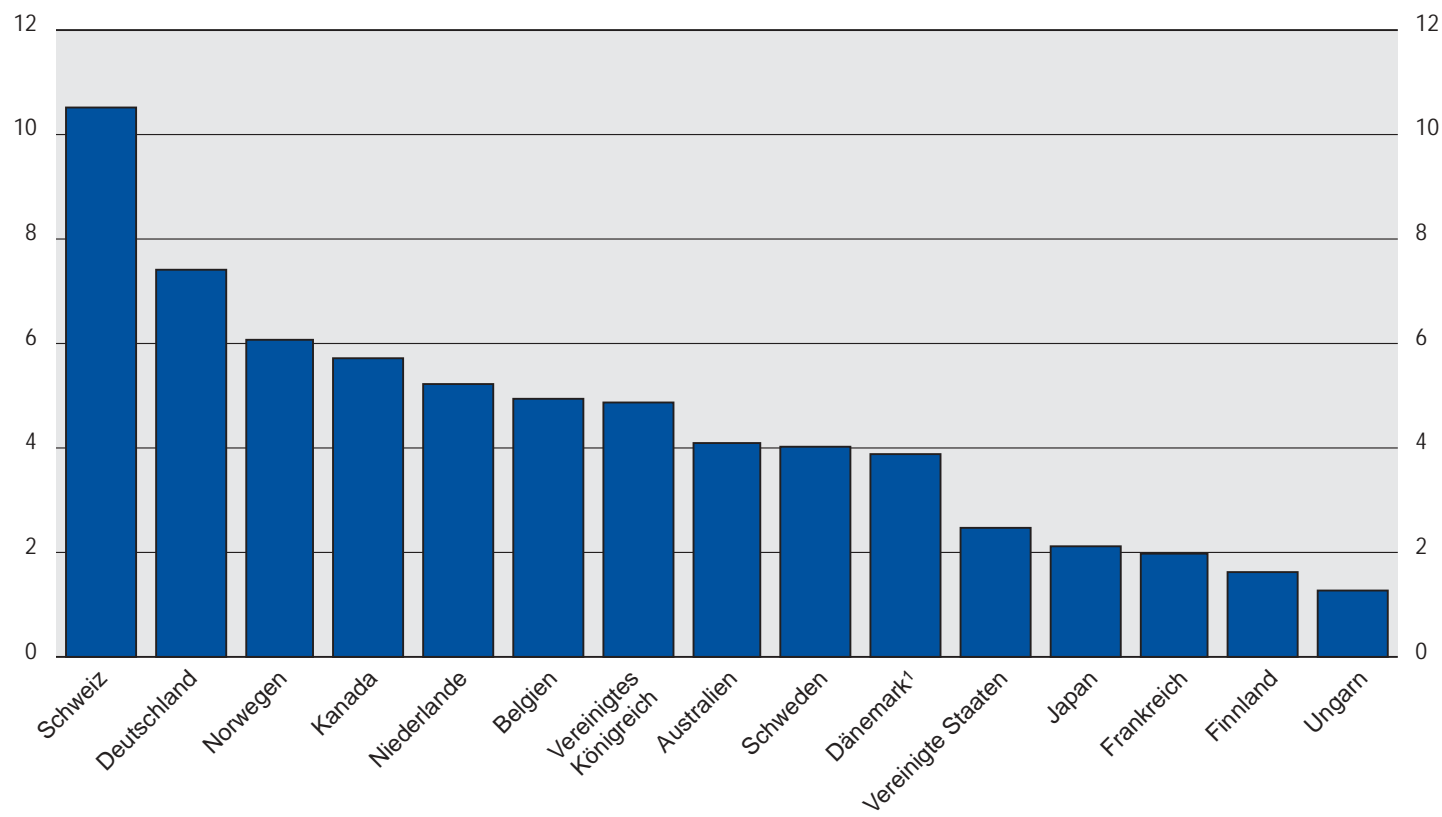

* Die Angaben für Dänemark beziehen sich auf das Jahr 1998.

Quelle: OECD International Migration Database; ISTAT (1999), Jahresbericht.

Abbildung B.11 Zahl der Todesfälle infolge von Drogenmissbrauch in der Europäischen Union Je Million Einwohner, 1986-1997

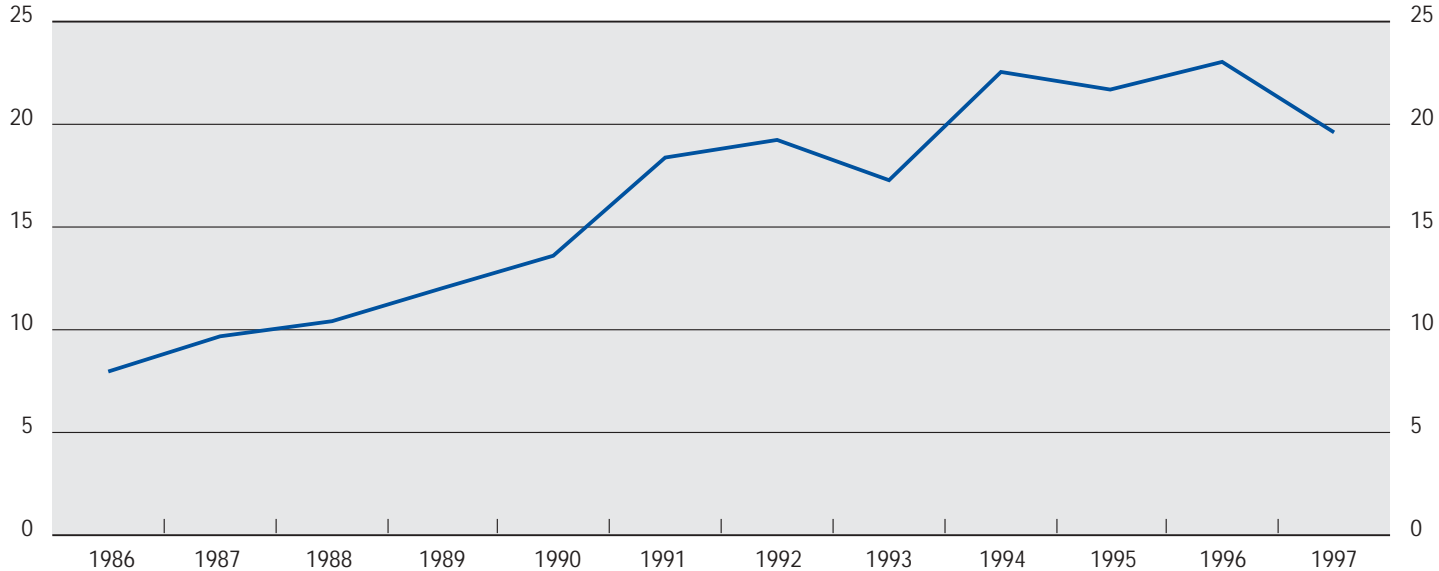

Erfasste Länder: Dänemark, Deutschland, Frankreich, Griechenland, Irland, Italien, Luxemburg, Niederlande, Portugal, Schweden, Spanien und Vereinigtes Königreich.

Quelle: Europäische Ministerkonferenz über Drogen und Drogensucht; UNDCP Redbook (2000), Global Illicit Drug Trends; UN Demographic Projections (Revisionen von 1998). 
Abbildung B.12 Viktimisierungsraten, achtziger und neunziger Jahre

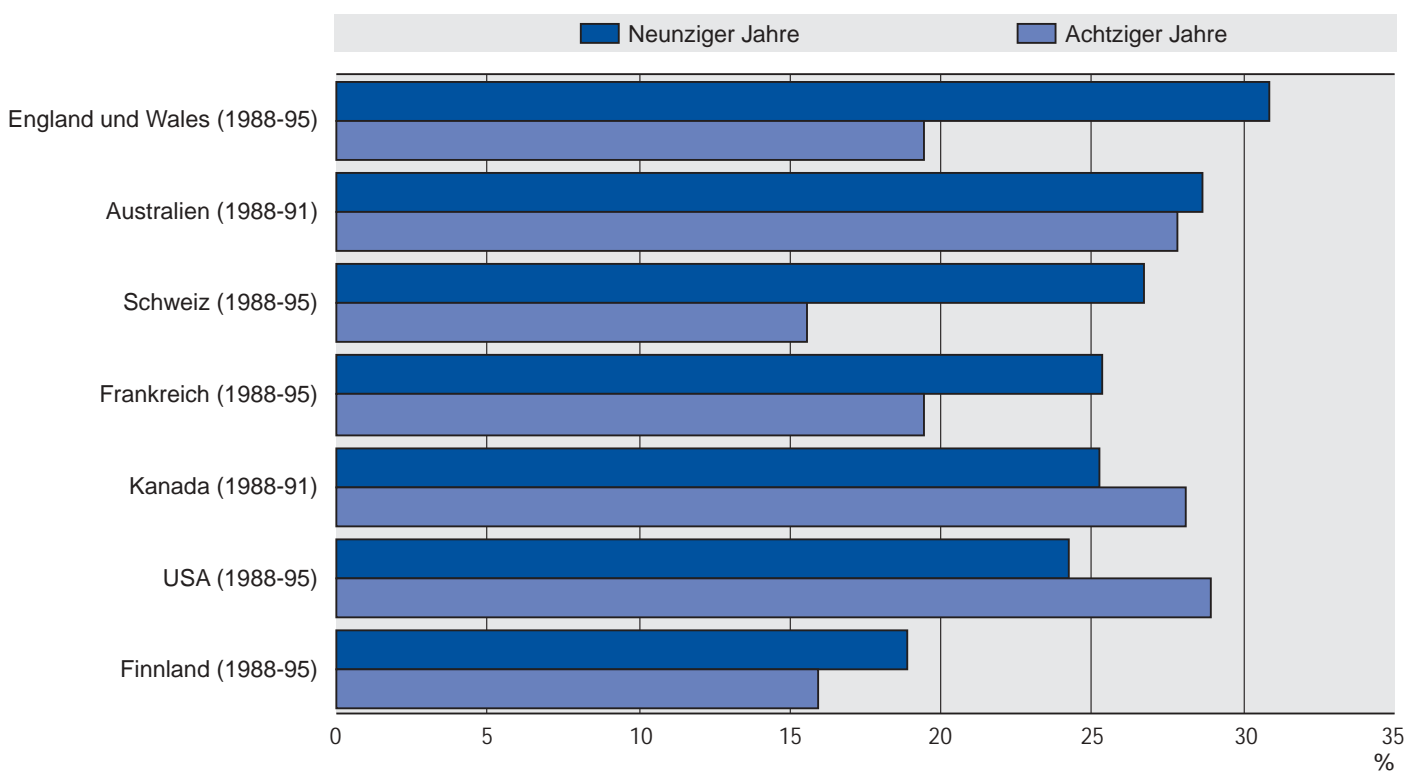

Anmerkung: Prozentualer Anteil der Bevölkerung, der innerhalb eines Jahres Opfer von Straftaten wurde. Erhebungsjahr in Klammern. Quelle: 1996 International Crime Victims Survey.

\section{Abbildung B.13 Durchschnittliche Lebenserwartung bei der Geburt, in ausgewählten OECD-Ländern,} 1960-1998

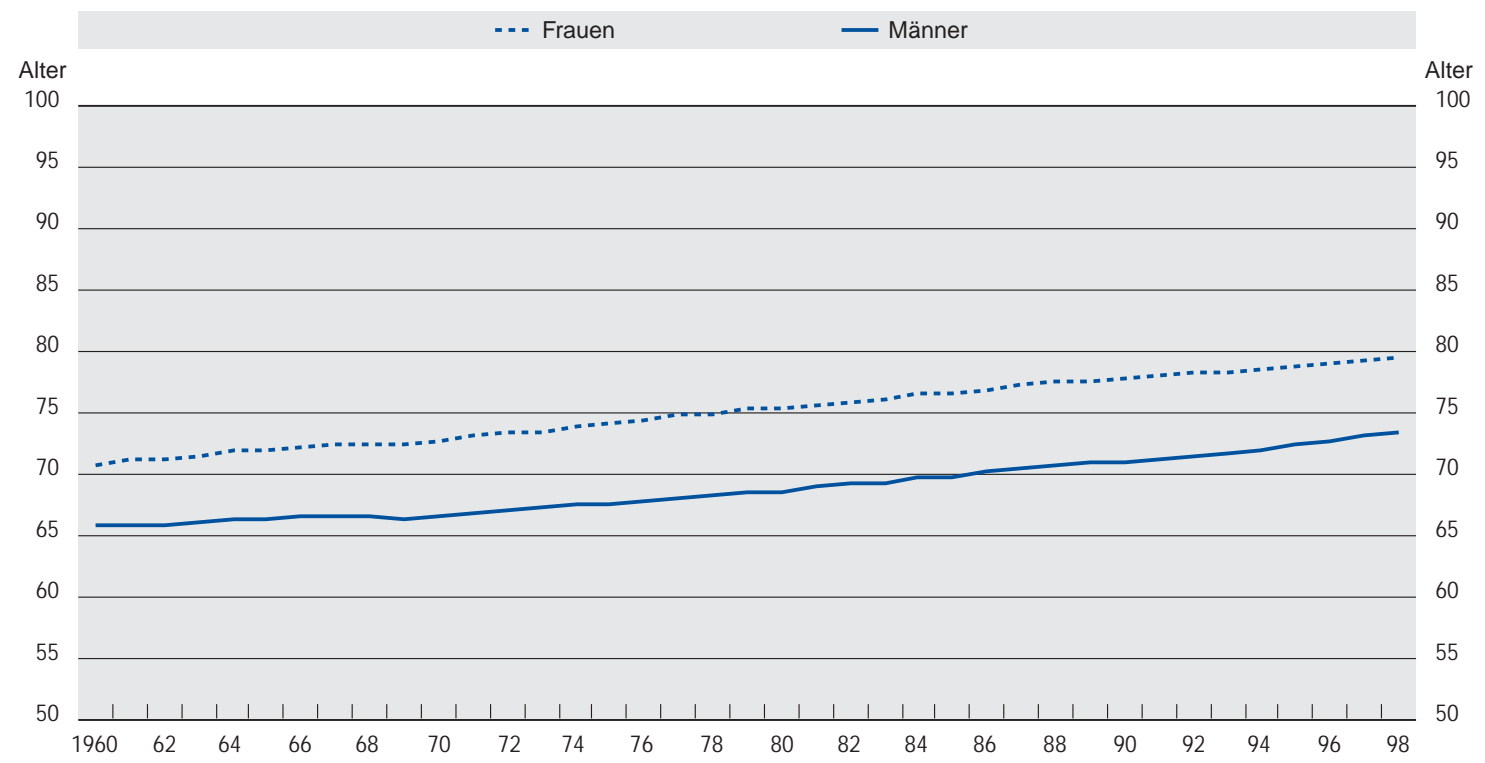

Erfasste Länder: Australien, Belgien, Deutschland, Frankreich, Japan, Mexiko, Neuseeland, Niederlande, Norwegen, Österreich, Polen, Schweden, Tschechische Republik, Türkei, Ungarn, Vereinigte Staaten.

Quelle: OECD (2000), OECD Health Data. 
Abbildung B.14 Jüngste Tendenzen in Bezug auf die Lebenszufriedenheit, 12 ausgewählte Länder der Europäischen Union, 1973, 1983 und 1997

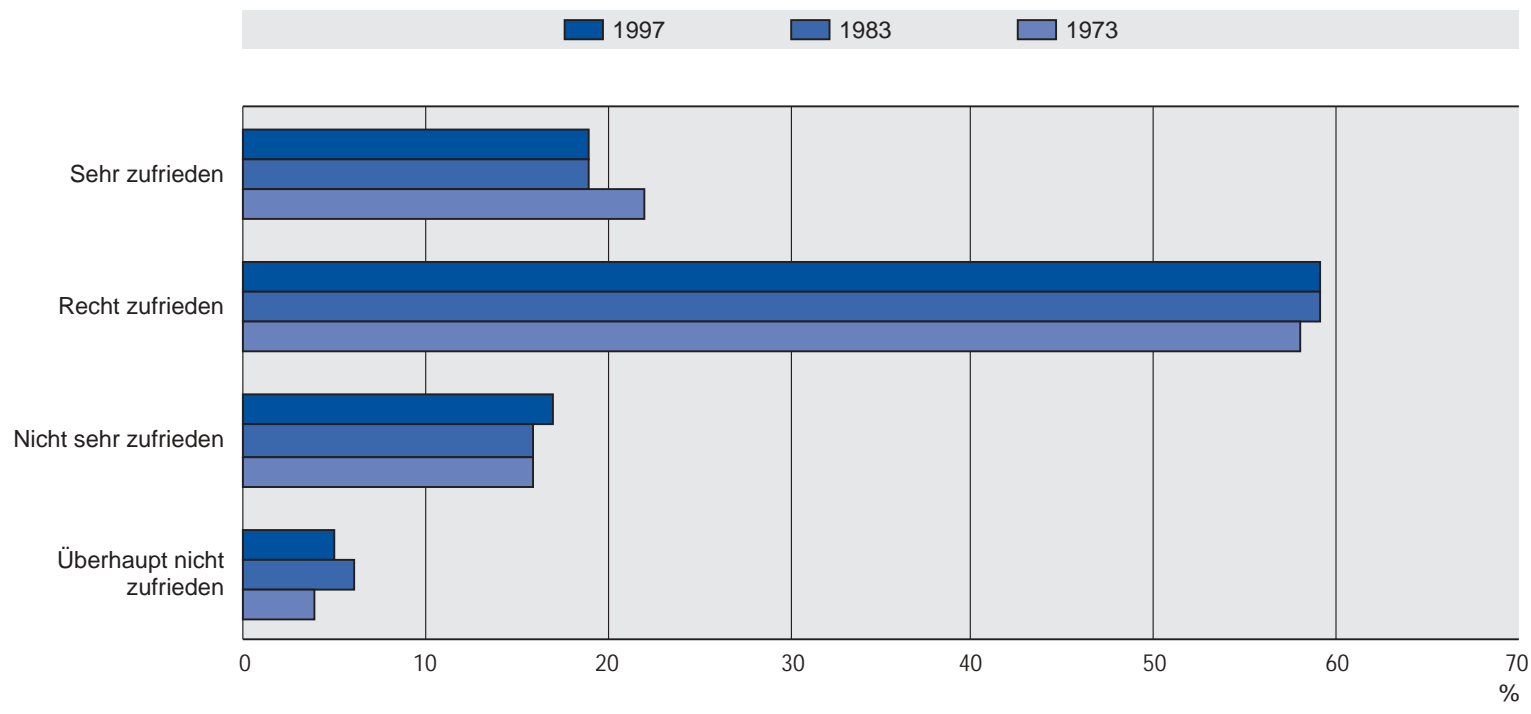

Anmerkung: Die Länder wurden nach ihrer jeweiligen Größe gewichtet: Frankreich, Belgien, Niederlande, Westdeutschland, Italien, Luxemburg, Dänemark, Irland, Vereinigtes Königreich, Griechenland, Spanien, Portugal.

Quelle: Eurobarometers cumulative fille (ICPSR \#9361) für 1973 und 1983 sowie Eurobarometer \#47.1 (ICPSR \#2089) für April 1997, zitiert in Blanchflower und Oswald (2000).

Abbildung B.15 Prozentsatz der jeweiligen Personen, die die Frage nach ihrem subjektiven Glücksempfinden unterschiedlich beantworten, Vereinigte Staaten, 1972-1998

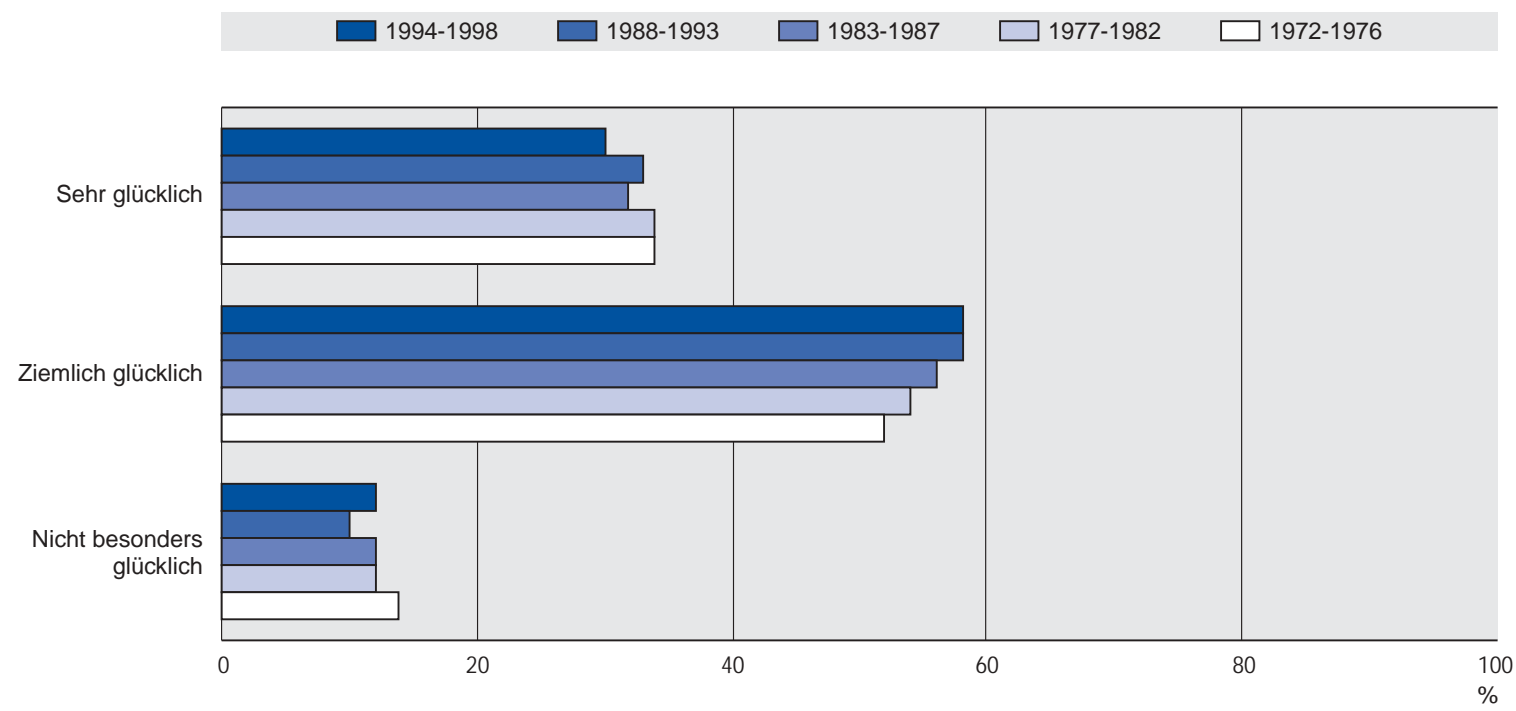

Quelle: US General Social Surveys, zitiert in Blanchflower und Oswald (2000). 
Abbildung B.16 Suizidquote in ausgewählten OECD-Ländern, 1950-1997

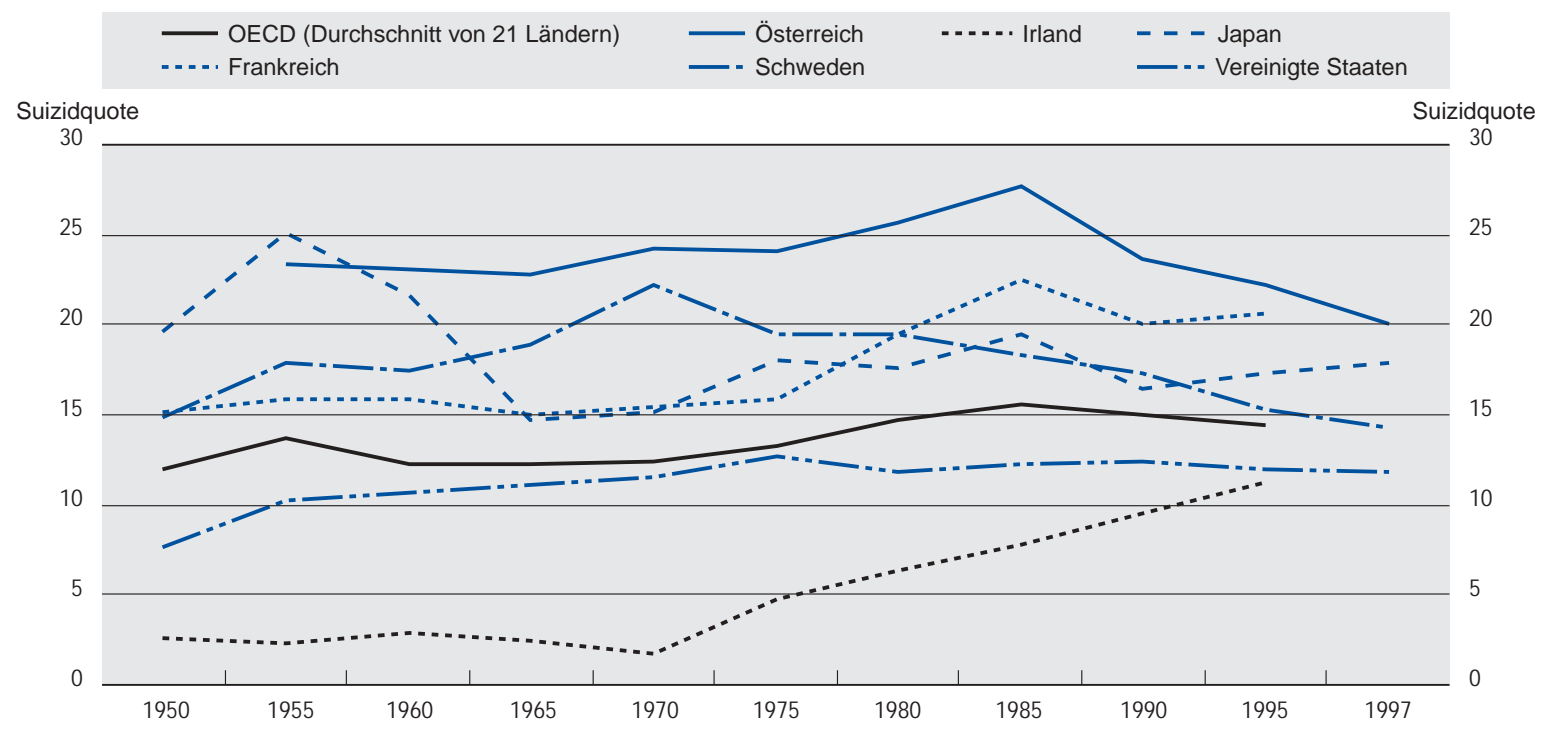

Anmerkung: Der Durchschnitt wurde auf der Basis der folgenden 21 Länder errechnet: Australien, Belgien, Dänemark, Finnland, Frankreich, Griechenland, Irland, Island, Italien, Japan, Kanada, Luxemburg, Neuseeland, Niederlande, Österreich, Polen, Portugal, Schweden, Schweiz, Spanien, Vereinigte Staaten.

Quelle: Weltgesundheitsorganisation.

Abbildung B.17 Treibhausgasemissionen in den OECD-Ländern, gemäß unterschiedlichen Szenarien, 1990-2010

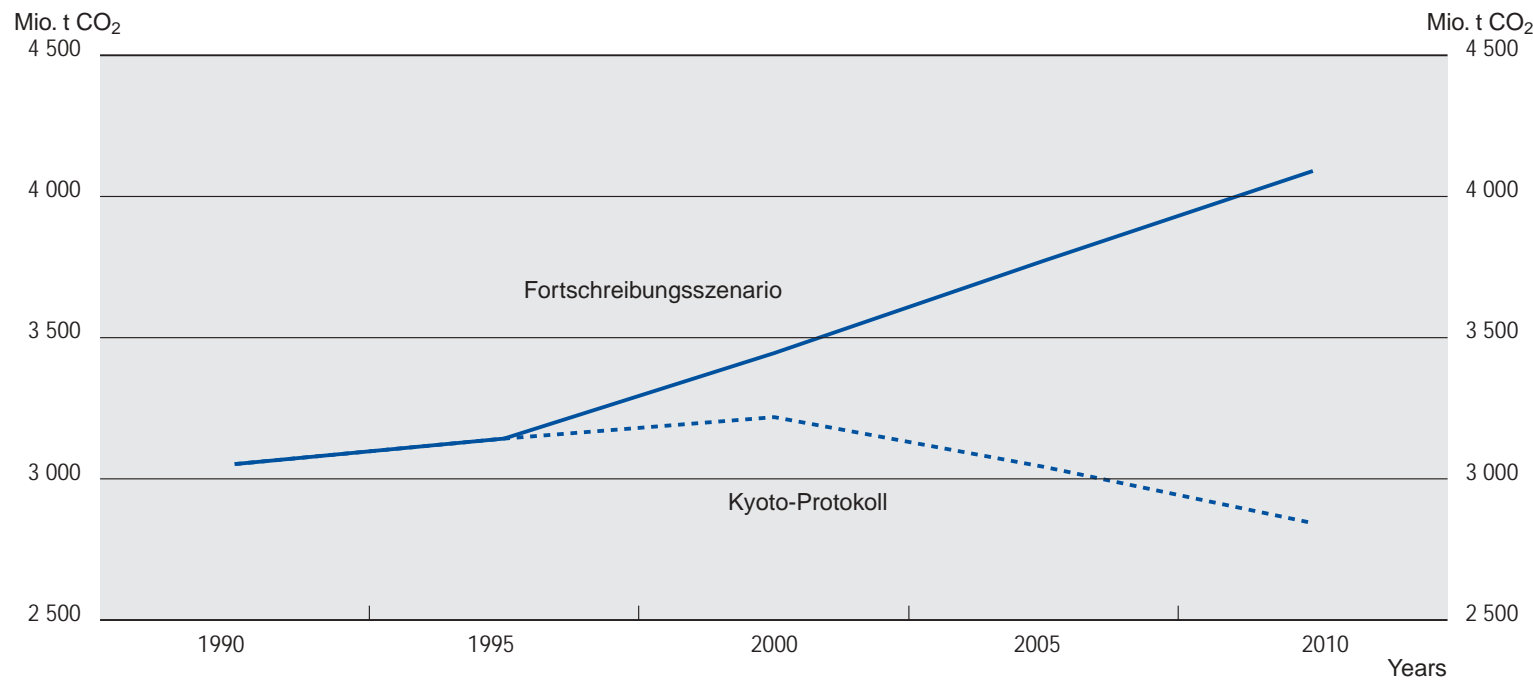

Anmerkung: Die „Kyoto-Protokoll“-Trendkurve beruht auf dem Kyoto-Protokoll von 1997, in dem sich die Industriestaaten für den Zeitraum 2008-2012 zu einer gestaffelten Verringerung der Treibhausgasemissionen gegenüber dem Niveau von 1990 verpflichtet haben. Bei der Trendkurve für das Fortschreibungsszenario wird unterstellt, dass die gegenwärtigen Bedingungen in Zukunft unverändert fortbestehen. Quelle: OECD. Projektionen für die Treibhausgasemissionen gemäß dem OECD-GREEN-Modell. 
Anhang $C$

\section{BESTIMMUNGSFAKTOREN FÜR DAS BILDUNGSNIVEAU: FORSCHUNGSERGEBNISSE}

Dieser Anhang beschreibt die (in Abschnitt 2.4 von Kapitel 2 zusammengefassten) Ergebnisse jüngerer Forschungsarbeiten über die Bestimmungsfaktoren für das Bildungsniveau. Die Belege betreffen überwiegend die Vereinigten Staaten und erstrecken sich lediglich auf eine beschränkte Zahl von Lernergebnissen. Sie legen gleichwohl effektiv die Vermutung nahe, dass das familiäre Milieu und das unmittelbare soziale Umfeld wesentliche Bestimmungsfaktoren für die Lernergebnisse sind und - ebenso wie die Strategien zur Verbesserung der pädagogischen Praktiken - einen wichtigen Ansatzpunkt für die Verbesserung des Bildungsniveaus darstellen. Auch die Aufstockung und bessere Zielorientierung der Finanzmittel, namentlich zwecks Verkleinerung der Klassen, kann im Verein mit einem wirksameren Ressourcenmanagement und effizienteren Lehrpraktiken ebenfalls zu einer Verbesserung der Ergebnisse beitragen. Über die Effekte der materiellen Inputs und der Reform von Lehr- und Lernmethoden hinaus legen die Untersuchungen den Schluss nahe, dass soziale Normen und Netzwerke nach wie vor eine wesentliche Rolle für den Lernprozess in allen Lebensphasen spielen.

Coleman et al. (1966) gelangten in ihrem Bericht über die Ergebnisse einer umfangreichen Erhebung, die Mitte der sechziger Jahre in amerikanischen Schulen durchgeführt worden war, zu dem Schluss, dass es kaum irgendwelche Hinweise auf einen Zusammenhang zwischen Sachaufwand und Klassengröße einerseits sowie Bildungsniveau andererseits gibt (neuere Untersuchungen lassen auf eine gewisse positive Korrelation schließen, fördern aber zugleich Mängel der früheren wie auch der aktuellen Forschungen zu Tage). Coleman stellte fest, dass die Sachinvestitionen in Bildungseinrichtungen die Ergebnisse der Schülerinnen und Schüler nur wenig beeinflussen, vor allem im Vergleich zum familiären Hintergrund. Diese Ergebnisse schwanken allerdings sehr stark je nach der Kategorie der evaluierten Ergebnisse und dem Niveau der wirtschaftlichen und sozialen Entwicklung der untersuchten Bevölkerung. Bei gewissen Lernergebnissen, wie z.B. den mathematischen oder naturwissenschaftlichen Kompetenzen, ist der schulische Einfluss vergleichsweise stärker. Die Qualität der zwischenmenschlichen Beziehungen und vor allem die persönlichen Fähigkeiten weisen zweifellos eine engere Korrelation zum familiären und sozialen Hintergrund als zum schulischen Umfeld auf. Eine weitere Schwierigkeit bei der Unterscheidung zwischen dem familiären und dem schulischen Einfluss besteht in den OECD-Ländern darin, dass es selten möglich ist, diese Effekte im Falle von Jugendlichen zu beobachten, die nicht die Schule besuchen. Demgegenüber verweisen Fuller und Clarke (1994) darauf, dass der Effekt der Schule möglicherweise überbewertet wird, wenn die indirekten Messgrößen für Elternhaus und soziales Umfeld mangelhaft oder nicht sorgfältig genug definiert sind.

Was den Effekt einer Verkleinerung der Klassen betrifft, so sind die Untersuchungsergebnisse gemischt. Hanushek (1998), der nahezu 300 unterschiedliche Schätzungen des Effekts einer Verkleinerung des Klassenumfangs auf die Schulergebnisse untersucht hat, kam zu dem Schluss, dass nichts auf eine positive Korrelation zwischen einer allgemeinen Verringerung der Klassengröße und einer Verbesserung des Durchschnittsniveaus der Schüler hindeutet. Von den 277 Untersuchungen über die Auswirkungen des quantitativen Verhältnisses zwischen Schülern und Lehrern stellte Hanushek lediglich in $15 \%$ der Fälle eine positive und statistisch signifikante Wirkung auf die schulischen Ergebnisse fest, während in 13 Fällen (bzw. bei knapp 5\%) eine signifikante, negative Korrelation bestand. Diesen Beobachtungen zufolge führt eine generelle Verringerung des Klassenumfangs also nicht zwangsläufig zu einer Verbesserung der Schulergebnisse - jedenfalls nicht, so lange sich in Bezug auf die pädagogischen Praktiken, die Motivation der Schülerinnen und Schüler und den Einfluss des familiären Umfelds kein Wandel vollzieht.

Hingegen ließen die Ergebnisse eines in großem Maßstab Mitte der achtziger Jahre im amerikanischen Bundesstaat Tennessee durchgeführten Experiments (STAR-Projekt ${ }^{1}$ ), das auf einer Zufallsauswahl von Schülern basierte, leichte Verbesserungen der Schulergebnisse erkennen, wenn die Kindergarten- bzw. ersten Grundschulklassen verkleinert wurden ${ }^{2}$. In den oberen Klassen scheint der positive Effekt eines verringerten Klassenumfangs, wenn überhaupt, sehr viel weniger signifikant zu sein. Mosteller (1995) kam jedoch auf der Basis von Ergebnissen der STAR- 
Untersuchung sowie anderer Forschungsarbeiten zu dem Schluss, dass eine kleinere Klassengröße Schülerinnen und Schülern aus sozial schwächerem Milieu verhältnismäßig stärker als anderen zugute kommt, was dafür spricht, dass eine gezielte Verringerung der Schülerzahl je Klasse als eine mögliche Maßnahme zur Kompensierung sozialer Ungleichheiten erwogen werden könnte. Auch Nye und Hedges (2000) kamen bei ihrer Auswertung der STAR-Daten zu dem Ergebnis, dass der Klassenumfang sehr wohl eine Rolle spielt. Generell ist jedoch festzustellen, dass die Untersuchungen über den relativen Einfluss des Klassenumfangs bei der Erklärung unterschiedlicher Lernergebnisse auf sämtlichen Bildungsstufen noch vertieft werden müssen. Die Klassengröße (bzw. das zahlenmäßige Verhältnis zwischen Schülern und Lehrern auf dem Sekundarschul- und insbesondere Fach- und Hochschulniveau) variiert in den einzelnen Ländern erheblich je nach Bildungsstufe (von der Primar- bis zur Hochschulstufe), Studienbereich, Schultyp (normale oder Sonderschulen) sowie Standort (städtisches oder ländliches Milieu). Die Möglichkeiten für Veränderungen der Klassengröße und der wahrscheinliche Effekt solcher Veränderungen sind je nach Schultyp und jeweiliger Zielgruppe unterschiedlich.

Die Belege lassen bedeutende Unterschiede bei den Ergebnissen sowohl in als auch zwischen den Schulen erkennen, und das Gleiche gilt für die Effizienz der von den Schulen angewandten pädagogischen Praktiken. Innerhalb der einzelnen Schulen scheinen die unterschiedlichen Lernfortschritte zwischen jeweils zwei aufeinander folgenden Klassen großenteils mit der Qualität der Lehrkräfte zusammenzuhängen (Hanushek, Kain und Rivkin, 1998). Nach Hanushek (1992) können die Differenzen, die bei den Kenntnissen zwischen einem Schüler mit einem guten bzw. mit einem schlechten Lehrer festzustellen sind, innerhalb eines einzigen Schuljahrs dem Lernergebnis von mehr als eineinhalb Schuljahren entsprechen. Bei den einzelnen Lehrern bestehen systematische Unterschiede in Bezug auf die Schnelligkeit, mit der ihre Schülerinnen und Schüler lernen (Murnane, 1975), selbst wenn deren jeweiligen speziellen Merkmalen Rechnung getragen wird. Rivkin, Hanushek und Kain (1998) stellten zudem fest, dass die qualitativen Unterschiede zwischen den Lehrern innerhalb einer Schule stärker ausgeprägt sind als zwischen verschiedenen Schulen eines Distrikts oder einer Region. Desgleichen weisen Folgestudien über den beruflichen Werdegang von Hoch- und Fachhochschulabsolventen darauf hin, dass deren unterschiedliche Arbeitsmarkterfolge mit der Qualität der von ihnen besuchten Schule zusammenhängen (Betts, 1995, und Grogger, 1996).

Andere Untersuchungen befassten sich mit der Wirkung des zwischenschulischen Wettbewerbs auf das Bildungsniveau - ob dieser Wettbewerb nun dadurch zustande kommt, dass die Eltern mehr Auswahlmöglichkeiten unter den verschiedenen Schulen einer Ortschaft haben oder dass Vorkehrungen wie die so genannten Ausbildungsgutscheine existieren, mit denen die Eltern anhand eines öffentlich finanzierten Schecks Bildungsleistungen in der Bildungseinrichtung ihrer Wahl „kaufen“ können. Dieses System der Bildungsgutscheine und seine Effekte bilden in den Vereinigten Staaten nach wie vor Gegenstand hitziger Debatten, und die Analysen der damit erzielten Ergebnisse sind noch nicht abgeschlossen. Hoxby (1994) äußert die Vermutung, dass die Präsenz privater Schulen in einer Region die Effizienz der nahe gelegenen staatlichen Schulen insofern steigert, als diese im Wettbewerb um Schülerinnen und Schüler mit den privaten Einrichtungen zu rivalisieren suchen. Shleifer (1998) zeigt, dass in privater Hand befindliche Schulen nicht nur für größere Wahlmöglichkeiten in Bezug auf Bildungseinrichtungen und für verstärkten interschulischen Wettbewerb sorgen, sondern dass von ihnen auch starke Anreize für Kostenreduzierungen und verbesserte Bildungsergebnisse ausgehen. Eine zentrale Frage lautet jedoch, wie sich die auf der Ebene des Wettbewerbs und der elterlichen Wahlmöglichkeiten eingeführten Änderungen angesichts der ungleichen Chancenverteilung und der unterschiedlichen Ausstattung mit Finanzmitteln und Sozialkapital auf die soziale Gerechtigkeit auswirken werden. Ob solche Innovationen in den Vereinigten Staaten oder anderswo die Qualität des Bildungsniveaus steigern, ohne die soziale Gerechtigkeit zu beeinträchtigen, bleibt abzuwarten. Ferner wird der Aussagewert der Untersuchungen über die verschiedenen Arten vertraglicher Vorkehrungen, und namentlich über die Ergebnisse der privaten im Vergleich zu denen der staatlichen Schulen, einerseits durch das Fehlen geeigneter Kontrollen und andererseits durch eine Reihe von Störfaktoren eingeschränkt ${ }^{3}$.

Einige internationale Untersuchungen geben Hinweise darauf, dass zentralisierte Prüfungen und Kontrollmechanismen für die Einhaltung von Normen und Budgets auf nationaler oder regionaler Ebene zur Verbesserung der Schulleistungen beitragen können ${ }^{4}$ (Wossmann, 2000, und Bishop, 1999). Desgleichen kann das echte Delegieren von Entscheidungsvollmachten an Schulen oder nachgeordnete Gebietskörperschaften, vor allem in Bereichen wie Personalpolitik oder -einstellung sowie in Bezug auf die Beschaffung von Unterrichtsmaterial, den Schulen bei der Verbesserung ihrer Ergebnisse helfen (Wossmann, 2000) ${ }^{5}$.

Die Resultate verschiedener Untersuchungen, die in einer Vielzahl von Ländern durchgeführt wurden (zusammengefasst in Fuller und Clarke, 1994), deuten darauf hin, dass die Verfügbarkeit von Schulbüchern und sonstiger Unterrichtslektüre sowie die Existenz einer Schulbücherei die Schülerleistungen, vor allem auf dem Grundschul- 
niveau, erheblich beeinflussen. Allerdings dürften dabei die Häufigkeit der Inanspruchnahme und die Art des Gebrauchs des Materials bzw. der Einrichtungen mehr ins Gewicht fallen als die bloße Tatsache des Vorhandenseins einer Schulbibliothek.

Es mangelt an empirischen Analysen über den Einfluss, der von den jeweiligen Mitschülern auf die Leistungen der anderen Schüler ausgeht, und die wenigen zu diesem Thema vorliegenden Befunde lassen angesichts der Schwierigkeit, derartige Effekte überhaupt zu ermitteln, keine schlüssigen Folgerungen zu. Hanushek, Kain, Markman und Rivkin (2000) untersuchten die Ergebnisse von Grundschülern (anhand mathematischer und naturwissenschaftlicher Klassenarbeiten) im Bundesstaat Texas und gelangten zu dem Schluss, dass das Leistungsniveau und die ethnische Zusammensetzung der Mitschülergruppe einen unmittelbaren Einfluss auf die von den einzelnen Schülerinnen und Schülern erzielten Ergebnisse hatten ${ }^{6}$. Offenbar profitierten alle Schüler von der Präsenz leistungsstärkerer Mitschüler, wenn der festgestellte Effekt auch nur gering war.

Wie in Kapitel 2 weiter oben dargelegt, spielen vor allem zwei Faktoren eine wesentliche Rolle, nämlich die Zeit, die die Eltern mit ihren Kindern verbringen, sowie die Qualität ihrer Präsenz. Dabei ist das, was die Eltern mit ihren Kindern tun, offenbar wichtiger als deren sozioökonomische Stellung. So wurde z.B. in einer Studie festgestellt, dass die aktive Teilnahme der Eltern am Familienleben stärker ins Gewicht fällt als deren etwaige ehrenamtliche Tätigkeit in der Schule oder ihre Beteiligung an der Schulverwaltung (Ho Sui-Chu und Willms, 1996). Die Autoren ermittelten außerdem, dass die Indikatoren für das elterliche Engagement aussagekräftiger sind als das ursprüngliche soziale Milieu - was die These erhärtet, wonach die Wirkung des sozialen Milieus durch die Stärke des Engagements und die Verhaltensweisen der Eltern erheblich abgeschwächt werden kann. Carlson (1999) beobachtete auf Grund längerer amerikanischer Zeitreihen, dass sich die aktive Teilhabe der biologischen Väter am Leben ihrer Kinder, selbst wenn sie nicht mit ihnen zusammenleben, auf verschiedenste Art und Weise positiv auf das Verhalten der Jugendlichen auswirken kann, so u.a. auf deren Schulbesuchsquote, wobei diese Effekte weitaus größer sind als die der Familienstruktur.

Einige Untersuchungen befassten sich vor allem mit Umfang und Qualität der Netzwerke, die Lehrer, Eltern und lokale Gemeinschaften miteinander verbinden. Colemann nennt als Beispiele für schulnahe Sozialkapitalnetzwerke die Bemühungen und die Zeit, die Eltern dafür aufwenden, enge Beziehungen zu ihren Kindern, den Lehrern und Freunden ihrer Kinder, den Eltern der Kameraden sowie anderen Erwachsenen aufzubauen, die im Leben ihrer Kinder eine wichtige Rolle spielen. Das zwischen den Mitgliedern solcher Netzwerke herrschende gegenseitige Vertrauen schafft ein der Entwicklung der Kinder förderliches Klima und erlaubt deren Überwachung und soziale Beeinflussung. Die Eltern haben dadurch nicht nur die Möglichkeit, sich stärker zu engagieren, sondern sie lernen auch die Freunde ihrer Kinder besser kennen und sind über deren Aktivitäten und schulische Fortschritte genauer informiert. Eine Untersuchung von Coleman und Hoffer über religiöse, private und staatliche Bildungseinrichtungen in den Vereinigten Staaten ergab, dass Konfessionsschulen wesentlich niedrigere Abbrecherquoten als nicht konfessionelle private oder staatliche Schulen aufweisen (Coleman und Hoffer, 1987). Gemäß den Autoren waren die tendenziell besseren Ergebnisse der betreffenden Schulen nicht nur darauf zurückzuführen, dass die dort geltenden religiösen Normen und Vorschriften einen positiven Einfluss auf das Lernen und die pädagogischen Lehrmethoden haben, sondern auch auf die Effekte der „,sozialen Konvergenz“ zwischen Schule und Gemeinschaftsnetzwerken im weiteren Sinne, in die Eltern und Schüler gemeinsam eingebunden sind. Als Lerngemeinschaften weisen Schulen, in denen engere Vertrauensbeziehungen bestehen, bessere Ergebnisse auf, selbst wenn verschiedene andere Faktoren berücksichtigt werden, wie z.B. Ausbildung des Lehrpersonals und individuelle Merkmale der Schülerinnen und Schüler (Bryk, Lee und Holland, 1993, S. 314).

Was die Vorschulerziehung und die außerfamiliäre Betreuung betrifft, so können die Partnerschaften zwischen den jeweiligen Leistungserbringern und den Familien nicht nur die Entwicklung des Kindes, sondern auch die Kompetenzen der Eltern fördern und deren Selbstwertgefühl stärken (Powell, 1989). Die Teilhabe an der effektiven frühkindlichen Entwicklung kann auch stimulierend auf Sozialkapitalnetzwerke wirken, indem Eltern, Betreuer und sonstiges Personal zum Beispiel in die Organisation von Lernaktivitäten, die Durchführung von Programmen und gemeinsame Entscheidungsmechanismen einbezogen werden. Darüber hinaus kann die frühkindliche Erziehung und Betreuung, ebenso wie dies auf der Primar- und Sekundarschulstufe der Fall ist, zur Schaffung von Netzen der gegenseitigen Unterstützung und zur Herstellung enger Verbindungen mit anderen Familien beitragen.

Putnam (2000a) hat Befunde zum Einfluss des Sozialkapitals auf das Bildungsniveau im Fall der Vereinigten Staaten untersucht. Auf der Basis der Ergebnisse des Standard Aptitude Test (SAT) ${ }^{7}$ gelangte er zu dem Schluss, dass eine starke, signifikante Korrelation zwischen den Messgrößen des Sozialkapitals auf der Ebene des jeweiligen Bun- 
desstaats insgesamt und dem individuellen Bildungsniveau besteht (2000a, S. 199-230). Die Ergebnisse waren um zahlreiche potentiell verzerrende Variablen, wie insbesondere Rasse, Einkommensniveau und Einkommensungleichheit, Bildungsstand der erwachsenen Bevölkerung, Armutsquoten, Bildungsausgaben, Gehälter des Lehrpersonals, Klassengröße, Familienstruktur und religiöse Zugehörigkeit sowie Umfang des Privatschulsektors, bereinigt worden. Aber auch wenn den Effekten des Einkommensniveaus und sonstiger Variablen sehr weitgehend Rechnung getragen wurde, ist doch nicht auszuschließen, dass noch andere, nicht erfasste Faktoren die verwendeten Ersatzvariablen des Sozialkapitals und die Bildungsabschlüsse beeinflussen. Die Resultate legen den Schluss nahe, dass:

- ein vergleichsweise höheres Niveau sozialer Interaktion einen offenbar starken positiven Lerneffekt ausübt;

- das Niveau des Vertrauens und der informellen Sozialisierung wichtiger zu sein scheint als der Grad der Mitgliedschaft in Vereinigungen und ähnliche Indikatoren; und

- das Sozialkapital im Vergleich zu herkömmlichen bildungspolitischen Maßnahmen, wie z.B. Verringerung der durchschnittlichen Klassengröße, potentiell offenbar mehr zur Steigerung der Bildungsergebnisse beiträgt.

Nach der Analyse zahlreicher Untersuchungen gelangen Henderson und Berla (1994) zu dem Schluss, dass „... die vorhandenen Belege nunmehr hinreichend überzeugend sind: Wenn Schulen und Familien gemeinsam den Lernprozess unterstützen, werden die Kinder tendenziell nicht nur besser in der Schule abschneiden, sondern auch ihr Leben insgesamt besser meistern ... Wenn sich die Eltern zu Hause um die Schularbeiten ihrer Kinder kümmern, fallen die Resultate in der Schule besser aus. Und wenn sich die Eltern engagieren, bringen es ihre Kinder dort weiter, und die Schulen, die sie besuchen, sind vergleichsweise besser" (Coleman und Hoffer, 1987). Die potentiellen Möglichkeiten, über die derartige Partnerschaften zwischen Schulen, Gemeinschaften und Familien im Hinblick auf die Unterstützung des Lernprozesses verfügen, sind besonders wichtig für Familien aus benachteiligten Regionen und schwächerem sozialen Milieu, die in dreifacher Hinsicht benachteiligt sind, nämlich von ihrem Einkommensniveau, ihren begrenzten Beschäftigungsaussichten und ihrem mangelnden Zugang zu sozialen Netzwerken her.

\section{Anmerkungen}

1. STAR steht für das Experiment Tennessee Student-Teacher Achievement Ratio.

2. Nähere Einzelheiten zu den Ergebnissen des STAR-Projekts finden sich bei Hanushek, 1998. Mit dem STAR-Projekt wurde ein spezielles Experiment durchgeführt, in dessen Rahmen die Kinder auf der Basis einer Zufallsauswahl vom Kindergarten bis zum dritten Grundschuljahr auf Klassen unterschiedlicher Größe aufgeteilt wurden.

3. Wie in anderen Bereichen der Sozialwissenschaften ist es nicht einfach, Untersuchungen auf Grund völliger Zufallsstichproben durchzuführen, da sich die statistischen Kontrollen auf die verwendeten Stichproben beschränken, die aus einem nicht zufälligen Umfeld stammen, so dass sich systematische Fehler bei der Auswahl der Gruppen und Individuen nur schwer vollständig ausschalten lassen.

4. Wobei der Effekt bei der Mathematik allerdings stärker als bei den Naturwissenschaften zu sein scheint. Vgl. Wossmann, 2000.

5. Die Ergebnisse im Hinblick auf die Autonomie der Schulen und die Anwendung zentraler Prüfungen und Standards behalten ihre Gültigkeit sowohl bei einer Mikroanalyse unter Verwendung der TIMSS-Daten (Third International Mathematics and Science Survey) als auch bei Durchführung einer Makroanalyse unter Verwendung von Länderdurchschnitten für die 39 TIMSS-Teilnehmerländer.

6. In dieser Studie wurden unterschiedliche statistische Methoden verwendet, um die Mitschülereffekte von den durch Schule, Gemeinschaft und sonstige Aspekte bedingten Effekten zu trennen.

7. Die von Putnam konstruierte globale Messgröße setzt sich aus folgenden Indikatoren zusammen: $a$ ) Grad des Engagements in Leben und Organisation der Gemeinschaft, $b$ ) öffentliches Engagement (z.B. Ausübung des Wahlrechts), $c$ ) ehrenamtliche Tätigkeiten in der Gemeinschaft, $d$ ) informelle Soziabilität (z.B. Besuche bei Freunden) sowie $e$ ) erfasstes Vertrauensniveau. 
Anhang $D$

AUSWIRKUNGEN DES HUMANKAPITALS AUF DAS WIRTSCHAFTSWACHSTUM:

EINIGE WICHTIGE ERGEBNISSE

() OECD 2004 


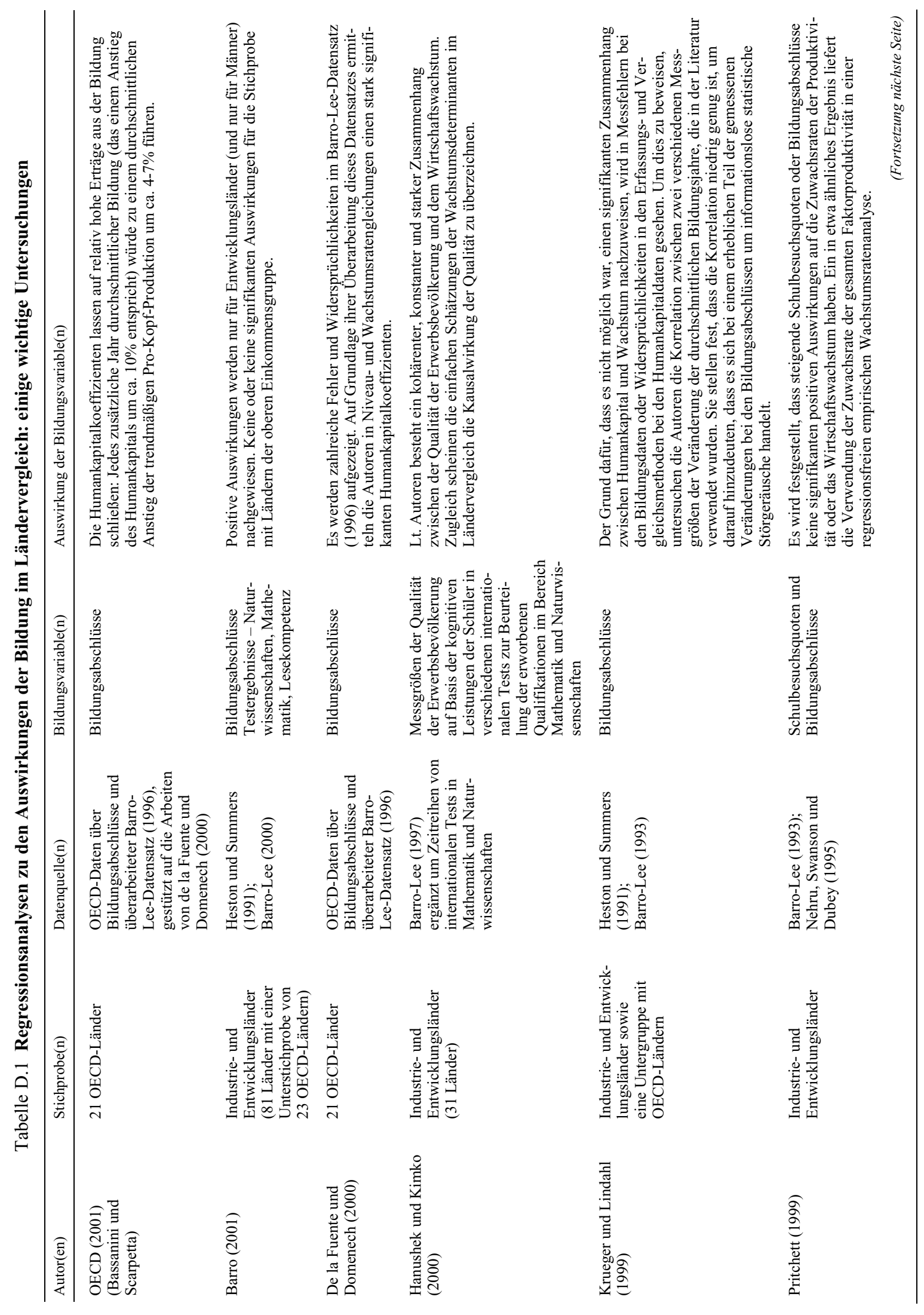

(c) OECD 2004 


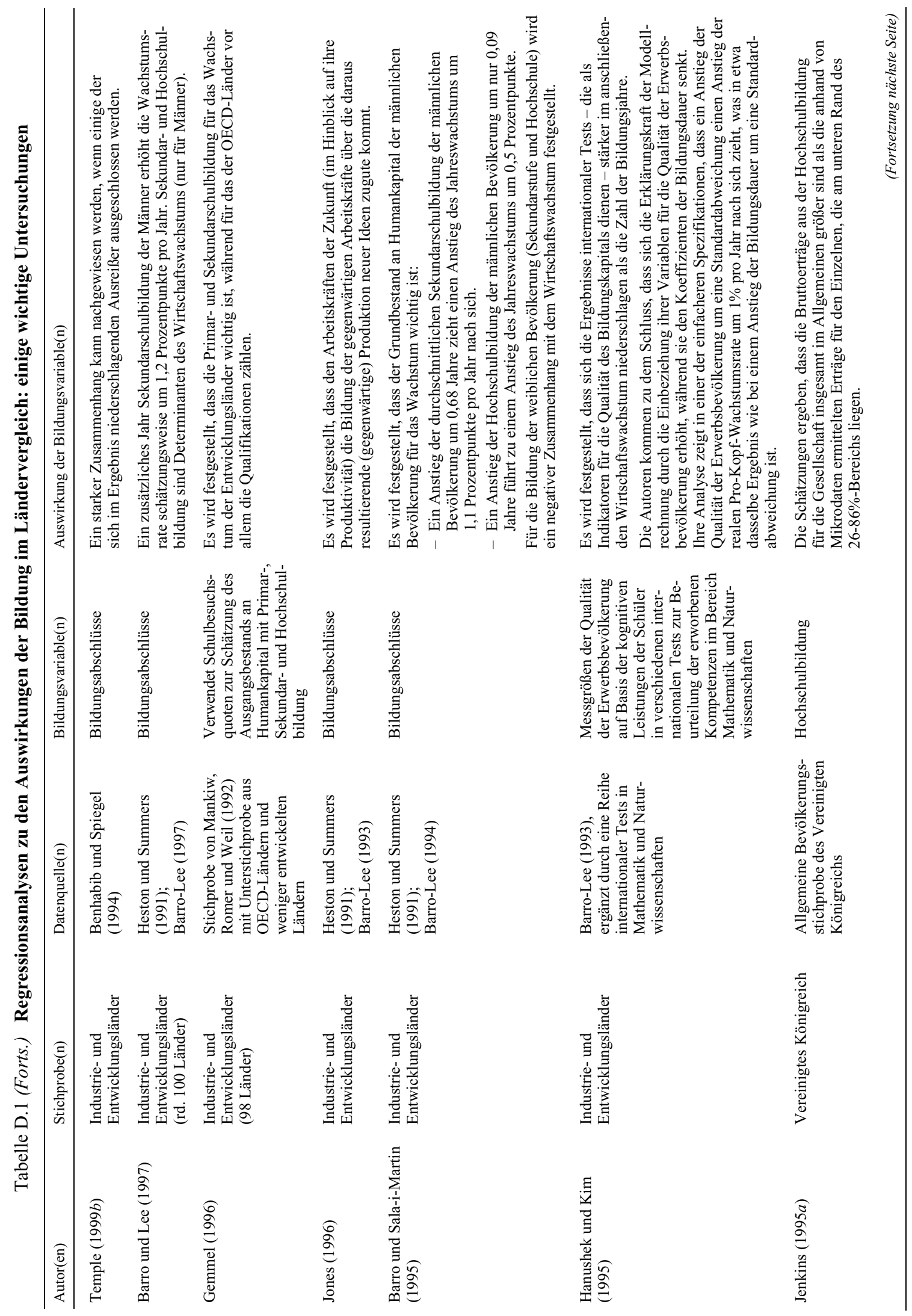




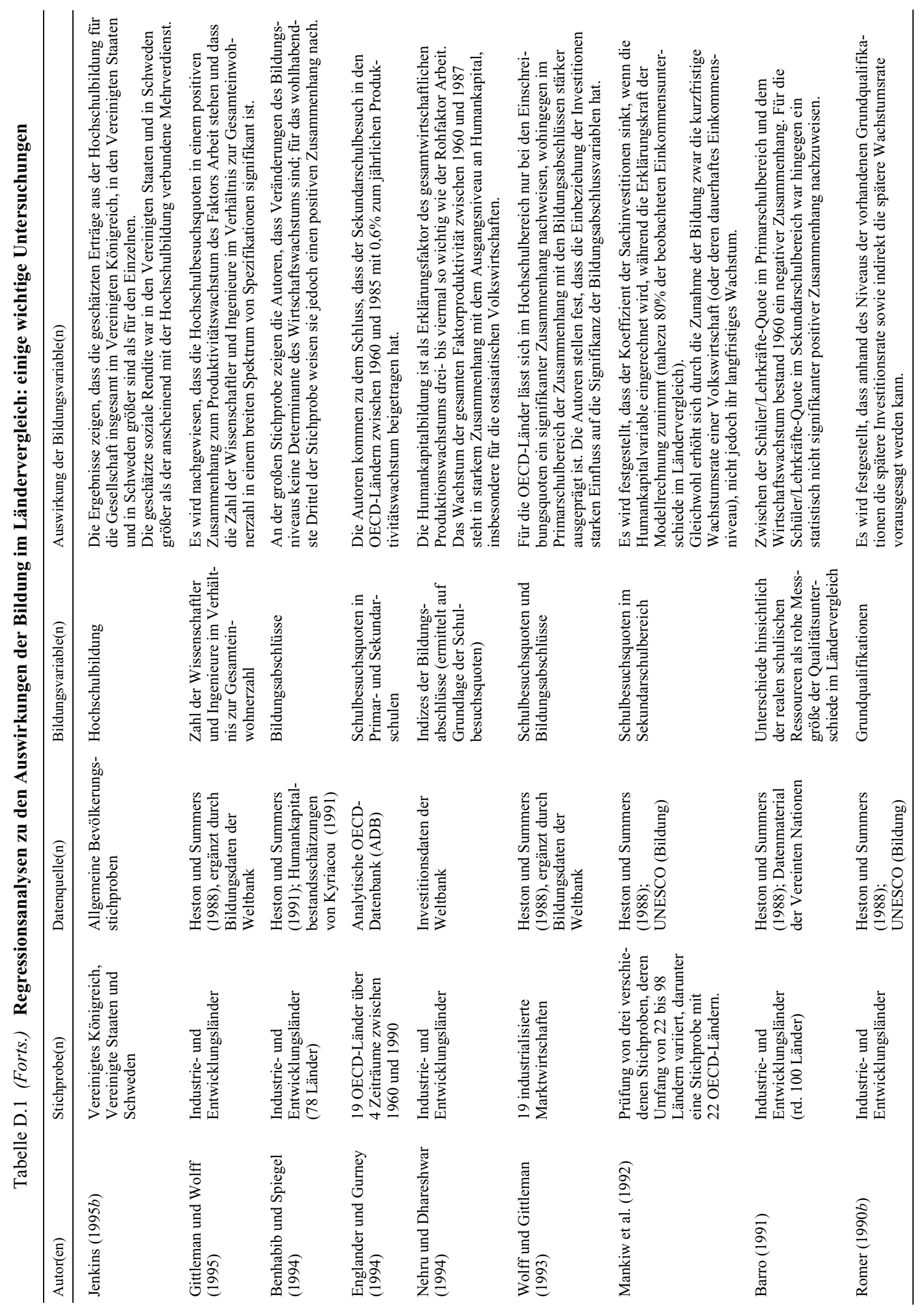

$\overline{\odot \text { OECD } 2004}$ 


\section{NEHMEN VERTRAUEN UND BÜRGERGESELLSCHAFTLICHES ENGAGEMENT IN DEN OECD-LÄNDERN AB?}

Der vorliegende Anhang liefert Informationen zur Situation im Hinblick auf das Vertrauen und das bürgergesellschaftliche Engagement in acht Ländern: den Vereinigten Staaten, dem Vereinigten Königreich, den Niederlanden, Schweden, Australien, Japan, Frankreich und Deutschland.

\section{Vereinigte Staaten}

Putnam (2000a und $b$ ) berichtet von einem deutlichen Rückgang der Gruppenmitgliederzahlen sowie der informellen sozialen Begegnungen in den Vereinigten Staaten. Er verwendet Daten aus verschiedenen Quellen (darunter dem US General Social Survey, dem Roper Social and Political Trends Survey und dem DDN Needham Life Style Survey, die eine Vielzahl von Informationen für mehrere Jahrzehnte liefern). Die Abnahme der Mitgliedsraten formeller Organisationen ging mit einem noch deutlicheren Rückgang der Beteiligungsintensität einher (Zahl der Treffen, Bereitschaft zur Übernahme von Führungsverantwortung usw.). $\mathrm{Zu}$ dieser Abnahme kam es trotz des steigenden Bildungsniveaus, das normalerweise mit einem stärkeren bürgergesellschaftlichen Engagement assoziiert wird. Mitte der siebziger Jahre gaben nahezu zwei Drittel der Amerikaner noch an, dass sie während der vergangenen zwölf Monate an Vereinsversammlungen o.̈. teilgenommen hatten (vgl. Abb. E.1). Ende der neunziger Jahre besuchten nahezu zwei Drittel der Amerikaner niemals solche Versammlungen*. Das politische, bürgergesellschaftliche, religiöse

\section{Abbildung E.1 Schwund der Teilnehmer an Vereinsversammlungen in den Vereinigten Staaten, 1975-1999}

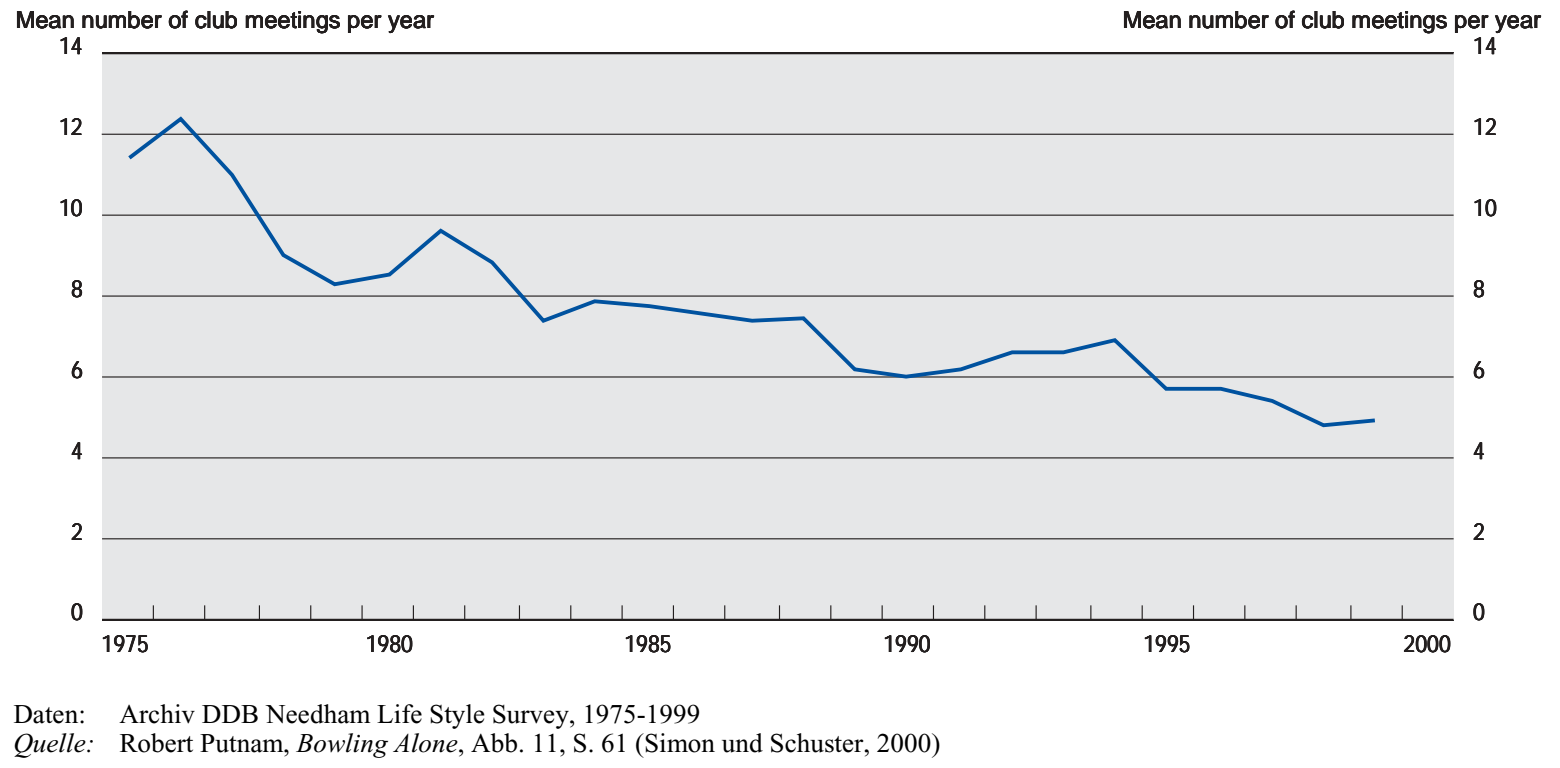

* Zudem sank das Engagement der Amerikaner im Leben von Vereinigungen (ohne Religionsgemeinschaften) gemessen in Stunden pro Monat von 3,7 im Jahr 1965 auf 2,9 im Jahr 1975 und 2,3 in den Jahren 1985 und 1995 (Putnam, 2000a, S. 61-62). 
und berufsständische Engagement hat in den vergangenen 25 Jahren in den Vereinigten Staaten abgenommen, sei es gemessen an der absoluten Mitgliederzahl oder an den „Marktanteilen“ (d.h. den Prozentsätzen einer Zielbevölkerung, die Mitglied einer bestimmten Vereinigung sein kann).

Solche Informationen werfen eine Reihe von Fragen auf. So könnten die festgestellten Veränderungen entweder vorübergehender Natur oder das unweigerliche Resultat sich wandelnder sozialer Normen und Verhaltensmuster sein. Andererseits könnten die beobachteten Trends auch irreführend sein, falls andere, weniger traditionelle Formen sozialer Zusammenschlüsse zugenommen haben, die jedoch nicht in den Langzeitdatenreihen erfasst wurden. Beispielsweise könnten neue soziale Bewegungen wie jene, die sich für Frauen- oder Umweltprobleme, Menschenrechte oder konkrete Einzelfragen einsetzen, an Zulauf gewonnen haben. Die vorliegenden Daten liefern hier ein uneinheitliches Bild, deuten jedoch darauf hin, dass die Teilnahme an solchen Gruppen weniger intensiv und dauerhaft ist als in traditionelleren Arten bürgergesellschaftlicher Vereinigungen. Die Mitgliederzahlen einiger dieser Organisationen steigen und fallen rasch und sind häufig auf „Mailing-Listen“ gestützt. Die Zahl der informellen Selbsthilfegruppen hat zweifellos zugenommen, das Engagement in solchen Gruppen scheint jedoch eher vorübergehender Natur und seltener mit einem Einsatz für öffentliche Interessen verbunden zu sein.

Verschiedene Formen informeller sozialer Vernetzung (z.B. die Häufigkeit von regelmäßigen Essen im Familienkreis, Picknicks, Kartenspielen, Einladungen von Freunden zum Essen, Besuche bei Bekannten und Verwandten, gemeinsamen Restaurantbesuchen usw.) scheinen über einen langen Zeitraum hinweg ebenfalls abgenommen zu haben. Bei der Freizeitgestaltung vollzog sich offenbar eine Entwicklung hin zu individuellen Formen der Beschäftigung oder zu Aktivitäten im engeren Familienkreis, weg von der größeren Gemeinschaft. Die Besucherzahlen von Sportveranstaltungen haben zugenommen (ebenso wie die von Museen, Kinos usw.), die aktive Teilnahme an sportlichen (oder musikalischen) Aktivitäten hat jedoch abgenommen.

Es war ebenfalls eine Abnahme des allgemeinen Vertrauens zwischen den Menschen zu beobachten. Diese Abnahme fällt für die geburtenstarken Jahrgänge (zwischen 1945 und 1965) sowie die „Generation X“ der nach 1965 Geborenen besonders stark aus; diese Altersgruppen haben wesentlich weniger Vertrauen in ihre Mitmenschen als frühere Generationen. Im zeitlichen Verlauf sind die Vertrauensniveaus innerhalb der Alterskohorten unverändert geblieben. Die Wahrscheinlichkeit, dass sie anderen Menschen vertrauen, ist für die Generation der Großeltern mehr als doppelt so groß wie für die der Enkel (50\% gegenüber 20\%). Ihre Wahlbeteiligung ist nahezu doppelt so hoch wie der jüngsten Alterskohorten (80-85\% gegenüber 45-50\%), und sie sind fast doppelt so stark an politischen Fragen interessiert (55\% gegenüber 30-35\%). Die Abnahme des Vertrauens, des politischen Interesses und der Aktionsbereitschaft lässt sich in den USA vor allem zwischen den Generationen beobachten.

Ein weiterer möglicher Indikator für den Schwund des Vertrauens bzw. der Vertrauenswürdigkeit ist die Zunahme der Zahl der Rechtsanwälte ab 1970, die mit einem Phänomen in Zusammenhang steht, das Putnam als „,vorbeugenden Rechtsbeistand“ bezeichnet. Laut Putnam liefert die Tatsache, dass die Zahl der Anwälte bis 1970 trotz des zwischenzeitlich erfolgten tiefgreifenden sozialen und wirtschaftlichen Wandels konstant geblieben ist, einen Hinweis auf spezifische Veränderungen, die sich seit Ende der sechziger Jahre vollzogen haben (vgl. Abb. E.2).

Die Teilnahmequoten an Gemeinde-, Sozial- und Wohltätigkeitsaktivitäten auf freiwilliger Basis sind für Menschen mittleren Alters oder ältere Menschen in der Regel höher als unter den jüngeren Altersgruppen. Altruismus, ehrenamtliches Engagement und Philanthropie stehen in engem Zusammenhang miteinander. Das Opfern von Zeit und Geld hängt stärker vom Engagement in einer Gemeinde als vom Bildungsstand, von der finanziellen Situation, der Gemeindegröße, dem Familienstand oder dem Beruf ab. Trotz des schwindenden bürgergesellschaftlichen Engagements in anderen Bereichen war in den Vereinigten Staaten eine Zunahme des ehrenamtlichen Einsatzes unter den älteren Kohorten zu beobachten (jenen, die Putnam als die „lange Bürgergeneration“ der zwischen 1910 und 1940 Geborenen bezeichnet). Bei der Art des ehrenamtlichen Einsatzes ging die Entwicklung stärker hin zur Hilfe des Einzelnen für den Einzelnen als zum gemeinsamen Engagement auf Gemeindeebene.

\section{Vereinigtes Königreich}

Hall (1999) stellt fest, dass die meisten Formen der Mitgliedschaft in Vereinen und Verbänden seit den fünfziger Jahren zugenommen haben. Dem Vereinigten Königreich und den Vereinigten Staaten ist gemeinsam, dass die bürgergesellschaftliche Kultur dort eine lange Tradition hat, was mit einem hohen Grad an sozialem Vertrauen und politischer und staatsbürgerlicher Beteiligung einhergeht (Almond und Verba, 1963). Anders als im Fall der USA konnte Hall im zeitlichen Verlauf keine Anzeichen eines abnehmenden Engagements feststellen, auch nicht unter den 
Abbildung E.2 Gestiegene Nachfrage nach juristischem und Sicherheitspersonal in den Vereinigten Staaten, 1900-1997

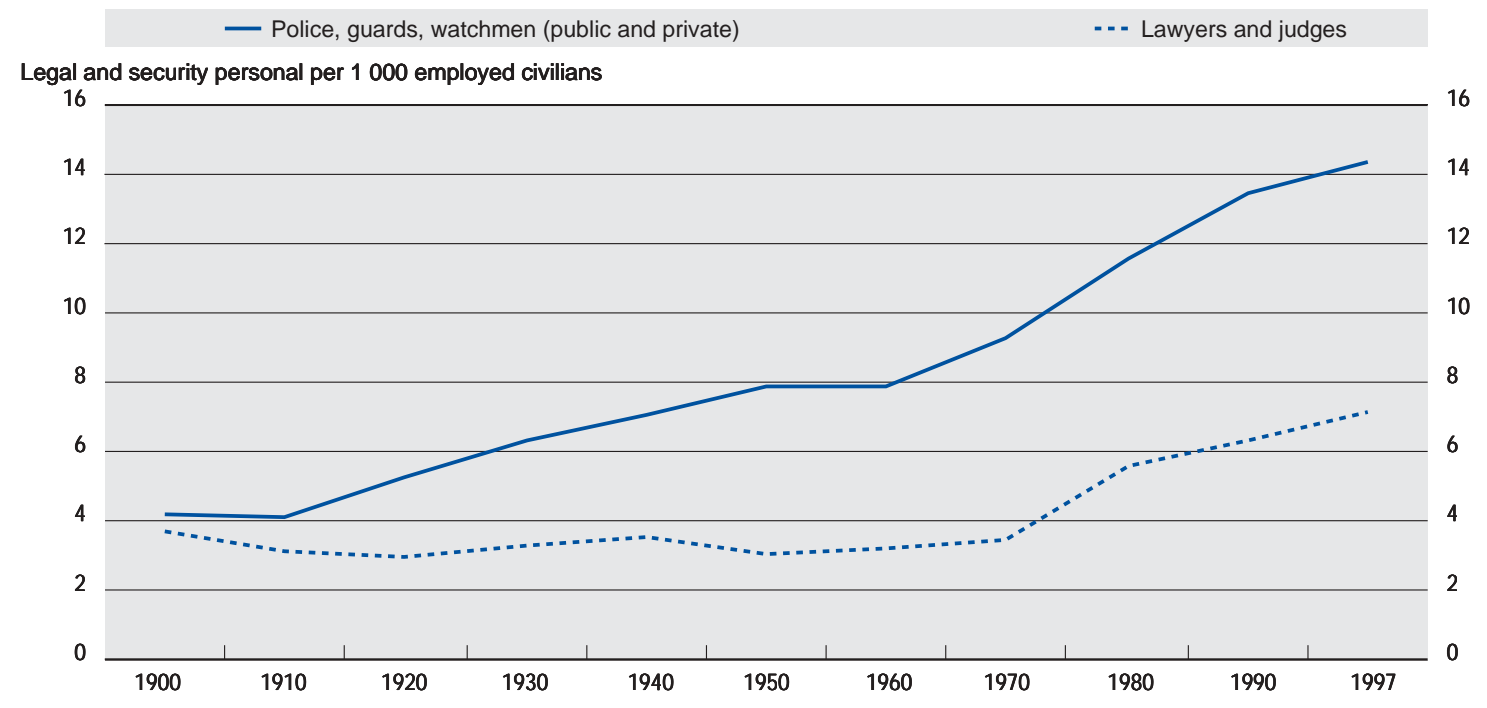

Daten: 1900-1970: Historical Statistics of the United States, Teil I, D589-D592, 144; 1970-1996: Statistical Abstract of the United States sowie Daten des Bureau of Labor Statistics (BLS).

Quelle: Robert Putnam, Bowling Alone, Abb. 42, S. 145 (Simon und Schuster, 2000).

jüngeren Alterskohorten (was die Hypothese widerlegt, dass es zu Veränderungen zwischen den Generationen kommt, die sich in den Daten für die Gesamtbevölkerung noch nicht niedergeschlagen haben). Es war eine gewisse Abnahme der Mitgliederzahlen von traditionellen Frauenvereinigungen oder religiösen Gruppen zu verzeichnen. Allerdings verfügt Hall nicht über Daten zur Entwicklung der Intensität des Engagements oder zu dessen Form im zeitlichen Verlauf. Es ist möglich, dass bei den Mitgliedschaften ein Trend weg von Organisationen, die öffentliche Interessen vertreten, hin zu Selbsthilfegruppen oder auf konkrete Einzelanliegen ausgerichtete Vereinigungen besteht. Denkbar wäre auch, dass an die Stelle persönlicher Zusammenkünfte informellere und weniger dauerhafte Formen des Engagements getreten sind. Die vorliegenden Daten erlauben keine Analyse dieser Eventualitäten.

Hall ist allerdings auf Anzeichen dafür gestoßen, dass sich hinsichtlich der Mitgliedsquoten und der Angaben über das Vertrauensniveau eine wachsende Kluft zwischen verschiedenen sozialen Gruppen auftut, wobei u.a. das unterschiedliche Bildungsniveau ins Gewicht fällt. Es besteht eine positive Korrelation zwischen dem Vertrauen und dem Engagement zum einen und dem Bildungsstand zum anderen; Vertrauen und Engagement sind zudem in der Mittelschicht in der Regel stärker ausgeprägt als in der Arbeiterschicht. Hall stellt auch fest, dass die relative Bedeutung der verschiedenen Gruppen sich parallel zur Abnahme des Anteils der weniger gebildeten Bevölkerungsschichten und den Arbeitern im Vergleich zu den fünfziger Jahren verändert hat. Er vertritt die Ansicht, dass die Bildungsreform und das Wirtschaftswachstum, die mit Veränderungen in der sozialen Klassenstruktur einhergingen, das Vertrauen und das bürgergesellschaftliche Engagement im Vereinigten Königreich gefestigt und damit das Gesamtniveau des Sozialkapitals erhöht haben. Die staatliche Förderung für ehrenamtliche Aktivitäten (soziale Unterstützung, Versorgung älterer Menschen, Gemeindeprojekte usw.) könnte ebenfalls eine wichtige Rolle gespielt haben. Die Beteiligung der Frauen am Gemeindeleben hat zugenommen. Drei diesem Phänomen zugrunde liegende Faktoren konnten identifiziert werden: die gestiegene Teilnahme von Frauen mit Hochschulbildung, die wachsende Beteiligung von erwerbstätigen Frauen sowie allgemeine Veränderungen in der sozialen Stellung der Frau. Der Grad der Teilnahme am Gemeindeleben hat insbesondere in den Bevölkerungskreisen mit Hochschulbildung (bei Frauen gleichermaßen wie bei Männern) stärker zugenommen als in Bevölkerungsgruppen mit anderem Bildungsniveau (obwohl für alle ein Anstieg festzustellen war). Dies könnte u.U. auf einen im Vergleich zur Vergangenheit gestiegenen Grenznutzen der Hochschulbildung für das Sozialkapital hindeuten. 
Die Ergebnisse von Zeitnutzungserhebungen für den Zeitraum von 1961 bis 1984 liefern keine klaren Anhaltspunkte, die auf eine stärkere „Privatisierung“ der Freizeit oder eine Abnahme des Zusammenseins mit anderen hindeuten würden (obwohl die auf Besuche bei Freunden verwendete Zeit abgenommen hat, vor allem unter den männlichen Vollzeitarbeitskräften). Das Vertrauen zwischen den Menschen ist hingegen gesunken. 1959 gaben $56 \%$ aller im Rahmen des UK Civic Culture Survey befragten Personen an, dass sie ihren Mitmenschen im Allgemeinen vertrauen, während dies 1995 nur noch für 31\% der Befragten der Fall war (UK World Values Study). Hall weist darauf hin, dass sich im Laufe der Zeit möglicherweise ein großer Wandel der Wertvorstellungen und Geisteshaltungen vollzogen hat, der sich negativ auf die Qualität des bürgergesellschaftlichen Engagements und das Vertrauen in die Mitmenschen auswirkt. Dies scheint Teil eines allgemeineren Trends hin zu materialistischeren und individualistischeren Werten zu sein, der in Kapitel 1 und 3 beschrieben wurde. Auch Putnam (2000b) stellt deutliche Veränderungen in den Wertvorstellungen und Geisteshaltungen der jungen Menschen im Vergleich zur selben Altersgruppe von vor 20 oder 30 Jahren fest. Der Vertrauensschwund scheint bei jungen Menschen stärker ausgeprägt zu sein als bei älteren. Die Daten für das Vereinigte Königreich sprechen für einen Vertrauensschwund

- zwischen den Menschen (was hier als ,,allgemeines Vertrauen in die Mitmenschen“ bezeichnet wird),

- gegenüber den Institutionen und der staatlichen Verwaltung.

Insgesamt scheint die Situation im Vereinigten Königreich durch eine Zunahme des Vereinslebens sowie informeller Formen sozialen Zusammenseins gekennzeichnet zu sein, die mit einer gleichzeitigen Abnahme des Vertrauens einhergeht. Hinter der gestiegenen Teilnahme an Aktivitäten in Vereinen und Verbänden verbirgt sich eine seit den fünfziger Jahren größer gewordene Kluft zwischen „,eng vernetzten und hoch aktiven Bürgergruppen“ und anderen, deren Beteiligung und bürgergesellschaftliches Engagement sehr beschränkt ist. Die beiden davon am stärksten betroffenen Bevölkerungsgruppen sind die jungen Menschen und die Arbeiterschicht. Dabei fällt auch auf, dass Menschen, die Scheidungen hinter sich haben, arbeitslos waren oder in Großstädte umgezogen sind, bei sonst gleichen Bedingungen seltener dazu neigen, sich Gemeinschaften anzuschließen, und weniger Vertrauen haben.

\section{Niederlande}

Die für die Niederlande vorliegenden Daten lassen auf keinen allgemeinen Schwund des Sozialkapitals schließen, deuten jedoch auf mögliche Veränderungen in der Art des Engagements hin. De Hart und Decker (1999) haben Angaben zu den Mitgliederzahlen verschiedener Organisationsformen und den Beteiligungsquoten an ehrenamtlichen Aktivitäten untersucht. Die Mitgliederzahlen sowie die Intensität des Engagements haben bei den meisten Arten von Organisationen zugenommen (außer bei traditionellen Frauenvereinen und politischen Parteien). Zwischen 1980 und 1985 war keine Veränderung bzw. kein Anstieg der ehrenamtlichen Aktivitäten festzustellen, wenngleich diese Form des Engagements in der Altersgruppe der 18- bis 34-Jährigen abnahm (Dutch Time Budget Survey). Das politische Engagement hat in der Regel zugenommen, und die Wahlbeteiligung bei wichtigen Wahlen ist in etwa unverändert geblieben. Gleichwohl könnte es, wie in anderen europäischen Ländern auch, zu einer Veränderung in der Art des Engagements hin zu weniger intensiven und weniger bindenden Formen gekommen sein. Zu den Interessengruppen, die ein rasches Wachstum verzeichnen, gehören die auf ein konkretes Problem ausgerichteten sozialen Bewegungen, die sich für die Umwelt, die internationale Solidarität oder moralische Fragen einsetzen. Es ist zwar schwierig, das Maß der aktiven Teilnahme an solchen Organisationsformen zu beurteilen, das Engagement könnte hier jedoch eher vorübergehender Natur und weniger auf allgemeine öffentliche Interessen ausgerichtet sein als in anderen Formen bürgergesellschaftlicher Organisationen. Die Erhebungen zur Freizeitgestaltung lassen nicht auf eine Abnahme informeller sozialer Begegnungen oder des informellen Engagements schließen.

\section{Schweden}

Rothstein (1998) hat Daten untersucht, die auf eine Verschlechterung dessen hindeuten, was er als ,organisiertes Sozialkapital“" bezeichnet, nämlich des Vertrauens innerhalb und zwischen den großen Arbeitnehmer- und Arbeitgebervereinigungen, das während einer langen Phase des Konsens und der Beteiligung der Sozialpartner an der wirtschaftlichen und sozialen Planung herrschte. Wie auch in Großbritannien ist ab den achtziger Jahren eine Abnahme des Vertrauens in die politischen Institutionen festzustellen, die mit einem Rückgang des Grads der Zusammenarbeit, der Konsensbereitschaft und des Vertrauens in die Mitmenschen einhergeht. Beim politischen Engagement scheint sich ebenfalls ein Wandel weg von der aktiven Teilnahme hin zum passiven Zuschauen und Interesse zu vollziehen. Die Daten zeigen zwar, dass das politische Interesse zunimmt, es sind jedoch weniger Menschen aktiv in der Politik 
engagiert: Die ehrenamtlichen Parteimitarbeiter sind älter geworden, und die zunehmende Professionalisierung und Medienbegleitung der Politik hat dazu geführt, dass sie immer mehr ins Abseits geraten. Professionell geführte Wahlkampagnen und Medienpräsenz sind zunehmend an die Stelle der Mobilisierung der Bevölkerung, der Diskussionsrunden und der Studienkreise getreten. Wie in anderen europäischen Ländern auch hat die Mitgliedschaft in Organisationen, bei denen ein einzelnes Thema im Mittelpunkt steht, im Vergleich zu der fester Massenorganisationen an Bedeutung gewonnen.

Schweden rangiert zusammen mit anderen skandinavischen Ländern traditionell auf den obersten Plätzen in den internationalen Beurteilungen des organisierten Engagements, des ehrenamtlichen Einsatzes und des (an den Angaben der Befragten gemessenen) Vertrauens zwischen den Menschen. Die Ergebnisse nationaler sowie internationaler Erhebungen (wie der World Values Study) lassen darauf schließen, dass die Vitalität sowohl der formellen als auch der informellen sozialen Organisationen in den letzten Jahrzehnten zugenommen hat. Rothstein ist auf Anzeichen für einen wachsenden Individualismus gestoßen, da sich die jüngeren Menschen von traditionellen, hierarchisch organisierten Formen sozialer Aktivität abgewandt haben. Religiöse Abstinenzbewegungen und Frauenvereinigungen wurden beispielsweise von Freizeit-, Sport-, Kultur- und Umweltvereinigungen verdrängt. Wie im Fall des Vereinigten Königreichs schwankt der Anteil der Organisationsmitglieder zwischen den verschiedenen Bevölkerungsgruppen. Unter jungen Menschen ist er am höchsten. Die Organisationsmitgliedschaft ist im zeitlichen Verlauf in allen Altersgruppen und sozialen Schichten gestiegen, und der Abstand zwischen Frauen und Männern hat sich verringert (wie auch im Vereinigten Königreich). Die Ergebnisse der World Values Study deuten zudem auf eine stärkere Beteiligung in den Jahren 1981 bis 1996 hin. Auch die schwedischen Erhebungen zum Lebensstandard der Jahre 1968, 1981 und 1991 untermauern diese Ergebnisse.

Andere Untersuchungen lassen allerdings auch auf eine Abnahme der Anziehungskraft bestimmter Massenorganisationen schließen. Nach Ansicht von Rothstein könnte dies auf eine gestiegene individuelle Urteilskraft und ein gesunkenes Gemeinschafts- bzw. Gruppenbewusstsein zurückzuführen sein. Wieder andere Studien zeigen, dass der Individualismus und die Autonomie des Einzelnen zugenommen haben. Rothstein meint hierzu, dass dies mit einem stärker „solidarisch“ orientierten Individualismus in Einklang stünde (im Gegensatz zu einem egoistischen Individualismus).

\section{Australien}

Die für Australien vorliegenden Informationen ähneln eher denen der Vereinigten Staaten. Eva Cox (in: Putnam, $2000 b$ ) stellte fest, dass die Mitgliederzahlen vieler seit langem bestehender Freiwilligengruppen in den letzten Jahrzehnten gesunken sind. Beispielsweise sind die Mitgliederzahlen der Gewerkschaften und die Zahl der Kirchenbesuche zwischen den sechziger und den neunziger Jahren beide deutlich zurückgegangen. Der ehrenamtliche Einsatz scheint ebenfalls abgenommen zu haben, während die Daten bezüglich des politischen Engagements kein eindeutiges Bild liefern. Das allgemeine Vertrauen in die Mitmenschen ist ebenso wie das Vertrauen in die politischen Institutionen in den letzten 15 Jahren deutlich gesunken. Es wird mehr ferngesehen, während die informellen sozialen Begegnungen abgenommen haben. Nach Ansicht von Cox könnte der Vertrauensschwund mit zunehmenden Zukunftsängsten und wachsender sozialer Ungleichheit in Zusammenhang stehen. Obwohl der Sport wie in anderen Ländern auch mehr Zulauf zu finden scheint, spiegelt sich in diesem Trend wohl eher eine Zunahme der individuellen Fitnessaktivitäten als des Mannschaftssports wider. Einige soziale Bewegungen, wie z.B. feministische und Umweltgruppen, scheinen an Boden verloren zu haben.

\section{Japan}

Inoguchi (in: Putnam, 2000b) ist in Japan auf Anzeichen für eine Zunahme des Grads an bürgergesellschaftlichem Engagement sowie der Mitgliederzahlen von Nichtregierungsorganisationen gestoßen. Bei den Mitgliederzahlen von Nachbarschaftsvereinigungen sind seit Mitte der achtziger Jahre keine nennenswerten Veränderungen zu beobachten. Die Teilnahme an ehrenamtlichen Betreuungsstrukturen für Kinder, ältere Menschen und sozial Benachteiligte hat deutlich zugenommen.

Der „Radius“ des sozialen Vertrauens ist in Japan möglicherweise enger als in Nordeuropa oder den Vereinigten Staaten. Inoguchi vertritt die Meinung, dass das Vertrauen und die Zusammenarbeit im kleinen Kreis der Familie, der engeren Freunde, der Kollegen oder Geschäftspartner größer ist. Fukuyama (1995) ist ebenfalls der Ansicht, dass kennzeichnend für die Natur des Vertrauens in der japanischen Gesellschaft das Entstehen großer Unternehmen aus kleinen Familienfirmen auf Grund einer reichen und komplexen Zivilgesellschaft ist (S. 130). Die „schlanke“ Produk- 
tion wird in Japan als Modell des Vertrauensarbeitsplatzes (high trust workplace) dargestellt, wo die Rolle des einzelnen Arbeiters darin besteht, seine Urteilskraft einzubringen, damit die gesamte Produktionskette funktioniert (S. 258-259). Es scheint Anzeichen für eine allmähliche Zunahme des allgemeinen Vertrauens in die Mitmenschen zu geben, was darauf hindeutet, dass Japan allmählich die in anderen Ländern vorherrschenden Verhaltensmuster übernimmt. Es liegen nur wenige Daten über das Ausmaß der informellen sozialen Begegnungen vor.

\section{Frankreich}

Die von Worms (in: Putnam, 2000b) untersuchten Daten deuten nicht auf einen Rückgang der Beteiligung am Leben von Vereinen und Verbänden hin. Zwar sind die Mitgliederzahlen einiger großer sozialer und politischer Organisationen gesunken, insbesondere der Gewerkschaften, der politischen Parteien und der Kirche, die Mitgliederzahlen anderer Arten von Vereinigungen sind jedoch unverändert geblieben. Sozialpolitik und Sozialfürsorge könnten sozialen Dienstleistungsorganisationen ohne Erwerbscharakter Auftrieb gegeben haben. In den letzten Jahrzehnten fallen zwei Trends ins Auge: erstens eine Zunahme der Organisationen, die sich für die Interessen einzelner Sektoren oder Gruppen einsetzten, und daran anschließend ein Anstieg der Mitgliedszahlen von Organisationen, die allgemeinere Interessen vertreten; zweitens eine Zunahme der Zahl der Vereinigungen, die der persönlichen Entfaltung durch Kultur- und Freizeitaktivitäten dienen. Diese Veränderungen scheinen z.T. mit dem Bildungsniveau in Zusammenhang zu stehen.

Wie in anderen Ländern auch scheint sich bei der bürgergesellschaftlichen Beteiligung eine Entwicklung hin zu informelleren und vorübergehenderen Formen der Mitgliedschaft vollzogen zu haben. Worms ist der Meinung, dass der Wandel in der Art des Engagements auf ein „fehlendes Glied“ zwischen dem privaten Sozialleben und dem allgemeineren öffentlichen Interesse hindeutet, insbesondere was die Beziehungen zu den öffentlichen Institutionen anbelangt. Gleichwohl ist der Unterschied beim Grad des bürgergesellschaftlichen Engagements zwischen Frankreich und anderen Ländern nach wie vor erheblich. Was die Zahl der Mitglieder von Vereinigungen, der ehrenamtlich Tätigen und der Spender anbelangt, liegt Frankreich nicht nur hinter den Vereinigten Staaten und den skandinavischen Ländern zurück, sondern auch hinter Deutschland, Belgien, dem Vereinigten Königreich und Irland. Worms nennt als mögliche Ursache dafür den historischen Einfluss von Kirche und Staat, die einander ihre Rolle in der Zivilgesellschaft streitig machten und somit wenig Raum für Initiativen seitens der Bürger ließen.

\section{Deutschland}

Die Beteiligung an formellen Organisationen sowie informellen sozialen Begegnungen, die sich 1945 auf sehr niedrigem Niveau befand, hat in Deutschland zugenommen (Offe und Fuchs, 1998). Besonders augenfällig ist dies für die jüngeren Generationen. Es gibt indes zwei große Ausnahmen: Erstens sind die Mitgliederzahlen der Gewerkschaften, der politischen Parteien und der Kirchen gesunken und zweitens scheint das Engagement der jungen Deutschen in politischen und sozialen Organisationen während der neunziger Jahre nachgelassen zu haben. Wie in Schweden auch gibt es Hinweise auf einen Trend weg von formellen Mitgliederorganisationen hin zu vorübergehenderen und individuelleren Arten des Engagements. Die Verteilungsmuster des sozialen Engagements für die verschiedenen Bevölkerungsgruppen sind ähnlich gelagert wie in den Vereinigten Staaten: Das Engagement in Vereinen und Verbänden (insbesondere formeller Art) ist in den gebildeten und wohlhabenderen Bevölkerungsschichten stärker ausgeprägt, ebenso wie unter der Arbeiterschaft, bei Menschen mittleren Alters, in Kleinstädten und unter der männlichen Bevölkerung (die sich vor allem in „öffentlicheren“ Aktivitäten engagiert, obwohl sich auch hier die Lücke zwischen den Geschlechtern allmählich schließt). 


\section{LITERATURVERZEICHNIS}

ABRAMOVITZ, M. und P. DAVID (1996),

"Convergence and Deferred Catch-up: Productivity Leadership and the Waning of American Exceptionalism", in: R. Landau, T. Taylor und G. Wright (Hrsg.), The Mosaic of Economic Growth, Stanford University Press, Stanford, CA.

ABRAMS, P. und M. BULMER (1986),

Neighbours, Cambridge University Press, Cambridge, Vereinigtes Königreich.

ACEMOGLU, D. (1996),

“A Microfoundation for Social Increasing Returns in Human Capital Accumulation”, Quarterly Journal of Economics, Vol. 111, S. 779-804.

ADLER, P. und S. KWON (2000),

"Social Capital: The Good, the Bad, and the Ugly", University of Southern California.

AGHION, P. und P. HOWITT (1998),

Endogenous Growth Theory, MIT Press, Cambridge, MA.

ALESINA, A. und D. RODRIK (1992),

"Income Distribution and Economic Growth: A Simple Theory and Empirical Evidence", in: A. Cukierman, Z. Hercowitz und L. Leiderman (Hrsg.), The Political Economy of Business Cycles and Growth, MIT Press, Cambridge, MA.

ALMOND, G. und S. VERBA (1963),

The Civic Culture: Political Attitudes and Democracy in Five Nations, Princeton University Press, Princeton.

AMATO, P. (1998),

"More than Money? Men's Contributions to their Children's Lives", in: A. Booth und A. Creuter (Hrsg.), Men in Families: When do they get involved? What difference does it make?, Lawrence Erlbaum, New Jersey, Kapitel 13.

ANGRIST, J. und A.B. KRUEGER (1991a),

"Does Compulsory School Attendance Affect Schooling and Earnings?", Quarterly Journal of Economics, Vol. 106, S. 979-1014.

ANGRIST, J. und A.B. KRUEGER (1991b),

"Estimating the Payoff to Schooling Using the Vietnam-Era Lottery", Princeton University Industrial Relations Section, Working Paper, No. 290, August.

ARROW, K.J. (1972),

"Gifts and Exchanges," Philosophy and Public Affairs, No. 1, Sommer.

ASHENFELTER, O. und A.B. KRUEGER (1994),

"Estimates of the Economic Return to Schooling from a New Sample of Twins", American Economic Review, Vol. 84, No. 5, Dezember, S. 1157-1173.

AXELROD, R. (1984),

The Evolution of Cooperation, Penguin, New York.

BAKER, B. und D. BENJAMIN (1994),

"The Performance of Immigrants in the Canadian Labor Market", Journal of Labour Economics, Vol. 12, No. 3.

BARBIERI, P., H. RUSSELL und S. PAUGAM (1999),

"Social Capital and Exits from Unemployment", unveröffentlichtes Papier.

BARRO, R.J. (1991),

"Economic Growth in a Cross-section of Countries", Quarterly Journal of Economics, CVI, Mai. 
BARRO, R.J. (2001),

"Education and Economic Growth", in: J.F. Helliwell (Hrsg.), The Contribution of Human and Social Capital to Sustained Economic Growth and Well-being: International Symposium Report, Human Resources Development Canada und OECD.

BARRO, R.J. und J.W. LEE (1993),

"International Comparisons of Educational Attainment", NBER, Working Paper, No. 4349.

BARRO, R.J. und J.W. LEE (1994),

"Data Set for a Panel of 138 Countries", überarbeitete Auflage, Januar.

BARRO, R.J. und J.W. LEE (1996),

"International Measures of Schooling Years and Schooling Quality", American Economic Review, Papers and Proceedings 86, No. 2, S. 218-223.

BARRO, R.J. und J.W. LEE (1997),

"Schooling Quality in a Cross-section of Countries", NBER, Working Paper, No. 6198.

BARRO, R.J. und J.W. LEE (2000),

"International Data on Educational Attainment: Updates and Implications", Center for International Development (CID), Working Paper, No. 42, Harvard Universität, April.

BARRO, R.J. und X. SALA-I-MARTIN (1995),

Economic Growth, McGraw-Hill, New York.

BASSANINI, A. und S. SCARPETTA (2001),

"Links between Policy and Growth: Evidence from OECD Countries", OECD, Economics Department Working Papers.

BECKER, G. (1993),

"Human Capital: A theoretical and Empirical Analysis, with Special Reference to Education", The University of Chicago Press, Chicago, 3. Auflage.

BEHRMAN, J.R. und N. STACEY (Hrsg.) (1997),

The Social Benefits of Education, The University of Michigan Press, Ann Arbor.

BENHABIB, J. und M. SPIEGEL (1994),

"The Role of Human Capital in Economic Development: Evidence from Aggregate Cross-Country Data", Journal of Monetary Economics, Vol. 43, S. 143-174.

BETTS, J. (1995),

"Does School Quality Matter? Evidence from the National Longitudinal Survey of Youth", Review of Economics and Statistics, Mai, No. 77(2), S. 231-250.

BETTS, J. und J. ROEMER (1998),

"Equalizing Opportunity through Educational Finance Reform”, Department of Economics, University of California, San Diego.

BIBLARZ, T., A. RAFTERY und A. BUCUR (1997), "Family Structure and Social Mobility", Social Forces, Vol. 75(4), S. 1319-1339.

BISHOP J. (1999),

"Are National Exit Examinations Important for Educational Efficiency?", Swedish Economic Policy Review, Vol. 6(2), S. 349-398.

BLANCHFLOWER, D.G. und A.J. OSWALD (2000),

"Well-being over Time in Britain and the USA", NBER, Working Paper, No. 7487, Cambridge, MA (http://www.nber. org/ papers/w7487).

BLOSSFELD, H.P. und Y. SHAVIT (1993), Persistent Inequality: Changing Educational Attainment in Thirteen Countries, Westview Press Inc, Colorado.

BOOTHBY, D. (1999),

"Literacy Skills, the Knowledge Content of Occupations and Occupational Mismatch", Applied Research Branch Research Papers, Human Resources Development Canada, August.

BORJAS, G.J. (1998),

“The Economic Progress of Immigrants”, NBER, Working Paper, No. 6506.

(C) OECD 2004 
BOURDIEU, P. (1979),

"Les trois états du capital culturel", Actes de la recherche en sciences sociales, No. 30 ("L'institution scolaire"), S. 3-6.

BOURDIEU, P. (1980),

"Le capital social: notes provisoires", Actes de la recherche en sciences sociales I, No. 31, S. 2-3.

BOURDIEU, P. (1985),

"The Forms of Capital", in: J.E. Richardson (Hrsg.), Handbook of Theory of Research for the Sociology of Education, Greenwood Press, New York, S. 241-258.

BOURDIEU, P. und J.C. PASSERON (1970), Reproduction in Education, Society and Culture, Sage, London.

BRINK, S. und A. ZEESMAN (1997), "Measuring Social Well-Being: An Index of Social Health for Canada", HRDC, Working Paper, R-97-9E.

BROWN, G. und T. HARRIS (1978), Social Origins of Depression, Tavisock, London.

BRYK, A.S., V.E. LEE und P.B. HOLLAND (1993), Catholic Schools and the Common Good, Harvard University Press, Cambridge, MA.

BURT, R.S. (1992), Structural Holes, The Social Structure of Competition, Harvard University Press, Cambridge, MA.

BYNNER, J., S. MCINTOSH, A. VIGNOLES, L. DEARDEN, H. REED und J. VAN REENEN (2001), Wider Benefits of Learning Improving Adult Basic Skills: Benefits to the Individual and to Society, Bericht an das Department for Education and Employment (UK), DfEE Wider Benefits of Learning Research Centre, Institute of Education, London University, Centre for Economic Performance, London School of Economics und Institute for Fiscal Studies.

BYNNER, J. und M. EGERTON (2001),

The Wider Benefits of Higher Education, Bericht unter der Federführung von Higher Education Funding Council for England in Zusammenarbeit mit dem Smith Institute, Department for Education and Employment (UK), DfEE Wider Benefits of Learning Research Centre, Institute of Education, London University, Juli.

CAPPELLI, P. und N. ROGOVSKI (1994),

"New Work Systems and Skills Requirements", International Labour Review, No. 2, S. 205-220.

CARD, D. (1994),

"Earnings, Schooling, and Ability Revisited", NBER, Working Paper, No. 4832, August.

CARD, D. (1999),

"Causal Effect of Education on Earnings", in: O. Ashenfelter und D. Card (Hrsg.), Handbook of Labor Economics, Vol. 3A, Chapter 30, North-Holland, Amsterdam, S. 1801-1863.

CARLINER, G. (1996),

“The Wages and Language Skills of U.S. Immigrants”, NBER, Working Paper, No. 5793, Cambridge, MA.

CARLSON, M. (1999),

"Do Fathers Really Matter? Father Involvement and Socio-psychological Outcomes for Adolescents", BendheimThoman Center for Research on Child Wellbeing, Working Paper, 99-104, Princeton University.

COBB, C., T. HALSTEAD und J. ROWE (1996),

"If the GDP is Up, Why is America Down?", The Atlantic Monthly, Oktober, S. 59-78.

COHEN, P., E. STRUENING, G. MUHLIN, E. GENEVIE, S. KAPLAN und H. PECK (1982),

"Community Stressors, Mediating Conditions and Well-being in Urban Neighborhoods", Journal of Community Psychology, No. 10.

COLEMAN, J. (1988),

"Social Capital in the Creation of Human Capital", American Journal of Sociology, Vol. 94, Supplement, S. 95-120.

COLEMAN, J.S. (1990),

The Foundations of Social Theory, Harvard University Press, Cambridge.

COLEMAN, J. und T. HOFFER (1987),

Public and Private High Schools: The Impact of Communities, Basic Books, New York, S. 94, 133-135, 231, 229 (wegen entgegengesetzter Belege vgl. Stephen L. Morgan und Aage B. Sørensen, 1999, “A Test of Coleman’s Social Capital Explanation of School Effects”, American Sociological Review, No. 64, S. 661-681). 
COLEMAN, J., E. CAMPBELL, C. HOBSON, J. MCPARTLAND, A. MOOD, F. WEINFALL und R. YORK (1966), Equality of Educational Opportunity, Government Printing Office, Washington, U.S.

COLLIER, P. (1998),

"Social Capital and Poverty", Working Paper, Weltbank, Washington, D.C.

COUNCIL OF ECONOMIC ADVISERS (1999),

"Families and the Labor Market, 1969-1999: Analyzing the time crunch"”, ein Bericht der Wirtschaftsberater des Präsidenten, Washington, D.C., Mai.

COX, E. (2000),

in: R. Putnam (Hrsg.), Gesellschaft und Gemeinsinn, Bertelsmann-Stiftung.

COX, E. und D. MACDONALD (2000),

"Making Social Capital a Discussion Paper", New South Wales Council of Social Service.

DARLING, N. und L. STEINBERG (1997),

"Community Influences on Adolescent Achievement and Deviance", in: Brooks-Gunn, Duncan und Aber (Hrsg.), Neighborhood Poverty, Vol. II, S. 120-131.

De HART, J. und P. DEKKER (1999),

"Civic Engagement and Volunteering in the Netherlands: A Putnamian' Analysis", in: J. Van Deth, M. Maraffi, K. Newton und P. Whiteley (Hrsg.), Social Capital and European Democracy, Routledge, London, S. 75-107.

DE LA FUENTE, A. und R. DOMENECH (2000),

"Human Capital in Growth Regressions: How Much Difference does Data Quality Make?", CSIC, Campus de la Universidad Autonoma de Barcelona.

DOBELL, R. (2001),

"Social Capital and Social Learning in a Full World", in: J.F. Helliwell (Hrsg.), The Contribution of Human and Social Capital to Sustained Economic Growth and Well-being: International Symposium Report, Human Resources Development Canada und OECD.

DOUGHERTY, C. und D.W. JORGENSEN (1996),

"International Comparisons of the Sources of Growth", American Economic Review, Mai, S. 25-29.

DRUCKER, P. (1993),

The Post Capitalist Society, Butterworth-Heinemann, Oxford.

DURKHEIM, E. (1893),

The Division of Labor in Society, The Free Press, New York, 1984.

DURKHEIM, E. (1970),

Suicide: A Study of Sociology, Routledge \& Kegan Paul Ltd, London.

EASTERLY, W. und R. LEVINE (1997),

"Africa's Growth Tragedy: Policies and Ethnic Divisions", Quarterly Journal of Economics, Vol. 112(4), November, S. 1203-1250.

ECKERSLEY, R. (1998),

Measuring Progress, Is Life Getting Better?, Commonwealth Scientific and Industrial Research Organisation Publishing, Victoria, Australien.

ELIASSON, G. (2001),

"The Role of Knowledge in Economic Growth", in: J.F. Helliwell (Hrsg.), The Contribution of Human and Social Capital to Sustained Economic Growth and Well-being: International Symposium Report, Human Resources Development Canada und OECD.

ENGLANDER, A.S. und A. GURNEY (1994),

“Medium-term Determinants of OECD Productivity”, OECD, Economic Studies, No. 22, OECD, Paris.

EPSTEIN, J.L. (1995),

"School/Family/Community Partnerships: Caring for the Children we Share", Phi Delta Kappan, Vol. 76, S. 701-712.

ERIKSON, R. und J. JONSSON (1996),

"Explaining Class Inequality in Education: the Swedish Test Case", in: R. Erikson und J.O. Jonsson (Hrsg.), Can Education Be Equalized?, Westview Press, Boulder, CO.

(C) OECD 2004 
FIELD, J. und L. SPENCE (2000),

"Informal learning and social capital", in: F. Coffield (Hrsg.), The Necessity of Informal Learning, Policy Press, Bristol.

FRATIGLIONI, L., H. WANG, K. ERICSSON, M. MAYTAN und B. WINBLAD (2000),

"Influence of Social Network on Occurrence of Dementia: A Community-based Longitudinal Study", The Lancet, Vol. 355, No. 9212, 15. April.

FREEMAN, R.B., M.M. KLEINER und C. OSTROGOFF (1997),

“The Anatomy and Effects of Employee Involvement”, Vorlage für die Tagung der American Economic Association.

FULLER, B. und P. CLARKE (1994),

"Raising School Effects while Ignoring Culture? Local Conditions and the Influence of Classroom Tools, Rules and Pedagogy", Review of Educational Research, Frühling, Vol. 64, No. 1, S. 119-157.

FULLER, B. und S. HEYNEMAN (1989),

“Third World School Quality. Current Collapse, Future Potential”, Educational Researcher, Vol. 18(2), S. 12-19.

FUKUYAMA, F. (1995),

Trust: The Social Virtues and the Creation of Prosperity, The Free Press, New York.

FUKUYAMA, F. (1999),

The Great Disruption: Human Nature and the Reconstitution of Social Order, The Free Press, New York.

GALLAND, O. (1999),

"Les Relations de Confiance", La Revue Tocqueville, The Tocqueville Review, Vol. XX, No.1.

GALLIE, D., J. GERSHUNY und C. VOGLER (1994),

"Unemployment, the Household and Social Networks", in: Gallie et al. (Hrsg.), Social Change and the Experience of Unemployment, Oxford University Press.

GARBARINO, J. und D. SHERMAN (1980),

"High-Risk Neighborhoods and High-Risk Families: The Human Ecology of Child Maltreatment", Child Development, No. 51, S. 188-198.

GASKIN, K. und J.D. DAVIS SMITH (1995),

A New Civic Europe? A Study of the Extent and Role of Volunteering, The Volunteer Center, London.

GEERTZ, C. (1962),

Social Change and Economic Modernization in Two Indonesian Towns : A Case in Point, Bobbs-Merrill, Indianapolis.

GEMMELL, N. (1995),

"Endogenous Growth, the Solow Model and Human Capital", Economics of Planning, No. 28, S. 169-183.

GEMMELL, N. (1996),

"Evaluating the Impacts of Human Capital Stocks and Accumulation on Economic Growth: Some New Evidence", Oxford Bulletin of Economics and Statistics, No. 58, S. 9-28.

GEMMELL, N. (1997),

"Externalities to Higher Education: A Review of the New Growth Literature", Bericht 8 in National Committee of Inquiry into Higher Education, Higher Education in the Learning Society, Berichte 5-9, S. 109-149.

GINGRAS, Y. und R. ROY (1998),

"Is There a Skill Gap in Canada?", Applied Research Working Paper Series, Human Resources Development Canada.

GITTLEMAN, M. und E.N. WOLFF (1995),

"R\&D Activity and Cross-country Growth Comparisons", Cambridge Journal of Economics, Vol. 19, S. 189-207.

GLAESER, E.L. (2001),

"The Formation of Social Capital", in: J.F. Helliwell (Hrsg.), The Contribution of Human and Social Capital to Sustained Economic Growth and Well-being: International Symposium Report, Human Resources Development Canada und OECD.

GRANOVETTER, M. (1973),

"The Strength of Weak Ties", American Journal of Sociology, No. 78, S. 1360-1380.

GREEN, F., D. ASHTON, B. BURCHELL, B. DAVIES und A. FELSTEAD (1997),

"An Analysis of Changing Work Skills in Britain", Vorlage für die "Analysis of Low Wage Employment Conference", Centre for Economic Performance, London School of Economics, 12.-13. Dezember. 
GREEN, F., S. MCINTOSH und A. VIGNOLES (1999),

"Overeducation and Skills - Clarifying the Concepts", Centre for Economic Performance, Labour Market Programme Discussion Paper, No. 435, London School of Economics.

GRILICHES, Z. (1996),

"Education, Human Capital, and Growth: A Personal Perspective", NBER, Working Paper, No. 5426, Januar.

GROGGER, J. (1996),

"Does School Quality Explain the Recent Black/White Wage Trend?", Journal of Labor Economics, Vol. 14(2), April, S. 231-253.

GUISO, L., P. SAPIENZA und L. ZINGALES (2000),

"The Role of Social Capital in Financial Development", NBER, Working Paper, No. 7563, Februar.

GUNDLACH, E., L. WOSSMANN und J. GMELIN (2000),

"The Decline of Schooling Productivity in OECD Countries", Vorlage für die Jahrestagung der Royal Economic Society, St Andrews, 10.-13. Juli.

HALL, P. (1999),

"Social Capital in Britain", British Journal of Political Science, No. 29, S. 417-461.

HALL, R. und C. JONES (1999),

"Why Do Some Countries Produce So Much More Output per Worker than Others?", Quarterly Journal of Economics, Februar, Vol. 114, S. 83-116.

HALPERN, D.S. (2001),

“Moral Values, Social Trust and Inequality: Can Values Explain Crime?", British Journal of Criminology, Vol. 41(2).

HALPERN, D.S. und J. NAZROO (2000),

"The Ethnic Density Effect: Results from a National Community Survey of England and Wales", International Journal of Social Psychiatry, Vol. 46 (1), S. 34-46.

HANIFAN, L. (1916),

"The Rural School Community Center", Annals of the American Academy of Political and Social Science, No. 67.

HANUSHEK, E.A. (1992),

“The Trade-Off between Child Quantity and Quality", Journal of Political Economy 100, No. 1, Februar, S. 84-117.

HANUSHEK, E.A. (1998),

"The Evidence on Class Size", Occasional Paper, No. 98-101, W. Allen Wallis Institute of Political Economy, University of Rochester, Februar.

HANUSHEK, E.A. und D. KIM (1995),

"Schooling, Labor Force Quality, and Economic Growth", NBER, Working Paper, No. 5399, Dezember.

HANUSHEK, E.A. und D.D. KIMKO (2000),

"Schooling, Labor Force Quality, and the Growth of Nations", The American Economic Review, Vol. 90, No. 5, Dezember.

HANUSHEK, E.A. und J. SOMERS (1999),

"Schooling, Inequality and the Impact of Government", Vorlage für die Konferenz "Increasing Income Inequality in America", Texas A\&M University, März.

HANUSHEK, E.A., J. KAIN und S. RIVKIN (1998),

"Teachers, Schools, and Academic Achievement”, NBER, Working Paper, No. 6691, August.

HANUSHEK, E.A., J. KAIN und S. RIVKIN (1999),

“Do Higher Salaries Buy Better Teachers?”, NBER, Working Paper, No. 7802, März.

HANUSHEK, E.A., S. RIVKIN und L. TAYLOR (1996),

"Aggregation and the Estimated Effects of School Resources", Review of Economics and Statistics, November, Vol. 78(4), S. 611-627.

HANUSHEK, E., J. KAIN, J. MARKMAN und S. RIVKIN (2000),

"Do Peers Affect Student Achievement?", Vorlage für die Konferenz "Empirics of Social Interactions", Brookings Institution, 14.-15. Januar.

HAO, L. (1994),

Kin Support, Welfare, and Out-of-Wedlock Mothers, Garland, New York.

(C) OECD 2004 
HARBERGER, A. (1998),

“A Vision of the Growth Process", American Economic Review, Vol. 88, No. 1, März.

HARMON, F. und I. WALKER (1995),

"Estimates of the Economic Return to Schooling for the United Kingdom", American Economic Review, No. 85, S. 1278-1286.

HARTOG, J. (1997),

"On Returns to Education: Wandering along the Hills of ORU Land", Leitreferat anlässlich der LVII. Konferenz “Applied Econometrical Association”, Maastricht, Mai.

HAVEMAN, R H. und B. WOLFE (1984),

"Schooling and Economic Well-Being: The Role of Non-market Effects", Journal of Human Resources, No. 19, Sommer, S. 378-407.

HECKMAN, J. (1999),

"Policies to Foster Human Capital”, NBER, Working Paper, No. 7288, Cambridge, MA, August.

HELLIWELL, J.F. (1996),

"Economic Growth and Social Capital in Asia", NBER, Working Paper, No. 5470, Cambridge, MA.

HELLIWELL, J.F. (2001),

"The Contribution of Human and Social Capital to Sustained Economic Growth", The Contribution of Human and Social Capital to Sustained Economic Growth and Well-being: International Symposium Report, Human Resources Development Canada und OECD.

HELLIWELL, J.F. und R. PUTNAM (1999a),

"Economic Growth and Social Capital in Italy", in: P. Dasgupta und I. Serageldin (Hrsg.), Social Capital: a Multifaceted Perspective, Weltbank.

HELLIWELL, J.F. und R. PUTNAM (1999b),

"Education and Social Capital", NBER, Working Paper, No. 7121, Cambridge, MA

HENDERSON, A. und N. BERLA (1994),

A New Generation of Evidence: The Family is Critical to Student Achievement, National Committee for Citizens in Education, Washington, D.C.

HERS, J. (1998),

"Human Capital and Economic Growth", GPB Report, Vol. 98(2).

HESTON, A. und R. SUMMERS (1988),

"A New Set of International Comparisons of Real Product and Price Levels Estimates for 130 Countries, 1950-1985", Review of Income and Wealth, XXXIV, S. 1-26.

HESTON, A. und R. SUMMERS (1991),

"The Penn World Table (Mark 5): An Expanded Set of International Comparisons, 1950-1988", Quarterly Journal of Economics, Mai, S. 327-368.

HIRSCHMAN, A. (1984),

"Against Parsimony: Three Easy Ways of Complicating Economic Analysis", American Economic Review, No. 74, S. 88-96.

HJERRPE, R. (1998),

"Social Capital and Economic Growth", Discussion Paper, No. 183, Government Institute for Economic Research (VATT), November.

HO SUI-CHU, E. und J.D. WILLMS (1996),

"The Effects of Parental Involvement on Eighth Grade Achievement", Sociology of Education, No. 69, S. 126-141.

HODGKINSON, V. und M. WEITZMAN (1988),

Giving and Volunteering in the United States: Findings from a National Survey, Independent Sector, Washington, D.C.

HOXBY, C. (1994),

"Do Private Schools Provide Competition for Public Schools?", NBER, Working Paper, No. 4978, New York, Dezember.

HUMAN RESOURCES DEVELOPMENT CANADA (1999),

"The Social Context of Productivity: Challenges for Policy Makers", Aufzeichnungen für einen Vortrag von J. Lahey vor dem "Queen's International Institute on Social Policy", 25. August. 
HUMPHREY, J. und H. SCHMITZ (1998),

"Trust and Inter-firm Relations in Developing and Transition Economies", The Journal of Development Studies, 34(4), S. 32-45.

INGLEHART, R. (1997),

Modernization and Postmodernization: Cultural, Economic and Political Change in 43 Societies, Princeton University Press, Princeton.

INGLEHART, R. (2000),

"Globalization and Postmodern Values", The Washington Quarterly, Winter.

INOGUCHI, T. (2000),

in: R. Putnam (2000b), Gesellschaft und Gemeinsinn, Bertelsmann-Stiftung.

JACOBS, J. (1961),

The Life and Death of Great American Cities, Random House, New York.

JENKINS, H. (1995a),

Education and Production in the United Kingdom, Economics Discussion Paper, No. 101, Nuffield College, Oxford.

JENKINS, H. (1995b),

Infrastructure, Education and Productivity: A Multi-Country Study (Doktorarbeit), Nuffield College, Oxford Universität.

JENSON, J. (1998),

"Mapping Social Cohesion: The State of Canadian Research", Canadian Policy Research Networks Study.

JONES, C.I. (1996),

"Human Capital, Ideas and Economic Growth", Vorlage für das VIII. Internationale Wirtschaftsseminar "Villa Mondragone" über Finanzwesen, Forschung, Bildung und Wachstum, Rom, Italien, Juni.

JORGENSON, D. und B. FRAUMENI (1987),

"The Accumulation of Human and Non-human Wealth, 1948-1984", unveröffentlichtes Manuskript, Harvard Universität.

JOSHI, H., E. COOKSEY, R. WIGGINS, A. MC CULLOCH, G. VERROPOULOU und L. CLARKE (1999), "Diverse Family Living Situations and Child Development: A Multi-level Analysis Comparing Longitudinal Evidence from Britain and the United States", International Journal of Law, Policy and the Family, No. 13, S. 292-314.

KAWACHI, I., B.P. KENNEDY, K. LOCHNER und D. PROTHROW-STITH (1997), "Social Capital, Income Inequality, and Mortality", American Journal of Public Health, Vol. 87 (9), S. 1491-1499.

KEESE, M. und A. PUYMOYEN (2001),

"Changes in Earnings Structure: Some International Comparisons Using the OECD Structure of Earnings Database", OECD, Labour Market and Social Policy Occasional Papers, Paris.

KELLAGHAN, T. (1999),

"Educational Disadvantage: An Analysis", Vorlage für die "Irish Department of Education and Science Conference of Inspectors", Killarney, 6.-8. Dezember.

KELLAGHAN, T., K. SLOANE, B. ALVAREZ und B. BLOOM (1993),

The Home Environment and School Learning. Promoting Parental Involvement in the Education of Children, JosseyBass, San Francisco.

KENDRICK, J. (1976),

The Formation and Stocks of Total Capital, Columbia University Press für NBER, New York.

KENKEL, D. (1991),

"Health Behavior, Health Knowledge, and Schooling", Journal of Political Economy, Vol. 99(2), S. 287-305.

KENNEDY, I.B.P., K. LOCHNER und D. PROTHROW-STITH (1997),

"Social Capital, Income Inequality, and Mortality", American Journal of Public Health, Vol. 87, No. 1.

KERN, H. (1998),

"Lack of Trust, Surfeit of Trust: Some Causes of the Innovation Crisis in German Industry", in: C. Land und R. Bachmann (Hrsg.), Trust within and between Organizations, Oxford University Press, New York, S. 203-213.

KING, R. und R. LEVINE (1994),

"Capital Fundamentalism, Economic Development and Economic Growth", Carnegie-Rochester Series on Public Policy, No. 40. 
KORBIN, J. und C. COULTON (1997),

"Understanding the Neighborhood Context for Children and Families: Combining Epidemiological and Ethnographic Approaches", in: J. Brooks-Gunn, G.J. Duncan und J.L. Aber (Hrsg.), Neighborhood Poverty, Vol. II, Russell Sage Foundation, New York, S. 65-79.

KNACK, S. (1999),

"Social Capital, Growth and Poverty; A Survey of Cross-Country Evidence", Social Capital Initiative, Working Paper, No. 7, Weltbank.

KNACK, S. (2001),

"Trust, Associational Life and Economic Performance", in: J.F. Helliwell (Hrsg.), The Contribution of Human and Social Capital to Sustained Economic Growth and Well-being: International Symposium Report, Human Resources Development Canada und OECD.

KNACK, S. und P. KEEFER (1997),

"Does Social Capital Have an Economic Payoff? A Cross-Country Investigation", Quarterly Journal of Economics, Vol. 112(4), S. 1251-1288.

KRUEGER, A. und M. LINDAHL (1999),

"Education for Growth in Sweden and the World", NBER, Working Paper, No. 7190.

KUZNETS, S. (1962), in: The New Republic, Washington D.C, 20. Oktober.

KYRIACOU, G. (1991), Level and Growth Effects of Human Capital: A Cross-Country Study of the Convergence Hypothesis, C.V. Starr Centre, Working Paper, No. 91-26, New York.

LA PORTA, R., F. LOPEZ-DE-SILANES, A. SHLEIFER und R.W. VISHNY (1997),

“Trust in Large Organisations”, American Economic Review, Papers and Proceedings, Vol. 87(2), S. 333-338.

LANDSBURG, S. (1993),

The Armchair Economist: Economics and Everyday Life, The Free Press, New York.

LEANA, C.R. und H.J. van BUREN (1999),

"Organizational Social Capital and Employment Practices", Academy of Management Review, Vol. 24, No. 3, S. 538-555.

LEE, J.W. und R.J. BARRO (1997),

"Schooling Quality in a Cross-Section of Countries", NBER, Working Paper, No. 6198.

LESSER, E.L. (2000),

Knowledge and Social Capital, Butterworth-Heinemann, Boston.

LEVY, F. und R.J. MURNANE (1999),

"Are there Key Competencies Critical to Economic Success? An Economics Perspective", Vorlage für das OECDSymposium "Definition and Selection of Competencies", Oktober.

LOURY, G. (1987),

"Why Should we Care about Group Inequality?", Social Philosophy and Policy, S. 249-271.

LUCAS, R.E. (1988),

"On the Mechanisms of Economic Development", Journal of Monetary Economics, Vol. 22.

LUNDVALL, B.-A. und B. JOHNSON (1994),

"The Learning Economy", Journal of Industry Studies, Vol. 1, No. 2, S. 23-42.

LUNDVALL, B.-A. und P. MASKELL (1999),

"Nation States and Economic Development: From National Systems of Production to National Systems of Knowledge Creation and Learning", in: G.L. Clark et al. (Hrsg.), Handbook of Economic Geography, Kapitel 10, Oxford University Press, London.

LYNCH, J., P. DUE, C. MUNTANER und G. DAVEY SMITH (2001),

"Social Capital - Is it a Good Investment Strategy for Public Health", Journal of Epidemiology and Community Health, Vol. 54, S. 404-408.

MADDISON, A. (1987),

"Growth and Slowdown in Advanced Capitalist Economies: Techniques of Quantitative Assessment", Journal of Economic Literature, No. 25, S. 649-698. 
MADDISON, A. (1991),

Dynamic Forces in Capitalist Development, Oxford University Press, Oxford.

MANKIW, N.G. (1995),

"The Growth of Nations", Brookings Papers on Economic Activity, No. 1, S. 275-326.

MANKIW, N.G., D. ROMER und D.N. WEIL (1992),

"A Contribution to the Empirics of Economic Growth", Quarterly Journal of Economics, No. 107, S. 407-437.

McLANAHAN, S. und G.D. SANDEFUR (1994),

Growing Up with a Single Parent: What Hurts, What Helps, Harvard University Press, Cambridge, MA.

McMAHON, W.W. (2001),

"The Impact of Human Capital on Non-Market Outcomes and Feedbacks on Economic Development in OECD Countries", in: J.F. Helliwell (Hrsg.), The Contribution of Human and Social Capital to Sustained Economic Growth and Well-being: International Symposium Report, Human Resources Development Canada und OECD.

MINCER, J. (1974),

"Schooling, Experience and Earnings", NBER, Cambridge, MA.

MINGAT, A. und J. TAN (1996),

"The Full Social Returns to Education: Estimates Based on Countries' Economic Growth Performance", Human Capital Development Papers, Weltbank, Washington, D.C.

MOORE, G. (1990),

"Structural Determinants of Men's and Women's Personal Networks", American Sociological Review, Vol. 55, Oktober.

MOSTELLER, F. (1995),

"The Tennessee Study of Class Size in the Early School Grades", The Future of Children 5, No. 2, Sommer/Herbst, S. 113-127.

MUNTANER, C., J. LYNCH und G.D. SMITH (2000),

"Social Capital and the Third Way in Public Health", Critical public Health, Vol. 10, No. 2.

MURNANE, R.J. (1975),

Impact of School Resources on the Learning of Inner City Children, Ballinger, Cambridge, MA.

MYERS, D. (1999),

"Close Relationships and Quality of Life", in: D. Kahneman, E. Diener und E. Schwartz (Hrsg.), Well-being: The Foundations of Hedonic Psychology, Russell Sage Foundation, New York.

NARAYAN, D. und L. PRITCHETT (1998),

"Cents and Sociability: Household Income and Social Capital in Rural Tanzania", Economic Development and Cultural Change, Weltbank, Washington.

NEHRU, V. und A. DHARESHWAR (1994),

"New Estimates of Total Factor Productivity Growth for Developing and Industrial Countries", Policy Research Working Paper, 1313, Weltbank, Washington D.C.

NEHRU, V., E. SWANSON und A. DUBEY (1995),

"A New Database on Human Capital Stock in Developing and Industrial Countries: Sources, Methodology and Results", Journal of Development Economics, No. 46, S. 379-401.

NIE, N.H., J. JUNN und K. STEHLIK-BARRY (1996),

Education and Democratic Citizenship in America, University of Chicago Press, Chicago.

NONNEMAN, W. und P. VANHOUDT (1996),

"A Further Augmentation of the Solow Model and the Empirics of Economic Growth for OECD Countries", Quarterly Journal of Economics, No. 111, S. 943-953.

NORDHAUS, W. und J. TOBIN (1972),

"Is growth obsolete?", in: Economic Growth, Fiftieth Anniversary Colloquium, Vol. 5, NBER, New York.

NORTH, D. (1990),

Institutions, Institutional Change and Economic Performance, Cambridge University Press, New York.

NORTON, A. (1998),

“The Welfare State: Depreciating Australia's Social Capital?", Policy, S. 41-43.

(C) OECD 2004 
NYE, B. und L. HEDGES (2000),

"The Effects of Small Classes on Academic Achievement: The Results of the Tennessee Class Size Experiment", American Educational Research Journal, Vol. 37, No. 1, Frühling, S. 123-151.

OECD (1976),

Measuring Social Well-being: A Progress Report on the Development of Social Indicators, Paris.

OECD (1996),

Reconciling Economy and Society: Towards a Plural Economy, Territorial Development Service, Paris.

OECD (1998),

Human Capital Investment - An International Comparison, Paris.

OECD (1999a),

Bildungspolitische Analyse, Paris.

OECD (1999b),

Interim Report on the OECD Three-Year Project on Sustainable Development, Paris.

OECD (2000a)

Knowledge Management in the Learning Society, Paris.

OECD (2000b),

"Links between Policy and Growth: Cross-country evidence", Entwurf eines Papiers der Arbeitsgruppe 1 der Hauptabteilung Wirtschaft.

OECD (2001),

Bildungspolitische Analyse, Paris.

OECD und Statistics Canada (2000),

Literacy in the Information Age: Final Report of the International Adult Literacy Survey, Paris.

OECD und US Department of Education (1998),

How Adults Learn, Tagungsbericht einer Konferenz unter der Schirmherrschaft des US-Bildungsministeriums, Washington D.C., April.

OFFE, C. und S. FUCHS (1998),

"A Decline of Social Capital? - The German Case", in: R. Putnam (2000b), Gesellschaft und Gemeinsinn, Bertelsmann-Stiftung.

OHSAKO, T. (1998),

"Learning and Social Participation by Senior Citizens in Japan: Analysis of Major Issues from an International Perspective", in: How Adults Learn, Tagungsbericht einer Konferenz unter der Schirmherrschaft der OECD und des US-Bildungsministeriums, Washington D.C., April.

OLSON, M. (1982),

The Rise and Decline of Nations, Yale University Press, New Haven.

OMORI, T. (2001),

"Balancing Economic Growth with Well-being: Implication of the Japanese Experience", in: J.F. Helliwell (Hrsg.), The Contribution of Human and Social Capital to Sustained Economic Growth and Well-being: International Symposium Report, Human Resources Development Canada und OECD.

OSBERG, L. (1985),

"The Measurement of Economic Welfare," in: D. Laidler (Koordinator), Approaches to Economic Well-being, Vol. 26 des Research Program of the Royal Commission of the Economic Union und Development Prospects for Canada (MacDonald Commission), University of Toronto Press, Toronto.

OSBERG, L. (1992),

"Sustainable Social Development", in: R.C. Allen und G. Rosenbluth (Hrsg.), False Promises: The Failure of Conservative Economics, New Star Books, Vancouver, S. 227-242.

OSBERG, L. (2001),

"Comparisons of Trends in GDP and Economic Well-being - The Impact of Social Capital", in: J.F. Helliwell (Hrsg.), The Contribution of Human and Social Capital to Sustained Economic Growth and Well-being: International Symposium Report, Human Resources Development Canada und OECD. 
PAINTER, G. und D. LEVINE (1999),

"Family Structure and Youths' Outcomes: Which Correlations are Causal?", Institute of Industrial Relations, Working Paper Series, No. 69, September.

PEARCE, D. und G. ATKINSON (1997),

"The Concept of Sustainable Development: An Evaluation of its Usefulness Ten Years after Bruntland", Centre for Social and Economic Research on the Global Environment, University College London and University of East Anglia.

PICCIOTTO, R. (1998),

"Gender and Social Capital", Referat anlässlich des Seminars "Gender and Development”, Weltbank, April (www.worldbank.org/html/prmge/know/gendev/).

PORTER, M. (1990),

The Competitive Advantage of Nations, The Free Press, New York.

PORTES, A. (1998),

"Social Capital: Its Origins and Applications in Modern Sociology", Annual Review of Sociology, No. 24, S. 1-24.

PORTES, A. und P. LANDOLT (1996),

"The Downside to Social Capital”, The American Prospect, No. 26, 94, Mai-Juni, S. 18-21.

PORTES, A. und J. SENSENBRENNER (1993),

"Embeddedness and Immigration: Notes on the Social Determinants of Economic Action", American Journal of Sociology, 98(6), S. 1320-1350.

POWELL, D.R. (1989),

"Families and Early Childhood Progams", National Association for the Education of Young Children, Washington D.C.

POWELL, W. und L. SMITH-DOERR (1994),

"Networks and Economic Life", in: N.J. Smelser und R. Swedberg (Hrsg.), The Handbook of Economic Sociology, Prince-ton University Press, Princeton, S. 368-402.

PRITCHETT, L. (1999),

Where Has All the Education Gone?, Weltbank, Washington D.C.

PSACHAROPOULOS , G. (1994),

"Returns to Investment in Education: A Global Update", World Development, Vol. 22(9), S. 1325-1343.

PUTNAM, R. (1993),

Making Democracy Work, Princeton University Press, Princeton.

PUTNAM, R.D. (1995),

"Bowling Alone: America's Declining Social Capital”, Journal of Democracy, Vol. 6, No. 1, S. 65-78 (muse.jhu.edu/ demo/journal_of_democracy/v006/putnam.html).

PUTNAM, R. (2000a),

Bowling Alone: The Collapse and Revival of American Community, Simon Schuster, New York.

PUTNAM, R. (2000b),

Gesellschaft und Gemeinsinn, Bertelsmann-Stiftung.

PUTNAM, R. (2001),

"Social Capital: Measurement and Consequences", in: J.F. Helliwell (Hrsg.), The Contribution of Human and Social Capital to Sustained Economic Growth and Well-being: International Symposium Report, Human Resources Development Canada und OECD.

RAUDENBUSH, S.W. und R.M. KASIM (1998),

"Cognitive Skill and Economic Inequality: Findings from the National Adult Literacy Survey", Harvard Educational Review, Vol. 68 (1), S. 33-79.

RITZEN, J. (2001)

"Social Cohesion, Public Policy, and Economic Growth: Implications for OECD Countries", in: J.F. Helliwell (Hrsg.), The Contribution of Human and Social Capital to Sustained Economic Growth and Well-being: International Symposium Report, Human Resources Development Canada und OECD.

RIVERA-BATIZ, F.L. (1994),

"Quantitative Literacy and the Likelihood of Employment among Young Adults in the United States", Journal of Human Resources, Vol. XXVII, No. 2, S. 313-328.

(C) OECD 2004 
RIVKIN, S.G., E.A. HANUSHEK und J.F. KAIN (1998),

"Teachers, Schools, and Academic Achievement", Vorlage für die Jahrestagung der Econometric Society, Chicago, Januar.

RIZZO, J. und R. ZECKHAUSER (1992),

"Advertising and the Price, Quantity, and Quality of Primary Care Physician Services", Journal of Human Resources, 27(3), S. 381-421.

RODRIK , D. (1998),

"Where Did All the Growth Go? External Shocks, Social Conflict and Growth Collapses", NBER, Working Paper, No. 6350.

RODRIK, D. (2000),

"Development Strategies for the Next Century", Vorlage für die Konferenz "Developing Economies in the 21st Century", Institute for Developing Economies, Japan External Trade Organization, Januar, 2000 (www.ksg.harvard. edu/rodrik/devstrat.PDF).

ROMER, P.M. (1990a),

"Human Capital and Growth: Theory and Evidence", Carnegie-Rochester Conference Series on Public Policy, No. 32, S. 251-286.

ROMER, P.M. (1990b), “Endogenous Technological Change”, Journal of Political Economy, 98(5), Part 2, S. 71-102.

ROSE, R. (2000),

"How Much Does Social Capital Add to Individual Health? A Survey Study of Russians", Social Science and Medicine, I-15 Pergamon.

ROTHSTEIN, B. (1998),

"Social Capital in the Social Democratic State - The Swedish Model and Civil Society", Department of Political Science, Universität Göteborg, Schweden.

ROUSE, C.E. (1998),

"Schools and Student Achievement: More Evidence from the Milwaukee Parental Choice Program", Economic Policy Review, März, 4(1), S. 61-78.

RUNYAN, D., W. HUNTER et al. (1998),

"Children Who Prosper in Unfavorable Environments: The Relationship to Social Capital", Pediatrics, 101, S. 12-18.

SALA-I-MARTIN, X. (1997),

"I Just Ran Four Million Regressions", NBER, Working Paper, No. 6252.

SAMPSON, R. (1995),

"The Community", in: J. Wilson und J. Petersilia (Hrsg.), Crime, Institute For Contemporary Studies Press, San Francisco, S. 193-216.

SAMPSON, R. und J. MORENOFF (1997),

"Ecological Perspectives on the Neighborhood Context of Urban Poverty: Past and Present", in: J. Brooks-Gunn, G. Duncan und J.L. Aber (Hrsg.), Neighborhood Poverty: Volume II, Russell Sage Foundation, New York, S. 1-22.

SAMPSON, R., S. RAUDENBUSH und F. EARLS (1997), "Crime: A Multilevel Study of Collective Efficacy", Science, 277, 15. August.

SANDEFUR, G.D., S. MCLANAHAN und R.A. WOJTKIEWICZ (1989), "Race and Ethnicity, Family Structure, and High School Graduation", Discussion Paper, No. 893-889, Institute for Research on Poverty.

SCARPETTA, S., A. BASSANINI, D. PILAT und P. SCHREYER (2000),

"Economic Growth in the OECD Area: Recent Trends at the Aggregate and Sectoral Level", OECD, Economics Department Working Papers, No. 248.

SCHULLER, T., J. BYNNER, A. GREEN, L. BLACKWELL, C. HAMMOND und J. PRESTON (2001), "Modelling and Measuring the Wider Benefits of Learning: An Initial Synthesis", Centre for Research on the Wider Benefits of Learning Institute of Education/Birkbeck College.

SEN, A. (1987), The Standard of Living, Cambridge University Press, Cambridge. 
SHIMADA, H. (1988),

"Hyu-man Uea no Keizaigaku (Economics of Humanware)", in: Omori (2001), Iwanami.

SHLEIFER, A. (1998),

"State Versus Private Ownership", Journal of Economics Perspectives, 12 (4), S. 133-150.

SIMONS, R. (1996),

Understanding Differences between Divorced and Intact Families: Stress, Interaction and Child Outcome, Thousand

Oaks, CA, Sage.

SMITH, A. (1776),

Der Wohlstand der Nationen, Band I.

SOLOW, R.M. (1956),

"A Contribution to the Theory of Economic Growth", Quarterly Journal of Economics, 70, S. 65-94.

SPENCE , A. (1973),

"Job Market Signalling”, Quarterly Journal of Economics 87, S. 355-374.

STEEDMAN, H. (1996),

"Measuring the Quality of Educational Outputs: A Note", Center for Economic Performance, Discussion Paper, No. 302, LSE.

TEACHMAN, J., K. PAASCH und K. CARVER (1999),

"Social Capital and the Generation of Human Capital", Social Forces, 75.

TEMPLE, J. (1999a),

“The New Growth Evidence”, Journal of Economic Literature, März, 37(1), S. 112-156.

TEMPLE , J. (1999b),

“A Positive Effect of Human Capital on Growth”, Economics Letters, 66, S. 131-134.

TEMPLE , J. (2001),

"Growth Effects of Education and Social Capital in the OECD", in: J.F. Helliwell (Hrsg.), The Contribution of Human and Social Capital to Sustained Economic Growth and Well-being: International Symposium Report, Human Resources Development Canada und OECD.

TEMPLE, J. und P. JOHNSON (1998),

"Social Capability and Economic Growth", Quarterly Journal of Economics, August, S. 965-988.

UNITED NATIONS DEVELOPMENT PROGRAMME (1990),

Human Development Report 1990, Oxford University Press, New York, NY.

UNITED NATIONS DEVELOPMENT PROGRAMME (2000),

Human Development Report 2000, Oxford University Press, New York, NY.

UZZI, B. (1996),

"The Sources and Consequences of Embeddedness for the Economic Performance of Organizations: The Network Effect", American Sociological Review, 61(4), S. 674-698.

UZZI, B. (1997),

"Social Structure and Competition in Inter-firm Networks: The Paradox of Embeddedness", Administrative Science Quarterly, 42(1), S. 35-67.

VAN ARK, B. und H. de JONG (1996),

"Accounting for Economic Growth in the Netherlands since 1913", Forschungsbericht der Universität Groningen, GD-26.

VERBA, S., K.L. SCHLOZMAN und H.E. BRADY (1995),

Voice and Equality: Civic Voluntarism in American Politics, Harvard University Press, Cambridge, M.A.

WEISS, A. (1995),

"Human capital vs. signalling explanations of wages", Journal of Economic Perspectives, 9(4), S. 133-154.

WELTBANK (1998),

"The Initiative on Defining, Monitoring and Measuring Social Capital Text of Proposals Approved for Funding", Environmentally and Socially Sustainable Development Network, Social Capital Initiative, Working Paper, No. 2, Juni.

(C) OECD 2004 
WHITE, M. und G. KAUFMAN (1997),

"Language Usage, Social Capital, and School Completion among Immigrants and Native-born Ethnic Groups", Social Science Quarterly, 78(2), S. 385-393.

WILKINSON, R. (1996),

Unhealthy Societies: The Afflictions of Inequality, Routledge, London.

WILLMS, J.D. (1999),

"Proposal for the Measurement of Socioeconomic Status for the Focused Component of the Student Questionnaire for the OECD Programme for International Student Assessment (OECD-PISA)", The Canadian Research Institute for Social Policy (CRISP), University of New Brunswick.

WILLMS, J. D. (2001),

"Three Hypotheses about Community Effects", in: J.F. Helliwell (Hrsg.), The Contribution of Human and Social Capital to Sustained Economic Growth and Well-being: International Symposium Report, Human Resources Development Canada und OECD.

WOLFE, B. und R. HAVEMAN (2001),

"Accounting for the Social and Non-market Benefits of Education", in: J.F. Helliwell (Hrsg.), The Contribution of Human and Social Capital to Sustained Economic Growth and Well-being: International Symposium Report, Human Resources Development Canada und OECD.

WOLFE, B.L. und S. ZUVEKAS (1997), Nonmarket Outcomes of Schooling, University of Wisconsin, Madison, Mimeo.

WOLFF, E.N. und M. GITTLEMAN (1993), "The Role of Education in Productivity Convergence: Does Higher Education Matter?", in: A. Szimai, B. Van Ark und D. Pilat (Hrsg.), Explaining Economic Growth, Elsevier Science Publishers.

WOOLCOCK, M. (1998),

"Social Capital and Economic Development: Toward a Theoretical Synthesis and Policy Framework", Theory and Society, 27(2), S. 151-208.

WOOLCOCK, M. (1999),

"Social Capital: The State of the Notion”, Vorlage für ein multidisziplinäres Seminar über Sozialkapital: "Global and Local Perspectives", Helsinki, 15. April.

WOOLCOCK, M. (2001),

"The Place of Social Capital in Understanding Social and Economic Outcomes", in: J.F. Helliwell (Hrsg.), The Contribution of Human and Social Capital to Sustained Economic Growth and Well-being: International Symposium Report, Human Resources Development Canada und OECD.

WORMS, J.P. (1998a),

"Old and New Civic and Social Ties in France", in: Putnam (2000b).

WORMS, J.P. (1998b),

"La crise du lien social, le problème du chaînon manquant", Empan, No. 32, Dezember, S. 94-104.

WORMS, J.P. (2000),

"Old and New Civic and Social Ties in France", in: R. Putnam (Hrsg.), Gesellschaft und Gemeinsinn, BertelsmannStiftung.

WOSSMANN, L. (2000),

"Schooling Resources, Educational Institutions and Student Performance: The International Evidence", Kiel, Working Paper, No. 983, Mai.

YANKELOVICH, D. (1981), New Rules: Searching for Self-fulfillment in a World Turned Upside Down, Bantam Books, New York. 
OECD PUBLICATIONS, 2, rue André-Pascal, 75775 PARIS CEDEX 16 PRINTED IN FRANCE

(96 2001015 P) ISBN 92-64-01553-1 - No. 534572004 